Marta Aparicio Arroyo

\title{
The Geometry of $\mathrm{SO}(p, q)$-Higgs Bundles
}

Departamento de Matemáticas

Facultad de Ciencias de la Universidad de Salamanca 2009 

Marta Aparicio Arroyo

\section{The Geometry of $\mathrm{SO}(p, q)$-Higgs Bundles}

Thesis submitted in partial fulfillment of the requirements for the degree of European Doctor in Mathematics, supervised by Dr. Oscar García Prada 

A mi familia y a mi abogado 



\section{Acknowledgements}

First of all, my gratitude goes to my advisor Oscar García Prada for giving me the opportunity of doing this thesis and for his help during all these years.

I want to thank Peter Gothen for his generous help, for encourage me and for being so patient with my mathematics and my English. Many thanks to Nigel Hitchin for showing me how complex things can be made simple and for many relevant discussions and e-mails. Thanks also to Ignasi Mundet i Riera for many discussions and for helping me with the stability.

I am very grateful to Luis Álvarez Cónsul for our discussions about mathematics and about the pros and cons of the researcher life. Many thanks to Marina Logares and André Oliveira for being my mathematical elder sister and brother, and to my workmates, Roberto Rubio, Mario García, Emilio Franco, and specially to Álvaro Antón, for their help and encouragement.

I thank to Instituto de Matemáticas y Física Fundamental and Instituto de Ciencias Matemticas (CSIC) for supporting this research and to Universidad de Barcelona, Universidade do Porto and Oxford University for the hospitality during my visits.

Finally, I want to thank my family and all my friends, some of them already mentioned, without their help I would not be able to finish this thesis. 



\section{Contents}

$\begin{array}{ll}\text { Introduction } & 1\end{array}$

$\begin{array}{lll}1 & \text { G-Higgs bundles } & 11\end{array}$

1.1 Parabolic subgroups $\ldots \ldots \ldots \ldots \ldots \ldots$

$1.2 \quad$ Stability $\ldots \ldots \ldots \ldots \ldots \ldots$

1.3 Stability in terms of filtrations . . . . . . . . . . . . . . . . . . . 14

$\begin{array}{lll}2 & \mathrm{SO}(n, \mathbb{C}) \text {-Higgs bundles } & 17\end{array}$

$2.1 \quad$ Parabolic subgroups of $\mathrm{SO}(n, \mathbb{C})$ and filtrations $\ldots \ldots \ldots . . \ldots 17$

2.2 Stability of $\mathrm{SO}(n, \mathbb{C})$-Higgs bundles $\ldots \ldots \ldots \ldots \ldots$. . . . . . . . 21

\begin{tabular}{lll}
\hline 3 & $\mathrm{SO}_{0}(p, q)$-Higgs bundles & 29
\end{tabular}

$3.1 \quad$ Stability of $\mathrm{SO}_{0}(p, q)$-Higgs bundles . . . . . . . . . . . . . . . 31

$3.2 \quad$ Polystable $\mathrm{SO}_{0}(p, q)$-Higgs bundles $\ldots \ldots \ldots \ldots$

3.3 Moduli space of $\mathrm{SO}_{0}(p, q)$-Higgs bundles. . . . . . . . . . . . . . . . . 42

$3.4 \quad \mathrm{SO}_{0}(p, q)$ - and $\mathrm{SO}(p+q, \mathbb{C})$-Higgs bundles stability $\ldots \ldots \ldots$. . . . 43

3.5 Smoothness and deformation theory . . . . . . . . . . . . . . . . . . . . 44

$3.6 \quad$ Stable but non-simple $\mathrm{SO}(n)$ and $\mathrm{SO}_{0}(p, q)$-Higgs bundles . . . . . . . . . . 47

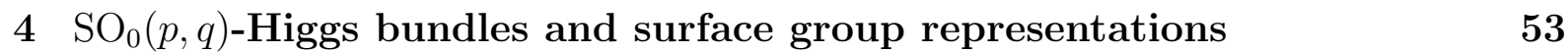

4.1 Representations and flat connections . . . . . . . . . . . . . . . . . 54

4.2 Harmonic reductions and harmonic equations . . . . . . . . . . . . . 56

4.3 Hitchin's equations and Higgs bundles . . . . . . . . . . . . . . . . . . . 62

4.4 The correspondence . . . . . . . . . . . . . . . . . . . . . . 66

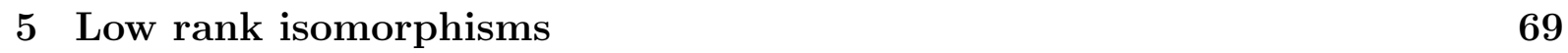

$5.1 \quad$ Isomorphisms of $\mathrm{SO}(3, \mathbb{C})$ and its real forms $\ldots \ldots \ldots . . \ldots 70$

5.2 Isomorphisms of $\mathrm{SO}(4, \mathbb{C})$ and its real forms $\ldots \ldots \ldots$. . . . . . . 72

5.3 Isomorphisms of $\mathrm{SO}(5, \mathbb{C})$ and its real forms $\ldots \ldots \ldots . . \ldots 76$

5.4 Isomorphisms of $\mathrm{SO}(6, \mathbb{C})$ and its real forms $\ldots \ldots \ldots$. . . . . . 81 
$5.5 \quad \mathrm{SO}_{0}(2,4)$-Higgs bundles and $\mathrm{SO}^{*}(8)$-Higgs bundles . . . . . . . . . . . 86

\begin{tabular}{lll}
\hline 6 & Smooth minima & 87
\end{tabular}

6.1 The Hitchin function $\ldots \ldots \ldots$. . . . . . . . . . . . . . . . . . . . 87

6.2 Morse Theory . . . . . . . . . . . . . . . . . . . . . . . . . . . 91

6.3 Critical points of the Hitchin function $\ldots \ldots \ldots$. . . . . . . . . . . . 92

6.4 Criterion for minima $\ldots \ldots \ldots \ldots \ldots$

6.5 Smooth minima . . . . . . . . . . . . . . . . . . . . . . . 101

\begin{tabular}{lll}
\hline 7 & Non-smooth minima & 117
\end{tabular}

$7.1 \quad$ Minima in $\mathcal{M}\left(\mathrm{SO}_{0}(3,3)\right) \ldots \ldots \ldots \ldots \ldots$

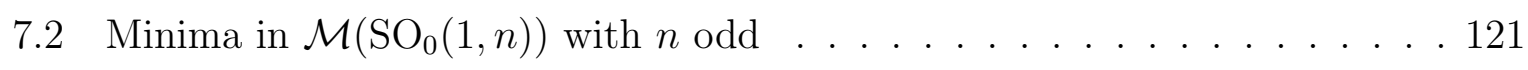

$\begin{array}{lll}8 & \text { The Hitchin component } & 123\end{array}$

8.1 Hitchin component for $\mathrm{SL}(2, \mathbb{R})$. . . . . . . . . . . . . . . 123

8.2 The general construction . . . . . . . . . . . . . . . . . . . . . 124

$8.3 \quad$ Hitchin component for $\mathrm{SL}(n, \mathbb{R}) \ldots \ldots \ldots$. . . . . . . . . 126

8.4 Hitchin component for $\mathrm{SO}_{0}(n, n)$. . . . . . . . . . . . 130

8.5 Hitchin component for $\mathrm{SO}_{0}(n, n+1) \ldots \ldots \ldots$. . . . . . . . 140

\begin{tabular}{ll}
\hline References & 145
\end{tabular} 


\section{Introduction}

Higgs bundles were introduced by Hitchin [31, 32] and are of interest in many areas of differential and algebraic geometry, topology and mathematical physics, such as the study of surface group representations, gauge theory, Kähler and hyperkähler geometry and integrable systems.

A Higgs bundle over a compact Riemann surface $X$ is a pair $(V, \phi)$ consisting of a holomorphic vector bundle $V$ over $X$ and a holomorphic section $\phi$ of $\operatorname{End}(V) \otimes K$, where $K$ is the canonical line bundle over $X$. The section $\phi$ is called the Higgs field. A Higgs bundle is said to be stable if for each subbundle $U \subset V$ for which $\phi(U) \subseteq U \otimes K$, one has

$$
\frac{\operatorname{deg}(U)}{\operatorname{rk}(U)}<\frac{\operatorname{deg}(V)}{\operatorname{rk}(V)} .
$$

Semistability is defined replacing the strict inequality with a weak inequality.

The notion of Higgs bundle can be extended to the notion of $G$-Higgs bundle, where $G$ is a complex reductive Lie group. A $G$-Higgs bundle is then a pair $(E, \varphi)$ where $E$ is a principal $G$-bundle over $X$ and $\varphi$ is a holomorphic section of the vector bundle $E(\mathfrak{g})=E \times{ }_{\text {Ad }} \mathfrak{g}$ twisted by $K$. When $G=\operatorname{GL}(n, \mathbb{C})$, the standard representation of $\operatorname{GL}(n, \mathbb{C})$ in $\mathbb{C}^{n}$ gives a one-to-one correspondence between principal $\operatorname{GL}(n, \mathbb{C})$-bundles and vector bundles of rank $n$. Using this correspondence we have that $E(\mathfrak{g l}(n, \mathbb{C}))=\operatorname{End}(V)$, where $V$ is the vector bundle associated to the principal $\operatorname{GL}(n, \mathbb{C})$-bundle $E$. Hence, this notion is equivalent to the notion of Higgs bundle described above. Simpson [51, 52] studied Higgs bundles in higher dimension and considered $G$-Higgs bundles for arbitrary complex reductive Lie groups $G$, which were also studied by Hitchin [30, 32] on Riemann surfaces.

The extension of the theory of $G$-Higgs bundles to real reductive Lie groups has been systematically studied by Bradlow, García-Prada, Gothen and Mundet i Riera (see for example [7, 19]). Let $G$ be a real reductive Lie group, $H \subseteq G$ be a maximal compact subgroup and $H^{\mathbb{C}}$ be its complexification. Let $\iota: H^{\mathbb{C}} \rightarrow \mathrm{GL}\left(\mathfrak{m}^{\mathbb{C}}\right)$ be the complexified isotropy representation defined in terms of the Cartan decomposition, $\mathfrak{g}=\mathfrak{h}+\mathfrak{m}$, of the Lie algebra of $G$ and using the fact that $[\mathfrak{h}, \mathfrak{m}] \subseteq \mathfrak{m}$. A $G$-Higgs bundle is a pair $(E, \varphi)$ where $E$ is a principal $H^{\mathbb{C}}$-bundle over $X$ and $\varphi$ is a holomorphic section of the vector bundle $E\left(\mathfrak{m}^{\mathbb{C}}\right) \otimes K=\left(E \times \mathfrak{m}^{\mathbb{C}}\right) \otimes K$. If $G$ is a complex reductive Lie group then, applying this definition to the underlying real Lie group, we recover the notion of $G$-Higgs bundle for a complex Lie group $G$.

The moduli space of Higgs bundles were constructed by Nitsure [42] using Geome- 
tric Invariant Theory. The moduli space $\mathcal{M}(G)$ of $G$-Higgs bundles was constructed by Simpson [53, 54] for a complex reductive Lie group $G$ using algebraic methods and in general (including real reductive Lie groups) by Schmitt [49, 50] using Geometric Invariant Theory.

$G$-Higgs bundles are important in relation to the representations of the fundamental group of the surface $X$. Let $G$ be a reductive Lie group, the moduli space of representations of $\pi_{1}(X)$ in $G$ is defined as the orbit space

$$
\mathcal{R}(G)=\operatorname{Hom}^{+}\left(\pi_{1}(X), G\right) / G
$$

where $\operatorname{Hom}^{+}\left(\pi_{1}(X), G\right)$ denotes the subspace of all reductive representations of $\pi_{1}(X)$ on $G$, that is, those that composed with the adjoint representation in the Lie algebra of $G$ decompose as a sum of irreducible representations, and $G$ acts on $\operatorname{Hom}^{+}\left(\pi_{1}(X), G\right)$ by conjugation. The space $\mathcal{R}(G)$ is Hausdorff with the quotient topology and it is a real analytic variety (see Goldman [22]).

Moduli spaces of representations have a very rich topology and geometry, reflecting both properties of $X$ and $G$. They have been studied from many points of view. When $G$ is compact they appear, for example, in quantum field theories [3]. If $G=\mathrm{U}(n)$, then the theorem of Narasimhan and Seshadri [41] gives an homeomorphism between $\mathcal{R}(\mathrm{U}(n))$ and the moduli space of semistable holomorphic vector bundles of rank $n$ and degree 0 . This result is generalized by Ramanathan [47] for any compact Lie group $G$. He proved that, when $G$ is semisimple, the moduli space of representations $\mathcal{R}(G)$, when $G$ is semisimple, is homeomorphic to the moduli space of semistable principal $G^{\mathbb{C}}$-bundles on $X$, where $G^{\mathbb{C}}$ is the complexification of $G$. If $G$ is reductive, there is an analogous result that involves the representations of the universal central extension of the fundamental group of $X$ on $G$.

There is a homeomorphism between $\mathcal{R}(G)$ and $\mathcal{M}(G)$ for any reductive real Lie group $G$ that provides a generalization for non-compact groups of the theorem of Narasimhan and Seshadri and the results of Ramanathan mentioned above. The proof of this result involves the moduli space of solutions to the Hitchin's equations. It was proved by Hitchin [31] and by Simpson [52, 53, 54] for a complex Lie group and by Bradlow, García-Prada, Gothen and Mundet i Riera [10, 19] in the real case, that $\mathcal{M}(G)$ is homeomorphic to the moduli space of solutions to the Hitchin's equations, $\mathcal{M}^{\text {Hit }}(G)$, which is defined as the space of pairs $(A, \varphi)$, where $A$ is a connection on a smooth principal $H$-bundle $E_{H}$ and 
$\varphi \in \Omega^{1,0}\left(E_{H}\left(\mathfrak{m}^{\mathbb{C}}\right)\right)$, satisfying

$$
\begin{aligned}
F_{A}-[\varphi, \tau(\varphi)] & =0 \\
\bar{\partial}_{A}(\varphi) & =0
\end{aligned}
$$

modulo gauge equivalence. The other important ingredient involved in the proof of the homeomorphism $\mathcal{R}(G) \cong \mathcal{M}(G)$ is a theorem of Corlette [13], and Donaldson [15] for $G=\mathrm{SL}(2, \mathbb{C})$, that establishes a homeomorphism between $\mathcal{R}(G)$ and the moduli space $\mathcal{M}^{\text {Har }}(G)$ of solutions of certain harmonic equations, defined as the space of pairs $(A, \psi)$, where $A$ is a connection on a smooth principal $H$-bundle $E_{H}$ and $\psi \in \Omega^{1}\left(E_{H}(\mathfrak{m})\right)$, satisfying

$$
\begin{aligned}
F_{A}+\frac{1}{2}[\psi, \psi] & =0 \\
d_{A}(\psi) & =0 \\
d_{A}^{*}(\psi) & =0
\end{aligned}
$$

modulo gauge equivalence. The map $(A, \varphi) \mapsto(A, \psi=\varphi-\tau(\varphi))$ defines a homeomorphism between $\mathcal{M}^{\text {Hit }}(G)$ and $\mathcal{M}^{\text {Har }}(G)$ and gives $\mathcal{R}(G) \cong \mathcal{M}(G)$. Using these homeomorphisms one can obtain information about the moduli space of representations working with $\mathcal{M}(G)$ or with the moduli space of solutions of Hitchin's equations.

The geometry and topology of the moduli space of $G$-Higgs bundles have been studied for several complex reductive Lie groups like $\operatorname{GL}(n, \mathbb{C}), \operatorname{SL}(n, \mathbb{C}), \operatorname{SO}(n, \mathbb{C})$ and $\operatorname{Sp}(2 n, \mathbb{C})$, and for some of their real forms, like $\mathrm{U}(p, q), \operatorname{Sp}(2 n, \mathbb{R})$, among others. This thesis is devoted to the study of the moduli space of $\mathrm{SO}_{0}(p, q)$-Higgs bundles, where $\mathrm{SO}_{0}(p, q)$ is the connected component of the identity of the Lie group $\mathrm{SO}(p, q)$. The special orthogonal group with signature $(p, q), \mathrm{SO}(p, q)$, is the subgroup of $\mathrm{SL}(n=p+q, \mathbb{R})$ consisting of all linear transformations of a $n=p+q$ dimensional real vector space which leave invariant a non-degenerate symmetric bilinear form of signature $(p, q)$ and it is a real form of the reductive group $\mathrm{SO}(p+q, \mathbb{C})$. It coincides with the compact real form when $p$ or $q$ are equal to zero and with the split form when $p=q$, if $p+q$ is even, or when $q=p+1$, if $p+q$ is odd. The Cartan decomposition of the complex Lie algebra $\mathfrak{s o}(p+q, \mathbb{C})$ is

$$
\mathfrak{s o}(p+q, \mathbb{C})=(\mathfrak{s o}(p, \mathbb{C}) \times \mathfrak{s o}(q, \mathbb{C})) \oplus \mathfrak{m}^{\mathbb{C}}
$$

where

$$
\mathfrak{m}^{\mathbb{C}}=\left\{\left(\begin{array}{cc}
X_{2} \\
-X_{2}^{t}
\end{array}\right) \mid X_{2} \text { complex }(p \times q) \text {-matrix }\right\} .
$$

Following the definition given above, an $\mathrm{SO}_{0}(p, q)$-Higgs bundle is a pair $(E, \varphi)$ consisting of a holomorphic principal $\mathrm{SO}(p, \mathbb{C}) \times \mathrm{SO}(q, \mathbb{C})$-bundle $E$ and a section $\varphi \in$ $H^{0}\left(E\left(\mathfrak{m}^{\mathbb{C}}\right) \otimes K\right)$. Using the standard representations of $\operatorname{SO}(p, \mathbb{C})$ and $\operatorname{SO}(q, \mathbb{C})$ in $\mathbb{C}^{p}$ 
and $\mathbb{C}^{q}$ respectively, there is a one-to-one correspondence between $\mathrm{SO}_{0}(p, q)$-Higgs bundles and tuples $\left(V, Q_{V}, W, Q_{W}, \eta\right)$, where $V$ and $W$ are two holomorphic vector bundles of rank $p$ and $q$ respectively and trivial determinant, $Q_{V}$ and $Q_{W}$ are non-degenerate symmetric quadratic forms in $V$ and $W$ respectively and $\eta \in H^{0}(\operatorname{Hom}(W, V) \otimes K)$. To an $\mathrm{SO}_{0}(p, q)$-Higgs bundle $\left(V, Q_{V}, W, Q_{W}, \eta\right)$ we can associate an $\mathrm{SO}(p+q, \mathbb{C})$-Higgs bundle defined by the triple

$$
\left(\mathbb{E}=V \oplus W, Q=\left(\begin{array}{ll}
Q_{V} & \\
& Q_{W}
\end{array}\right), \phi=\left(\begin{array}{cc} 
& \eta \\
& -\eta^{\top}
\end{array}\right)\right) .
$$

In this thesis we study the notions of semistability, stability and polystability for $\mathrm{SO}(n, \mathbb{C})$ and $\mathrm{SO}_{0}(p, q)$-Higgs bundles applying the general notions given by García-Prada, Gothen and Mundet i Riera [19], that generalize the results given by Ramanathan [47] for principal bundles. We show that the notions of stability and semistability for $\operatorname{SO}(n, \mathbb{C})$ and $\mathrm{SO}_{0}(p, q)$-Higgs bundles can be simplified to the following.

Proposition (see Proposition 2.4). An $\mathrm{SO}(n, \mathbb{C})$-Higgs bundle $(\mathbb{E}, Q, \phi)$ with $n \neq 2$ is semistable if and only if for any isotropic subbundle $E^{\prime} \subset \mathbb{E}$ such that $\phi\left(E^{\prime}\right) \subseteq E^{\prime} \otimes K$ the inequality $\operatorname{deg} E^{\prime} \leq 0$ holds, and it is stable if it is semistable and for any non-zero isotropic subbundle $E^{\prime} \subset \mathbb{E}$ such that $\phi\left(E^{\prime}\right) \subseteq E^{\prime} \otimes K$ we have $\operatorname{deg} E^{\prime}<0$.

Proposition (see Proposition 3.3). An $\mathrm{SO}_{0}(p, q)$-Higgs bundle $\left(V, Q_{V}, W, Q_{W}, \eta\right)$ with $p, q \neq 2$ is semistable if and only if for any pair of isotropic subbundles $V^{\prime} \subset V, W^{\prime} \subset W$ such that $\eta\left(W^{\prime}\right) \subseteq V^{\prime} \otimes K$, the inequality $\operatorname{deg} V^{\prime}+\operatorname{deg} W^{\prime} \leq 0$ holds. It is stable if and only if it is semistable and for any pair of isotropic subbundles $V^{\prime} \subset V, W^{\prime} \subset W$, at least one of them non-zero, such that $\eta\left(W^{\prime}\right) \subseteq V^{\prime} \otimes K$, we have $\operatorname{deg} V^{\prime}+\operatorname{deg} W^{\prime}<0$.

The cases $n=2$ and $p=2$ or $q=2$ require an special treatment. Every $\mathrm{SO}(2, \mathbb{C})$-Higgs bundle is semistable and stable. The simplified notions of semistability and stability for $\mathrm{SO}_{0}(p, q)$-Higgs bundles with $p=2$ (resp. $q=2$ ) involves only the isotropic subbundles of $W($ resp. $V)$.

Morse-theoretic techniques for studying the topology of moduli spaces of Higgs bundles were introduced by Hitchin [31, 32]. The problem of counting the connected components of $\mathcal{M}(G)$ using these methods has been carried out for several reductive real Lie groups. Hitchin solved the problem for the groups $\operatorname{SL}(n, \mathbb{R})$ and $\operatorname{PSL}(n, \mathbb{R})$ in 32 . His methods were extended to $\mathrm{U}(p, q)$ and $\mathrm{GL}(n, \mathbb{R})$ with $n \geq 3$ by Bradlow, García-Prada and Gothen in [7, 8]. The problem for the symplectic group $\operatorname{Sp}(4, \mathbb{R})$ was studied by Gothen in [25] and by García-Prada and Mundet i Riera in [21], whereas the general case $\operatorname{Sp}(2 n, \mathbb{R})$ was 
studied by García-Prada, Gothen and Mundet i Riera in [19]. The case of $\operatorname{PGL}(n, \mathbb{R})$ has been studied recently by Oliveira in [43].

In this thesis we give important steps in the program of counting the connected components of the moduli space $\mathcal{M}(G)$ for $G=\mathrm{SO}_{0}(p, q)$. The classification of principal $\mathrm{SO}(p, \mathbb{C})$ and $\mathrm{SO}(q, \mathbb{C})$-bundles defines a topological invariant $c=\left(c_{1}, c_{2}\right) \in \pi_{1}(\mathrm{SO}(p, \mathbb{C})) \times$ $\pi_{1}(\mathrm{SO}(q, \mathbb{C}))$ in the moduli space of polystable $\mathrm{SO}_{0}(p, q)$-Higgs bundles. When $p, q \geq 3$, the invariant $\left(c_{1}, c_{2}\right) \in \mathbb{Z}_{2} \times \mathbb{Z}_{2}$ corresponds to the second Stiefel-Whitney classes of the two orthogonal bundles that we obtain from the reduction of the structure groups of $\left(V, Q_{V}\right)$ and $\left(W, Q_{W}\right)$ from $\mathrm{SO}(p, \mathbb{C})$ and $\mathrm{SO}(q, \mathbb{C})$ to the real groups $\mathrm{SO}(p)$ and $\mathrm{SO}(q)$. This gives a first decomposition of the moduli space

$$
\mathcal{M}(G)=\coprod_{c} \mathcal{M}_{c}(G)
$$

To obtain the number of connected components it is necessary to distinguish which of these components $\mathcal{M}_{c}(G)$ are connected and which decompose as a union of connected components. The strategy of Hitchin for finding the connected components in $\mathcal{M}_{c}(G)$ is to consider the Hitchin function

$$
\begin{aligned}
f: \mathcal{M}_{c}(G) & \rightarrow \mathbb{R} \\
{[A, \varphi] } & \mapsto\|\varphi\|^{2},
\end{aligned}
$$

where one identifies the moduli space of $G$-Higgs bundles with the space of solutions to Hitchin's equations and we use the $L^{2}$-norm $\|\cdot\|$ defined by the metric that solves the Hitchin's equations. It is a consequence of the Uhlenbeck's weak compactness theorem that this function is proper [31], and so it must have a minimum on each connected component of $\mathcal{M}_{c}(G)$.

To apply these techniques, the first step is to compute the critical points of $f$. The Hitchin function $f$ is the moment map of the circle action $[A, \varphi] \mapsto\left[A, e^{i \theta} \varphi\right]$, when we restrict to the smooth locus, $\mathcal{M}_{c}^{s}(G)$. This implies that a smooth point of the moduli space is a critical point if and only if it is a fixed point of the circle action. If $[A, \varphi] \in \mathcal{M}_{c}(G)$, it is a fixed point of the circle action if and only if there exists a 1-parameter family of gauge transformations $\{g(\theta)\}$, generated by an infinitesimal gauge transformation $\psi$, such that $d_{A}(\psi)=0$ and $[\psi, \varphi]=i \varphi$.

A $G$-Higgs bundle $(E, \varphi)$ associated to the solution $(A, \varphi)$ corresponds to a fixed point of the circle action if and only if it is a Hodge bundle (also called a complex variation of Hodge structure, cf. [51]). This means that the vector bundles $E\left(\mathfrak{h}^{\mathbb{C}}\right)$ and $E\left(\mathfrak{m}^{\mathbb{C}}\right)$ 
decompose in sums of eigenbundles for the action of the infinitesimal transformation $\psi$ and that $[\psi, \varphi]=i \varphi$.

Once we have characterized the critical points of $f$, the second step is to identify which are the minima, that is, to find the smooth Hodge bundles for which the Hessian is non-negative definite. This can be done using the method given by Hitchin in [32, §8]. Let

$$
C^{\bullet}(E, \varphi): E\left(\mathfrak{h}^{\mathbb{C}}\right) \stackrel{\operatorname{ad}(\varphi)}{\longrightarrow} E\left(\mathfrak{m}^{\mathbb{C}}\right) \otimes K
$$

be the deformation complex (see [19]), whose first hypercohomology group $\mathbb{H}^{1}\left(C^{\bullet}(E, \varphi)\right.$ ) is the space of infinitesimal deformations of $(E, \varphi)$. When $(E, \varphi)$ represents a smooth point, $\mathbb{H}^{1}\left(C^{\bullet}(E, \varphi)\right)$ is canonically isomorphic to the tangent space at this point and, for all eigenvalues $k$ of $\psi$, the hypercohomology group $\mathbb{H}^{1}\left(C_{k}^{\bullet}(E, \varphi)\right)$, where

$$
C_{k}^{\bullet}(E, \varphi): E\left(\mathfrak{h}^{\mathbb{C}}\right)_{k} \stackrel{\operatorname{ad}(\varphi)}{\longrightarrow} E\left(\mathfrak{m}^{\mathbb{C}}\right)_{k+1} \otimes K
$$

is isomorphic to the eigenspace of the Hessian of $f$ with eigenvalue $-k$. Then, a smooth $G$-Higgs bundle $(E, \varphi)$ is a minimum if and only if $\mathbb{H}^{1}\left(C_{k}^{\bullet}(E, \varphi)\right)=0$ for $k>0$.

If this condition holds, even when the $G$-Higgs bundle $(E, \varphi)$ is not smooth, we continue to have a local minimum. Following Hitchin's method, to rule out non-smooth $G$-Higgs bundles as minima it is necessary to find a smooth family of deformations of $(E, \varphi)$ such that the corresponding infinitesimal deformation is a non-zero element of $\mathbb{H}^{1}\left(C_{k}^{\bullet}(E, \varphi)\right)$ for some $k>0$.

In this thesis we apply these methods to the moduli space of polystable $\mathrm{SO}_{0}(p, q)$ Higgs bundles. Our main result is Theorem 6.10 which gives a complete description of the smooth minima of the Hitchin function in the moduli space of $\mathrm{SO}_{0}(p, q)$-Higgs bundles. We also solve the problem of finding the number of connected components of the moduli space of polystable $\mathrm{SO}_{0}(1, n)$-Higgs bundles with $n$ odd.

Theorem (see Theorem 7.5). The moduli space of $\mathrm{SO}_{0}(1, n)$-Higgs bundles with $n$ odd has 2 connected components.

Among the real forms $\mathrm{SO}_{0}(p, q)$, the split real forms $\mathrm{SO}_{0}(n, n)$ and $\mathrm{SO}_{0}(n, n+1)$ play and important role. It was proved by Hitchin in [32] that, when $G$ is the split real form of some complex reductive Lie group, the moduli space $\mathcal{M}(G)$ has a connected component homeomorphic to a Euclidean space of dimension $(2 g-2) \operatorname{dim} G$. This component is called the Hitchin component. When $G=\operatorname{PSL}(2, \mathbb{R})$, this component can be identified with Teichmüller space. In [32, §5] he gives a general method to construct Hitchin component which is based on the definition of a section of the Hitchin map. We revisit the general 
method given by Hitchin in 32 to construct Hitchin component and specialize it to the orthogonal split real forms $\mathrm{SO}_{0}(n, n)$ and $\mathrm{SO}_{0}(n, n+1)$.

Using the characterizations of simple and smooth $G$-Higgs bundle given by GarcíaPrada, Gothen and Mundet i Riera in [19], and the relation between the stability of an $\mathrm{SO}_{0}(p, q)$-Higgs bundle and the stability of its associated $\mathrm{SO}(p+q, \mathbb{C})$-Higgs bundle, we prove that an $\mathrm{SO}_{0}(p, q)$-Higgs bundle is smooth if and only if it is stable and simple. Hence, stable does not imply simple in the moduli space of $\mathrm{SO}_{0}(p, q)$-Higgs bundles. This is an specific phenomenon of the complex Lie groups $\mathrm{SO}(n, \mathbb{C})$ and $\mathrm{Sp}(2 n, \mathbb{C})$, and their real forms, that appears already for principal bundles (see [46, 47]). This means that a polystable $\mathrm{SO}_{0}(p, q)$-Higgs bundle fails to be smooth if it is stable but non-simple, or if it is strictly polystable.

Even when the $\mathrm{SO}_{0}(p, q)$-Higgs bundle is not smooth, if $\mathbb{H}^{1}\left(C_{k}^{\bullet}\right)=0$ for all $k>0$, it is a local minimum. Then, after giving a description of the stable but non-simple $\mathrm{SO}_{0}(p, q)$ Higgs bundles, we see that we can include in a more general theorem about minima the stable but non-simple elements that satisfy this condition. We conjecture that these are all the possible stable minima in $\mathcal{M}\left(\mathrm{SO}_{0}(p, q)\right)$. To prove this, we should rule out some non-smooth $\mathrm{SO}_{0}(p, q)$-Higgs bundles as minima. This turns out to be technically much more difficult than the previous studied cases and this is calling for new methods to be developed.

Another topic treated in this thesis is the isomorphisms for special orthogonal groups of low rank. Cartan proved in [12] the existence of a list of isomorphisms between some classical semisimple Lie algebras of low rank (see [29, Ch. X, §6]):

$$
\begin{aligned}
\mathfrak{s o}(3, \mathbb{C}) & \cong \mathfrak{s l}(2, \mathbb{C}) \cong \mathfrak{s p}(2, \mathbb{C}), \\
\mathfrak{s o}(3) & \cong \mathfrak{s u}(2) \cong \mathfrak{s p}(2), \\
\mathfrak{s o}(2,1) & \cong \mathfrak{s l}(2, \mathbb{R}) \\
\mathfrak{s o}(4, \mathbb{C}) & \cong \mathfrak{s l}(2, \mathbb{C}) \times \mathfrak{s l}(2, \mathbb{C}), \\
\mathfrak{s o}(4) & \cong \mathfrak{s u}(2) \times \mathfrak{s u}(2), \\
\mathfrak{s o}(2,2) & \cong \mathfrak{s l}(2, \mathbb{R}) \times \mathfrak{s l}(2, \mathbb{R}), \\
\mathfrak{s o}(1,3) & \cong \mathfrak{s l}(2, \mathbb{C}), \\
\mathfrak{s o}(2,6) & \cong \mathfrak{s o}^{*}(8),
\end{aligned}
$$

$$
\begin{aligned}
\mathfrak{s o}(5, \mathbb{C}) & \cong \mathfrak{s p}(4, \mathbb{C}), \\
\mathfrak{s o}(5) & \cong \mathfrak{s p}(4), \\
\mathfrak{s o}(2,3) & \cong \mathfrak{s p}(4, \mathbb{R}), \\
\mathfrak{s o}(1,4) & \cong \mathfrak{s p}(2,2), \\
\mathfrak{s o}(6, \mathbb{C}) & \cong \mathfrak{s l}(4, \mathbb{C}), \\
\mathfrak{s o}(6) & \cong \mathfrak{s u}(4), \\
\mathfrak{s o}(3,3) & \cong \mathfrak{s l}(4, \mathbb{R}), \\
\mathfrak{s o}(2,4) & \cong \mathfrak{s u}(2,2), \\
\mathfrak{s o}(1,5) & \cong \mathfrak{s u}(4),
\end{aligned}
$$

Using these isomorphisms and the list of isomorphisms between Spin Lie groups and other classical semisimple Lie groups described in [28, Theorem 14.1], we study the relation 
between the moduli space of polystable $\mathrm{SO}(n, \mathbb{C})$-Higgs bundles with $n=3,4,5$ and 6 and the moduli space of polystable $G$-Higgs bundles, where $G=\operatorname{SL}(2, \mathbb{C}), \operatorname{SL}(2, \mathbb{C}) \times \operatorname{SL}(2, \mathbb{C})$, $\operatorname{Sp}(4, \mathbb{C})$ and $\mathrm{SL}(4, \mathbb{C})$ respectively. We also described the relations between the real forms $\mathrm{SO}_{0}(p, q)$ with $p+q=n$, for $n=3,4,5,6$, and the corresponding real forms of the Lie group $G$, describing explicitly the morphisms between the moduli spaces of Higgs bundles.

Now we give a description of the different chapters of this thesis.

In Chapter 1 we review the definition of $G$-Higgs bundle, where $G$ is a real Lie group. This chapter also includes the definition of the stability condition for a $G$-Higgs bundle introduced in [10, 19], and sketches the basic ingredients that it involves, such as parabolic subgroups, Levi subgroups, antidominant characters, reduction of structure group and filtrations.

In Chapter 2 we apply the general notions to the special orthogonal complex Lie group $\mathrm{SO}(n, \mathbb{C})$. In order to make easier the understanding of the notions of semistability, stability and polystability, we develop the case $n=6$, which is a simple but rich enough example to illustrate this theory.

We introduce the definition of $\mathrm{SO}_{0}(p, q)$-Higgs bundle in Chapter 3. As in the case of $\mathrm{SO}(n, \mathbb{C})$, we apply the general notions to obtain the notions of semistability, stability and polystability for $\mathrm{SO}_{0}(p, q)$-Higgs bundles and we give a simplified notion of stability in Proposition 3.3. We illustrate the theory with an example that explains in detail the stability for $\mathrm{SO}_{0}(3,3)$-Higgs bundles. In Section 3.5 we prove that an $\mathrm{SO}_{0}(p, q)$-Higgs bundle is smooth if and only if it is stable and simple. The chapter concludes with a full description of the two types of non-smooth objects, stable but non-simple and strictly polystable $\mathrm{SO}_{0}(p, q)$-Higgs bundles, that will be useful in the study of the non-smooth minima carried out in Chapter 8.

In Chapter 4, we describe the principal objects and results involved in the homeomorphism $\mathcal{R}(G) \cong \mathcal{M}(G)$, particularizing to the case $G=\mathrm{SO}_{0}(p, q)$.

In Chapter 5, we describe the maps that occur between several moduli spaces of Higgs bundles induced by the isomorphisms between low rank orthogonal Lie algebras and other reductive Lie algebras given by Cartan in [12].

The main result of this thesis is proved in Chapter 6. We give an explicit description of the smooth minima of the Hitchin function in $\mathcal{M}\left(\mathrm{SO}_{0}(p, q)\right)$ (Theorem 6.10). We also study in detail the general techniques used to prove the theorem. We define the Hitchin function and explain the role that it plays in the study of the topology of $\mathcal{M}\left(\mathrm{SO}_{0}(p, q)\right)$. We also describe the critical points and explain the criterion of minima applied in the 
smooth case.

In Chapter 7 we explain the general methods given by Hitchin for the study of the non-smooth minima of the Hitchin function, we conjecture what happens with the nonsmooth minima in $\mathcal{M}\left(\mathrm{SO}_{0}(p, q)\right)$ and we explain the technical problems that appear in this case. We finally compute the connected components of the moduli space of polystable $\mathrm{SO}_{0}(1, n)$-Higgs bundles with $n$ odd.

Chapter 8 is devoted to the construction of the Hitchin components of the moduli spaces $\mathcal{M}\left(\mathrm{SO}_{0}(n, n)\right)$ and $\mathcal{M}\left(\mathrm{SO}_{0}(n, n+1)\right)$. We revisit the general method given by Hitchin in [32] and then specialize it to the special orthogonal split real forms. 



\section{$1 \quad G$-Higgs bundles}

Let $X$ be a compact Riemann surface. Let $G$ be a real reductive Lie group, $H$ be a maximal compact subgroup of $G$ and $H^{\mathbb{C}}$ be its complexification. Let

$$
\iota: H^{\mathbb{C}} \rightarrow \mathrm{GL}\left(\mathfrak{m}^{\mathbb{C}}\right),
$$

be the complexified isotropy representation, defined in terms of the Cartan decomposition $\mathfrak{g}=\mathfrak{h}+\mathfrak{m}$ of the Lie algebra of $G$ and using the fact that $[\mathfrak{h}, \mathfrak{m}] \subseteq \mathfrak{m}$.

Definition 1.1. A G-Higgs bundle is a pair $(E, \varphi)$ where $E$ is a principal $H^{\mathbb{C}}$-bundle over $X$ and $\varphi$ is a holomorphic section of the vector bundle $E\left(\mathfrak{m}^{\mathbb{C}}\right) \otimes K=\left(E \times{ }_{\iota} \mathfrak{m}^{\mathbb{C}}\right) \otimes K$, where $K$ is the canonical line bundle over $X$. The section $\varphi$ is called the Higgs field.

Two G-Higgs bundles $(E, \varphi)$ and $\left(E^{\prime}, \varphi^{\prime}\right)$ are isomorphic if there is an isomorphism $E \cong E^{\prime}$ which takes $\varphi$ to $\varphi^{\prime}$ under the induced isomorphism $E\left(\mathfrak{m}^{\mathbb{C}}\right) \cong E^{\prime}\left(\mathfrak{m}^{\mathbb{C}}\right)$.

When $G$ is a real compact reductive Lie group, the Cartan decomposition of the Lie algebra is $\mathfrak{g}=\mathfrak{h}$ and then the Higgs field is equal to zero. Hence, a $G$-Higgs bundle is in fact a principal $G^{\mathbb{C}}$-bundle.

Let $G$ be a complex reductive Lie group and consider the underlying real Lie group $G^{\mathbb{R}}$. In this case, the complexification $H^{\mathbb{C}}$ of a maximal compact subgroup is again the Lie group $G$ and since

$$
\mathfrak{g}^{\mathbb{R}}=\mathfrak{h}+i \mathfrak{h},
$$

the isotropy representation coincides with the adjoint representation of $G$ on its Lie algebra. Applying Definition 1.1 to $G^{\mathbb{R}}$ we recover the notion of $G$-Higgs bundle for a complex reductive Lie group $G$. When $G=\operatorname{GL}(n, \mathbb{C})$, the standard representation of $\operatorname{GL}(n, \mathbb{C})$ in $\mathbb{C}^{n}$ gives a one-to-one correspondence between principal $\operatorname{GL}(n, \mathbb{C})$-bundles and vector bundles of rank $n$. Using this correspondence we have $E(\mathfrak{g l}(n, \mathbb{C}))=\operatorname{End}(V)$, where $V$ is the vector bundle associated to the principal $\mathrm{GL}(n, \mathbb{C})$-bundle $E$. Hence, this notion recovers the notion of Higgs bundle.

A good reference for the material explained in the rest of the chapter is [19].

\subsection{Parabolic subgroups}

A subgroup $P \subset G$ is said to be parabolic if $G / P$ is a complete variety, that is, $G / P$ is an algebraic variety such that for any variety $Y$ the projection morphism $G / P \times Y \rightarrow Y$ is a closed map. The most common example of complete variety is a projective variety. 
Let $G$ be a complex semisimple Lie group and consider a Cartan subalgebra $\mathfrak{c}$ of the Lie algebra $\mathfrak{g}$. Let $\Delta$ be the set of roots of $\mathfrak{g}$ with respect to this Cartan subalgebra. We have a decomposition

$$
\mathfrak{g}=\mathfrak{c} \oplus \bigoplus_{\alpha \in \Delta} \mathfrak{g}_{\alpha}
$$

where $\mathfrak{g}_{\alpha}=\{H \in \mathfrak{g} \mid(\operatorname{ad} C) H=\alpha(C) H$, for all $C \in \mathfrak{c}\}$. Let $\Delta^{+}$be the set of positive roots and $\Pi=\left\{\alpha_{1}, \ldots, \alpha_{n}\right\}$ be the set of simple roots. For any subset $A \subset \Pi$ we define

$$
\Delta_{A}=\left\{\delta \in \Delta \mid \delta=\sum_{i=1}^{n} m_{i} \alpha_{i} \text { with } m_{i} \geq 0 \text { for all } \alpha_{i} \in A\right\},
$$

and let

$$
\mathfrak{p}_{A}=\mathfrak{c} \oplus \bigoplus_{\delta \in \Delta_{A}} \mathfrak{g}_{\delta}
$$

If $P_{A} \subset G$ is the connected subgroup with Lie algebra $\mathfrak{p}_{A}$, then $P_{A}$ is a parabolic subgroup of $G$. Similarly we can define

$$
\Delta_{A}^{0}=\left\{\delta \in \Delta \mid \delta=\sum_{i=1}^{n} m_{i} \alpha_{i} \text { with } m_{i}=0 \text { for all } \alpha_{i} \in A\right\},
$$

and let

$$
\mathfrak{l}_{A}=\mathfrak{c} \oplus \bigoplus_{\delta \in \Delta_{A}^{0}} \mathfrak{g}_{\delta}
$$

which is a subalgebra of $\mathfrak{p}_{A}$. If $L_{A}$ is the connected subgroup with Lie algebra $\mathfrak{l}_{A}$, then $L_{A}$ is a Levi subgroup of $P_{A}$.

A weight $\lambda \in \mathfrak{c}^{*}$ is said to be dominant if $\frac{2\left\langle\lambda, \alpha_{i}\right\rangle}{\left\langle\alpha_{i}, \alpha_{i}\right\rangle} \geq 0$ for all $\alpha_{i} \in \Pi$, where $\langle\cdot, \cdot\rangle$ denotes the Killing form of $\mathfrak{g}$, and it is said to be strictly dominant if $\frac{2\left\langle\lambda, \alpha_{i}\right\rangle}{\left\langle\alpha_{i}, \alpha_{i}\right\rangle}>0$ for all $\alpha_{i} \in \Pi$.

Every simple root $\alpha_{i} \in \Pi$, has an associated coroot defined by the formula

$$
\alpha_{i}^{\prime}=\frac{2}{\left\langle\alpha_{i}, \alpha_{i}\right\rangle} \alpha_{i}
$$

Let $\left\{\lambda_{1}, \ldots, \lambda_{n}\right\} \in \mathfrak{c}^{*}$ defined by the condition $\frac{2\left\langle\lambda_{i}, \alpha_{j}\right\rangle}{\left\langle\alpha_{j}, \alpha_{j}\right\rangle}=\delta_{i j}$. The $\lambda_{i}$ are dominant weights and we call them the fundamental dominant weights. Then, a weight $\lambda$ is dominant if $\lambda=\sum m_{i} \lambda_{i}$ with all $m_{i} \geq 0$, and strictly dominant if $\lambda=\sum m_{i} \lambda_{i}$ with all $m_{i}>0$.

An antidominant character for the parabolic subgroup $P_{A}$ is an element of the form

$$
\chi=\sum_{\alpha_{i} \in A} m_{i} \lambda_{i}
$$


with all $m_{i} \leq 0$. We say that $\chi$ is strictly antidominant if $m_{i}<0$ for all $\alpha_{i} \in A$.

If $G$ is a reductive group which is not semisimple, all these definitions can be generalized considering the semisimple part $\mathfrak{g}_{s}=[\mathfrak{g}, \mathfrak{g}]$ of $\mathfrak{g}$ and the decomposition

$$
\mathfrak{g}=\mathfrak{z} \oplus \mathfrak{g}_{s}
$$

where $\mathfrak{z}$ is the centre of $\mathfrak{g}$ (see [19]).

\section{$1.2 \quad$ Stability}

Let $(E, \varphi)$ be a $G$-Higgs bundle such that $H^{\mathbb{C}}$ is a semisimple complex Lie group, and consider a parabolic subgroup $P_{A} \subseteq H^{\mathbb{C}}$ and its Levi subgroup $L_{A} \subseteq P_{A}$.

To any antidominant character $\chi$ of $P_{A}$ we can associate an element $s_{\chi} \in i \mathfrak{h}$ taking the dual via the Killing form. Let

$$
\begin{aligned}
& P_{s_{\chi}}=\left\{g \in G \mid e^{t s_{\chi}} g e^{-t s_{\chi}} \text { is bounded as } t \rightarrow \infty\right\}, \\
& \mathfrak{p}_{s_{\chi}}=\left\{X \in \mathfrak{g} \mid \operatorname{Ad}\left(e^{t s_{\chi}}\right)(X) \text { is bounded as } t \rightarrow \infty\right\} .
\end{aligned}
$$

We have the inclusions $P_{s_{\chi}} \subseteq P_{A}$ and $\mathfrak{p}_{s_{\chi}} \subseteq \mathfrak{p}_{A}$ which are equalities when the antidominant character is strict. In fact, every parabolic subgroup of $H^{\mathbb{C}}$ is of the form $P_{s}$ with $s \in i \mathfrak{h}$.

A holomorphic section $\sigma$ of $E\left(H^{\mathbb{C}} / P_{A}\right)$ is equivalent to a reduction of the structure group of $E$ from $H^{\mathbb{C}}$ to $P_{A}$, that is, there exists a principal $P_{A}$-bundle $E_{\sigma}$ such that

$$
E \cong E_{\sigma} \times_{P_{A}} H^{\mathbb{C}}
$$

Analogously, if $\sigma_{L}$ is a holomorphic section of $E_{\sigma}\left(P_{A} / L_{A}\right)$, it gives a reduction of the structure group of $E_{\sigma}$ from $P_{A}$ to $L_{A}$,

$$
E_{\sigma} \cong E_{\sigma_{L}} \times_{L_{A}} P_{A}
$$

If $\chi$ is an antidominant character for $P_{A}$, let

$$
\begin{aligned}
& \left(\mathfrak{m}^{\mathbb{C}}\right)_{\chi}^{-}=\left\{v \in \mathfrak{m}^{\mathbb{C}} \mid \iota\left(e^{t s_{\chi}}\right) v \text { remains bounded as } t \rightarrow \infty\right\}, \\
& \left(\mathfrak{m}^{\mathbb{C}}\right)_{\chi}^{0}=\left\{v \in \mathfrak{m}^{\mathbb{C}} \mid \iota\left(e^{t s_{\chi}}\right) v=v \text { for any } t\right\} \subset\left(\mathfrak{m}^{\mathbb{C}}\right)_{\chi}^{-},
\end{aligned}
$$

which are subspaces of $\mathfrak{m}^{\mathbb{C}}$ invariant under the action of $P_{A}$ and $L_{A}$ respectively. We have that $E\left(\mathfrak{m}^{\mathbb{C}}\right) \cong E_{\sigma} \times_{P_{A}} \mathfrak{m}^{\mathbb{C}}$ and $E\left(\mathfrak{m}^{\mathbb{C}}\right) \cong E_{\sigma_{L}} \times_{L_{A}} \mathfrak{m}^{\mathbb{C}}$, and we can thus identify the vector bundles $E_{\sigma} \times_{P_{A}}\left(\mathfrak{m}^{\mathbb{C}}\right)_{\chi}^{-}$and $E_{\sigma_{L}} \times_{L_{A}}\left(\mathfrak{m}^{\mathbb{C}}\right)_{\chi}^{0}$ with two holomorphic subbundles

$$
E\left(\mathfrak{m}^{\mathbb{C}}\right)_{\chi}^{0} \subseteq E\left(\mathfrak{m}^{\mathbb{C}}\right)_{\chi}^{-} \subseteq E\left(\mathfrak{m}^{\mathbb{C}}\right)
$$


If $\chi=\sum_{\alpha_{i} \in A} m_{i} \lambda_{i}$, where $\left\{\lambda_{i}\right\} \in \mathfrak{c}^{*}$ is the set of fundamental weights associated to the simple roots $\Pi=\left\{\alpha_{i}\right\}$, there exists some positive integer $n$ such that for any $\alpha_{i} \in A$, the morphism of Lie algebras $n \lambda_{i}: \mathfrak{c} \rightarrow \mathbb{C}$ gives a morphism of Lie groups $\kappa_{n \alpha_{i}}: P_{A} \rightarrow \mathbb{C}^{*}$. (See [19], Lemma 2.3). We define the degree of the bundle $E$ with respect to a reduction $\sigma$ and to an antidominant character $\chi$ as the real number

$$
\operatorname{deg}(E)(\sigma, \chi)=\frac{1}{n} \sum_{\alpha_{i} \in A} \operatorname{deg}\left(E_{\sigma} \times_{\kappa_{n \alpha_{i}}} \mathbb{C}^{*}\right) .
$$

Definition 1.2. A G-Higgs bundle $(E, \varphi)$ is called semistable if for any parabolic subgroup $P \subset H^{\mathbb{C}}$, any antidominant character $\chi$ for $P$, and any holomorphic section $\sigma \in \Gamma\left(E\left(H^{\mathbb{C}} / P\right)\right)$ such that $\varphi \in H^{0}\left(E\left(\mathfrak{m}^{\mathbb{C}}\right)_{\sigma, \chi}^{-} \otimes K\right)$, we have

$$
\operatorname{deg}(E)(\sigma, \chi) \geq 0
$$

The pair $(E, \varphi)$ is called stable if it is semistable and furthermore: for any $P, \chi$ and $\sigma$ as above, such that $\varphi \in H^{0}\left(E\left(\mathfrak{m}^{\mathbb{C}}\right)_{\sigma, \chi}^{-} \otimes K\right)$ and such that $P \neq H^{\mathbb{C}}$, we have

$$
\operatorname{deg}(E)(\sigma, \chi)>0 .
$$

Finally, $(E, \varphi)$ is called polystable if it is semistable and for any $P, \chi$ and $\sigma$ as above, such that $\varphi \in H^{0}\left(E\left(\mathfrak{m}^{\mathbb{C}}\right)_{\sigma, \chi}^{-} \otimes K\right), P \neq H^{\mathbb{C}}$ and $\chi$ is strictly antidominant, and such that

$$
\operatorname{deg}(E)(\sigma, \chi)=0
$$

there is a holomorphic reduction of the structure group $\sigma_{L} \in \Gamma\left(E_{\sigma}(P / L)\right)$. Furthermore, under these hypothesis $\varphi$ is required to belong to $H^{0}\left(E\left(\mathfrak{m}^{\mathbb{C}}\right)_{\sigma_{L}, \chi}^{0} \otimes K\right)$.

These notions of semistability, stability and polystability can be generalized to $G$ Higgs bundle with $H^{\mathbb{C}}$ reductive but not semisimple. In this case, the notions depend on a parameter $\alpha \in Z\left(\mathfrak{h}^{\mathbb{C}}\right)$ which is equal to zero when $H^{\mathbb{C}}$ is semisimple (see [19]).

Definition 1.3. The moduli space of polystable G-Higgs bundles is defined as the set of isomorphisms classes of polystable $G$-Higgs bundles and is denoted by $\mathcal{M}(G)$.

\subsection{Stability in terms of filtrations}

Let $(E, \varphi)$ be a $G$-Higgs bundle such that $H^{\mathbb{C}}$ is a semisimple classical complex Lie group, and consider a parabolic subgroup $P \subseteq H^{\mathbb{C}}$ and its Levi subgroup $L \subseteq P$.

A workable notion of semistability, stability and polystability can be obtained giving a description of the objects involved in Definition 1.2 in terms of filtrations of vector bundles (see [19, Section 2.8]). 
Since $H^{\mathbb{C}}$ is a classical group, we have an inclusion $H^{\mathbb{C}} \subseteq \operatorname{GL}(n, \mathbb{C})$ for some $n$, and using the standard representation $\rho: H^{\mathbb{C}} \rightarrow \mathrm{GL}(n, \mathbb{C})$, we can associate to $E$ a holomorphic vector bundle $\mathbb{E}=E \times{ }_{\rho} \mathbb{C}^{n}$.

If $\chi$ is an antidominant character for $P, d \rho\left(s_{\chi}\right)$ diagonalizes as an element in $\mathfrak{g l}(n, \mathbb{C})$ with real eigenvalues $\lambda_{1}<\cdots<\lambda_{k}$ and this gives rise to a decomposition $\mathbb{E}=\bigoplus_{j=1}^{k} E\left(\lambda_{j}\right)$, where $E\left(\lambda_{j}\right)=\operatorname{ker}\left(\lambda_{j}-d \rho s_{\chi}\right)$. For each $i$ consider the holomorphic subbundle $E_{i}=$ $\bigoplus_{j \leq i} E\left(\lambda_{j}\right) \subset \mathbb{E}$. This induces a filtration

$$
\mathcal{E}=\left(0 \subset E_{1} \subset \cdots \subset E_{k}=\mathbb{E}\right)
$$

That is, to have a reduction of the structure group from $H^{\mathbb{C}}$ to the parabolic is the same as to have a filtration of this form. The parabolic subgroup $P_{s_{\chi}}$ is precisely the subgroup which preserve this filtration, that is,

$$
P_{s_{\chi}}=\left\{g \in H^{\mathbb{C}} \mid g\left(E_{i}\right) \subset E_{i} \text { for any } i\right\} .
$$

In these terms, the vector bundles $E\left(\mathfrak{m}^{\mathbb{C}}\right)_{\sigma, \chi}^{-}$and $E\left(\mathfrak{m}^{\mathbb{C}}\right)_{\sigma, \chi}^{0}$ can be expressed as

$$
\begin{aligned}
& E\left(\mathfrak{m}^{\mathbb{C}}\right)_{\sigma, \chi}^{-}=E\left(\mathfrak{m}^{\mathbb{C}}\right) \cap\left(\left(E^{p_{1}, q_{1}}\right)_{\sigma, \chi}^{-} \oplus \cdots \oplus\left(E^{p_{r}, q_{r}}\right)_{\sigma, \chi}^{-}\right), \\
& E\left(\mathfrak{m}^{\mathbb{C}}\right)_{\sigma, \chi}^{0}=E\left(\mathfrak{m}^{\mathbb{C}}\right) \cap\left(\left(E^{p_{1}, q_{1}}\right)_{\sigma, \chi}^{0} \oplus \cdots \oplus\left(E^{p_{r}, q_{r}}\right)_{\sigma, \chi}^{0}\right),
\end{aligned}
$$

where we are using the notation $E^{p, q}=E^{\otimes p} \otimes\left(E^{*}\right)^{\otimes q}$, and with

$$
\begin{aligned}
& \left(E^{p, q}\right)_{\sigma, \chi}^{-}=\sum_{\lambda_{i_{1}}+\cdots+\lambda_{i_{p}} \leq \lambda_{j_{1}}+\cdots+\lambda_{j_{q}}} E_{i_{1}} \otimes \cdots \otimes E_{i_{p}} \otimes E_{j_{1}}^{\perp} \otimes \cdots \otimes E_{j_{q}}^{\perp} \subset E^{p, q}, \\
& \left(E^{p, q}\right)_{\sigma, \chi}^{0}=\sum_{\lambda_{i_{1}}+\cdots+\lambda_{i_{p}}=\lambda_{j_{1}}+\cdots+\lambda_{j_{q}}} E_{i_{1}} \otimes \cdots \otimes E_{i_{p}} \otimes E_{j_{1}}^{\perp} \otimes \cdots \otimes E_{j_{q}}^{\perp} \subset E^{p, q},
\end{aligned}
$$

where $E_{j}^{\perp}=\left\{e \in E^{*} \mid\left\langle e, E_{j}\right\rangle=0\right\}$ and $\langle$,$\rangle is the natural pairing between E$ and $E^{*}$.

Finally, the degree can be computed as

$$
\operatorname{deg}(E)(\sigma, \chi)=\lambda_{k} \operatorname{deg} \mathbb{E}+\sum_{i=1}^{k-1}\left(\lambda_{i}-\lambda_{i+1}\right) \operatorname{deg} E_{i}
$$





\section{$2 \mathrm{SO}(n, \mathbb{C})$-Higgs bundles}

In this section we apply Definition 1.1 and the notions of stability described in Chapter 1 to the case $G=\mathrm{SO}(n, \mathbb{C})$.

Definition 2.1. An $\mathrm{SO}(n, \mathbb{C})$-Higgs bundle is a pair $(E, \phi)$ where $E$ is a principal $\mathrm{SO}(n, \mathbb{C})$-bundle and $\phi \in H^{0}(E(\mathfrak{s o}(n, \mathbb{C})) \otimes K)$.

If $(E, \phi)$ is an $\mathrm{SO}(n, \mathbb{C})$-Higgs bundle, using the standard representations of $\mathrm{SO}(n, \mathbb{C})$ in $\mathbb{C}^{n}$ we can associate to the principal $\mathrm{SO}(n, \mathbb{C})$-bundle $E$ a holomorphic vector bundle $\mathbb{E}$ of rank $n$,

$$
\mathbb{E}=E \times_{\mathrm{SO}(n, \mathbb{C})} \mathbb{C}^{n}
$$

with trivial determinant, together with a non-degenerate symmetric quadratic form $Q$ : $\mathbb{E} \otimes \mathbb{E} \rightarrow \mathbb{C}$, that is, there is a one-to-one correspondence between principal $\operatorname{SO}(n, \mathbb{C})$ bundles and pairs $(\mathbb{E}, Q)$. Of course, if $\mathbb{E}$ is a vector bundle of rank $n$ and trivial determinant equipped with a non-degenerate symmetric quadratic form $Q$, then $Q$ induces an isomorphism $Q: \mathbb{E} \rightarrow \mathbb{E}^{*}$ and, taking determinants, an isomorphism $\operatorname{det} Q: \operatorname{det} \mathbb{E} \rightarrow$ $(\operatorname{det} \mathbb{E})^{*}$. This means that $\operatorname{det} Q$ gives an isomorphism $(\operatorname{det} \mathbb{E})^{2} \cong \mathcal{O}$. So, to give a principal $\mathrm{SO}(n, \mathbb{C})$-bundle it is necessary to give a triple $(\mathbb{E}, Q, q)$, where $\mathbb{E}$ is a holomorphic vector bundle of rank $n, Q$ is a non-degenerate symmetric quadratic form on $\mathbb{E}$ and $q: \operatorname{det} \mathbb{E} \cong \mathcal{O}$ is an isomorphism satisfying $q^{2}=\operatorname{det} Q$. We will omit $q$ in this thesis for simplicity, since it will not play a role in our analysis.

The Higgs field in terms of the vector bundle $\mathbb{E}$ is a section $\phi \in H^{0}(\mathfrak{s o}(\mathbb{E}) \otimes K)$, where

$$
\mathfrak{s o}(\mathbb{E})=\{f \in \operatorname{End}(\mathbb{E}) \mid Q(f \cdot, \cdot)+Q(\cdot, f \cdot)=0 \text { and } \operatorname{Tr}(f)=0\} .
$$

Hence, $\mathrm{SO}(n, \mathbb{C})$-Higgs bundles are in one-to-one correspondence with triples $(\mathbb{E}, Q, \phi)$.

\subsection{Parabolic subgroups of $\mathrm{SO}(n, \mathbb{C})$ and filtrations}

Let $n=2 m$ or $n=2 m+1$. Using the orthogonal form $Q=\left(\begin{array}{c}I_{m} \\ I_{m}\end{array}\right)$, or $Q=$ $\left(\begin{array}{cc|c} & I_{m} & \\ I_{m} & & \\ \hline & & 1\end{array}\right)$ in the odd case, to define the Lie algebra $\mathfrak{s o}(n, \mathbb{C})$, we can consider the Cartan subalgebra,

$$
\mathfrak{c}=\{\text { diagonal matrices in } \mathfrak{s o}(n, \mathbb{C})\}=\left\langle C_{i}=E_{i i}-E_{n+i, n+i}, i=1, \ldots, m\right\rangle,
$$


for $n=2 m$ or $n=2 m+1$. The sets of $\operatorname{roots}$ of $\mathfrak{s o}(n, \mathbb{C})$ with respect to this Cartan subalgebra are

$$
\begin{aligned}
& \Delta=\left\{ \pm e_{i} \pm e_{j} \text { with } i \neq j\right\} \subset \mathfrak{c}^{*}, \text { for } n \text { even, } \\
& \Delta=\left\{ \pm e_{i} \pm e_{j} \text { with } i \neq j\right\} \cup\left\{ \pm e_{k}\right\} \subset \mathfrak{c}^{*}, \text { for } n \text { odd, }
\end{aligned}
$$

where $e_{i}\left(\begin{array}{ccc}c_{1} & & \\ & \ddots & \\ & & c_{n}\end{array}\right)=c_{i}$, and the sets of simple roots are

$$
\begin{aligned}
& \Pi=\left\{\alpha_{i}=e_{i}-e_{i+1}(1 \leq i \leq m-1), \alpha_{m}=e_{m-1}+e_{m}\right\}, \text { for } n=2 m \text { even, } \\
& \Pi=\left\{\alpha_{i}=e_{i}-e_{i+1}(1 \leq i \leq m-1), \alpha_{m}=e_{m}\right\}, \text { for } n=2 m+1 \text { odd }
\end{aligned}
$$

For any subset $A \subset \Pi$ we can define a parabolic subgroup $P_{A} \subset \mathrm{SO}(n, \mathbb{C})$ and a Levi subgroup $L_{A} \subset P_{A}$ as in Section 1.1 .

An antidominant character for a parabolic subgroup $P_{A}$ is in this case an element of the form

$$
\chi=\sum_{\alpha_{i} \in A} m_{i} \lambda_{i}
$$

with all $m_{i} \leq 0$, where the fundamental weights are:

$$
\begin{aligned}
\lambda_{i}= & \alpha_{1}+2 \alpha_{2}+\ldots+(i-1) \alpha_{i-1}+i\left(\alpha_{i}+\ldots+\alpha_{m-2}\right)+\frac{1}{2} i\left(\alpha_{m-1}+\alpha_{m}\right), \\
& \text { for } i<m-1 \\
\lambda_{m-1}= & \frac{1}{2}\left(\alpha_{1}+2 \alpha_{2}+\ldots+(m-2) \alpha_{m-2}+\frac{1}{2} m \alpha_{m-1}+\frac{1}{2}(m-2) \alpha_{m}\right), \\
\lambda_{m}= & \frac{1}{2}\left(\alpha_{1}+2 \alpha_{2}+\ldots+(m-2) \alpha_{m-2}+\frac{1}{2}(m-2) \alpha_{m-1}+\frac{1}{2} m \alpha_{m}\right),
\end{aligned}
$$

for $n=2 m$ even, and

$$
\begin{aligned}
\lambda_{i} & =\alpha_{1}+2 \alpha_{2}+\ldots+(i-1) \alpha_{i-1}+i\left(\alpha_{i}+\ldots+\alpha_{m}\right) \text { for } i<m, \\
\lambda_{m} & =\frac{1}{2}\left(\alpha_{1}+2 \alpha_{2}+\ldots+m \alpha_{m}\right)
\end{aligned}
$$

for $n=2 m+1$ odd.

Let $E$ be a principal $\mathrm{SO}(n, \mathbb{C})$-bundle and consider a reduction of the structure group of $E$ to a parabolic subgroup $P$ of $\mathrm{SO}(n, \mathbb{C})$. Let $\mathbb{E}$ be the holomorphic vector bundle associated to $E$ via the standard representation and let $s=i X \in i \mathfrak{s o}(n)$ be the element associated to an antidominant character for $P$ as in the previous section.

The eigenvalues of a skew-symmetric matrix always come in pairs $\pm \mu_{j}$ (except in the odd-dimensional case where there is an additional unpaired 0 eigenvalue). For a real 
skew-symmetric matrix the non-zero eigenvalues are all pure imaginary and thus are of the form $\pm i \lambda_{j}$, with $\lambda_{j} \in \mathbb{R}$. Any real skew-symmetric matrix can be diagonalized by a unitary matrix. Since the eigenvalues of a real skew-symmetric matrix are complex it is not possible to diagonalize by a real matrix, however, it is possible to bring every skew-symmetric matrix to a block diagonal form

$$
\left(\begin{array}{cccccccccc}
0 & \lambda_{1} & & & & & & & & \\
-\lambda_{1} & 0 & & & & & & & & \\
& & 0 & \lambda_{2} & & & & & & \\
& & -\lambda_{2} & 0 & & & & & & \\
& & & & \ddots & & & & & \\
& & & & & -\lambda_{l} & 0 & & & \\
& & & & & & & 0 & & \\
& & & & & & & & \ddots & 0
\end{array}\right)
$$

by an orthogonal transformation. If $\left\{v_{1}, v_{1}^{\prime}, \ldots, v_{l}, v_{l}^{\prime}, v_{2 l+1}, \ldots, v_{m}\right\}$ is the orthogonal basis in which $X$ has block diagonal form, then $\left\{v_{1}+i v_{1}^{\prime}, \ldots, v_{l}+i v_{l}^{\prime}, v_{2 l+1}, \ldots, v_{m}, i v_{l}+\right.$ $\left.v_{l}^{\prime}, \ldots, i v_{1}+v_{1}^{\prime}\right\}$, is the basis in which $s$ takes form

$$
\left(\begin{array}{ccccccccc}
-\lambda_{1} & & & & & & & & \\
& \ddots & & & & & & & \\
& & -\lambda_{l} & & & & & & \\
& & & 0 & & & & & \\
& & & & \ddots & & & & \\
& & & & & & \lambda_{l} & & \\
& & & & & & & \ddots & \\
& & & & & & & \lambda_{1}
\end{array}\right) .
$$

Using this diagonal form, if $g=\left(g_{i j}\right) \in \mathrm{SO}(n, \mathbb{C})$, then we have

$$
\begin{gathered}
\operatorname{Ad}\left(e^{t s}\right)(g)=e^{t s} g e^{-t s}=\operatorname{Diag}\left(e^{-t \lambda_{1}}, \ldots, e^{-t \lambda_{l}}, 1, \ldots, 1, e^{t \lambda_{l}}, \ldots, e^{t \lambda_{1}}\right) \cdot\left(g_{i j}\right) \\
\cdot \operatorname{Diag}\left(e^{t \lambda_{1}}, \ldots, e^{t \lambda_{l}}, 1, \ldots, 1, e^{-t \lambda_{l}}, \ldots, e^{-t \lambda_{1}}\right) .
\end{gathered}
$$

If all the eigenvalues of $s$ are different, $P$ is the subgroup of upper triangular matrices in $\mathrm{SO}(n, \mathbb{C})$ and if they are not, we have block upper diagonal matrices. Considering the sequence of different eigenvalues of $s, \lambda_{1}<\lambda_{2}<\ldots<\lambda_{k-1}<\lambda_{k}$, with $\lambda_{k-i+1}+\lambda_{i}=0$, we can construct a filtration

$$
\mathcal{E}=\left(0 \subset E_{1} \subset \cdots \subset E_{k}=\mathbb{E}\right)
$$

as in Section 1.3. In this case, since $\lambda_{k-i+1}+\lambda_{i}=0$, the subbundles in the filtration satisfy $E_{j}=E_{k-j}^{\perp_{Q}}$, that is, $\mathcal{E}$ is a filtration of isotropic subbundles.

In the following example we study the parabolic subgroups of $\operatorname{SO}(6, \mathbb{C})$, which is a simple but rich enough case to illustrate this theory. 
Example 2.2. Parabolic subgroups of $\mathrm{SO}(6, \mathbb{C})$.

Every parabolic subgroup of $\mathrm{SO}(6, \mathbb{C})$ is in correspondence with an element $s \in i \mathfrak{s o}(6)$, that is, the parabolic subgroups of $\mathrm{SO}(6, \mathbb{C})$ are defined by

$$
P_{s}=\left\{g \in \mathrm{SO}(6, \mathbb{C}) \mid e^{t s} g e^{-t s} \text { is bounded as } t \rightarrow \infty\right\},
$$

where $s$ takes all the possible values in $i \mathfrak{s o}(6)$. The corresponding Levi subgroups are defined by

$$
L_{s}=\{g \in \mathrm{SO}(6, \mathbb{C}) \mid \operatorname{Ad}(g)(s)=s\}
$$

Let $-\lambda_{1} \leq-\lambda_{2} \leq-\lambda_{3} \leq \lambda_{3} \leq \lambda_{2} \leq \lambda_{1}$ be the eigenvalues of $s$. If $g=\left(g_{i j}\right) \in \operatorname{SO}(6, \mathbb{C})$, then we have that $e^{t s} g e^{-t s}$ is equal to

$$
\left(\begin{array}{cccccc}
g_{11} & e^{t\left(\lambda_{2}-\lambda_{1}\right)} g_{12} & e^{t\left(\lambda_{3}-\lambda_{1}\right)} g_{13} & e^{-t\left(\lambda_{1}+\lambda_{3}\right)} g_{14} & e^{-t\left(\lambda_{1}+\lambda_{2}\right)} g_{15} & e^{-2 t \lambda_{1}} g_{16} \\
e^{t\left(\lambda_{1}-\lambda_{2}\right)} g_{21} & g_{22} & e^{t\left(\lambda_{3}-\lambda_{2}\right)} g_{23} & e^{-t\left(\lambda_{2}+\lambda_{3}\right)} g_{24} & e^{-2 t \lambda_{2}} g_{25} & e^{-t\left(\lambda_{1}+\lambda_{2}\right)} g_{26} \\
e^{t\left(\lambda_{1}-\lambda_{3}\right)} g_{31} & e^{t\left(\lambda_{2}-\lambda_{3}\right)} g_{32} & g_{33} & e^{-2 t \lambda_{3}} g_{34} & e^{-t\left(\lambda_{2}+\lambda_{3}\right)} g_{35} & e^{-t\left(\lambda_{1}+\lambda_{3}\right)} g_{36} \\
e^{t\left(\lambda_{1}+\lambda_{3}\right)} g_{41} & e^{t\left(\lambda_{2}+\lambda_{3}\right)} g_{42} & e^{2 t \lambda_{3}} g_{43} & g_{44} & e^{t\left(\lambda_{3}-\lambda_{2}\right)} g_{45} & e^{t\left(\lambda_{3}-\lambda_{1}\right)} g_{46} \\
e^{t\left(\lambda_{1}+\lambda_{2}\right)} g_{51} & e^{2 t \lambda_{2}} g_{52} & e^{t\left(\lambda_{2}+\lambda_{3}\right)} g_{53} & e^{t\left(\lambda_{2}-\lambda_{3}\right)} g_{54} & g_{55} & e^{t\left(\lambda_{2}-\lambda_{1}\right)} g_{56} \\
e^{2 t \lambda_{1}} g_{61} & e^{t\left(\lambda_{1}+\lambda_{2}\right)} g_{62} & e^{t\left(\lambda_{1}+\lambda_{3}\right)} g_{63} & e^{t\left(\lambda_{1}-\lambda_{3}\right)} g_{64} & e^{t\left(\lambda_{1}-\lambda_{2}\right)} g_{65} & g_{66}
\end{array}\right) \text {. }
$$

Studying the behavior of this matrix when $t \rightarrow \infty$ for all possible values of $\lambda_{i}$ we obtain the list of parabolic subgroups of $\mathrm{SO}(6, \mathbb{C})$ :

$$
\begin{aligned}
& P=\operatorname{SO}(6, \mathbb{C}), \quad P_{1}=\left(\begin{array}{llllll}
* & * & * & * & * & * \\
* & * & * & * & * & * \\
* & * & * & * & * & * \\
& & & * & * & * \\
& & * & * & * \\
& & & * & * & *
\end{array}\right), \quad P_{2}=\left(\begin{array}{llllll}
* & * & * & * & * & * \\
* & * & * & * & * \\
* & * & * & * & * \\
* & * & * & * & * \\
* & * & * & * & * \\
& & & & & *
\end{array}\right), \\
& P_{3}=\left(\begin{array}{llllll}
* & * & * & * & * & * \\
* & * & * & * & * & * \\
& & * & * & * & * \\
& & * & * & * & * \\
& & & * & * \\
& & & * & *
\end{array}\right), \quad P_{4}=\left(\begin{array}{llllll}
* & * & * & * & * & * \\
& * & * & * & * & * \\
& * & * & * & * & * \\
& & * & * & * \\
& & * & * & * \\
& & & & *
\end{array}\right), \quad P_{5}=\left(\begin{array}{llllll}
* & * & * & * & * & * \\
* & * & * & * & * & * \\
& & * & * & * & * \\
& & * & * & * \\
& & & * & * \\
& & & & * & *
\end{array}\right), \\
& P_{6}=\left(\begin{array}{llllll}
* & * & * & * & * & * \\
& * & * & * & * & * \\
& & * & * & * & * \\
& & * & * & * & * \\
& & & * & * \\
& & & & *
\end{array}\right), \quad P_{7}=\left(\begin{array}{llllll}
* & * & * & * & * & * \\
& * & * & * & * & * \\
& & * & * & * & * \\
& & & * & * & * \\
& & & & * & * \\
& & & & & *
\end{array}\right) \text {. }
\end{aligned}
$$

Observe that $P_{i}$ are not matrices in $\operatorname{SL}(6, \mathbb{C})$ but in $\mathrm{SO}(6, \mathbb{C})$, this means that there are relations between the elements in a position marked by $*$ and some of them are in fact zero. 
The corresponding Levi subgroups are

$$
\begin{aligned}
& L=\mathrm{SO}(6, \mathbb{C}), \quad L_{1}=\left(\begin{array}{llllll}
* & * & * & & & \\
* & * & * & & & \\
* & * & * & & & \\
& & & * & * & * \\
& & & * & * & * \\
& & & * & * & *
\end{array}\right), \quad L_{2}=\left(\begin{array}{llllll}
* & & & & & \\
& * & * & * & * & \\
* & * & * & * \\
* & * & * & * \\
* & * & * & * \\
& & & & & *
\end{array}\right),
\end{aligned}
$$

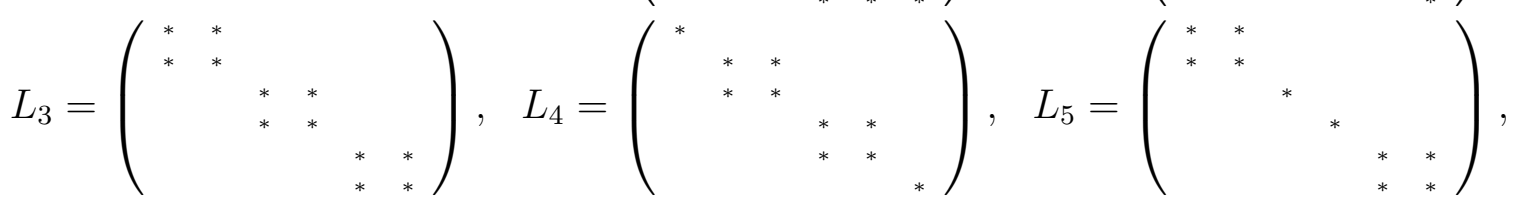

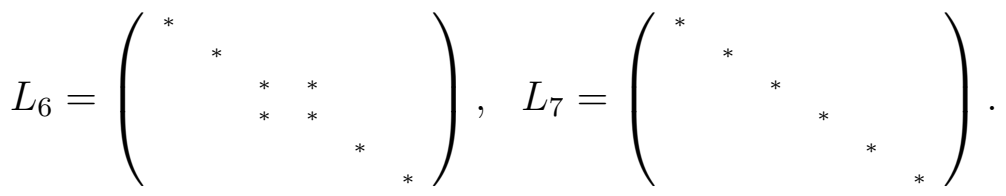

Let $E$ be a principal $\mathrm{SO}(6, \mathbb{C})$-bundle $E$. A reduction of the structure group of $E$ to a parabolic subgroup $P_{i}$ is equivalent to the existence of a filtration of its associated vector bundle $\mathbb{E}$. To conclude with this example, we describe the filtration corresponding to each parabolic subgroup $P_{i}$ of $\mathrm{SO}(6, \mathbb{C})$ :

$$
\begin{array}{llr}
P: & \mathcal{E}=(0 \subset \mathbb{E}), & \lambda_{i}=0, \\
P_{1}: & \mathcal{E}=\left(0 \subset E_{1}^{(3)} \subset \mathbb{E}\right), & -\lambda=-\lambda=-\lambda<\lambda=\lambda=\lambda, \\
P_{2}: & \mathcal{E}=\left(0 \subset E_{1}^{(1)} \subset E_{2}^{(5)} \subset \mathbb{E}\right), & -\lambda<0=0=0=0<\lambda, \\
P_{3}: & \mathcal{E}=\left(0 \subset E_{1}^{(2)} \subset E_{2}^{(4)} \subset \mathbb{E}\right), & -\lambda=-\lambda<0=0<\lambda=\lambda, \\
P_{4}: & \mathcal{E}=\left(0 \subset E_{1}^{(1)} \subset E_{2}^{(3)} \subset E_{3}^{(5)} \subset \mathbb{E}\right), & -\lambda<-\mu=-\mu<\mu=\mu<\lambda, \\
P_{5}: & \mathcal{E}=\left(0 \subset E_{1}^{(2)} \subset E_{2}^{(3)} \subset E_{3}^{(4)} \subset \mathbb{E}\right), & -\lambda=-\lambda<-\mu<\mu<\lambda=\lambda, \\
P_{6}: & \mathcal{E}=\left(0 \subset E_{1}^{(1)} \subset E_{2}^{(2)} \subset E_{3}^{(4)} \subset E_{4}^{(5)} \subset \mathbb{E}\right), & -\lambda<-\mu<0=0<\mu<\lambda, \\
P_{7}: \mathcal{E}=\left(0 \subset E_{1}^{(1)} \subset E_{2}^{(2)} \subset E_{3}^{(3)} \subset E_{4}^{(4)} \subset E_{5}^{(5)} \subset \mathbb{E}\right), & -\lambda<-\mu<-\gamma<\gamma<\mu<\lambda .
\end{array}
$$

In the filtrations, we have the relation $E_{j} \cong E_{k-j}^{\perp_{Q}}$ and the number $(i)$ denotes the dimension of each subbundle.

\subsection{Stability of $\mathrm{SO}(n, \mathbb{C})$-Higgs bundles}

Applying Definition 1.2 to $\mathrm{SO}(n, \mathbb{C})$-Higgs bundles and using the results of Section 1.3 we obtain the following:

Proposition 2.3. An $\mathrm{SO}(n, \mathbb{C})$-Higgs bundle $(\mathbb{E}, Q, \phi)$ with $n \neq 2$ is semistable if for any filtration

$$
\mathcal{E}=\left(0 \subset E_{1} \subset \ldots \subset E_{k}=\mathbb{E}\right),
$$

$1 \leq k \leq n$, satisfying $E_{j}=E_{k-j}^{\perp_{Q}}$, and any element of

$$
\Lambda(\mathcal{E})=\left\{\lambda=\left(\lambda_{1} \leq \lambda_{2} \leq \ldots \leq \lambda_{k}\right) \in \mathbb{R}^{k} \mid \lambda_{k-i+1}+\lambda_{i}=0 \text { for any } i\right\}
$$


such that $\phi \in H^{0}(N(\mathcal{E}, \lambda) \otimes K)$, where

$$
N(\mathcal{E}, \lambda)=\mathfrak{s o}(\mathbb{E}) \cap \sum_{\lambda_{j} \leq \lambda_{i}} \operatorname{Hom}\left(E_{i}, E_{j}\right),
$$

we have

$$
d(\mathcal{E}, \lambda)=\sum_{j=1}^{k-1}\left(\lambda_{j}-\lambda_{j+1}\right) \operatorname{deg} E_{j} \geq 0 .
$$

The triple $(\mathbb{E}, Q, \phi)$ is stable if it is semistable and for any choice of the filtration $\mathcal{E}$ and non-zero $\lambda \in \Lambda(\mathcal{E})$ such that $\phi \in H^{0}(N(\mathcal{E}, \lambda) \otimes K)$, we have

$$
d(\mathcal{E}, \lambda)>0
$$

Finally, the triple $(\mathbb{E}, Q, \phi)$ is polystable if it is semistable and for any filtration $\mathcal{E}$ as above and $\lambda \in \Lambda(\mathcal{E})$ satisfying $\lambda_{i}<\lambda_{i+1}$ for each $i, \phi \in H^{0}(N(\mathcal{E}, \lambda) \otimes K)$ and $d(\mathcal{E}, \lambda)=0$, there is an isomorphism

$$
\mathbb{E} \simeq E_{1} \oplus E_{2} / E_{1} \oplus \cdots \oplus E_{k} / E_{k-1}
$$

satisfying

$$
Q\left(E_{i} / E_{i-1}, E_{j} / E_{j-1}\right)=0 \text { unless } i+j=k+1 .
$$

Furthermore, via the isomorphism,

$$
\phi \in H^{0}\left(\bigoplus_{i} \operatorname{Hom}\left(E_{i} / E_{i-1}, E_{i} / E_{i-1}\right) \otimes K\right)
$$

There is a simplification of the semistability and stability conditions, which is next described.

Proposition 2.4. An $\mathrm{SO}(n, \mathbb{C})$-Higgs bundle $(\mathbb{E}, Q, \phi)$ with $n \neq 2$ issemistable if and only if for any isotropic subbundle $E^{\prime} \subset \mathbb{E}$ such that $\phi\left(E^{\prime}\right) \subseteq E^{\prime} \otimes K$ the inequality $\operatorname{deg} E^{\prime} \leq 0$ holds, and it is stable if it is semistable and for any non-zero isotropic subbundle $E^{\prime} \subset \mathbb{E}$ such that $\phi\left(E^{\prime}\right) \subseteq E^{\prime} \otimes K$ we have $\operatorname{deg} E^{\prime}<0$.

Proof. This proof is analogous to the proof of Theorem 3.9 in [19].

Let $(\mathbb{E}, Q, \phi)$ be an $\mathrm{SO}(n, \mathbb{C})$-Higgs bundle and assume that for any isotropic subbundle $E^{\prime} \subset \mathbb{E}$ such that $\phi\left(E^{\prime}\right) \subseteq E^{\prime} \otimes K$ one has $\operatorname{deg} E^{\prime} \leq 0$. We are going to prove that $(\mathbb{E}, Q, \phi)$ is semistable.

Choose any filtration $\mathcal{E}=\left(0 \subset E_{1} \subset \ldots \subset E_{k}=\mathbb{E}\right)$ satisfying $E_{j}=E_{k-j}^{\perp_{Q}}$ for any $j$. We have to understand the geometry of the set

$$
\Lambda(\mathcal{E}, \phi)=\{\lambda \in \Lambda(\mathcal{E}) \mid \phi \in N(\mathcal{E}, \lambda)\} \subset \mathbb{R}^{k}
$$


Let $\mathcal{J}=\left\{j \mid \phi\left(E_{j}\right) \subseteq E_{j} \otimes K\right\}=\left\{j_{1}, \ldots, j_{r}\right\}$. One checks easily that if $\lambda=\left(\lambda_{1}, \ldots, \lambda_{k}\right) \in$ $\Lambda(\mathcal{E})$ then

$$
\lambda \in \Lambda(\mathcal{E}, \phi) \Leftrightarrow \lambda_{a}=\lambda_{b} \text { for any } j_{i} \leq a \leq b \leq j_{i+1} .
$$

The set of indices $\mathcal{J}$ is symmetric:

$$
j \in \mathcal{J} \Leftrightarrow k-j \in \mathcal{J}
$$

To check this we have to prove that $\phi\left(E_{j}\right) \subseteq E_{j} \otimes K$ implies that $\phi\left(E_{j}^{\perp_{Q}}\right) \subseteq E_{j}^{\perp_{Q}} \otimes K$. Suppose that this is not true, then there is a $j$ with $\phi\left(E_{j}\right) \subseteq E_{j} \otimes K$ and there exists some $w \in E_{j}^{\perp_{Q}}$ such that $\phi(w) \notin E_{j}^{\perp_{Q}} \otimes K$. Then there exists $v \in E_{j}$ such that $Q(v, \phi(w)) \neq 0$. However, since $\phi \in H^{0}(\mathfrak{s o}(\mathbb{E}) \otimes K)$, we must have

$$
Q(v, \phi(w))=Q\left(v,-\phi^{\top}(w)\right)=-Q(\phi(v), w)
$$

and the latter vanishes because by assumption $\phi(v)$ belongs to $E_{j}$. So we have reached a contradiction.

Let $\mathcal{J}^{\prime}=\{j \in \mathcal{J} \mid 2 j \leq k\}$ and define for any $j \in \mathcal{J}^{\prime}$ the vector

$$
L_{j}=-\sum_{c \leq j} e_{c}+\sum_{d \geq k-j+1} e_{d}
$$

where $e_{1}, \ldots, e_{k}$ is the canonical basis of $\mathbb{R}^{k}$. We know that the set $\Lambda(\mathcal{E}, \phi)$ is the positive span of the vectors $\left\{L_{j} \mid j \in \mathcal{J}^{\prime}\right\}$. Consequently, we have

$$
d(\mathcal{E}, \lambda) \geq 0 \text { for any } \lambda \in \Lambda(\mathcal{E}, \phi) \Leftrightarrow d\left(\mathcal{E}, L_{j}\right) \geq 0 \text { for any } j
$$

and $d\left(\mathcal{E}, L_{j}\right)=-\operatorname{deg} E_{k-j}-\operatorname{deg} E_{j}$. Since $\operatorname{deg} E_{k-j}=\operatorname{deg} E_{j}, d\left(\mathcal{E}, L_{j}\right) \geq 0$ is equivalent to $\operatorname{deg} E_{j} \leq 0$, which holds by assumption. Hence $(\mathbb{E}, Q, \phi)$ is semistable.

Conversely, if $(\mathbb{E}, Q, \phi)$ is semistable then for any isotropic subbundle $E^{\prime} \subset \mathbb{E}$ such that $\phi\left(E^{\prime}\right) \subseteq E^{\prime} \otimes K$ we have $\operatorname{deg} E^{\prime} \leq 0$ is immediate by applying the semistability condition of the filtration $0 \subset E^{\prime} \subset E^{\prime \perp_{Q}} \subset \mathbb{E}$.

Finally, the proof of the second statement on stability is very similar to the case of semistability, so we omit it.

The case $n=2$ requires a special attention. Observe that a principal $\mathrm{SO}(2, \mathbb{C})$-bundle $(E, Q)$ decomposes as $E=L \oplus L^{-1}$, where $L$ is a line bundle and $Q=\left(\begin{array}{c}1 \\ 1\end{array}\right)$. Then, any principal $\mathrm{SO}(2, \mathbb{C})$-bundle has an isotropic subbundle with degree greater or equal than zero. However, from the isomorphism $\mathrm{SO}(2, \mathbb{C}) \cong \mathbb{C}^{*}$ we deduce that there are 
no proper parabolic subgroups in $\mathrm{SO}(2, \mathbb{C})$, and using Definition 1.2 we have that any $\mathrm{SO}(2, \mathbb{C})$-Higgs bundle is stable. Then, the simplified notions described in Definition 2.4 can not be applied to this case.

We illustrate Proposition 2.3 and Proposition 2.4 with the following example.

Example 2.5. Stability of $\mathrm{SO}(6, \mathbb{C})$-Higgs bundles.

Let $(\mathbb{E}, Q, \phi)$ be an $\mathrm{SO}(6, \mathbb{C})$-Higgs bundle. In this example we apply Theorem 2.3 to obtain stability conditions of $(\mathbb{E}, Q, \phi)$ for each parabolic subgroup of $\mathrm{SO}(6, \mathbb{C})$ and we arrive at the simplified notions of Theorem 2.4.

Let $P \subset \mathrm{SO}(n, \mathbb{C})$ be a parabolic subgroup and let $\chi$ be an strictly antidominant character for $P$. The fibre of the bundle $E\left(\mathfrak{m}^{\mathbb{C}}\right)_{\sigma, \chi}^{-}$of Definition 1.2 , which is denoted by $N(\mathcal{E}, \lambda)$ in Proposition 2.3 , is

$$
\left(\mathfrak{m}^{\mathbb{C}}\right)_{\chi}^{-}=\left\{v \in \mathfrak{m}^{\mathbb{C}} \mid \iota\left(e^{t s_{\chi}}\right) v \text { remains bounded as } t \rightarrow \infty\right\} .
$$

Since in the case of $\mathrm{SO}(6, \mathbb{C})$ the isotropy representation coincides with the adjoint representation, the condition $\phi \in H^{0}(N(\mathcal{E}, \lambda) \otimes K)$ is equivalent to

$$
\phi \in\left\{v \in \mathfrak{s o}(6, \mathbb{C}) \mid \operatorname{Ad}\left(e^{t s}\right) v \text { is bounded as } t \rightarrow \infty\right\} \cong \mathfrak{p} .
$$

If $v=\left(v_{i j}\right) \in \mathfrak{s o}(6, \mathbb{C})$, then we have that $\operatorname{Ad}\left(e^{t s}\right) v$ is equal to

$$
\left(\begin{array}{cccccc}
v_{11} & e^{t\left(\lambda_{2}-\lambda_{1}\right)} v_{12} & e^{t\left(\lambda_{3}-\lambda_{1}\right)} v_{13} & e^{-t\left(\lambda_{1}+\lambda_{3}\right)} v_{14} & e^{-t\left(\lambda_{1}+\lambda_{2}\right)} v_{15} & 0 \\
e^{t\left(\lambda_{1}-\lambda_{2}\right)} v_{21} & v_{22} & e^{t\left(\lambda_{3}-\lambda_{2}\right)} v_{23} & e^{-t\left(\lambda_{2}+\lambda_{3}\right)} v_{24} & 0 & -e^{-t\left(\lambda_{1}+\lambda_{2}\right)} v_{15} \\
e^{t\left(\lambda_{1}-\lambda_{3}\right)} v_{31} & e^{t\left(\lambda_{2}-\lambda_{3}\right)} v_{32} & v_{33} & 0 & -e^{-t\left(\lambda_{2}+\lambda_{3}\right)} v_{24} & -e^{-t\left(\lambda_{1}+\lambda_{3}\right)} v_{14} \\
e^{t\left(\lambda_{1}+\lambda_{3}\right)} v_{41} & e^{t\left(\lambda_{2}+\lambda_{3}\right)} v_{42} & 0 & -v_{33} & -e^{t\left(\lambda_{3}-\lambda_{2}\right)} v_{23} & -e^{t\left(\lambda_{3}-\lambda_{1}\right)} v_{13} \\
e^{t\left(\lambda_{1}+\lambda_{2}\right)} v_{51} & 0 & -e^{t\left(\lambda_{2}+\lambda_{3}\right)} v_{42} & -e^{t\left(\lambda_{2}-\lambda_{3}\right)} v_{32} & -v_{22} & -e^{t\left(\lambda_{2}-\lambda_{1}\right)} v_{12} \\
0 & -e^{t\left(\lambda_{1}+\lambda_{2}\right)} v_{51} & -e^{t\left(\lambda_{1}+\lambda_{3}\right)} v_{41} & -e^{t\left(\lambda_{1}-\lambda_{3}\right)} v_{31} & -e^{t\left(\lambda_{1}-\lambda_{2}\right)} v_{21} & -v_{11}
\end{array}\right) .
$$

The stability conditions for each parabolic subgroup are the following:

- $P_{1}$ : A reduction of the structure group of the $\mathrm{SO}(6, \mathbb{C})$-Higgs bundle $(\mathbb{E}, Q)$ to the parabolic subgroup $P_{1}$ is equivalent to a filtration $\mathcal{E}=\left(0 \subset E_{1}^{(3)} \subset \mathbb{E}\right)$, the set of eigenvalues of the corresponding $s_{1} \in i \mathfrak{s o}(6)$ is given by $\Lambda(\mathcal{E})=\{(-\lambda, \lambda)\}$ and the condition $\phi \in H^{0}(N(\mathcal{E}, \lambda) \otimes K)$ is equivalent to

$$
\phi=\left(\begin{array}{cccccc}
f_{1,1} & f_{1,2} & f_{1,3} & f_{1,4} & f_{1,5} & 0 \\
f_{2,1} & f_{2,2} & f_{2,3} & f_{2,4} & 0 & -f_{1,5} \\
f_{3,1} & f_{3,2} & f_{3,3} & 0 & -f_{2,4} & -f_{1,4} \\
0 & 0 & 0 & -f_{3,3} & -f_{2,3} & -f_{1,3} \\
0 & 0 & 0 & -f_{3,2} & -f_{2,2} & -f_{1,2} \\
0 & 0 & 0 & -f_{3,1} & -f_{2,1} & -f_{1,1}
\end{array}\right)
$$


Then,

$$
d(\mathcal{E}, \lambda)=(-\lambda-\lambda) \operatorname{deg} E_{1}^{(3)}=-2 \lambda \operatorname{deg} E_{1}^{(3)} \geq 0
$$

and $d(\mathcal{E}, \lambda) \geq 0$ if and only if

$$
\operatorname{deg} E_{1}^{(3)} \leq 0
$$

- $P_{2}$ : A reduction to $P_{2}$ is equivalent to a filtration $\mathcal{E}=\left(0 \subset E_{1}^{(1)} \subset E_{2}^{(5)} \subset \mathbb{E}\right)$, the set of possible weights is $\Lambda(\mathcal{E})=\{(-\lambda, 0, \lambda)\}$ and the condition given by the Higgs field is

$$
\phi=\left(\begin{array}{cccccc}
f_{1,1} & f_{1,2} & f_{1,3} & f_{1,4} & f_{1,5} & 0 \\
0 & f_{2,2} & f_{2,3} & f_{2,4} & 0 & -f_{1,5} \\
0 & f_{3,2} & f_{3,3} & 0 & -f_{2,4} & -f_{1,4} \\
0 & f_{4,2} & 0 & -f_{3,3} & -f_{2,3} & -f_{1,3} \\
0 & 0 & -f_{4,2} & -f_{3,2} & -f_{2,2} & -f_{1,2} \\
0 & 0 & 0 & 0 & 0 & -f_{1,1}
\end{array}\right) .
$$

Then,

$$
d(\mathcal{E}, \lambda)=(-\lambda-0) \operatorname{deg} E_{1}^{(1)}+(0-\lambda) \operatorname{deg} E_{2}^{(5)}=-2 \lambda \operatorname{deg} E_{1}^{(1)} \geq 0
$$

if and only if

$$
\operatorname{deg} E_{1}^{(1)} \leq 0
$$

- $P_{3}$ : For the parabolic subgroup $P_{3}$ we have the filtration $\mathcal{E}=\left(0 \subset E_{1}^{(2)} \subset E_{2}^{(4)} \subset \mathbb{E}\right)$, the set of weights $\Lambda(\mathcal{E})=\{(-\lambda, 0, \lambda)\}$ and the condition

$$
\phi=\left(\begin{array}{cccccc}
f_{1,1} & f_{1,2} & f_{1,3} & f_{1,4} & f_{1,5} & 0 \\
f_{2,1} & f_{2,2} & f_{2,3} & f_{2,4} & 0 & -f_{1,5} \\
0 & 0 & f_{3,3} & 0 & -f_{2,4} & -f_{1,4} \\
0 & 0 & 0 & -f_{3,3} & -f_{2,3} & -f_{1,3} \\
0 & 0 & 0 & 0 & -f_{2,2} & -f_{1,2} \\
0 & 0 & 0 & 0 & -f_{2,1} & -f_{1,1}
\end{array}\right) .
$$

Then,

$$
d(\mathcal{E}, \lambda)=(-\lambda-0) \operatorname{deg} E_{1}^{(2)}+(0-\lambda) \operatorname{deg} E_{2}^{(4)}=-2 \lambda \operatorname{deg} E_{1}^{(2)} \geq 0
$$

if and only if

$$
\operatorname{deg} E_{1}^{(2)} \leq 0
$$

- $P_{4}$ : For the parabolic $P_{4}$ we have the filtration $\mathcal{E}=\left(0 \subset E_{1}^{(1)} \subset E_{2}^{(3)} \subset E_{3}^{(5)} \subset \mathbb{E}\right)$, 
the set of weights $\Lambda(\mathcal{E})=\{(-\lambda,-\mu, \mu, \lambda)\}$, the condition

$$
\phi=\left(\begin{array}{cccccc}
f_{1,1} & f_{1,2} & f_{1,3} & f_{1,4} & f_{1,5} & 0 \\
0 & f_{2,2} & f_{2,3} & f_{2,4} & 0 & -f_{1,5} \\
0 & f_{3,2} & f_{3,3} & 0 & -f_{2,4} & -f_{1,4} \\
0 & 0 & 0 & -f_{3,3} & -f_{2,3} & -f_{1,3} \\
0 & 0 & 0 & -f_{3,2} & -f_{2,2} & -f_{1,2} \\
0 & 0 & 0 & 0 & 0 & -f_{1,1}
\end{array}\right),
$$

and the semistability condition implies

$$
\begin{aligned}
d(\mathcal{E}, \lambda) & =(-\lambda+\mu) \operatorname{deg} E_{1}^{(1)}-2 \mu \operatorname{deg} E_{2}^{(3)}+(\mu-\lambda) \operatorname{deg} E_{3}^{(5)} \\
& =-2(\lambda-\mu) \operatorname{deg} E_{1}^{(1)}-2 \mu \operatorname{deg} E_{2}^{(3)} \geq 0 .
\end{aligned}
$$

- $P_{5}$ : For this parabolic we have the filtration $\mathcal{E}=\left(0 \subset E_{1}^{(2)} \subset E_{2}^{(3)} \subset E_{3}^{(4)} \subset \mathbb{E}\right)$, the set of weights $\Lambda(\mathcal{E})=\{(-\lambda,-\mu, \mu, \lambda)\}$, the condition

$$
\phi=\left(\begin{array}{cccccc}
f_{1,1} & f_{1,2} & f_{1,3} & f_{1,4} & f_{1,5} & 0 \\
f_{2,1} & f_{2,2} & f_{2,3} & f_{2,4} & 0 & -f_{1,5} \\
0 & 0 & f_{3,3} & 0 & -f_{2,4} & -f_{1,4} \\
0 & 0 & 0 & -f_{3,3} & -f_{2,3} & -f_{1,3} \\
0 & 0 & 0 & 0 & -f_{2,2} & -f_{1,2} \\
0 & 0 & 0 & 0 & -f_{2,1} & -f_{1,1}
\end{array}\right),
$$

and the semistability condition implies

$$
\begin{aligned}
d(\mathcal{E}, \lambda) & =(-\lambda+\mu) \operatorname{deg} E_{1}^{(2)}-2 \mu \operatorname{deg} E_{2}^{(3)}+(\mu-\lambda) \operatorname{deg} E_{3}^{(4)} \\
& =-2(\lambda-\mu) \operatorname{deg} E_{1}^{(2)}-2 \mu \operatorname{deg} E_{2}^{(3)} \geq 0 .
\end{aligned}
$$

- $P_{6}$ : For this parabolic we have the filtration $\mathcal{E}=\left(0 \subset E_{1}^{(1)} \subset E_{2}^{(2)} \subset E_{3}^{(4)} \subset E_{4}^{(5)} \subset\right.$ $\mathbb{E})$, the set of weights $\Lambda(\mathcal{E})=\{(-\lambda,-\mu, 0, \mu, \lambda)\}$, the condition

$$
\phi=\left(\begin{array}{cccccc}
f_{1,1} & f_{1,2} & f_{1,3} & f_{1,4} & f_{1,5} & 0 \\
0 & f_{2,2} & f_{2,3} & f_{2,4} & 0 & -f_{1,5} \\
0 & 0 & f_{3,3} & 0 & -f_{2,4} & -f_{1,4} \\
0 & 0 & 0 & -f_{3,3} & -f_{2,3} & -f_{1,3} \\
0 & 0 & 0 & 0 & -f_{2,2} & -f_{1,2} \\
0 & 0 & 0 & 0 & 0 & -f_{1,1}
\end{array}\right),
$$

and the semistability condition implies

$$
\begin{aligned}
d(\mathcal{E}, \lambda) & =(-\lambda+\mu) \operatorname{deg} E_{1}^{(1)}-\mu \operatorname{deg} E_{2}^{(2)}-\mu \operatorname{deg} E_{3}^{(4)}+(\mu-\lambda) \operatorname{deg} E_{4}^{(5)} \\
& =-2(\lambda-\mu) \operatorname{deg} E_{1}^{(1)}-2 \mu \operatorname{deg} E_{2}^{(2)} \geq 0
\end{aligned}
$$


- $P_{7}$ : For this parabolic we have the filtration $\mathcal{E}=\left(0 \subset E_{1}^{(1)} \subset E_{2}^{(2)} \subset E_{3}^{(3)} \subset E_{4}^{(4)} \subset\right.$ $\left.E_{5}^{(5)} \subset \mathbb{E}\right)$, the set of weights $\Lambda(\mathcal{E})=\{(-\lambda,-\mu,-\gamma, \gamma, \mu, \lambda)\}$, the condition

$$
\phi=\left(\begin{array}{cccccc}
f_{1,1} & f_{1,2} & f_{1,3} & f_{1,4} & f_{1,5} & 0 \\
0 & f_{2,2} & f_{2,3} & f_{2,4} & 0 & -f_{1,5} \\
0 & 0 & f_{3,3} & 0 & -f_{2,4} & -f_{1,4} \\
0 & 0 & 0 & -f_{3,3} & -f_{2,3} & -f_{1,3} \\
0 & 0 & 0 & 0 & -f_{2,2} & -f_{1,2} \\
0 & 0 & 0 & 0 & 0 & -f_{1,1}
\end{array}\right),
$$

and the semistability condition implies

$$
\begin{aligned}
d(\mathcal{E}, \lambda)= & (-\lambda+\mu) \operatorname{deg} E_{1}^{(1)}+(-\mu+\gamma) \operatorname{deg} E_{2}^{(2)}+(-\gamma-\gamma) \operatorname{deg} E_{3}^{(3)}+ \\
& +(\gamma-\mu) \operatorname{deg} E_{4}^{(4)}+(\mu-\lambda) \operatorname{deg} E_{5}^{(5)}= \\
= & -2(\lambda-\mu) \operatorname{deg} E_{1}^{(1)}-2(\mu-\gamma) \operatorname{deg} E_{2}^{(2)}-2 \gamma \operatorname{deg} E_{3}^{(3)} \geq 0 .
\end{aligned}
$$

Then, $(\mathbb{E}, Q, \phi)$ is semistable if and only if the semistability condition holds for the parabolic subgroups $P_{1}, P_{2}$ and $P_{3}$, and in these cases, the condition $\phi \in H^{0}(N(\mathcal{E}, \lambda) \otimes K)$ is equivalent to $\phi\left(E^{\prime}\right) \subseteq E^{\prime} \otimes K$, where $E^{\prime}$ is the isotropic subbundle which has to verify $\operatorname{deg} E^{\prime} \leq 0$. The analogous property holds for the stability condition.

In fact, we have an inclusion $\mathfrak{p}_{3} \subset \mathfrak{p}_{1}$, and then, if we suppose that the condition holds for $P_{1}$, then it automatically holds for $P_{3}$. Therefore, it suffices to verify the stability condition for $P_{1}$ and $P_{2}$, which are the maximal parabolic subgroups of $\mathrm{SO}(6, \mathbb{C})$. This is a general phenomenon for $\mathrm{SO}(n, \mathbb{C})$ when $n$ is even. 



\section{$3 \quad \mathrm{SO}_{0}(p, q)$-Higgs bundles}

The special orthogonal group $\mathrm{SO}(p, q)$ is the subgroup of $\mathrm{SL}(n=p+q, \mathbb{R})$ consisting of all linear transformations of a $n=p+q$ dimensional real vector space which leave invariant a non-degenerate symmetric bilinear form of signature $(p, q)$. This group is a real form of $\mathrm{SO}(n=p+q, \mathbb{C})$.

Using the standard non-degenerate symmetric bilinear form of signature $(p, q)$ on $\mathbb{R}^{n}$

$$
\epsilon(x, y)=-x_{1} y_{1}-\cdots-x_{p} y_{p}+x_{p+1} y_{p+1}+\cdots+x_{n} y_{n}
$$

this means that,

$$
\epsilon(A x, A y)=\epsilon(x, y)
$$

for all $A \in \mathrm{SO}(p, q)$ and all $x, y \in \mathbb{R}^{n}$. Then

$$
\mathrm{SO}(p, q)=\left\{A \in \mathrm{SL}(n=p+q, \mathbb{R}) \mid A^{t} I_{p, q} A=I_{p, q}\right\},
$$

where $I_{p, q}=\left(\begin{array}{cc}-I_{p} & \\ & I_{q}\end{array}\right)$.

The Lie group $\mathrm{SO}(p, q)$ is a real (non-complex) group of dimension $n(n-1) / 2$ which is non-compact for $p, q \neq 0$, semisimple for $p+q \geq 3$ and which has two connected components. Let $\mathrm{SO}_{0}(p, q)$ be the connected component of the identity.

The Lie algebra of $\mathrm{SO}(p, q)$ and then of its identity component $\mathrm{SO}_{0}(p, q)$ is $\mathfrak{s o}(p, q)$, which has Cartan decomposition

$$
\mathfrak{s o}(p, q)=\mathfrak{h}+\mathfrak{m}
$$

where $\mathfrak{h}=\mathfrak{s o}(p) \times \mathfrak{s o}(q)$ is the Lie algebra of the maximal compact subgroup of $\mathrm{SO}_{0}(p, q)$. If we use the standard non-degenerate symmetric bilinear form of signature $(p, q)$, we have

$$
\begin{gathered}
\mathfrak{s o}(p, q)=\left\{X \in \mathfrak{s l}(n=p+q, \mathbb{R}) \mid X^{t} I_{p, q}+I_{p, q} X=0\right\}= \\
=\left\{\left(\begin{array}{cc}
X_{1} & X_{2} \\
X_{2}^{t} & X_{3}
\end{array}\right) \mid X_{1}, X_{3} \text { real skew-symmetric of rank } p \text { and } q, X_{2} \text { real }(p \times q)\right\},
\end{gathered}
$$

and then

$$
\mathfrak{h}=\left\{\left(\begin{array}{cc}
X_{1} & 0 \\
0 & X_{3}
\end{array}\right) \mid X_{1} \in \mathfrak{s o}(p), X_{3} \in \mathfrak{s o}(q)\right\},
$$

and

$$
\mathfrak{m}=\left\{\left(\begin{array}{cc}
0 & X_{2} \\
X_{2}^{t} & 0
\end{array}\right) \mid X_{2} \text { real }(p \times q) \text {-matrix }\right\}
$$


The involution of $\mathfrak{s o}(p+q, \mathbb{C})$ that defines $\mathfrak{s o}(p, q)$ as a real form is $\sigma(X)=I_{p, q} \bar{X} I_{p, q}$, that is

$$
\begin{gathered}
\mathfrak{s o}(p, q)=\left\{X \in \mathfrak{s o}(p+q, \mathbb{C}) \mid I_{p, q} \bar{X} I_{p, q}=X\right\}= \\
=\left\{X \in \mathfrak{s l}(p+q, \mathbb{C}) \mid X+X^{t}=0, I_{p, q} \bar{X} I_{p, q}=X\right\}= \\
=\left\{\left(\begin{array}{cc}
X_{1} & i X_{2} \\
-i X_{2}^{t} & X_{3}
\end{array}\right) \mid X_{1}, X_{3} \text { real skew-symmetric of rank } p \text { and } q, X_{2} \text { real }(p \times q)\right\} .
\end{gathered}
$$

Observe that there is an isomorphism

$$
\left(\begin{array}{cc}
X_{1} & i X_{2} \\
-i X_{2}^{t} & X_{3}
\end{array}\right) \rightarrow\left(\begin{array}{cc}
X_{1} & X_{2} \\
X_{2}^{t} & X_{3}
\end{array}\right)=\left(\begin{array}{cc}
-i I_{p} & 0 \\
0 & I_{q}
\end{array}\right)\left(\begin{array}{cc}
X_{1} & i X_{2} \\
-i X_{2}^{t} & X_{3}
\end{array}\right)\left(\begin{array}{cc}
i I_{p} & 0 \\
0 & I_{q}
\end{array}\right)
$$

The Cartan decomposition of the complex Lie algebra is

$$
\mathfrak{s o}(p+q, \mathbb{C})=(\mathfrak{s o}(p, \mathbb{C}) \times \mathfrak{s o}(q, \mathbb{C})) \oplus \mathfrak{m}^{\mathbb{C}}
$$

where

$$
\mathfrak{m}^{\mathbb{C}}=\left\{\left(\begin{array}{cc}
0 & X_{2} \\
-X_{2}^{t} & 0
\end{array}\right) \mid X_{2} \text { complex matrix }(p \times q)\right\},
$$

and the complexified isotropy representation is

$$
\iota: \mathrm{SO}(p, \mathbb{C}) \times \mathrm{SO}(q, \mathbb{C}) \rightarrow \mathrm{GL}\left(\mathfrak{m}^{\mathbb{C}}\right)
$$

where

$$
\begin{aligned}
\iota\left(\begin{array}{ll}
a & 0 \\
0 & b
\end{array}\right)\left(\begin{array}{cc}
0 & X_{2} \\
-X_{2}^{t} & 0
\end{array}\right) & =\left(\begin{array}{ll}
a & 0 \\
0 & b
\end{array}\right)\left(\begin{array}{cc}
0 & X_{2} \\
-X_{2}^{t} & 0
\end{array}\right)\left(\begin{array}{cc}
a^{-1} & 0 \\
0 & b^{-1}
\end{array}\right)= \\
& =\left(\begin{array}{cc}
0 & a X_{2} b^{-1} \\
-b X_{2}^{t} a^{-1} & 0
\end{array}\right) \in \mathfrak{m}^{\mathbb{C}}
\end{aligned}
$$

Definition 3.1. An $\mathrm{SO}_{0}(p, q)$-Higgs bundle is a pair $(E, \varphi)$, where $E$ is a holomorphic principal $\mathrm{SO}(p, \mathbb{C}) \times \mathrm{SO}(q, \mathbb{C})$-bundle over $X$ and $\varphi \in H^{0}\left(E\left(\mathfrak{m}^{\mathbb{C}}\right) \otimes K\right)$.

Two $\mathrm{SO}_{0}(p, q)$-Higgs bundles $(E, \varphi)$ and $\left(E^{\prime}, \varphi^{\prime}\right)$ are isomorphic if there is an isomorphism $E \cong E^{\prime}$ which takes $\varphi$ to $\varphi^{\prime}$ under the induced isomorphism $E\left(\mathfrak{m}^{\mathbb{C}}\right) \cong E^{\prime}\left(\mathfrak{m}^{\mathbb{C}}\right)$.

If $(E, \varphi)$ is an $\mathrm{SO}_{0}(p, q)$-Higgs bundle, the principal $\mathrm{SO}(p, \mathbb{C}) \times \mathrm{SO}(q, \mathbb{C})$-bundle $E$ is the fibred product

$$
E=E_{\mathrm{SO}(p, \mathbb{C})} \times E_{\mathrm{SO}(q, \mathbb{C})}
$$

of two principal bundles with structure groups $\mathrm{SO}(p, \mathbb{C})$ and $\mathrm{SO}(q, \mathbb{C})$ respectively. Using the standard representations of $\mathrm{SO}(p, \mathbb{C})$ and $\mathrm{SO}(q, \mathbb{C})$ in $\mathbb{C}^{p}$ and $\mathbb{C}^{q}$ we can associate to 
$E_{\mathrm{SO}(p, \mathbb{C})}$ and $E_{\mathrm{SO}(q, \mathbb{C})}$ two holomorphic vector bundles $V$ and $W$ of rank $p$ and $q$ respectively,

$$
\begin{aligned}
& V=E_{\mathrm{SO}(p, \mathbb{C})} \times_{\mathrm{SO}(p, \mathbb{C})} \mathbb{C}^{p}, \\
& W=E_{\mathrm{SO}(q, \mathbb{C})} \times_{\mathrm{SO}(q, \mathbb{C})} \mathbb{C}^{q},
\end{aligned}
$$

with $\operatorname{det} V=\operatorname{det} W=\mathcal{O}$, together with two non-degenerate symmetric quadratic forms

$$
Q_{V}: V \otimes V \rightarrow \mathbb{C} \text { and } Q_{W}: W \otimes W \rightarrow \mathbb{C}
$$

which induce two isomorphisms

$$
q_{V}: V \stackrel{\sim}{\longrightarrow} V^{*} \text { and } q_{W}: W \stackrel{\sim}{\longrightarrow} W^{*} .
$$

The vector bundle $E\left(\mathfrak{m}^{\mathbb{C}}\right)$ can be expressed in terms of $V$ and $W$ as follows:

$$
E\left(\mathfrak{m}^{\mathbb{C}}\right)=\left\{(\eta, \nu) \in \operatorname{Hom}(W, V) \oplus \operatorname{Hom}(V, W) \mid \nu=-\eta^{\top}\right\}
$$

where $\eta^{\top}=q_{W}^{-1} \circ \eta^{t} \circ q_{V}$,

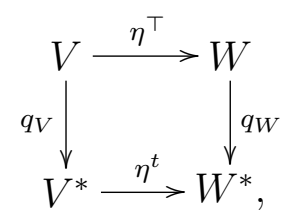

that is, $E\left(\mathfrak{m}^{\mathbb{C}}\right) \cong \operatorname{Hom}(W, V)$. Then, in terms of vector bundles, the Higgs field is a section $\eta \in H^{0}(\operatorname{Hom}(W, V) \otimes K)$, that is

$$
\eta: W \rightarrow V \otimes K
$$

and hence $\mathrm{SO}_{0}(p, q)$-Higgs bundles $(E, \varphi)$ are in one-to-one correspondence with tuples $\left(V, Q_{V}, W, Q_{W}, \eta\right)$.

Finally, an isomorphism between two $\mathrm{SO}_{0}(p, q)$-Higgs bundles $\left(V, Q_{V}, W, Q_{W}, \eta\right)$ and $\left(V^{\prime}, Q_{V^{\prime}}, W^{\prime}, Q_{W^{\prime}}, \eta^{\prime}\right)$ is given by two isomorphisms $g_{V}: V \rightarrow V^{\prime}$ and $g_{W}: W \rightarrow W^{\prime}$ such that $\left(g_{V} \times I_{K}\right) \circ \eta=\eta^{\prime} \circ g_{W}$, where $I_{K}$ is the identity on $K$.

\subsection{Stability of $\mathrm{SO}_{0}(p, q)$-Higgs bundles}

Every parabolic subgroup $P$ of $\mathrm{SO}(p, \mathbb{C}) \times \mathrm{SO}(q, \mathbb{C})$ is of the form $P_{1} \times P_{2}$, where $P_{1} \subset$ $\mathrm{SO}(p, \mathbb{C})$ and $P_{2} \subset \mathrm{SO}(q, \mathbb{C})$ are parabolic subgroups.

Given a $s=\left(s_{1}, s_{2}\right) \in i(\mathfrak{s o}(p) \times \mathfrak{s o}(q))$, we define the sets

$$
P_{s_{1}}=\left\{g \in \mathrm{SO}(p, \mathbb{C}) \mid e^{t s_{1}} g e^{-t s_{1}} \text { is bounded as } t \rightarrow \infty\right\}
$$




$$
P_{s_{2}}=\left\{g \in \mathrm{SO}(q, \mathbb{C}) \mid e^{t s_{2}} g e^{-t s_{2}} \text { is bounded as } t \rightarrow \infty\right\}
$$

These sets are parabolic subgroups of $\mathrm{SO}(p, \mathbb{C})$ and $\mathrm{SO}(q, \mathbb{C})$ respectively and then $P_{s_{1}} \times$ $P_{s_{2}}$ is a parabolic subgroup of the product. In fact, every parabolic subgroup of $\operatorname{SO}(p, \mathbb{C}) \times$ $\mathrm{SO}(q, \mathbb{C})$ is of this form.

Let $(E, \varphi)$ be an $\mathrm{SO}_{0}(p, q)$-Higgs bundle and consider a reduction of structure group for the principal $\mathrm{SO}(p, \mathbb{C}) \times \mathrm{SO}(q, \mathbb{C})$-bundle $E$ to a parabolic subgroup $P_{1} \times P_{2}$ of $\mathrm{SO}(p, \mathbb{C}) \times$ $\mathrm{SO}(q, \mathbb{C})$. Let $V$ and $W$ be the pair of holomorphic vector bundles associated to $E$ and let $\left(s_{1}, s_{2}\right)$ be the pair of elements in $i \mathfrak{s o}(p) \times i \mathfrak{s o}(q)$ associated to $P_{1}$ and $P_{2}$. If $-\lambda_{1}<\ldots<-\lambda_{r}<0<\lambda_{r}<\ldots<\lambda_{1}$ and $-\mu_{1}<\ldots<-\mu_{s}<0<\mu_{s}<\ldots<\mu_{1}$ are the sequences of different eigenvalues for $s_{1}$ and $s_{2}$ (we use again $r$ and $s$ ), where the zeros appear only if $p$ or $q$ are odd, let $V\left(\lambda_{j}\right)=\operatorname{ker}\left(\lambda_{j}-s_{1}\right), W\left(\lambda_{j}\right)=\operatorname{ker}\left(\mu_{j}-s_{2}\right)$ and $V_{i}=\bigoplus_{j \leq i} V\left(\lambda_{j}\right), W_{i}=\bigoplus_{j \leq i} W\left(\mu_{j}\right)$. With these definitions we obtain filtrations

$$
\begin{gathered}
\mathcal{V}=\left(0 \subset V_{1} \subset \cdots \subset V_{r}=V\right), \\
\mathcal{W}=\left(0 \subset W_{1} \subset \cdots \subset W_{s}=W\right),
\end{gathered}
$$

of isotropic subbundles generated by the eigenvectors in which $s=\left(s_{1}, s_{2}\right)$ is diagonal. The parabolic subgroups $P_{s_{1}}$ and $P_{s_{2}}$ are precisely the subgroups which preserve these filtrations.

Applying Definition 1.2 to $\mathrm{SO}_{0}(p, q)$-Higgs bundles and using the language of filtrations described in Section 1.3 we obtain the following result.

Proposition 3.2. Let $\left(V, W, Q_{V}, Q_{W}, \eta\right)$ be an $\mathrm{SO}_{0}(p, q)$-Higgs bundle with $p, q \neq 2$, then it is semistable if for any pair of filtrations

$$
\begin{gathered}
\mathcal{V}=\left(0 \subset V_{1} \subset \cdots \subset V_{r}=V\right), \\
\mathcal{W}=\left(0 \subset W_{1} \subset \cdots \subset W_{s}=W\right),
\end{gathered}
$$

satisfying $V_{j}=V_{r-j}^{\perp_{Q_{V}}}, W_{j}=V_{s-j}^{\perp_{Q_{W}}}$ and any element $(\lambda, \mu) \in \Lambda(\mathcal{V}) \times \Lambda(\mathcal{W})$ with

$$
\begin{aligned}
& \Lambda(\mathcal{V})=\left\{\lambda=\left(\lambda_{1}, \lambda_{2}, \ldots, \lambda_{r}\right) \in \mathbb{R}^{r} \mid \lambda_{i} \leq \lambda_{i+1}, \lambda_{r-i+1}+\lambda_{i}=0 \text { for any } i\right\}, \\
& \Lambda(\mathcal{W})=\left\{\mu=\left(\mu_{1}, \mu_{2}, \ldots, \mu_{s}\right) \in \mathbb{R}^{s} \mid \mu_{i} \leq \mu_{i+1}, \mu_{s-i+1}+\mu_{i}=0 \text { for any } i\right\},
\end{aligned}
$$

such that $\eta \in H^{0}(N \otimes K)$, where

$$
N=N(\mathcal{V}, \mathcal{W}, \lambda, \mu)=\sum_{\lambda_{j} \leq \mu_{i}} \operatorname{Hom}\left(W_{i}, V_{j}\right)
$$


we have

$$
d(\mathcal{V}, \lambda)+d(\mathcal{W}, \mu) \geq 0
$$

The tuple $\left(V, W, Q_{V}, Q_{W}, \eta\right)$ is stable if it is semistable and for any choice of the filtrations $(\mathcal{V}, \mathcal{W})$ and non-zero $(\lambda, \mu) \in \Lambda(\mathcal{V}) \times \Lambda(\mathcal{W})$, that is, at least one of $\lambda$ and $\mu$ is non-zero, such that $\eta \in H^{0}(N \otimes K)$, we have

$$
d(\mathcal{V}, \lambda)+d(\mathcal{W}, \mu)>0
$$

Finally, the tuple $\left(V, W, Q_{V}, Q_{W}, \eta\right)$ is polystable if it is semistable and for any filtrations $(\mathcal{V}, \mathcal{W})$ as above and non-zero $(\lambda, \mu) \in \Lambda(\mathcal{V}) \times \Lambda(\mathcal{W})$ satisfying $\lambda_{i}<\lambda_{i+1}, \mu_{i}<\mu_{i+1}$ for each $i, \eta \in H^{0}(N \otimes K)$ and $d(\mathcal{V}, \lambda)+d(\mathcal{W}, \mu)=0$, there are splittings of vector bundles

$$
V \simeq V_{1} \oplus V_{2} / V_{1} \oplus \cdots \oplus V / V_{r-1} \quad W \simeq W_{1} \oplus W_{2} / W_{1} \oplus \cdots \oplus W / W_{s-1}
$$

satisfying

$$
\begin{gathered}
Q_{V}\left(V_{i} / V_{i-1}, V_{j} / V_{j-1}\right)=0 \text { unless } i+j=r+1, \\
Q_{W}\left(W_{i} / W_{i-1}, W_{j} / W_{j-1}\right)=0 \text { unless } i+j=s+1,
\end{gathered}
$$

with respect to which

$$
\eta \in H^{0}\left(\bigoplus_{\mu_{i}=\lambda_{j}} \operatorname{Hom}\left(W_{i} / W_{i-1}, V_{j} / V_{j-1}\right) \otimes K\right)
$$

The following proposition contains the simplified notions of semistability and stability.

Proposition 3.3. Let $\left(V, Q_{V}, W, Q_{W}, \eta\right)$ be an $\mathrm{SO}_{0}(p, q)$-Higgs bundle with $p, q \neq 2$. It is semistable if and only if for any pair of isotropic subbundles $V^{\prime} \subset V, W^{\prime} \subset W$ such that $\eta\left(W^{\prime}\right) \subseteq V^{\prime} \otimes K$, the inequality $\operatorname{deg} V^{\prime}+\operatorname{deg} W^{\prime} \leq 0$ holds. It is stable if and only if it is semistable and for any pair of isotropic subbundles $V^{\prime} \subset V, W^{\prime} \subset W$, at least one of them non-zero, such that $\eta\left(W^{\prime}\right) \subseteq V^{\prime} \otimes K$, we have $\operatorname{deg} V^{\prime}+\operatorname{deg} W^{\prime}<0$.

Proof. Take an $\mathrm{SO}_{0}(p, q)$-Higgs bundle $\left(V, Q_{V}, W, Q_{W}, \eta\right)$ and assume that for any pair of isotropic subbundles $V^{\prime} \subseteq V, W^{\prime} \subset W$ such that $\eta\left(W^{\prime}\right) \subset V^{\prime} \otimes K$, we have $\operatorname{deg} V^{\prime}+$ $\operatorname{deg} W^{\prime} \leq 0$ holds. We want to prove that $\left(V, Q_{V}, W, Q_{W}, \eta\right)$ is semistable.

Choose a pair of filtrations $\mathcal{V}=\left(0 \subset V_{1} \subset \ldots \subset V_{r}=V\right.$ and $\mathcal{W}=\left(0 \subset W_{1} \subset \ldots \subset\right.$ $W_{s}=W$ satisfying $V_{j}=V_{r-j}^{\perp_{Q}}$ and $W_{j}=W_{s-j}^{\perp_{Q_{W}}}$ for any $j$. We have to understand the geometry of the convex set

$$
\Lambda=\{(\lambda, \mu) \in \Lambda(\mathcal{V}) \times \Lambda(\mathcal{W}) \mid \eta \in N\} \subset \mathbb{R}^{r} \times \mathbb{R}^{s}
$$


Let

$$
\begin{aligned}
\mathcal{J} & =\left\{(i, j) \mid \eta\left(W_{j}\right) \subseteq V_{i} \otimes K,\left(\eta\left(W_{j}\right) \nsubseteq V_{k} \otimes K \text { for } l<i\right)\right\} \\
& =\left\{\left(i_{1}, j_{1}\right), \ldots,\left(i_{k}, j_{k}\right)\right\}
\end{aligned}
$$

One checks easily that if $(\lambda, \mu) \in \Lambda(\mathcal{V}) \times \Lambda(\mathcal{W})$, then

$$
(\lambda, \mu) \in \Lambda \Leftrightarrow \begin{cases}\lambda_{a}=\lambda_{b}, & \text { for any } i_{l} \leq a \leq b \leq i_{l+1}, \\ \mu_{a}=\mu_{b}, & \text { for any } j_{l} \leq a \leq b \leq j_{l+1} .\end{cases}
$$

The set of indices $\mathcal{J}$ is symmetric,

$$
(i, j) \in \mathcal{J} \Leftrightarrow(r-i, s-j) \in \mathcal{J}
$$

To check this we have to prove that $\phi\left(E_{j}\right) \subseteq E_{j} \otimes K$ implies that $\phi\left(E_{j}^{\perp_{Q}}\right) \subseteq E_{j}^{\perp_{Q}} \otimes$ $K$. Suppose that this is not true, then there is a pair $(i, j)$ with $\eta\left(W_{j}\right) \subseteq V_{i} \otimes K$ (or equivalently, $\left.\eta^{\top}\left(V_{i}\right) \subseteq W_{j} \otimes K\right)$ and there exists some $w \in W_{j}^{\perp_{Q_{W}}}$ such that $\eta(w) \notin$ $V_{i}^{\perp_{Q_{V}}} \otimes K$. Then there exists $v \in V_{i}$ such that $Q_{V}(v, \eta(w)) \neq 0$. We have

$$
Q_{V}(v, \eta(w))=Q_{W}\left(-\eta^{\top}(v), w\right)=0
$$

and the latter vanishes because by assumption $-\eta^{\top}(v)$ belongs to $W_{j}$. So we have reached a contradiction.

Let $\mathcal{J}^{\prime}=\{(i, j) \in \mathcal{J} \mid 2 i \leq r, 2 j \leq s\}$ and define for any $(i, j) \in \mathcal{J}^{\prime}$ the vectors

$$
\begin{aligned}
L_{i} & =-\sum_{c \leq i} e_{c}+\sum_{d \geq r-i+1} e_{d}, \\
M_{j} & =-\sum_{c \leq j} f_{c}+\sum_{d \geq s-j+1} f_{d},
\end{aligned}
$$

where $\left\{e_{1}, \ldots, e_{r}\right\}$ and $\left\{f_{1}, \ldots, f_{s}\right\}$ are the canonical basis of $\mathbb{R}^{r}$ and $\mathbb{R}^{s}$. The set $\Lambda$ is the positive span of the vectors $\left\{L_{i}, M_{j} \mid(i, j) \in \mathcal{J}^{\prime}\right\}$ and we have

$$
d(\mathcal{V}, \lambda)+d(\mathcal{W}, \mu) \geq 0 \text { for any }(\lambda, \mu) \in \Lambda \Leftrightarrow d\left(\mathcal{V}, L_{i}\right)+d\left(\mathcal{W}, M_{j}\right) \geq 0 \text { for any }(i, j) .
$$

We have also that

$$
\begin{gathered}
d\left(\mathcal{V}, L_{i}\right)=-\operatorname{deg} V_{r-i}-\operatorname{deg} V_{i}, \\
d\left(\mathcal{W}, M_{j}\right)=-\operatorname{deg} W_{s-j}-\operatorname{deg} W_{j} .
\end{gathered}
$$

Since $\operatorname{deg} V_{r-i}=\operatorname{deg} V_{i}$ and $\operatorname{deg} W_{s-j}=\operatorname{deg} W_{j}$, then $d\left(\mathcal{V}, L_{i}\right)+d\left(\mathcal{W}, M_{j}\right)=-2\left(\operatorname{deg} V_{i}+\right.$ $\left.\operatorname{deg} W_{j}\right) \geq 0$ is equivalent to $\operatorname{deg} V_{i}+\operatorname{deg} W_{j} \leq 0$, which holds by assumption. Hence $\left(V, Q_{V}, W, Q_{W}, \eta\right)$ is semistable. 
Conversely, if $\left(V, Q_{V}, W, Q_{W}, \eta\right)$ is semistable, for any isotropic subbundle $E^{\prime} \subset \mathbb{E}$ such that $\phi\left(E^{\prime}\right) \subseteq E^{\prime} \otimes K$ we have that the condition $\operatorname{deg} E^{\prime} \leq 0$ is immediate by applying the semistability condition of the filtrations $0 \subset V^{\prime} \subset V^{\prime \perp_{Q_{V}}} \subset V$ and $0 \subset W^{\prime} \subset W^{\prime \perp_{Q_{W}}} \subset W$.

Finally, the proof of the second statement on stability is very similar to case of semistability, so we omit it.

As we have mentioned in Section 2.2, the Lie group $\mathrm{SO}(2, \mathbb{C})$ has no parabolic subgroups and the stability condition can not be simplified in terms of isotropic subbundles. This phenomenon appears also for $\mathrm{SO}_{0}(p, q)$-Higgs bundles in the study of the parabolic subgroups of $\mathrm{SO}(p, \mathbb{C}) \times \mathrm{SO}(q, \mathbb{C})$ when $p$ or $q$ are equal to 2 . This is the reason why Proposition 3.3 does not work when $p=2$ or $q=2$. The following result deals with this case.

Proposition 3.4. An $\mathrm{SO}_{0}(2, q)$-Higgs bundle $\left(V, Q_{V}, W, Q_{W}, \eta\right)$ is semistable if and only if for any isotropic subbundle $W^{\prime} \subset W$ such that $\eta\left(W^{\prime}\right) \subseteq V \otimes K$, the inequality $\operatorname{deg} W^{\prime} \leq 0$ holds. It is stable if and only if it is semistable and for non-zero isotropic subbundle $W^{\prime} \subset W$ such that $\eta\left(W^{\prime}\right) \subseteq V \otimes K$, we have $\operatorname{deg} W^{\prime}<0$.

To conclude this section we include several low rank examples to illustrate the notions of stability for $\mathrm{SO}_{0}(p, q)$-Higgs bundles. In Example 3.5 we deduce the simplified notion of stability given by Proposition 3.3 in the particular case of $\mathrm{SO}_{0}(3,3)$-Higgs bundles. In Example 3.6, using the low rank isomorphisms given in Section 5, we compare the notion of stability for the special orthogonal group $\mathrm{SO}_{0}(2,1)$ with the notion of stability for $\operatorname{Sp}(2, \mathbb{R})$ defined in [19].

Example 3.5. Stability of $\mathrm{SO}_{0}(3,3)$-Higgs bundles.

Let $(E, \varphi)$ be an $\mathrm{SO}_{0}(3,3)$-Higgs bundle. The parabolic subgroups of $\mathrm{SO}(3, \mathbb{C})$ are $T=$ $\mathrm{SO}(3, \mathbb{C})$ and $P_{\lambda}=\left(\begin{array}{rrr}* & * & * \\ & * & * \\ & & *\end{array}\right) \subset \mathrm{SO}(3, \mathbb{C})$ corresponding to the elements $s=0$ and $s=$ $\left(\begin{array}{ccc}-\lambda & & \\ & 0 & \\ & & \lambda\end{array}\right)$ in $i \mathfrak{s o}(3)$. A reduction of the structure group of the principal $\operatorname{SO}(3, \mathbb{C}) \times$ $\mathrm{SO}(3, \mathbb{C})$-bundle $E$ to a parabolic subgroup of $\mathrm{SO}(3, \mathbb{C}) \times \mathrm{SO}(3, \mathbb{C})$ is in correspondence with a pair of filtrations of the associated vector bundles $V$ and $W$. This correspondence 
is the following:

$$
\begin{aligned}
& T=T \times T, \\
& (0,0) \text {, } \\
& P_{1}=P_{\lambda} \times T, \\
& \mathcal{V}=(0 \subset V), \mathcal{W}=(0 \subset W) \text {, } \\
& (-\lambda<0<\lambda, 0) \text {, } \\
& \mathcal{V}=\left(0 \subset V_{1}^{(1)} \subset V_{2}^{(2)} \subset V\right), \mathcal{W}=(0 \subset W), \\
& P_{2}=T \times P_{\lambda}, \\
& (0,-\lambda<0<\lambda) \text {, } \\
& \mathcal{V}=(0 \subset V), \mathcal{W}=\left(0 \subset W_{1}^{(1)} \subset W_{2}^{(2)} \subset W\right), \\
& P_{3}=P_{\lambda} \times P_{\mu}, \\
& (-\lambda<0<\lambda,-\mu<0<\mu) \text {, } \\
& \mathcal{V}=\left(0 \subset V_{1}^{(1)} \subset V_{2}^{(2)} \subset V\right), \mathcal{W}=\left(0 \subset W_{1}^{(1)} \subset W_{2}^{(2)} \subset W\right)
\end{aligned}
$$

In this example, we use two different methods to write the condition for the parabolic subgroups given by the Higgs field, we use the definition

$$
N=\sum_{\lambda_{j} \leq \mu_{i}} \operatorname{Hom}\left(W_{i}, V_{j}\right)
$$

and we also compute the set

$$
\left\{X \in \mathfrak{m}^{\mathbb{C}} \mid \iota\left(e^{t s}\right)(X) \text { is bounded as } t \rightarrow \infty\right\} .
$$

If $f=\left(f_{i j}\right) \in \operatorname{Hom}(W, V) \cong \mathfrak{m}^{\mathbb{C}}$ and $\iota: \mathrm{SO}(3, \mathbb{C}) \times \mathrm{SO}(3, \mathbb{C}) \rightarrow \mathrm{GL}\left(\mathfrak{m}^{\mathbb{C}}\right)$ is the isotropy representation, then

$$
\begin{aligned}
\iota\left(e^{t s}\right)(f) & =\left(\begin{array}{cccc}
e^{-t \lambda} & & & \\
& 1 & & \\
& & e^{t \lambda}
\end{array}\right)\left(\begin{array}{ccc}
f_{1,1} & f_{1,2} & f_{1,3} \\
f_{2,1} & f_{2,2} & f_{2,3} \\
f_{3,1} & f_{3,2} & f_{3,3}
\end{array}\right)\left(\begin{array}{ccc}
e^{t \mu} & & \\
& 1 & \\
& e^{-t \mu}
\end{array}\right) \\
& =\left(\begin{array}{cccc}
e^{t(\mu-\lambda)} f_{1,1} & e^{-t \lambda} f_{1,2} & e^{-t(\mu+\lambda)} & f_{1,3} \\
& & & \\
e^{t \mu} f_{1,1} & f_{2,2} & & e^{-t \mu} f_{2,3} \\
e^{t(\mu+\lambda)} f_{3,1} & e^{t \lambda} f_{3,2} & & e^{t(\lambda-\mu)} f_{3,3}
\end{array}\right) .
\end{aligned}
$$

Observe that $s=\left(s_{1}, s_{2}\right)$ with $s_{1}=\left(\begin{array}{ccc}-\lambda & & \\ & 0 & \\ & & \lambda\end{array}\right)$ and $s_{2}=\left(\begin{array}{ccc}-\mu & & \\ & 0 & \\ & & \mu\end{array}\right)$ and that we are considering $\mathfrak{m}^{\mathbb{C}} \cong \operatorname{Hom}(W, V)$. If $f=\left(f_{i j}\right)$ is in $\operatorname{Hom}(W, V)$, the corresponding part in $\operatorname{Hom}(V, W)$ is

$$
f^{\top}=\left(\begin{array}{ccc}
f_{3,3} & f_{2,3} & f_{1,3} \\
f_{3,2} & f_{2,2} & f_{1,2} \\
f_{3,1} & f_{2,1} & f_{1,1}
\end{array}\right) .
$$

In the following list we describe, for each parabolic subgroup, the bundle $N$ and the condition for $d(\mathcal{V}, \lambda)+d(\mathcal{W}, \mu)$ that semistability condition requires: 
- $T: N=\operatorname{Hom}(W, V)$ and $d(\mathcal{V}, \lambda)=d(\mathcal{W}, \mu)=0$.

- $P_{1}: N=\operatorname{Hom}\left(W, V_{1}\right) \oplus \operatorname{Hom}\left(W, V_{2}\right)$ or equivalently $\eta=\left(\begin{array}{ccc}f_{1,1} & f_{1,2} & f_{1,3} \\ f_{2,1} & f_{2,2} & f_{2,3} \\ 0 & 0 & 0\end{array}\right)$, that is, $\eta^{\top}\left(V_{1}^{(1)}\right)=0$.

$d(\mathcal{V}, \lambda)=-2 \lambda \operatorname{deg} V_{1}^{(1)}, d(\mathcal{W}, \mu)=0$, then $d(\mathcal{V}, \lambda)+d(\mathcal{W}, \mu) \geq 0$ if and only if $\operatorname{deg} V_{1}^{(1)} \leq 0$.

- $P_{2}: N=\operatorname{Hom}\left(W_{2}, V\right) \oplus \operatorname{Hom}(W, V)$ or equivalently $\eta=\left(\begin{array}{ccc}0 & f_{1,2} & f_{1,3} \\ 0 & f_{2,2} & f_{2,3} \\ 0 & f_{3,2} & f_{3,3}\end{array}\right)$, that is, $\eta\left(W_{1}^{(1)}\right)=0$.

$d(\mathcal{V}, \lambda)=0, d(\mathcal{W}, \mu)=-2 \mu \operatorname{deg} W_{1}^{(1)}$, then $d(\mathcal{V}, \lambda)+d(\mathcal{W}, \mu) \geq 0$ if and only if $\operatorname{deg} W_{1}^{(1)} \leq 0$.

- $P_{3}, \lambda>\mu: N=\operatorname{Hom}\left(W_{1}, V_{1}\right) \oplus \operatorname{Hom}\left(W_{2}, V_{1}\right) \oplus \operatorname{Hom}\left(W_{2}, V_{2}\right) \oplus \operatorname{Hom}\left(W, V_{1}\right) \oplus$ $\operatorname{Hom}\left(W, V_{2}\right)$ or equivalently $\eta=\left(\begin{array}{ccc}f_{1,1} & f_{1,2} & f_{1,3} \\ 0 & f_{2,2} & f_{2,3} \\ 0 & 0 & 0\end{array}\right)$, that is, $\eta\left(W_{1}^{(1)}\right) \subseteq V_{1}^{(1)} \otimes K$ and $\eta^{\top}\left(V_{1}^{(1)}\right)=0$

$d(\mathcal{V}, \lambda)+d(\mathcal{W}, \mu)=-2 \lambda \operatorname{deg} V_{1}^{(1)}-2 \mu \operatorname{deg} W_{1}^{(1)}>-2 \lambda\left(\operatorname{deg} V_{1}^{(1)}+\operatorname{deg} W_{1}^{(1)}\right)$, then $d(\mathcal{V}, \lambda)+d(\mathcal{W}, \mu) \geq 0$ if and only if $\operatorname{deg} V_{1}^{(1)}+\operatorname{deg} W_{1}^{(1)} \leq 0$.

- $P_{3}, \lambda<\mu: \quad N=\operatorname{Hom}\left(W_{2}, V_{1}\right) \oplus \operatorname{Hom}\left(W_{2}, V_{2}\right) \oplus \operatorname{Hom}\left(W, V_{1}\right) \oplus \operatorname{Hom}\left(W, V_{2}\right) \oplus$ $\operatorname{Hom}(W, V)$ or equivalently $\eta=\left(\begin{array}{ccc}0 & f_{1,2} & f_{1,3} \\ 0 & f_{2,2} & f_{2,3} \\ 0 & 0 & f_{3,3}\end{array}\right)$, that is, $\eta\left(W_{1}^{(1)}\right)=0$ and $\eta^{\top}\left(V_{1}^{(1)}\right) \subseteq$ $W_{1}^{(1)} \otimes K$

$d(\mathcal{V}, \lambda)+d(\mathcal{W}, \mu)=-2 \lambda \operatorname{deg} V_{1}^{(1)}-2 \mu \operatorname{deg} W_{1}^{(1)}>-2 \mu\left(\operatorname{deg} V_{1}^{(1)}+\operatorname{deg} W_{1}^{(1)}\right)$, then $d(\mathcal{V}, \lambda)+d(\mathcal{W}, \mu) \geq 0$ if and only if $\operatorname{deg} V_{1}^{(1)}+\operatorname{deg} W_{1}^{(1)} \leq 0$.

- $P_{3}, \lambda=\mu: \quad N=\operatorname{Hom}\left(W_{1}, V_{1}\right) \oplus \operatorname{Hom}\left(W_{2}, V_{1}\right) \oplus \operatorname{Hom}\left(W_{2}, V_{2}\right) \oplus \operatorname{Hom}\left(W, V_{1}\right) \oplus$ $\operatorname{Hom}\left(W, V_{2}\right) \oplus \operatorname{Hom}(W, V)$ or equivalently $\eta=\left(\begin{array}{ccc}f_{1,1} & f_{1,2} & f_{1,3} \\ 0 & f_{2,2} & f_{2,3} \\ 0 & 0 & f_{3,3}\end{array}\right)$, that is, $\eta\left(W_{1}^{(1)}\right) \subseteq$ $V_{1}^{(1)} \otimes K$ and $\eta^{\top}\left(V_{1}^{(1)}\right) \subseteq W_{1}^{(1)} \otimes K$

$$
d(\mathcal{V}, \lambda)+d(\mathcal{W}, \lambda)=-2 \lambda\left(\operatorname{deg} V_{1}^{(1)}+\operatorname{deg} W_{1}^{(1)}\right), \text { then } d(\mathcal{V}, \lambda)+d(\mathcal{W}, \mu) \geq 0 \text { if and only }
$$
if $\operatorname{deg} V_{1}^{(1)}+\operatorname{deg} W_{1}^{(1)} \leq 0$. 
Observe that the $\mathrm{SO}_{0}(3,3)$-Higgs bundle is semistable if and only if the semistability condition holds for the parabolic subgroups $P_{1}$ and $P_{2}$, which are the maximal parabolic subgroups. The same happens for the stability condition (but we do not consider the parabolic $P$ ). For the parabolic subgroups $P_{1}$ and $P_{2}$ the condition given by the Higgs field is $\eta\left(W^{\prime}\right)=0$, where $W^{\prime}$ is the isotropic subbundle of $W$ which verify $\operatorname{deg} W^{\prime} \leq 0$ and respectively $\eta^{\top}\left(V^{\prime}\right)=0$, where $V^{\prime}$ is the isotropic subbundle of $V$ which has to verify $\operatorname{deg} V^{\prime} \leq 0$. For a pair of isotropic subbundles $V^{\prime}$ and $W^{\prime}$ in $V$ and $W$ respectively, the condition given by the Higgs field is $\eta\left(W^{\prime}\right) \subseteq V^{\prime} \otimes K$ (and $\left.\eta^{\top}\left(V^{\prime}\right) \subseteq W^{\prime} \otimes K\right)$ and the condition for the degrees is $\operatorname{deg} V^{\prime}+\operatorname{deg} W^{\prime} \leq 0$. Then, we have the result that we expect (Proposition 3.3).

Example 3.6. Stability of $\mathrm{SO}_{0}(2,1)$ and $\mathrm{Sp}(2, \mathbb{R})$-Higgs bundles.

In Section 5.1 we describe an isomorphism between the Lie group $\operatorname{Spin}(2,1)$, which is a double cover of $\mathrm{SO}_{0}(2,1)$, and the groups $\operatorname{SL}(2, \mathbb{R}) \cong \operatorname{Sp}(2, \mathbb{R})$, that allow us to define an arrow from the moduli space of $\mathrm{SL}(2, \mathbb{R})$-Higgs bundles to $\mathcal{M}\left(\mathrm{SO}_{0}(2,1)\right)$. In this example we check that, if an $\mathrm{SO}_{0}(2,1)$-Higgs bundle lifts to an $\mathrm{SL}(2, \mathbb{R})$-Higgs bundle, both stability notions coincide. This is due to the fact that in both cases the complexification of the maximal compact subgroups is $\mathrm{SO}(2, \mathbb{C})$ which has no proper parabolic subgroups.

\subsection{Polystable $\mathrm{SO}_{0}(p, q)$-Higgs bundles}

The main result in this section is Theorem 3.7 which give a full description of polystable $\mathrm{SO}_{0}(p, q)$-Higgs bundles.

For this, we need to describe some special $\mathrm{SO}_{0}(p, q)$-Higgs bundles which arise from certain $G$-Higgs bundles, where $G$ is a real subgroup of $\mathrm{SO}_{0}(p, q)$. We have the following inclusions:

$$
\begin{aligned}
\mathrm{U}(n) & \hookrightarrow \mathrm{SO}_{0}(2 n, 0) \text { or } \mathrm{SO}_{0}(0,2 n) \cong \mathrm{SO}(2 n) \\
V^{\prime} & \mapsto\left(V^{\prime} \oplus V^{\prime *},\langle\cdot, \cdot\rangle,-,-,-\right), \\
\mathrm{SO}(n) & \hookrightarrow \mathrm{SO}_{0}(n, 0) \text { or } \mathrm{SO}_{0}(0, n) \cong \mathrm{SO}(n) \\
\left(V^{\prime}, Q^{\prime}\right) & \mapsto\left(V^{\prime}, Q^{\prime},-,-,-\right), \\
\mathrm{U}(p, q) & \hookrightarrow \mathrm{SO}_{0}(2 p, 2 q) \\
\left(V^{\prime}, W^{\prime}, \beta^{\prime}, \gamma^{\prime}\right) & \mapsto\left(V^{\prime} \oplus V^{\prime *},\langle\cdot, \cdot\rangle, W^{\prime} \oplus W^{\prime *},\langle\cdot, \cdot\rangle, \beta^{\prime}+\gamma^{\prime t}\right),
\end{aligned}
$$


where $\langle\cdot, \cdot\rangle$ denotes the dual pairing in each case.

Theorem 3.7. Let $\left(V, Q_{V}, W, Q_{W}, \eta\right)$ be a polystable $\mathrm{SO}_{0}(p, q)$-Higgs bundle. There is a decomposition, unique up to reordering, of this Higgs bundle as a sum of stable $G_{i}$-Higgs bundles, where $G_{i}$ is one of the following groups: $\mathrm{SO}_{0}\left(p_{i}, q_{i}\right), \mathrm{U}\left(p_{i}, q_{i}\right), \mathrm{SO}\left(n_{i}\right)$ or $\mathrm{U}\left(n_{i}\right)$.

Proof. Let $\left(V, Q_{V}, W, Q_{W}, \eta\right)$ be a polystable $\mathrm{SO}_{0}(p, q)$-Higgs bundle. For the principal $\mathrm{SO}_{0}(p, \mathbb{C})$ and $\mathrm{SO}_{0}(q, \mathbb{C})$-bundles $\left(V, Q_{V}\right)$ and $\left(W, Q_{W}\right)$ we fix filtrations $\mathcal{V}=\left(0 \subset V_{1} \subset\right.$ $\left.\cdots \subset V_{r}=V\right), \mathcal{W}=\left(0 \subset W_{1} \subset \cdots \subset W_{s}=W\right)$, with $V_{j}=V_{r-j}^{\perp_{Q_{V}}}, W_{j}=W_{s-j}^{\perp_{Q_{W}}}$ and strictly antidominant characters $\lambda_{1}<\ldots<\lambda_{r}, \mu_{1}<\ldots<\mu_{s}$ with $\lambda_{r-i+1}+\lambda_{i}=0$, $\mu_{s-i+1}+\mu_{i}=0$, such that $\eta \in H^{0}\left(\bigoplus \operatorname{Hom}\left(W_{i}, V_{j}\right) \otimes K\right)$ and $d(\mathcal{V}, \lambda)+d(\mathcal{W}, \mu)=0$. Since $\left(V, Q_{V}, W, Q_{W}, \eta\right)$ is polystable, we have

$$
V \simeq V_{1} \oplus V_{2} / V_{1} \oplus \cdots \oplus V / V_{r-1}, \quad W \simeq W_{1} \oplus W_{2} / W_{1} \oplus \cdots \oplus W / W_{s-1}
$$

with

$$
\begin{gathered}
Q_{V}\left(V_{i} / V_{i-1}, V_{j} / V_{j-1}\right)=0 \text { unless } i+j=r+1, \\
Q_{W}\left(W_{i} / W_{i-1}, W_{j} / W_{j-1}\right)=0 \text { unless } i+j=s+1,
\end{gathered}
$$

and

$$
\eta \in H^{0}\left(\bigoplus_{\mu_{i}=\lambda_{j}} \operatorname{Hom}\left(W_{i} / W_{i-1}, V_{j} / V_{j-1}\right) \otimes K\right)
$$

The conditions

$$
\begin{gathered}
Q_{V}\left(V_{i} / V_{i-1}, V_{j} / V_{j-1}\right)=0 \text { unless } i+j=r+1, \\
Q_{W}\left(W_{i} / W_{i-1}, W_{j} / W_{j-1}\right)=0 \text { unless } i+j=s+1,
\end{gathered}
$$

tell us that the bilinear forms $Q_{V}$ and $Q_{W}$ give isomorphisms $\left(V_{i} / V_{i-1}\right)^{*} \cong V_{r-i+1} / V_{r-i}$ and $\left(W_{i} / W_{i-1}\right)^{*} \cong W_{s-i+1} / W_{s-i}$. We have the exact sequence

$$
V_{i}^{\perp} \longrightarrow V_{i-1}^{\perp} \stackrel{p}{\longrightarrow}\left(V_{i} / V_{i-1}\right)^{*}
$$

where $p$ is given by $v \mapsto Q_{V}(v, \cdot)$, then

$$
\left(V_{i} / V_{i-1}\right)^{*} \cong V_{i-1}^{\perp} / V_{i}^{\perp} \cong V_{r-i+1} / V_{r-i}
$$

The same for $\left(W, Q_{W}\right)$ and its corresponding decomposition.

Suppose that $p$ is odd and that we have a filtration $\mathcal{V}=\left(0 \subset V_{1} \subset \ldots \subset V_{r}=V\right)$, where $r$ is even, then $V_{\frac{r}{2}}^{\perp}=V_{r-\frac{r}{2}}=V_{\frac{r}{2}}$. On the other hand, $\operatorname{rk}\left(V_{\frac{r}{2}}^{\perp}\right)=p-\operatorname{rk}\left(V_{\frac{r}{2}}\right)$, that implies $\operatorname{rk}\left(V_{\frac{r}{2}}\right)=\frac{p}{2}$, which is not a natural number. Then, if $p$ is odd, all the possible 
filtrations $\mathcal{V}=\left(0 \subset V_{1} \subset \ldots \subset V_{r}=V\right)$, have odd length $r$, and the value 0 always appears in the middle of the corresponding strictly antidominant character $\lambda_{1}<\ldots<\lambda_{r}$. When the rank $p$ is even, we have filtrations for all $1 \leq r \leq p$. When $r$ is odd, we have $\lambda_{\frac{r+1}{2}}=0$ and in the even case, we have $\cdots \lambda_{\frac{r}{2}}<\lambda_{\frac{r}{2}+1} \cdots$, with $\lambda_{\frac{r}{2}}=-\lambda_{\frac{r}{2}+1}<0$. We have the analogous result when $q$ odd.

There is a piece of Higgs field non-equal to zero in the following cases:

- When $r$ and $s$ are odd and $\lambda_{\frac{r+1}{2}}=\mu_{\frac{s+1}{2}}=0$. Then we have

$$
\eta \in H^{0}\left(\operatorname{Hom}\left(W_{\frac{s+1}{2}} / W_{\frac{s-1}{2}}, V_{\frac{r+1}{2}} / V_{\frac{r-1}{2}}\right) \otimes K\right)
$$

and since

$$
\left(V_{\frac{r+1}{2}} / V_{\frac{r-1}{2}}\right)^{*} \cong V_{\frac{r+1}{2}} / V_{\frac{r-1}{2}} \text { and }\left(W_{\frac{s+1}{2}} / W_{\frac{s-1}{2}}\right)^{*} \cong W_{\frac{s+1}{2}} / W_{\frac{s-1}{2}},
$$

the piece

$$
\left(V_{\frac{r+1}{2}} / V_{\frac{r-1}{2}}, Q_{V}, W_{\frac{s+1}{2}} / W_{\frac{s-1}{2}}, Q_{W}, \eta\right)
$$

is in itself an $\mathrm{SO}_{0}\left(p^{\prime}, q^{\prime}\right)$-Higgs bundle with $p^{\prime} \leq p$ and $q^{\prime} \leq q$. Observe that $Q_{V}$ and $Q_{W}$ denotes now the restriction to the corresponding subbundles.

- When $\lambda_{j}=\mu_{i}$ for any $1 \leq j \leq r, 1 \leq i \leq s$. This implies also $\lambda_{r-j+1}=\mu_{s-i+1}$. Then we have

$$
\left(\eta_{1}, \eta_{2}\right) \in H^{0}\left(\left(\operatorname{Hom}\left(W_{i} / W_{i-1}, V_{j} / V_{j-1}\right) \oplus \operatorname{Hom}\left(W_{s-i+1} / W_{s-i}, V_{r-j+1} / V_{r-j}\right)\right) \otimes K\right)
$$

and since

$$
\left(V_{j} / V_{j-1}\right)^{*} \cong V_{r-j+1} / V_{r-j} \text { and }\left(W_{i} / W_{i-1}\right)^{*} \cong W_{s-i+1} / W_{s-i},
$$

the piece

$$
\left(V_{j} / V_{j-1}, W_{i} / W_{i-1}, \eta_{1}, \eta_{2}^{\top}\right)
$$

is in itself a $\mathrm{U}\left(p^{\prime}, q^{\prime}\right)$-Higgs bundle with $p^{\prime}<p$ and $q^{\prime}<q$. We can also take

$$
\left(V_{r-j+1} / V_{r-j}, W_{s-i+1} / W_{s-i}, \eta_{2}, \eta_{1}^{\top}\right)
$$

but it is clear that one set of data determines the other by duality.

There is a piece of Higgs field equal to zero in the following cases: 
- If $r$ is odd and $s$ even, we have the zero value only in the part corresponding to $V$ and the pair

$$
\left(V_{\frac{r+1}{2}} / V_{\frac{r-1}{2}}, Q_{V}\right)
$$

is then an orthogonal bundle with Higgs field equals to zero that appears in the decomposition of $V$, that is, an $\mathrm{SO}\left(p^{\prime}\right)$-Higgs bundle. Analogously, if $r$ is even and $s$ is odd, the pair

$$
\left(W_{\frac{s+1}{2}} / W_{\frac{s-1}{2}}, Q_{W}\right)
$$

is an $\mathrm{SO}\left(q^{\prime}\right)$-Higgs bundle in the decomposition of $W$.

- When we have $\lambda_{j} \neq \mu_{i}$ for all $1 \leq i \leq s$, then we have a pair of $\mathrm{U}\left(p^{\prime}\right)$-Higgs bundles

$$
V_{j} / V_{j-1} \text { and } V_{r-j+1} / V_{r-j}
$$

dual one to the other. Analogously, if $\mu_{i} \neq \lambda_{j}$ for all $1 \leq j \leq r$,

$$
W_{i} / W_{i-1} \text { and } W_{s-i+1} / W_{s-i}
$$

are $\mathrm{U}\left(q^{\prime}\right)$-Higgs bundle, determined one from the other by the duality given by $Q_{W}$.

Each piece in the decomposition is also polystable, and we can repeat the process and obtain a decomposition where all the pieces are stable Higgs bundles (using the JordanHölder reduction, [19, Sec. 2.10]).

Lemma 3.8. If a polystable $\mathrm{SO}_{0}(p, q)$-Higgs bundle $\left(V, Q_{V}, W, Q_{W}, \eta\right)$ decomposes as a sum of stable $G_{i}$-Higgs bundles where $G_{i}=\operatorname{SO}_{0}\left(p_{i}, q_{i}\right)$ and $\mathrm{SO}\left(n_{i}\right)$ with $n_{i} \neq 2$, then $\left(V, Q_{V}, W, Q_{W}, \eta\right)$ is stable.

If in the decomposition of a polystable $\mathrm{SO}_{0}(p, q)$-Higgs bundle $\left(V, Q_{V}, W, Q_{W}, \eta\right)$ there is a summand which is an $\mathrm{SO}(2)$-Higgs bundle, that is, a principal $\mathrm{SO}(2, \mathbb{C})$-bundle $E=$ $L \oplus L^{-1}$, the isotropic subbundles $L$ and $L^{-1}$, which have opposite degrees, do not violate the stability condition for $E$ (since there are no parabolic subgroups in $\mathrm{SO}(2, \mathbb{C})$ ) but they violate the stability condition for $\left(V, Q_{V}, W, Q_{W}, \eta\right)$.

Lemma 3.9. If an $\mathrm{SO}_{0}(p, q)$-Higgs bundle $\left(V, Q_{V}, W, Q_{W}, \eta\right)$ is strictly polystable, then in the decomposition there must be at least a $G_{i}$-Higgs bundle with $G_{i}=\mathrm{U}\left(n_{i}\right), \mathrm{SO}(2)$ or $\mathrm{U}\left(p_{i}, q_{i}\right)$. 


\subsection{Moduli space of $\mathrm{SO}_{0}(p, q)$-Higgs bundles.}

Definition 3.10. The moduli space of polystable $\mathrm{SO}_{0}(p, q)$-Higgs bundles is defined as the set of isomorphisms classes of polystable $\mathrm{SO}_{0}(p, q)$-Higgs bundles and is denoted by $\mathcal{M}\left(\mathrm{SO}_{0}(p, q)\right)$.

If $(E, \varphi)$ is an $\mathrm{SO}_{0}(p, q)$-Higgs bundle, since $E$ is the fibred product

$$
E=E_{\mathrm{SO}(p, \mathbb{C})} \times E_{\mathrm{SO}(q, \mathbb{C})}
$$

of two orthogonal bundles, we have a topological invariant $c=\left(c_{1}, c_{2}\right)$ associated to it, which is given by the following exact sequences

$$
\begin{aligned}
& 1 \rightarrow \pi_{1}(\mathrm{SO}(p, \mathbb{C})) \rightarrow \widetilde{\mathrm{SO}}(p, \mathbb{C}) \rightarrow \mathrm{SO}(p, \mathbb{C}) \rightarrow 1 \\
& 1 \rightarrow \pi_{1}(\mathrm{SO}(q, \mathbb{C})) \rightarrow \widetilde{\mathrm{SO}}(q, \mathbb{C}) \rightarrow \mathrm{SO}(q, \mathbb{C}) \rightarrow 1
\end{aligned}
$$

where $\widetilde{\mathrm{SO}}(p, \mathbb{C})$ and $\widetilde{\mathrm{SO}}(q, \mathbb{C})$ are the universal covers of $\mathrm{SO}(p, \mathbb{C})$ and $\mathrm{SO}(q, \mathbb{C})$ respectively, and the associated long cohomology sequences

$$
\begin{aligned}
& H^{1}(X, \widetilde{\mathrm{SO}}(p, \mathbb{C})) \longrightarrow H^{1}(X, \mathrm{SO}(p, \mathbb{C})) \stackrel{c_{1}}{\longrightarrow} H^{2}\left(X, \pi_{1}(\mathrm{SO}(p, \mathbb{C}))\right) \cong \pi_{1}(\mathrm{SO}(p, \mathbb{C})), \\
& H^{1}(X, \widetilde{\mathrm{SO}}(q, \mathbb{C})) \longrightarrow H^{1}(X, \mathrm{SO}(q, \mathbb{C})) \stackrel{c_{2}}{\longrightarrow} H^{2}\left(X, \pi_{1}(\mathrm{SO}(q, \mathbb{C}))\right) \cong \pi_{1}(\mathrm{SO}(q, \mathbb{C}))
\end{aligned}
$$

This invariant

$$
c=\left(c_{1}, c_{2}\right) \in \pi_{1}(\mathrm{SO}(p, \mathbb{C})) \times \pi_{1}(\mathrm{SO}(q, \mathbb{C}))
$$

measures the obstruction to lifting $E_{\mathrm{SO}(p, \mathbb{C})}$ and $E_{\mathrm{SO}(q, \mathbb{C})}$ to a flat $\left.\widetilde{\mathrm{SO}}(p, \mathbb{C})\right)$ and $\left.\widetilde{\mathrm{SO}}(p, \mathbb{C})\right)$ bundle respectively. Observe that when $n>2$, the universal cover of $\operatorname{SO}(n, \mathbb{C})$ is $\operatorname{Spin}(n, \mathbb{C})$. We have that

$$
\pi_{1}(\mathrm{SO}(n, \mathbb{C}))= \begin{cases}1, & n=1 \\ \mathbb{Z}, & n=2 \\ \mathbb{Z}_{2}, & n \geq 3\end{cases}
$$

When $p, q \geq 3$, the invariants $\left(c_{1}, c_{2}\right) \in H^{2}\left(X, \mathbb{Z}_{2}\right) \times H^{2}\left(X, \mathbb{Z}_{2}\right) \cong \mathbb{Z}_{2} \times \mathbb{Z}_{2}$ correspond to the second Stiefel-Whitney classes of the two orthogonal bundles that we obtain from the reduction of the structure groups of $E_{\mathrm{SO}(p, \mathbb{C})}$ and $E_{\mathrm{SO}(q, \mathbb{C})}$ to the real groups $\mathrm{SO}(p)$ and $\mathrm{SO}(q)$.

Since $\operatorname{det} V=\operatorname{det} W=\mathcal{O}$, using the applications

$$
H^{1}(X, \operatorname{SO}(p, \mathbb{C})), H^{1}(X, \operatorname{SO}(q, \mathbb{C})) \stackrel{\operatorname{det}}{\longrightarrow} J(X)
$$


in the Jacobian of $X$ and the identification

$$
H^{1}\left(X, \mathbb{Z}_{2}\right) \cong J_{2}(X)=\left\{L \in J(X) \mid L^{2} \cong \mathcal{O}\right\}
$$

the first Stiefel-Whitney classes of these bundles are zero.

We define the moduli space of polystable $\mathrm{SO}_{0}(p, q)$ - Higgs bundles with invariant $c$ as

$$
\mathcal{M}_{c}\left(\mathrm{SO}_{0}(p, q)\right)=\left\{(E, \varphi) \in \mathcal{M}\left(\mathrm{SO}_{0}(p, q)\right) \text { such that } c(E)=c\right\} \text {. }
$$

\section{4 $\mathrm{SO}_{0}(p, q)$ - and $\mathrm{SO}(p+q, \mathbb{C})$-Higgs bundles stability}

The goal of this section is to understand the relation between the stability of an $\mathrm{SO}_{0}(p, q)$ Higgs bundle and the stability of its associated $\mathrm{SO}(p+q, \mathbb{C})$-Higgs bundle which is defined as follows.

Let $(E, \varphi)$ be an $\mathrm{SO}_{0}(p, q)$-Higgs bundle. Extending the structure group of $E$ from $\mathrm{SO}(p, \mathbb{C}) \times \mathrm{SO}(q, \mathbb{C})$ to $\mathrm{SO}(p+q, \mathbb{C})$ and using

$$
\begin{aligned}
E_{\mathrm{SO}(p+q, \mathbb{C})}(\mathfrak{s o}(p+q, \mathbb{C})) & =E_{\mathrm{SO}(p+q, \mathbb{C})} \times_{\mathrm{Ad}} \mathfrak{s o}(p+q, \mathbb{C}) \\
& =E_{\mathrm{SO}(p, \mathbb{C}) \times \mathrm{SO}(q, \mathbb{C})}(\mathfrak{s o}(p, \mathbb{C}) \times \mathfrak{s o}(q, \mathbb{C})) \oplus E_{\mathrm{SO}(p, \mathbb{C}) \times \mathrm{SO}(q, \mathbb{C})}\left(\mathfrak{m}^{\mathbb{C}}\right),
\end{aligned}
$$

which is induced by the Cartan decomposition

$$
\mathfrak{s o}(p+q, \mathbb{C})=(\mathfrak{s o}(p, \mathbb{C}) \times \mathfrak{s o}(q, \mathbb{C})) \oplus \mathfrak{m}^{\mathbb{C}},
$$

the pair $\left(E_{\mathrm{SO}(p+q, \mathbb{C})}, \varphi\right)$, with $\varphi \in H^{0}\left(E_{\mathrm{SO}(p, \mathbb{C}) \times \mathrm{SO}(q, \mathbb{C})}\left(\mathfrak{m}^{\mathbb{C}}\right) \otimes K\right) \subset H^{0}\left(E_{\mathrm{SO}(p+q, \mathbb{C})}(\mathfrak{s o}(p+\right.$ $q, \mathbb{C})) \otimes K)$, is an $\mathrm{SO}(p+q, \mathbb{C})$-Higgs bundle.

In terms of vector bundles, if $\mathbb{E}$ is the vector bundle associated to $E_{\mathrm{SO}(p+q, \mathbb{C})}$ via the standard representation of $\mathrm{SO}(p+q, \mathbb{C})$ in $\mathbb{C}^{p+q}$ and $\left(V, Q_{V}, W, Q_{W}, \eta\right)$ is the tuple corresponding to $(E, \varphi)$, then $\mathbb{E}=V \oplus W$, and the $\mathrm{SO}(p+q, \mathbb{C})$-Higgs bundle associated to $\left(V, Q_{V}, W, Q_{W}, \eta\right)$ is the triple

$$
\left(\mathbb{E}=V \oplus W, Q=\left(\begin{array}{ll}
Q_{V} & \\
& Q_{W}
\end{array}\right), \phi=\left(\begin{array}{cc} 
& \eta \\
& -\eta^{\top}
\end{array}\right)\right) .
$$

Proposition 3.11. Let $\left(V, Q_{V}, W, Q_{W}, \eta\right)$ be an $\mathrm{SO}_{0}(p, q)$-Higgs bundle and let $(\mathbb{E}, Q, \phi)$ be the corresponding $\mathrm{SO}(p+q, \mathbb{C})$-Higgs bundle. If $\left(V, Q_{V}, W, Q_{W}, \eta\right)$ is stable, then $(\mathbb{E}, Q, \phi)$ is stable as $\mathrm{SO}(p+q, \mathbb{C})$-Higgs bundle.

Proof. Let $\left(V, Q_{V}, W, Q_{W}, \eta\right)$ be a semistable $\mathrm{SO}_{0}(p, q)$-Higgs bundle and consider the associated $\mathrm{SO}(p+q, \mathbb{C})$-Higgs bundle $(\mathbb{E}, Q, \phi)$. We will see that for every isotropic subbundle $E^{\prime} \subset \mathbb{E}$ such that $\phi\left(E^{\prime}\right) \subseteq E^{\prime}$ we have $\operatorname{deg}\left(E^{\prime}\right) \leq 0$. 
If $E^{\prime} \subset \mathbb{E}$ is an isotropic subbundle, we consider the projection $p: \mathbb{E} \rightarrow W$ and the subbundles $W^{\prime}=p\left(E^{\prime}\right)$ and $V^{\prime}=E^{\prime} \cap V$. We have the exact sequence

$$
0 \rightarrow V^{\prime} \rightarrow E^{\prime} \rightarrow W^{\prime} \rightarrow 0
$$

and the equality

$$
\operatorname{deg} E^{\prime}=\operatorname{deg} V^{\prime}+\operatorname{deg} W^{\prime} .
$$

Since $Q=\left(\begin{array}{cc}Q_{V} & \\ & Q_{W}\end{array}\right)$, we have

$$
\begin{aligned}
\left(E^{\prime}\right)^{\perp_{\mathbb{E}}} & =\left(V^{\prime} \oplus W^{\prime}\right)^{\perp_{\mathbb{E}}}=\left(V^{\prime}\right)^{\perp_{\mathbb{E}}} \cap\left(W^{\prime}\right)^{\perp_{\mathbb{E}}} \\
& =\left[\left(V^{\prime}\right)^{\perp_{V}} \oplus W\right] \cap\left[V \oplus\left(W^{\prime}\right)^{\perp_{W}}\right]=\left(V^{\prime}\right)^{\perp_{V}} \oplus\left(W^{\prime}\right)^{\perp_{W}},
\end{aligned}
$$

and then, the condition $E^{\prime} \subseteq\left(E^{\prime}\right)^{\perp_{\mathbb{E}}}$ implies $V^{\prime} \subseteq\left(V^{\prime}\right)^{\perp_{V}}$ and $W^{\prime} \subseteq\left(W^{\prime}\right)^{\perp_{W}}$, that is, $V^{\prime}$ and $W^{\prime}$ are isotropic subbundles of $V$ and $W$ respectively. On the other hand, since $\phi\left(E^{\prime}\right) \subseteq E^{\prime} \otimes K$ and $\phi=\left(\begin{array}{c}\eta \\ -\eta^{\top}\end{array}\right)$, we have $\eta\left(W^{\prime}\right) \subseteq V^{\prime} \otimes K$.

The semistability condition for $\left(V, Q_{V}, W, Q_{W}, \eta\right)$ gives $\operatorname{deg} V^{\prime}+\operatorname{deg} W^{\prime} \leq 0$ and then we conclude that semistability of an $\mathrm{SO}_{0}(p, q)$-Higgs bundle implies semistability as $\mathrm{SO}(p+$ $q, \mathbb{C})$-Higgs bundle.

Let now $E^{\prime} \subset \mathbb{E}$ to be a non-zero isotropic subbundle such that $\phi\left(E^{\prime}\right) \subseteq E^{\prime} \otimes K$. Since $E^{\prime} \neq 0$, at least one of $V^{\prime}$ and $W^{\prime}$ is non-zero, then the stability condition for $\left(V, Q_{V}, W, Q_{W}, \eta\right)$ gives $\operatorname{deg} E^{\prime}=\operatorname{deg} V^{\prime}+\operatorname{deg} W^{\prime}<0$ and we conclude.

\subsection{Smoothness and deformation theory}

It is known that a stable vector bundle is simple and that it is a smooth point of the moduli space of polystable vector bundles. On the other hand, a stable principal $\mathrm{SO}(n, \mathbb{C})$-bundle with $n \neq 2$ represents a smooth point of the moduli space $\mathcal{M}(\mathrm{SO}(n))$ if and only if it is simple (see [46]). Observe that, for $n=2$, we have $\mathrm{SO}(2, \mathbb{C}) \cong \mathbb{C}^{*}$ and then any $\mathrm{SO}(2)$ Higgs bundle is stable, simple and smooth. Thus, except in the case $n=2$, the stability of a special orthogonal bundle does not imply simplicity. In this section we study the smoothness conditions in the moduli space $\mathcal{M}\left(\mathrm{SO}_{0}(p, q)\right)$.

Definition 3.12. A G-Higgs bundle $(E, \varphi)$ is said to be simple if $\operatorname{Aut}(E, \varphi)=\operatorname{ker} \iota \cap$ $Z\left(H^{\mathbb{C}}\right)$, where $H \subset G$ is a maximal compact subgroup, $Z\left(H^{\mathbb{C}}\right)$ denotes the centre of its complexification and $\iota: H^{\mathbb{C}} \rightarrow \mathrm{GL}\left(\mathfrak{m}^{\mathbb{C}}\right)$ is the isotropy representation corresponding to the Cartan decomposition $\mathfrak{g}=\mathfrak{h}+\mathfrak{m}$ of the Lie algebra of $G$. 
A G-Higgs bundle is simple if the group of automorphisms is as small as possible. To be in ker $\iota$ means to be compatible with the Higgs field.

If $(E, Q)$ is an $\mathrm{SO}(n)$-Higgs bundle with $n>2$, that is, a principal $\mathrm{SO}(n, \mathbb{C})$-bundle, it has Higgs field equal to zero and then it is simple if and only if

$$
\operatorname{Aut}(E, Q)=Z(\operatorname{SO}(n, \mathbb{C}))= \begin{cases}I_{n}, & n \text { odd } \\ \pm I_{n}, & n \text { even }\end{cases}
$$

The group of automorphisms of an $\mathrm{SO}_{0}(p, q)$-Higgs bundle is

$$
\operatorname{Aut}\left(V, Q_{V}, W, Q_{W}, \eta\right)=\left\{(f, g) \in \operatorname{Aut}\left(V, Q_{V}\right) \times \operatorname{Aut}\left(W, Q_{W}\right) \mid \eta \circ g=f \circ \eta\right\},
$$

and hence $\left(V, Q_{V}, W, Q_{W}, \eta\right)$ is simple if

$$
\operatorname{Aut}\left(V, Q_{V}, W, Q_{W}, \eta\right)=\operatorname{ker} \iota \cap Z(\mathrm{SO}(p, \mathbb{C}) \times \mathrm{SO}(q, \mathbb{C}))
$$

The condition $f=\left(f_{1}, f_{2}\right) \in \operatorname{ker} \iota$ is equivalent to $f_{1} \circ \eta \circ f_{2}^{-1}=\eta$ and

$$
Z(\mathrm{SO}(p, \mathbb{C}) \times \mathrm{SO}(q, \mathbb{C}))= \begin{cases}I_{p+q}, & p, q \text { odd } \\ I_{p+q}, I_{p, q}, & p \text { even, } q \text { odd } \\ I_{p+q},-I_{p, q}, & p \text { odd }, q \text { even } \\ \pm I_{p+q}, \pm I_{p, q}, & p, q \text { even }\end{cases}
$$

Thus, an $\mathrm{SO}_{0}(p, q)$-Higgs bundle $\left(V, Q_{V}, W, Q_{W}, \eta\right)$ is simple if and only if

$$
\operatorname{Aut}\left(V, Q_{V}, W, Q_{W}, \eta\right)= \begin{cases} \pm I_{p+q}, & p, q \text { even } \\ I_{p+q}, & \text { rest. }\end{cases}
$$

Let us consider the deformation complex of an $\mathrm{SO}_{0}(p, q)$-Higgs bundle $(E, \varphi)$,

$$
C^{\bullet}(E, \varphi): 0 \longrightarrow E(\mathfrak{s o}(p, \mathbb{C}) \times \mathfrak{s o}(q, \mathbb{C})) \stackrel{\operatorname{ad}(\varphi)}{\longrightarrow} E\left(\mathfrak{m}^{\mathbb{C}}\right) \otimes K \longrightarrow 0
$$

We are using again the property $\left[\mathfrak{h}^{\mathbb{C}}, \mathfrak{m}^{\mathbb{C}}\right] \subseteq \mathfrak{m}^{\mathbb{C}}$ of the Cartan decomposition. For every $x \in X$ we have $\varphi_{x} \in \mathfrak{m}^{\mathbb{C}}, E\left(\mathfrak{h}^{\mathbb{C}}\right)_{x} \cong \mathfrak{h}^{\mathbb{C}}$ and

$$
\operatorname{ad}(\varphi)_{x}(H)=\left[H, \varphi_{x}\right] \in \mathfrak{m}^{\mathbb{C}} \text { for all } H \in \mathfrak{h}^{\mathbb{C}}
$$

If $\left(V, Q_{V}, W, Q_{W}, \eta\right)$ is the tuple associated to $(E, \varphi)$, we have

$$
\begin{aligned}
& C^{\bullet}\left(V, Q_{V}, W, Q_{W}, \eta\right): \mathfrak{s o}(V) \oplus \mathfrak{s o}(W) \rightarrow \operatorname{Hom}(W, V) \otimes K \\
& \left(\begin{array}{cc}
f & \\
& g
\end{array}\right) \mapsto \eta g-f \eta .
\end{aligned}
$$

We adapt the results of Biswas and Ramanan in [5] to give the following result. 
Proposition 3.13. If $(E, \varphi)$ is an $\mathrm{SO}_{0}(p, q)$-Higgs bundle, we have the following.

1. The space of endomorphisms of $(E, \varphi)$ is isomorphic to the hypercohomology group $\mathbb{H}^{0}\left(C^{\bullet}(E, \varphi)\right)$.

2. The space of infinitesimal deformations of $(E, \varphi)$ is isomorphic to the first hypercohomology group $\mathbb{H}^{1}\left(C^{\bullet}(E, \varphi)\right)$.

3. There is a long exact sequence

$$
\begin{gathered}
0 \rightarrow \mathbb{H}^{0}\left(C^{\bullet}(E, \varphi)\right) \rightarrow H^{0}\left(E\left(\mathfrak{h}^{\mathbb{C}}\right)\right) \rightarrow H^{0}\left(E\left(\mathfrak{m}^{\mathbb{C}}\right) \otimes K\right) \rightarrow \mathbb{H}^{1}\left(C^{\bullet}(E, \varphi)\right) \rightarrow \\
\rightarrow H^{1}\left(E\left(\mathfrak{h}^{\mathbb{C}}\right)\right) \rightarrow H^{1}\left(E\left(\mathfrak{m}^{\mathbb{C}}\right) \otimes K\right) \rightarrow \mathbb{H}^{2}\left(C^{\bullet}(E, \varphi)\right) \rightarrow 0,
\end{gathered}
$$

where $H^{i}\left(E\left(\mathfrak{h}^{\mathbb{C}}\right)\right) \rightarrow H^{i}\left(E\left(\mathfrak{m}^{\mathbb{C}}\right) \otimes K\right)$ is induced by $\operatorname{ad}(\varphi)$.

It follows from the above proposition that, for every $\mathrm{SO}_{0}(p, q)$-Higgs bundle $(E, \varphi)$ representing a smooth point of $\mathcal{M}$, the tangent space at this point is canonically isomorphic to $\mathbb{H}^{1}\left(C^{\bullet}(E, \varphi)\right)$.

Proposition 3.14. If an $\mathrm{SO}_{0}(p, q)$-Higgs bundle $(E, \varphi)$ is stable, simple and satisfies

$$
\mathbb{H}^{2}\left(C^{\bullet}(E, \varphi)\right)=0
$$

then it is a smooth point of the moduli space.

We say that $(E, \varphi)$ is infinitesimally simple if $\operatorname{End}(E, \varphi) \cong \mathbb{H}^{0}\left(C^{\bullet}(E, \varphi)\right)$ is isomorphic to $H^{0}(E(\operatorname{ker} d \iota \cap \mathfrak{z}))$. Stable implies infinitesimally simple.

Let $\left(V, Q_{V}, W, Q_{W}, \eta\right)$ be the tuple corresponding to $(E, \varphi)$ and consider the associated $\mathrm{SO}(p+q, \mathbb{C})$-Higgs bundle $(\mathbb{E}, Q, \phi)$ and the deformation complex

$$
C^{\bullet}(\mathbb{E}, Q, \phi): \mathfrak{s o}(\mathbb{E}) \stackrel{\operatorname{ad}(\varphi)}{\longrightarrow} \mathfrak{s o}(\mathbb{E}) \otimes K
$$

Since $\mathrm{SO}(p+q, \mathbb{C})$ is complex, infinitesimally simple in this case means $\mathbb{H}^{0}\left(C^{\bullet}(\mathbb{E}, Q, \phi)\right)=$ $0(\operatorname{ker} d \iota=\operatorname{ker}(\mathrm{ad})=0)$ and, as in the real case, stable implies infinitesimally simple. There is an isomorphism

$$
\mathbb{H}^{2}\left(C^{\bullet}(\mathbb{E}, Q, \phi)\right)=\mathbb{H}^{0}\left(C^{\bullet}(\mathbb{E}, Q, \phi)\right)^{*},
$$

and we have the following relation

$$
\mathbb{H}^{0}\left(C^{\bullet}(\mathbb{E}, Q, \phi)\right) \cong \mathbb{H}^{0}\left(C^{\bullet}(E, \varphi)\right) \oplus \mathbb{H}^{2}\left(C^{\bullet}(E, \varphi)\right)^{*}
$$


Then, if $(\mathbb{E}, Q, \phi)$ is stable, $\mathbb{H}^{0}\left(C^{\bullet}(\mathbb{E}, Q, \phi)\right)=0$ and this implies

$$
\mathbb{H}^{0}\left(C^{\bullet}(E, \varphi)\right)=\mathbb{H}^{2}\left(C^{\bullet}(E, \varphi)\right)=0
$$

Using Proposition 3.11 we obtain the following description.

Corollary 3.15. If an $\mathrm{SO}_{0}(p, q)$-Higgs bundle $(E, \varphi)$ is stable and simple, then it is a smooth point of the moduli space.

Corollary 3.16. Let $(E, \varphi)$ be a stable $\mathrm{SO}_{0}(p, q)$-Higgs bundle which represents a smooth point of $\mathcal{M}$, then

$$
\mathbb{H}^{0}\left(C^{\bullet}(E, \varphi)\right)=\mathbb{H}^{2}\left(C^{\bullet}(E, \varphi)\right)=0
$$

The expected dimension of the moduli space $\mathcal{M}\left(\mathrm{SO}_{0}(p, q)\right)$ (see [19]), is

$$
\operatorname{dim} \mathbb{H}^{1}\left(C^{\bullet}(E, \varphi)\right)=-\chi\left(C^{\bullet}(E, \varphi)\right)=\frac{n(n-1)(g-1)}{2},
$$

where $n=p+q$ and $\operatorname{dim}\left(\mathrm{SO}_{0}(p, q)\right)=\frac{n(n-1)}{2}$.

\subsection{Stable but non-simple $\mathrm{SO}(n)$ and $\mathrm{SO}_{0}(p, q)$-Higgs bundles}

In this section we give a description of the stable $\mathrm{SO}(n)$ or $\mathrm{SO}_{0}(p, q)$-Higgs bundles that fail to be simple. These results will be used in the study of strictly polystable $\mathrm{SO}_{0}(p, q)$ Higgs bundles of Section 7 .

Observe that the stability condition for a principal $\mathrm{SO}(n, \mathbb{C})$-bundle can be deduced from the stability condition for an $\mathrm{SO}(n, \mathbb{C})$-Higgs bundles given by Proposition 2.3 and Proposition 2.4 taking the Higgs field equal to zero. Of course, as in the case of $\operatorname{SO}(2, \mathbb{C})$ Higgs bundles, a principal $\mathrm{SO}(2, \mathbb{C})$-bundle is always stable.

Lemma 3.17. If an $\mathrm{SO}(n)$-Higgs bundle $(E, Q)$ decomposes as a sum of $G_{i}$-Higgs bundles and one of them is an $\mathrm{SO}(2)$-Higgs bundle or an $\mathrm{SO}\left(n_{i}\right)$-Higgs bundle with $n>2$ which is not stable, then $(E, Q)$ is not stable.

Proof. If there is a summand which is an $\mathrm{SO}(2)$-Higgs bundle $E_{i}=L \oplus L^{-1}$, the isotropic subbundles $L$ and $L^{-1}$, which have opposite degrees, do not violate the stability condition for $E$ but they violate the stability condition for $(E, Q)$. If a summand $\left(E_{i}, Q_{i}\right)$ is a non-stable $\mathrm{SO}\left(n_{i}\right)$-Higgs bundle, there is a proper isotropic subbundle $F_{i} \subset E_{i}$ such that $\operatorname{deg} F_{i} \geq 0$. Since $Q_{i}$ is the restriction of $Q$ to $E_{i}, F_{i}$ is an isotropic subbundle of $E$ that violates its stability. 
Theorem 3.18. Let $(E, Q)$ be a stable $\mathrm{SO}(n)$-Higgs bundle with $n \neq 2$, that is, a principal $\mathrm{SO}(n, \mathbb{C})$-bundle, which is not simple, then it decomposes as a sum of stable and simple $\mathrm{SO}\left(n_{i}\right)$-Higgs bundles with $n_{i} \neq 2$.

Proof. Since $(E, Q)$ is not simple and

$$
Z(\mathrm{SO}(n, \mathbb{C}))= \begin{cases}I_{n}, & n \text { odd } \\ \pm I_{n}, & n \text { even, }\end{cases}
$$

there is an automorphism $f \in \operatorname{Aut}(E, Q) \backslash\left\{ \pm I_{n}\right\}$ if $n$ even, or $f \in \operatorname{Aut}(E, \varphi) \backslash\left\{I_{n}\right\}$ if $n$ is odd.

Suppose that $f=\lambda I_{n}$ with $\lambda \in \mathbb{C}^{*}$. It has to preserve the orthogonal structure of $E$, that is,

$$
Q\left(f(e), f\left(e^{\prime}\right)\right)=\lambda^{2} Q\left(e, e^{\prime}\right)=Q\left(e, e^{\prime}\right),
$$

and this happens if and only if $\lambda= \pm 1$. On the other hand, the determinant of $f$ has to be equal to one. Then, the only possibilities are $f= \pm I_{n}$ if $n$ is even and $f=I_{n}$ if $n$ is odd, which are exactly the cases that we are excluding.

The group $\operatorname{Aut}(E, \varphi)$ is reductive. This implies that $f$ may be chosen in such a way that there is a splitting $E=\bigoplus E_{i}$ such that $f$ restricted to $E_{i}$ is $\lambda_{i} I_{n}$ with $\lambda_{i} \in \mathbb{C}^{*}$.

Since

$$
Q\left(e_{i}, e_{j}\right)=Q\left(f\left(e_{i}\right), f\left(e_{j}\right)\right)=\lambda_{i} \lambda_{j} Q\left(e_{i}, e_{j}\right),
$$

then $Q\left(E_{i}, E_{j}\right)$ can only be non-zero when $\lambda_{i} \lambda_{j}=1$. Since $Q$ is non-degenerate, the possible $\lambda^{\prime} s$ values of lambda come in pairs $\left(\lambda_{i}, \lambda_{i}^{-1}\right)$ corresponding to $\left(E_{i}, E_{i}^{*}\right)$. If $\lambda_{i}= \pm 1$, we have $\lambda_{i}=\lambda_{i}^{-1}$ and then $E_{1} \cong E_{1}^{*}$ and $E_{-1} \cong E_{-1}^{*}$. Since $\operatorname{det} f=\prod_{i} \lambda_{i}^{\text {rk } E_{i}}=1$, we do not have the value $\lambda_{i}=0$.

Suppose that there is a $\lambda_{i} \neq \pm 1$, then $E_{i} \subset E$ is an isotropic subbundle of $E$. If $\operatorname{deg} E_{i} \geq 0$, this subbundle violates the stability condition for $(E, Q)$. If $\operatorname{deg} E_{i}<0$, then $\operatorname{deg} E_{i}^{*}>0$ and again $(E, Q)$ is not stable. Hence $\lambda_{i}= \pm 1$ and $(E, Q)=\left(E_{1}, Q_{1}\right) \oplus$ $\left(E_{-1}, Q_{-1}\right)$.

Lemma 3.17 tell us that these summands are stable $\mathrm{SO}\left(n_{i}\right)$-Higgs bundles with $n_{i} \neq 2$.

If there is a summand which is a non-simple $\mathrm{SO}\left(n_{i}\right)$-Higgs bundle, applying the argument of this proof inductively we conclude that a stable but non-simple $\mathrm{SO}(n)$-Higgs bundle can be decomposed as a sum of smooth $\mathrm{SO}\left(n_{i}\right)$-Higgs bundles.

Lemma 3.19. If an $\mathrm{SO}_{0}(p, q)$-Higgs bundle $\left(V, Q_{V}, W, Q_{W}, \eta\right)$ decomposes as a sum of $G_{i^{-}}$ Higgs bundles and one of them is an $\mathrm{SO}_{0}\left(p_{i}, q_{i}\right)$-Higgs bundle $\left(V_{i}, Q_{V}, W_{i}, Q_{W}, \eta_{i}\right)$ which is not stable, then $\left(V, Q_{V}, W, Q_{W}, \eta\right)$ is not stable. 
Proof. Since $\left(V_{i}, Q_{V}, W_{i}, Q_{W}, \eta_{i}\right)$ is not stable, there are isotropic subbundles $V^{\prime} \subset V_{i}$, $W^{\prime} \subset W_{i}$ such that $\eta_{i}\left(W^{\prime}\right) \subseteq V^{\prime} \otimes K$ and with $\operatorname{deg} V^{\prime}+\operatorname{deg} W^{\prime} \geq 0$. These are also isotropic subbundles of $V$ and $W$ and violate the stability condition for $\left(V, Q_{V}, W, Q_{W}, \eta\right)$.

Lemma 3.20. If an $\mathrm{SO}_{0}(p, q)$-Higgs bundle $\left(V, Q_{V}, W, Q_{W}, \eta\right)$ decomposes as a sum of $G_{i}$-Higgs bundles and one of them is an $\mathrm{SO}(2)$-Higgs bundle or an $\mathrm{SO}\left(n_{i}\right)$-Higgs bundle which is not stable, then $\left(V, Q_{V}, W, Q_{W}, \eta\right)$ is not stable.

Proof. It can be deduced from the proof of Lemma 3.17 and Lemma 3.19.

Theorem 3.21. Let $\left(V, Q_{V}, W, Q_{W}, \eta\right)$ be a stable $\mathrm{SO}_{0}(p, q)$-Higgs bundle which is not simple, then it decomposes as a sum of stable and simple $\mathrm{SO}_{0}\left(p_{i}, q_{i}\right)$ and $\mathrm{SO}\left(n_{i}\right)$-Higgs bundles with $n_{i} \neq 2$.

Proof. Suppose that the Higgs field is equal to zero, then the $\mathrm{SO}_{0}(p, q)$-Higgs bundle $\left(V, Q_{V}, W, Q_{W}, \eta\right)$ is a stable principal $\mathrm{SO}(p, \mathbb{C}) \times \mathrm{SO}(q, \mathbb{C})$-bundle, that is, it decomposes as a sum of a stable $\mathrm{SO}(p)$-Higgs bundle and a stable $\mathrm{SO}(q)$-Higgs bundle. If the summands are simple we have the result and if they are not, we conclude using Theorem 3.18 .

Suppose now that $\eta \neq 0$. Since $\left(V, Q_{V}, W, Q_{W}, \eta\right)$ is not simple, there is an automorphism $f \in \operatorname{Aut}\left(V, Q_{V}, W, Q_{W}, \eta\right) \backslash\left\{ \pm I_{p+q}\right\}$ if $p$ and $q$ are even, or an automorphism $f \in \operatorname{Aut}\left(V, Q_{V}, W, Q_{W}, \eta\right) \backslash\left\{I_{p+q}\right\}$ in the rest of the cases.

Suppose that $f=\left(f_{1}, f_{2}\right)=\left(\lambda I_{p}, \mu I_{q}\right)$ is a multiple of the identity in $V$ and $W$ $\left(\lambda, \mu \in \mathbb{C}^{*}\right)$. The determinant of $f_{1}$ and $f_{2}$ has to be equal to 1 and they have to preserve the corresponding orthogonal structures, that is,

$$
\begin{gathered}
Q_{V}\left(f_{1}(v), f_{1}\left(v^{\prime}\right)\right)=\lambda^{2} Q_{V}\left(v, v^{\prime}\right)=Q_{V}\left(v, v^{\prime}\right), \\
Q_{W}\left(f_{2}(w), f_{2}\left(w^{\prime}\right)\right)=\mu^{2} Q_{W}\left(w, w^{\prime}\right)=Q_{W}\left(w, w^{\prime}\right) .
\end{gathered}
$$

On the other hand, since we are supposing that the automorphisms are multiples of the identity, the condition $f_{1} \circ \eta=\eta \circ f_{2}$ is equivalent to $f_{1}=f_{2}$. Then, the only possibilities are $f= \pm I_{p+q}$ if $p$ and $q$ are even and $f=I_{p+q}$ in the rest of the cases, which are exactly the cases that we are excluding. Thus, $f$ is not of this form. Observe that it is possible to have one of the automorphisms, $f_{1}$ or $f_{2}$, equal to a multiple of the identity, more exactly, the identity when the rank ( $p$ or $q$ ) is odd and \pm 1 when the rank is even.

Since the group $\operatorname{Aut}\left(V, Q_{V}, W, Q_{W}, \eta\right)$ is reductive, there are splittings $V=\bigoplus V_{j}$, $W=\bigoplus W_{i}$ such that $f_{1}$ and $f_{2}$ are $\lambda_{j}$ and $\mu_{i}$ in $V_{j}$ and $W_{i}$ respectively $\left(\lambda_{j}, \mu_{i} \in \mathbb{C}^{*}\right)$. 
Since

$$
Q_{V}\left(v_{i}, v_{j}\right)=Q_{V}\left(f_{1}\left(v_{i}\right), f_{1}\left(v_{j}\right)\right)=\lambda_{i} \lambda_{j} Q_{V}\left(v_{i}, v_{j}\right)
$$

then $Q_{V}\left(V_{i}, V_{j}\right)$ can only be non-zero when $\lambda_{i} \lambda_{j}=1$. Since $Q_{V}$ is non-degenerate, the possible values of lambda come in pairs $\left(\lambda_{j}, \lambda_{j}^{-1}\right)$ corresponding to $\left(V_{j}, V_{j}^{*}\right)$. If $\lambda_{j}= \pm 1$, we have $\lambda_{j}=\lambda_{j}^{-1}$ and then $V_{1} \cong V_{1}^{*}$ and $V_{-1} \cong V_{-1}^{*}$. Since $\operatorname{det} f_{1}=\prod_{j} \lambda_{j}^{\mathrm{rk} V_{j}}=1$, we do not have $\lambda_{j}=0$. The same for $\left(W, Q_{W}\right)$.

Since $f$ preserve the Higgs field, for each component $\eta_{i, j} \in H^{0}\left(\operatorname{Hom}\left(W_{i}, V_{j}\right) \otimes K\right)$, we have that

$$
\eta_{i, j}\left(f_{2}(w)\right)=\mu_{i} \eta_{i, j}(w)
$$

is equal to

$$
f_{1}\left(\eta_{i, j}(w)\right)=\lambda_{j} \eta_{i, j}(w)
$$

for all $w \in W_{i}$, and then, $\mu_{i} \neq \lambda_{j}$ implies $\eta_{i, j}=0$.

Suppose that there is a $\lambda_{j} \neq \pm 1$. If $\mu_{i} \neq \lambda_{j}$ for all $i$, we have $\eta_{i, j}=0$ for all $i$, that is, $\eta^{\top}\left(V_{j}\right)=0$. Since

$$
Q_{V}\left(V_{j}, V_{j}\right)=Q_{V}\left(f_{1}\left(V_{j}\right), f_{1}\left(V_{j}\right)\right)=\lambda_{j}^{2} Q_{V}\left(V_{j}, V_{j}\right)
$$

and $\lambda_{j}^{2} \neq 1$, we have $Q_{V}\left(V_{j}, V_{j}\right)=0$ and hence, $V_{j} \subset V$ is an isotropic subbundle. If $\operatorname{deg} V_{j} \geq 0$, this subbundle violates the stability condition for $\left(V, Q_{V}, W, Q_{W}, \eta\right)$. If $\operatorname{deg} V_{j}<0$, then $\operatorname{deg} V_{j}^{*}>0$ and again $\left(V, Q_{V}, W, Q_{W}, \eta\right)$ is not stable and we get a contradiction. Analogously, if there is a $\mu_{i} \neq \pm 1$ with $\lambda_{j} \neq \mu_{i}$ for all $j$, we obtain a contradiction with the stability of $\left(V, Q_{V}, W, Q_{W}, \eta\right)$. Finally, suppose that there is a $\lambda_{i} \neq \pm 1$ such that $\lambda_{j}=\mu_{i}$ for some $i$. The subbundles $V_{j} \subset V$ and $W_{i} \subset W$ are isotropic and $\eta\left(W_{i}\right) \subseteq V_{j} \otimes K$. If $\operatorname{deg} V_{j}+\operatorname{deg} W_{i} \geq 0$, these subbundles violate the stability condition for $\left(V, Q_{V}, W, Q_{W}, \eta\right)$. If $\operatorname{deg} V_{i}+\operatorname{deg} W_{i}<0$, then $\operatorname{deg} V_{i}^{*}+\operatorname{deg} W_{i}^{*}>0$ and again $\left(V, Q_{V}, W, Q_{W}, \eta\right)$ is not stable. Then $\lambda_{j}, \mu_{i}= \pm 1$.

Since $1=\operatorname{det} f_{1}=1^{\mathrm{rk} V_{1}} \cdot(-1)^{\mathrm{rk} V_{-1}}$ and analogously for $f_{2}$, we have $\mathrm{rk} V_{-1}$ and $\mathrm{rk} W_{-1}$ even.

Denote $\eta_{i}=\eta_{i, i}$, where $i= \pm 1$. We have the following decomposition

$$
\left(V, Q_{V}, W, Q_{W}, \eta\right)=\left(V_{1}, W_{1}, \eta_{1}\right) \oplus\left(V_{-1}, W_{-1}, \eta_{-1}\right)
$$

Since at least one piece of the automorphism, $f_{1}$ or $f_{2}$, is not a multiple of the identity, only one of the four subbundles $V_{1}, V_{-1}, W_{1}$ and $W_{-1}$ can be zero. Since $\eta \neq 0$, at least one of the Higgs fields $\eta_{1}$ and $\eta_{-1}$ has to be non-zero. Thus, $\left(V, Q_{V}, W, Q_{W}, \eta\right)$ is a sum 
of two $\mathrm{SO}_{0}\left(p_{i}, q_{i}\right)$-Higgs bundles, $\left(V_{1}, W_{1}, \eta_{1}\right)$ and $\left(V_{-1}, W_{-1}, \eta_{-1}\right)$, or it is a sum of an $\mathrm{SO}_{0}\left(p_{i}, q_{i}\right)$-Higgs bundle together with one or two $\mathrm{SO}\left(n_{i}\right)$-Higgs bundles.

Lemma 3.19 and Lemma 3.20 tell us that these summands are stable $G_{i}$-Higgs bundles.

If there is a summand which is a non-simple $\mathrm{SO}\left(n_{i}\right)$-Higgs bundle, we have from Theorem 3.18 that it decomposes as a sum of stable and simple orthogonal bundles. If there is a summand which is a non-simple $\mathrm{SO}_{0}\left(p_{i}, q_{i}\right)$-Higgs bundle, applying the argument of this proof inductively we conclude that it can be decomposed as a sum of stable and simple $G_{i}$-Higgs bundles with $G_{i}=\mathrm{SO}_{0}\left(p_{i}, q_{i}\right)$ and $\mathrm{SO}\left(n_{i}\right)$.

Let $\left(V, Q_{V}, W, Q_{W}, \eta\right)$ be a stable but non-simple $\mathrm{SO}_{0}(p, q)$-Higgs bundle and consider the decomposition given by Theorem 3.21. Since all the summands are smooth, they are simple, and since $\left(V, Q_{V}, W, Q_{W}, \eta\right)$ is not simple, it must have at least one summand of the following types: a smooth $\mathrm{SO}_{0}\left(p_{i}, q_{i}\right)$-Higgs bundle with $p_{i}$ and $q_{i}$ even, or a smooth $\mathrm{SO}\left(n_{i}\right)$-Higgs bundle with $n_{i}$ even. These conditions allow us to take the automorphism -1 in this summand and guarantee the non-simplicity. 



\section{$4 \quad \mathrm{SO}_{0}(p, q)$-Higgs bundles and surface group represen- tations}

Let $S$ be a compact oriented smooth surface of genus $g$ and let $\pi_{1}$ be its fundamental group. The group $\mathrm{SO}_{0}(p, q)$ acts on the set $\operatorname{Hom}\left(\pi_{1}, \mathrm{SO}_{0}(p, q)\right)$ of representations of the fundamental group in $\mathrm{SO}_{0}(p, q)$ by conjugation:

$$
(g \cdot \rho)(\gamma)=g \rho(\gamma) g^{-1}
$$

for $g \in \mathrm{SO}_{0}(p, q), \rho \in \operatorname{Hom}\left(\pi_{1}, \mathrm{SO}_{0}(p, q)\right)$ and $\gamma \in \pi_{1}$.

A representation $\rho \in \operatorname{Hom}\left(\pi_{1}, \mathrm{SO}_{0}(p, q)\right)$ is said to be reductive if the composition with the adjoint representation in the Lie algebra

$$
\pi_{1} \stackrel{\rho}{\longrightarrow} \mathrm{SO}_{0}(p, q) \stackrel{\mathrm{Ad}}{\longrightarrow} \mathrm{GL}(\mathfrak{s o}(p, q))
$$

decomposes as a sum of irreducible representations. We will denote the subspace of all reductive representations by $\mathrm{Hom}^{+}\left(\pi_{1}, \mathrm{SO}_{0}(p, q)\right)$.

Definition 4.1. The moduli space of representations of $\pi_{1}$ in $\mathrm{SO}_{0}(p, q)$ is defined as the orbit space

$$
\mathcal{R}\left(\mathrm{SO}_{0}(p, q)\right)=\operatorname{Hom}^{+}\left(\pi_{1}, \mathrm{SO}_{0}(p, q)\right) / \mathrm{SO}_{0}(p, q)
$$

Consider the usual presentation

$$
\pi_{1}=\left\langle a_{1}, b_{1}, \ldots, a_{g}, b_{g} \mid \prod_{i=1}^{g}\left[a_{i}, b_{i}\right]=1\right\rangle,
$$

where $\left[a_{i}, b_{i}\right]=a_{i} b_{i} a_{i}^{-1} b_{i}^{-1}$. Every representation is determined by the images of the generators $a_{1}, b_{1}, \ldots, a_{g}, b_{g}$. Then, $\operatorname{Hom}\left(\pi_{1}, \mathrm{SO}_{0}(p, q)\right)$ can be embedded in $\mathrm{SO}_{0}(p, q)^{2 g}$ via

$$
\rho \mapsto\left(\rho\left(a_{1}\right), \ldots, \rho\left(b_{g}\right)\right) \in \mathrm{SO}_{0}(p, q)^{2 g},
$$

and can be identified with the subset of $\mathrm{SO}_{0}(p, q)^{2 g}$ of elements $\left(A_{1}, B_{1}, \ldots, A_{g}, B_{g}\right)$ satisfying the algebraic equation $\prod_{i=1}^{g}\left[A_{i}, B_{i}\right]=1$. Thus, $\operatorname{Hom}\left(\pi_{1}, \mathrm{SO}_{0}(p, q)\right)$ have structure of real analytic variety. The set of reductive representations $\operatorname{Hom}^{+}\left(\pi_{1}, \mathrm{SO}_{0}(p, q)\right)$ is an open subset, and then has also structure of real analytic variety. Moreover, the moduli space $\mathcal{R}\left(\mathrm{SO}_{0}(p, q)\right)$ is also a real analytic variety and it is Hausdorff with the quotient topology. (See Goldman [22]). 


\subsection{Representations and flat connections}

There is a correspondence between the representations of the fundamental group on $\mathrm{SO}_{0}(p, q)$, modulo the action of $\mathrm{SO}_{0}(p, q)$, and the set of equivalence classes of flat principal $\mathrm{SO}_{0}(p, q)$-bundles. (See [35], Chap. I, §2).

To any representation $\rho: \pi_{1} \rightarrow \mathrm{SO}_{0}(p, q)$, we can associate a flat principal $\mathrm{SO}_{0}(p, q)$ bundle

$$
E_{\rho}=\tilde{S} \times{ }_{\rho} \mathrm{SO}_{0}(p, q),
$$

where $\tilde{S}$ is the universal cover of $S$, which is a flat principal $\pi_{1}$-bundle. This is the orbit space for the action of $\pi_{1}$ on $\tilde{S}$ and the action

$$
a \cdot A=\rho(a) A \rho(a)^{-1}
$$

of $\pi_{1}$ on $\mathrm{SO}_{0}(p, q)$ via $\rho$.

Conversely, let $(E, D)$ be a flat principal $\mathrm{SO}_{0}(p, q)$-bundle. If $\gamma=\gamma(t)$ with $0 \leq t \leq a$ is a curve in $S$ and $\xi$ is a section of $E$ defined along $\gamma$, the section $\xi$ is parallel along $\gamma$ if

$$
D \xi\left(\gamma^{\prime}(t)\right)=0 \text { for } 0 \leq t \leq a,
$$

where $\gamma^{\prime}(t)$ is the tangent vector of $\gamma$ at $\gamma(t)$. If $\xi_{0}$ is in the fibre $E_{\gamma(0)}$, it extends uniquely to a parallel section $\xi$ along $\gamma$ called the parallel displacement of $\xi_{0}$ and this induces a linear transformation of the fibre $E_{x_{0}}$, where $x_{0}=\gamma(0)=\gamma(a)$. Since $D$ is flat, the parallel displacement depends only on the homotopy class of $\gamma$ and then we have a representation

$$
\rho_{(E, D)}: \pi_{1} \rightarrow \mathrm{SO}_{0}(p, q)
$$

called the holonomy representation of $E$.

We say that a flat connection $D$ in a principal $\mathrm{SO}_{0}(p, q)$-bundle $E$ is reductive if the representation of $\pi_{1}$ in $\mathrm{SO}_{0}(p, q)$ corresponding to $(E, D)$ is reductive. Then, if we restrict to reductive elements, the correspondence between representations of the fundamental group on $\mathrm{SO}_{0}(p, q)$ modulo the action of $\mathrm{SO}_{0}(p, q)$ and the set of equivalence classes of flat principal $\mathrm{SO}_{0}(p, q)$-bundles gives a correspondence

$$
\mathcal{R}\left(\mathrm{SO}_{0}(p, q)\right) \cong \mathcal{F}\left(\mathrm{SO}_{0}(p, q)\right)
$$

being

$$
\begin{aligned}
\mathcal{F}\left(\mathrm{SO}_{0}(p, q)\right)= & \{\text { reductive flat connections } D \text { on a principal } \\
& \left.\mathrm{SO}_{0}(p, q) \text {-bundle } E\right\} / \mathcal{G}(E),
\end{aligned}
$$


where $\mathcal{G}(E)$ denotes the gauge group of $E$, that is, the group of $\mathrm{SO}_{0}(p, q)$-invariant automorphisms of $E$ and the action of the gauge group on the space of connections is given by

$$
g \cdot D=g \circ D \circ g^{-1}
$$

which preserves the condition of flatness.

The maximal compact subgroup of $\mathrm{SO}(p, q)$ is $\mathrm{S}(\mathrm{O}(p) \times \mathrm{O}(q))$, while $\mathrm{SO}(p) \times \mathrm{SO}(q)$ is the maximal compact subgroup of $\mathrm{SO}_{0}(p, q)$. The fundamental group of $\mathrm{SO}_{0}(p, q)$, coincides with the fundamental group of its maximal compact subgroup. Using

$$
\begin{aligned}
& \pi_{1}(\mathrm{SO}(1))=1, \text { since } \mathrm{SO}(1)=\{1\} \\
& \pi_{1}(\mathrm{SO}(2))=\mathbb{Z}, \text { since } \mathrm{SO}(2) \cong S^{1}, \\
& \pi_{1}(\mathrm{SO}(n))=\mathbb{Z}_{2}, \text { for } n>2,
\end{aligned}
$$

we obtain

$$
\begin{array}{c|ccc}
\pi_{1}\left(\mathrm{SO}_{0}(p, q)\right) & p=1 & p=2 & p \geq 3 \\
\hline q=1 & 1 & \mathbb{Z} & \mathbb{Z}_{2} \\
q=2 & \mathbb{Z} & \mathbb{Z} \times \mathbb{Z} & \mathbb{Z}_{2} \times \mathbb{Z} \\
q \geq 3 & \mathbb{Z}_{2} & \mathbb{Z} \times \mathbb{Z}_{2} & \mathbb{Z}_{2} \times \mathbb{Z}_{2}
\end{array}
$$

Let us consider the following exact sequence

$$
1 \rightarrow \pi_{1}\left(\mathrm{SO}_{0}(p, q)\right) \rightarrow \widetilde{\mathrm{SO}_{0}}(p, q) \rightarrow \mathrm{SO}_{0}(p, q) \rightarrow 1
$$

where $\widetilde{\mathrm{SO}}_{0}(p, q)$ is the universal cover of $\mathrm{SO}_{0}(p, q)$, which coincides with the Lie group $\operatorname{Spin}_{0}(p, q)$ when $p, q>2$. The associated long cohomology sequence is

$$
H^{1}\left(S, \widetilde{\mathrm{SO}}_{0}(p, q)\right) \longrightarrow H^{1}\left(S, \mathrm{SO}_{0}(p, q)\right) \stackrel{c_{2}}{\longrightarrow} H^{2}\left(S, \pi_{1}\left(\mathrm{SO}_{0}(p, q)\right)\right) \cong \pi_{1}\left(\mathrm{SO}_{0}(p, q)\right),
$$

where we are regarding $\widetilde{\mathrm{SO}}_{0}(p, q), \mathrm{SO}_{0}(p, q)$ and $\pi_{1}\left(\mathrm{SO}_{0}(p, q)\right)$ as the sheafs of locally constant functions.

The sets $H^{1}\left(S, \widetilde{\mathrm{SO}}_{0}(p, q)\right)$ and $H^{1}\left(S, \mathrm{SO}_{0}(p, q)\right)$ parameterize the set of equivalence classes of flat principal bundles over $S$ with structure group $\widetilde{\mathrm{SO}_{0}}(p, q)$ and $\mathrm{SO}_{0}(p, q)$ respectively. The characteristic class

$$
c\left(E_{\rho}\right) \in H^{2}\left(S, \pi_{1}\left(\mathrm{SO}_{0}(p, q)\right)\right) \cong \pi_{1}\left(\mathrm{SO}_{0}(p, q)\right)
$$

measures the obstruction to lifting $E_{\rho}$ to a flat $\widetilde{\mathrm{SO}_{0}}(p, q)$-bundle or equivalently to lifting $\rho$ to a representation of $\pi_{1}$ in $\widetilde{\mathrm{SO}}_{0}(p, q)$. This class is an invariant for the classification of 
principal $\mathrm{SO}_{0}(p, q)$-bundles on $S$, and then it is an invariant for the representations of $\pi_{1}$ in $\mathrm{SO}_{0}(p, q)$. As we have seen, for $p+q \geq 3$, this invariant can be an integer, an element of the group $\mathbb{Z}_{2}$, a pair of integers, a pair in $\mathbb{Z}_{2} \times \mathbb{Z}_{2}$ or in $\mathbb{Z}_{2} \times \mathbb{Z}$, depending on the values of $p$ and $q$. The number of liftings is measured by $H^{1}\left(S, \pi_{1}\left(\mathrm{SO}_{0}(p, q)\right)\right)$.

The moduli space of reductive representations of $\pi_{1}$ in $\mathrm{SO}_{0}(p, q)$ with invariant $c$ is defined as

$$
\mathcal{R}_{c}\left(\mathrm{SO}_{0}(p, q)\right)=\left\{\rho \in \mathcal{R}\left(\mathrm{SO}_{0}(p, q)\right) \text { such that } c(\rho)=c\right\} .
$$

Since $c$ is constant on each connected component of $\mathcal{R}\left(\mathrm{SO}_{0}(p, q)\right)$, then $\mathcal{R}_{c}\left(\mathrm{SO}_{0}(p, q)\right)$ is a connected component or a union of connected components. Fixing the invariant $c$ we have the correspondence

$$
\mathcal{R}_{c}\left(\mathrm{SO}_{0}(p, q)\right) \cong \mathcal{F}_{c}\left(\mathrm{SO}_{0}(p, q)\right)
$$

where

$$
\begin{gathered}
\mathcal{F}_{c}\left(\mathrm{SO}_{0}(p, q)\right)=\left\{\text { reductive flat connections } D \text { on a principal } \mathrm{SO}_{0}(p, q)\right. \text {-bundle } \\
E \text { with } c(E)=c\} / \mathcal{G}(E) .
\end{gathered}
$$

\subsection{Harmonic reductions and harmonic equations}

Let $E$ be a principal $\mathrm{SO}_{0}(p, q)$-bundle over $S$. This bundle admits a reduction of the structure group from $\mathrm{SO}_{0}(p, q)$ to the maximal compact $\mathrm{SO}(p) \times \mathrm{SO}(q)$ if there are a principal $\mathrm{SO}(p) \times \mathrm{SO}(q)$-bundle $F$ and an injection $F \subset E$ such that

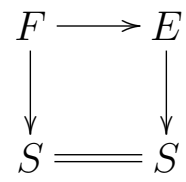

is commutative. This gives a decomposition

$$
E=F \times_{\mathrm{SO}(p) \times \mathrm{SO}(q)} \mathrm{SO}_{0}(p, q) .
$$

A reduction of structure group is equivalent to giving a section $h$ of the bundle

$$
E_{\mathrm{SO}_{0}(p, q) / \mathrm{SO}(p) \times \mathrm{SO}(q)}=E \times \times_{\mathrm{SO}_{0}(p, q)}\left(\mathrm{SO}_{0}(p, q) / \mathrm{SO}(p) \times \mathrm{SO}(q)\right),
$$

which is obtained by considering the transitive action of $\mathrm{SO}_{0}(p, q)$ on the symmetric space $\mathrm{SO}_{0}(p, q) / \mathrm{SO}(p) \times \mathrm{SO}(q)$. Lifting to the universal cover $\tilde{S}$ of $S$, we have

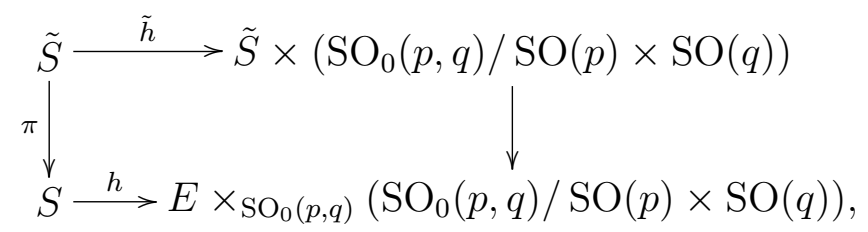


and then, the reduction is now a section of $\tilde{S} \times\left(\mathrm{SO}_{0}(p, q) / \mathrm{SO}(p) \times \mathrm{SO}(q)\right)$, or equivalently a map

$$
\tilde{h}: \tilde{S} \rightarrow \mathrm{SO}_{0}(p, q) / \mathrm{SO}(p) \times \mathrm{SO}(q)
$$

equivariant under the action of $\pi_{1}$.

Let $X$ be a compact Riemannian manifold and $Y$ a compact Riemannian manifold with non-positive sectional curvature.

Definition 4.2. If $f: X \rightarrow Y$ is a smooth map, we define the energy density of $f$ as

$$
\begin{aligned}
e(f): X & \longrightarrow \mathbb{R}^{+} \\
x & \longmapsto \operatorname{Tr}\left(d f_{x}^{*} d f_{x}\right),
\end{aligned}
$$

where $d f_{x}^{*} d f_{x}: T_{x} X \rightarrow T_{x} X$, and the energy of $f$ as the integral

$$
E(f)=\int_{X} e(f) d \nu_{X}
$$

where $d \nu_{X}$ is the volume element in $X$.

The Laplace operator applied to $f$ is

$$
\Delta f=\operatorname{div}(\nabla f)=d^{*} d f
$$

Definition 4.3. The map $f: X \rightarrow Y$ is harmonic if $\Delta f=0$.

This condition is related to the Euler-Lagrange equation for the energy functional. The harmonic maps are in fact critical points of the energy functional (see [16]).

Using this definition, the map $\tilde{h}$ is harmonic if

$$
\Delta \tilde{h}=d^{*} d \tilde{h}=0
$$

There is another notion of harmonicity for a reduction of structure group that we will describe next. We will see that both are equivalent.

Let $P$ be a principal $G$-bundle and consider the gauge group $\mathcal{G}(P)$ of $G$-invariant automorphisms of $P$. The sections of the associated bundle of groups

$$
P(G)=P \times_{G} G,
$$


where $G$ acts on $G$ by internal automorphisms, are equivalent to maps $h: P \rightarrow G$ satisfying

$$
h(p g)=g^{-1} h(p) g,
$$

and forms a group that can be identified with the gauge group $\mathcal{G}(P)$. The correspondence is given by

$$
\begin{aligned}
\Omega^{0}(P(G)) & \leftrightarrow \mathcal{G}(P) \\
h & \leftrightarrow h_{*}: P \rightarrow P, h_{*}(p)=p h(p)
\end{aligned}
$$

The $\operatorname{map} h_{*}$ is equivariant:

$$
h_{*}(p g)=p g h(p g)=p g g^{-1} h(p) g=h_{*}(p) g .
$$

The Lie algebra of the gauge group $\mathcal{G}(P)$ can be described as well as the set of holomorphic sections of

$$
P(\mathfrak{g})=P \times_{\text {Ad }} \mathfrak{g} .
$$

Then, $\mathcal{G}(P)=\Omega^{0}(P(G))$ and Lie $\mathcal{G}(P)=\Omega^{0}(P(\mathfrak{g}))$.

Applying this to our case, we have $\mathcal{G}(F)=\Omega^{0}(F(\mathrm{SO}(p) \times \mathrm{SO}(q)))$ and Lie $\mathcal{G}(F)=$ $\Omega^{0}(F(\mathfrak{s o}(p) \times \mathfrak{s o}(q)))$, that is, if we consider $F$ as the fibred product of two principal bundles with structure groups $\mathrm{SO}(p)$ and $\mathrm{SO}(q)$ respectively,

$$
F=E_{\mathrm{SO}(p)} \times E_{\mathrm{SO}(q)},
$$

a gauge transformation $g \in \mathcal{G}(F)$ is a pair $\left(g_{1}, g_{2}\right)$ of sections $g_{1} \in \Omega^{0}\left(E_{\mathrm{SO}(p)}(\mathrm{SO}(p))\right)$ and $g_{2} \in \Omega^{0}\left(E_{\mathrm{SO}(q)}(\mathrm{SO}(q))\right)$.

A reduction of the structure group from $\mathrm{SO}_{0}(p, q)$ to the maximal compact $\mathrm{SO}(p) \times$ $\mathrm{SO}(q)$ gives, for any connection $D$ on the principal $\mathrm{SO}_{0}(p, q)$-bundle $E$, a unique splitting

$$
D=A+\psi
$$

where $A$ is a connection on $F$ and $\psi$ is in $\Omega^{1}(F(\mathfrak{m}))$, where we use $F(\mathfrak{m})$ to denote the bundle associated to $F$ via the isotropy representation.

There is an action of the gauge group $\mathcal{G}(F)$ on the set

$$
\left\{(A, \psi) \mid A \text { connection on } F, \psi \in \Omega^{1}(F(\mathfrak{m}))\right\} .
$$

A connection $A$ on the principal bundle $F$ is equivalent to a pair of connections $\left(A_{1}, A_{2}\right)$ on the two principal bundles $E_{\mathrm{SO}(p)}$ and $E_{\mathrm{SO}(q)}$. Let $\omega_{1}$ and $\omega_{2}$ be the corresponding connections 1-forms defined by

$$
\begin{aligned}
\left(\omega_{1}\right)_{p}: T_{p} E_{\mathrm{SO}(p)} & \rightarrow \mathfrak{s o}(p) & \left(\omega_{2}\right)_{q}: T_{q} E_{\mathrm{SO}(q)} & \rightarrow \mathfrak{s o}(q) \\
X_{p} & \mapsto \tau_{p}\left(Y_{p}\right) & X_{q} & \mapsto \tau_{q}\left(Y_{q}\right),
\end{aligned}
$$


where $p \in E_{\mathrm{SO}(p)}, q \in E_{\mathrm{SO}(q)}$, the vector fields $Y_{p}$ and $Y_{q}$ are the vertical components of $X_{p}$ and $X_{q}$ according to the decomposition in vertical and horizontal vector fields given by the connections, and $\tau_{p}$ and $\tau_{q}$ are the isomorphisms between the vertical subspaces and the Lie algebras. If $\xi \in \mathfrak{s o}(p)$,

$$
\tau_{p}(\xi)=\gamma^{\prime}(0)
$$

where $\gamma$ is the curve in $E_{\mathrm{SO}(p)}$ defined by

$$
\gamma(t)=p \cdot \exp (t \xi)
$$

The analogous definition for $\tau_{q}$. A gauge transformation $g=\left(g_{1}, g_{2}\right) \in \mathcal{G}\left(E_{\mathrm{SO}(p)}(\mathrm{SO}(p))\right) \times$ $\mathcal{G}\left(E_{\mathrm{SO}(q)}(\mathrm{SO}(q))\right)$ acts on the pair $\left(\omega_{1}, \omega_{2}\right)$ as follows,
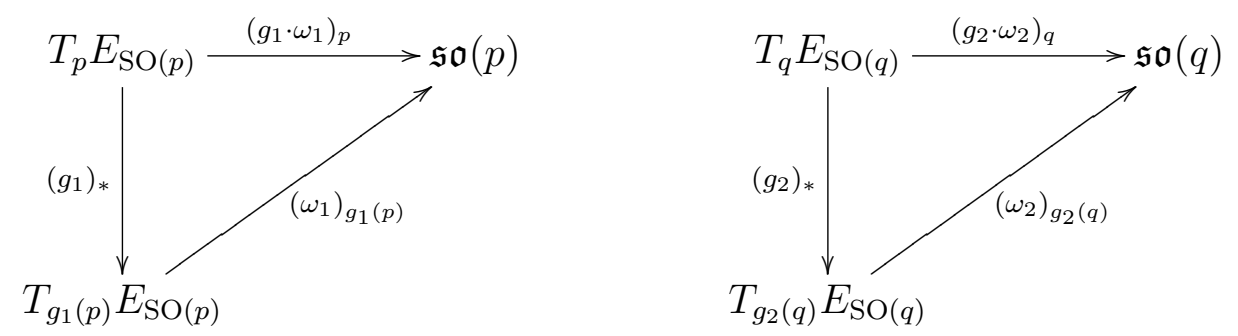

that is,

$$
\begin{aligned}
& \left(g_{1} \cdot \omega_{1}\right)_{p}\left(X_{p}\right)=\left(\omega_{1}\right)_{g_{1}(p)}\left(\left(g_{1}\right)_{*}\left(x_{p}\right)\right), \\
& \left(g_{2} \cdot \omega_{2}\right)_{q}\left(X_{q}\right)=\left(\omega_{2}\right)_{g_{2}(q)}\left(\left(g_{2}\right)_{*}\left(x_{q}\right)\right),
\end{aligned}
$$

and then

$$
g_{i} \cdot \omega_{i}=g_{i}^{*}\left(\omega_{i}\right)
$$

Let $\left(V, Q_{V}\right)$ and $\left(W, Q_{W}\right)$ be the vector bundles associated to the principal bundles $E_{\mathrm{SO}(p)}$ and $E_{\mathrm{SO}(q)}$, that is,

$$
\begin{aligned}
& V=E_{\mathrm{SO}(p)} \times_{\mathrm{SO}(p)} \mathbb{R}^{p}, \\
& W=E_{\mathrm{SO}(q)} \times_{\mathrm{SO}(q)} \mathbb{R}^{q},
\end{aligned}
$$

with $\operatorname{det} V=\operatorname{det} W=\mathbb{R}$ and $Q_{V}, Q_{W}$ are two non-degenerate symmetric quadratic forms on $V$ and $W$ respectively. A connection $A=\left(A_{1}, A_{2}\right)$ defines a pair of covariant derivatives

$$
d_{A_{1}}: \Omega^{p}(V) \rightarrow \Omega^{p+1}(V), \quad d_{A_{2}}: \Omega^{p}(W) \rightarrow \Omega^{p+1}(W)
$$

with

$$
d_{A_{i}}(\sigma \cdot \varphi)=\left(d_{A_{i}} \sigma\right) \wedge \varphi+\sigma \cdot d \varphi
$$


for all $\sigma \in \Omega^{0}(V)$ (resp. $\Omega^{0}(W)$ ) and $\varphi \in \Omega^{p}$, preserving the forms $Q_{V}$ and $Q_{W}$,

$$
\begin{gathered}
d\left(Q_{V}(\varphi, \psi)\right)=Q_{V}\left(d_{A_{1}} \varphi, \psi\right)+Q_{V}\left(\varphi, d_{A_{1}} \psi\right), \varphi, \psi \in \Omega^{p}(V) \\
d\left(Q_{W}(\varphi, \psi)\right)=Q_{W}\left(d_{A_{2}} \varphi, \psi\right)+Q_{W}\left(\varphi, d_{A_{2}} \psi\right), \varphi, \psi \in \Omega^{p}(W) .
\end{gathered}
$$

An element $\left(g_{1}, g_{2}\right) \in \mathcal{G}(V) \times \mathcal{G}(W)$ acts on $\left(d_{A_{1}}, d_{A_{2}}\right)$ by

$$
g_{i} \cdot d_{A_{i}}=g_{i} \circ d_{A_{i}} \circ g_{i}^{-1}=g_{i} d_{A_{i}} g_{i}^{-1}+d_{A_{i}} .
$$

Finally, if $g=\left(g_{1}, g_{2}\right)$ is an element of $\Omega^{0}\left(E_{\mathrm{SO}(p)}(\mathrm{SO}(p))\right) \times \Omega^{0}\left(E_{\mathrm{SO}(q)}(\mathrm{SO}(q))\right)$ and $x \in S$, we have $g_{x}=\left(\left(g_{1}\right)_{x},\left(g_{2}\right)_{x}\right) \in \mathrm{SO}(p) \times \mathrm{SO}(q)$ and $\psi_{x}$ is locally an element of $\mathfrak{m}$, then $g_{x}$ acts on $\psi_{x}$ through the isotropy representation

$$
\iota: \mathrm{SO}(p) \times \mathrm{SO}(q) \rightarrow \mathrm{GL}(\mathfrak{m})
$$

that is, if $\psi_{x}=\left(\begin{array}{cc} & X_{2} \\ X_{2}^{t} & \end{array}\right) \in \mathfrak{m}$,

$$
\begin{aligned}
g_{x} \cdot \psi_{x}=\iota\left(g_{x}\right)\left(\psi_{x}\right) & =\left(\begin{array}{ll}
\left(g_{1}\right)_{x} & \\
& \left(g_{2}\right)_{x}
\end{array}\right)\left(\begin{array}{c}
X_{2} \\
X_{2}^{t}
\end{array}\right)\left(\begin{array}{ll}
\left(g_{1}\right)_{x}^{-1} & \\
& \left(g_{2}\right)_{x}^{-1}
\end{array}\right) \\
& =\left(\begin{array}{cc}
\left(g_{1}\right)_{x} X_{2}\left(g_{2}\right)_{x}^{-1} \\
\left(g_{2}\right)_{x} X_{2}^{t}\left(g_{1}\right)_{x}^{-1}
\end{array}\right.
\end{aligned}
$$

We can write

$$
g \cdot \psi=\iota(g)(\psi)=g \psi g^{-1}
$$

We can express the moment map for the action of $\mathcal{G}(F)$ on the pairs $(A, \psi)$ as

$$
\Phi=d_{A}^{*} \psi
$$

where $d_{A}^{*}$ is the adjoint of $d_{A}$. The reduction of structure group from $\mathrm{SO}_{0}(p, q)$ to $\mathrm{SO}(p) \times$ $\mathrm{SO}(q)$ is said to be harmonic if this moment map vanishes, that is

$$
d_{A}^{*} \psi=0
$$

This condition of harmonicity for the reduction $h$ is equivalent to the condition for the map

$$
\tilde{h}: \tilde{S} \rightarrow \mathrm{SO}_{0}(p, q) / \mathrm{SO}(p) \times \mathrm{SO}(q),
$$

to be harmonic as a map of Riemannian manifolds because the 1-form $\psi$ can be identified with the differential of the map $\tilde{h}$ and $d_{A}$ with the pullback of the Levi-Civita connection 
on $\mathrm{SO}_{0}(p, q) / \mathrm{SO}(p) \times \mathrm{SO}(q)($ see [14]).

Let $\rho$ be a representation of $\pi_{1}$ in $\mathrm{SO}_{0}(p, q)$ and let $(E, D)$ be its associated flat principal bundle. Consider a harmonic reduction $F \subset E$. As we have seen in the previous section, the connection $D$ admits a splitting

$$
D=A+\psi
$$

where $A$ is a connection on $F$ and $\psi \in \Omega^{1}(F(\mathfrak{m}))$. Since $D$ is flat, it satisfies the following equation

$$
F_{D}=F_{A}+\frac{1}{2}[\psi, \psi]+d_{A}(\psi)=0
$$

where $F_{A}+\frac{1}{2}[\psi, \psi]$ takes values in $\mathfrak{h}$ and $d_{A}(\psi)$ in $\mathfrak{m}$. Then, the pair $(A, \psi)$ satisfies the equations

$$
\left\{\begin{aligned}
F_{A}+\frac{1}{2}[\psi, \psi] & =0 \\
d_{A}(\psi) & =0 \\
d_{A}^{*}(\psi) & =0
\end{aligned}\right.
$$

where $[\psi, \psi]=\psi \psi+\psi \psi$, which are called harmonic equations. First and second equalities correspond to $F_{D}=0$ and the last equation is the condition of harmonicity for the reduction to $\mathrm{SO}(p) \times \mathrm{SO}(q)$.

A theorem of Corlette [13] applied to $\mathrm{SO}_{0}(p, q)$ says the following:

Theorem 4.4. If $\rho$ is a representation of $\pi_{1}$ in $\mathrm{SO}_{0}(p, q)$ and $(E, D)$ is the associated flat principal bundle, the representation $\rho$ is reductive if and only if $E$ admits a harmonic reduction to $\mathrm{SO}(p) \times \mathrm{SO}(q)$.

We have seen in Section 4.1 that there is a correspondence

$$
\mathcal{R}_{c}\left(\mathrm{SO}_{0}(p, q)\right) \cong \mathcal{F}_{c}\left(\mathrm{SO}_{0}(p, q)\right)
$$

Now, Theorem 4.4 gives a bijection between the space $\mathcal{F}_{c}\left(\mathrm{SO}_{0}(p, q)\right)$ and the moduli space

$$
\begin{aligned}
\mathcal{M}_{c}^{\text {Har }}\left(\mathrm{SO}_{0}(p, q)\right)= & \{(A, \psi) \mid A \text { connection on a principal } \mathrm{SO}(p) \times \mathrm{SO}(q) \text {-bundle } F \\
& \left.\psi \in \Omega^{1}(F(\mathfrak{m})), \text { satisfying }(1) \text { and with } c(F)=c\right\} / \mathcal{G}(F) .
\end{aligned}
$$

Then, we have

$$
\mathcal{R}_{c}\left(\mathrm{SO}_{0}(p, q)\right) \cong \mathcal{F}_{c}\left(\mathrm{SO}_{0}(p, q)\right) \cong \mathcal{M}_{c}^{H a r}\left(\mathrm{SO}_{0}(p, q)\right)
$$




\subsection{Hitchin's equations and Higgs bundles}

We describe now the moduli space $\mathcal{M}_{c}\left(\mathrm{SO}_{0}(p, q)\right)$ in terms of Dolbeault operators.

If $\mathbf{E}$ is a smooth principal $\mathrm{SO}(p, \mathbb{C}) \times \mathrm{SO}(q, \mathbb{C})$-bundle, a Dolbeault operator is a $\mathbb{C}$-linear map

$$
\bar{\partial}_{E}: \Omega^{0}(X, \mathbf{E}) \rightarrow \Omega^{0,1}(X, \mathbf{E}),
$$

which satisfy

$$
\bar{\partial}_{E}(f \xi)=\bar{\partial}_{E} f \xi+f \bar{\partial}_{E} \xi
$$

where $f \in \Omega^{0}(X)$ and $\xi \in \Omega^{0}(X, \mathbf{E})$. This can be extended to a $\mathbb{C}$-linear map

$$
\bar{\partial}_{E}: \Omega^{0,1}(X, \mathbf{E}) \rightarrow \Omega^{0,2}(X, \mathbf{E})
$$

Since $X$ is a Riemann surface, we have $\Omega^{0,2}(X, \mathbf{E})=0$ and hence all Dolbeault operators in $\mathbf{E}$ satisfy $\bar{\partial}_{E}^{2}=0$. This means that each Dolbeault operator $\bar{\partial}_{E}$ on $\mathbf{E}$ defines a holomorphic structure on $\mathbf{E}$ (see [55], Appendix). Denote $E$ the corresponding holomorphic principal $\mathrm{SO}(p, \mathbb{C}) \times \mathrm{SO}(q, \mathbb{C})$-bundle.

We thus have a correspondence between the moduli space of polystable $\mathrm{SO}_{0}(p, q)$ Higgs bundles and the moduli space of polystable pairs $\left(\bar{\partial}_{E}, \varphi\right)$, where $\bar{\partial}_{E}$ is a Dolbeault operator on a principal $\mathrm{SO}(p, \mathbb{C}) \times \mathrm{SO}(q, \mathbb{C})$-bundle $\mathbf{E}$ and $\varphi \in \Omega^{1,0}\left(\mathbf{E}\left(\mathfrak{m}^{\mathbb{C}}\right)\right)$, satisfying the condition $\bar{\partial}_{E}(\varphi)=0$, which is equivalent to $\varphi \in H^{0}\left(E\left(\mathfrak{m}^{\mathbb{C}}\right) \otimes K\right)$, modulo de gauge $\operatorname{group} \mathcal{G}(\mathbf{E})$,

$$
\begin{aligned}
\mathcal{M}_{c}\left(\mathrm{SO}_{0}(p, q)\right) \cong & \left\{\text { Polystable }\left(\bar{\partial}_{E}, \varphi\right) \mid \bar{\partial}_{E} \text { on a smooth principal } \mathrm{SO}(p, \mathbb{C}) \times \operatorname{SO}(q, \mathbb{C})-\right. \\
& \text { bundle } \left.\mathbf{E}, \varphi \in \Omega^{1,0}\left(\mathbf{E}\left(\mathfrak{m}^{\mathbb{C}}\right)\right), \bar{\partial}_{E}(\varphi)=0 \text { and } c(\mathbf{E})=c\right\} / \mathcal{G}(\mathbf{E}) .
\end{aligned}
$$

The gauge group $\mathcal{G}(\mathbf{E})$ acts on the operators $\bar{\partial}_{E}$ by the rule

$$
g \cdot \bar{\partial}_{E}=g \bar{\partial}_{E} g^{-1}
$$

If $g=\left(g_{1}, g_{2}\right) \in \mathcal{G}(\mathbf{E}) \cong \Omega^{0}\left(E_{\mathrm{SO}(p, \mathbb{C})}(\mathrm{SO}(p, \mathbb{C}))\right) \times \Omega^{0}\left(E_{\mathrm{SO}(q, \mathbb{C})}(\mathrm{SO}(q, \mathbb{C}))\right)$, where

$$
\mathbf{E}=E_{\mathrm{SO}(p, \mathbb{C})} \times E_{\mathrm{SO}(q, \mathbb{C})}
$$

and $x \in X$, then $g_{x}=\left(\left(g_{1}\right)_{x},\left(g_{2}\right)_{x}\right)$ is in $\operatorname{SO}(p, \mathbb{C}) \times \operatorname{SO}(q, \mathbb{C})$ and $\varphi_{x}$ is locally an element of $\mathfrak{m}^{\mathbb{C}}$. Then $g_{x}$ acts on $\varphi_{x}$ through the complexified isotropy representation

$$
\iota: \mathrm{SO}(p, \mathbb{C}) \times \mathrm{SO}(q, \mathbb{C}) \rightarrow \mathrm{GL}\left(\mathfrak{m}^{\mathbb{C}}\right)
$$


that is, if $\varphi_{x}=\left(\begin{array}{cc}X_{2} \\ -X_{2}^{t} & \end{array}\right) \in \mathfrak{m}^{\mathbb{C}}$

$$
\begin{aligned}
g_{x} \cdot \varphi_{x}=\iota\left(g_{x}\right)\left(\varphi_{x}\right) & =\left(\begin{array}{ll}
\left(g_{1}\right)_{x} & \\
& \left(g_{2}\right)_{x}
\end{array}\right)\left(\begin{array}{cc}
X_{2} \\
-X_{2}^{t}
\end{array}\right)\left(\begin{array}{ll}
\left(g_{1}\right)_{x}^{-1} & \\
& \left(g_{2}\right)_{x}^{-1}
\end{array}\right) \\
& =\left(\begin{array}{cc} 
& \left(g_{1}\right)_{x} X_{2}\left(g_{2}\right)_{x}^{-1} \\
-\left(g_{2}\right)_{x} X_{2}^{t}\left(g_{1}\right)_{x}^{-1} &
\end{array}\right) .
\end{aligned}
$$

We can write

$$
g \cdot \varphi=\iota(g)(\varphi)=g \varphi g^{-1}
$$

Let $\left(V, Q_{V}\right)$ and $\left(W, Q_{W}\right)$ be the vector bundles associated to $E$. The Higgs field is of the form

$$
\eta: W \rightarrow V \otimes K
$$

In terms of $V$ and $W$, a gauge transformation $g$ is a pair $\left(g_{1}, g_{2}\right)$ of automorphisms of $V$ and $W$ respectively. We have the commutative diagram

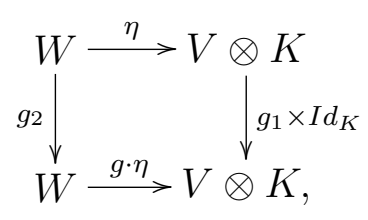

and the action of $g=\left(g_{1}, g_{2}\right)$ on $\eta$ is given by

$$
g \cdot \eta=g_{1} \circ \eta \circ g_{2}^{-1}
$$

If we consider the isotropy representation

$$
\iota: \mathrm{SO}(V) \times \mathrm{SO}(W) \rightarrow E\left(\mathfrak{m}^{\mathbb{C}}\right) \otimes K \cong \operatorname{Hom}(W, V) \otimes K
$$

we have

$$
g \cdot \eta=\iota(g)(\eta)=\left(\begin{array}{cc}
g_{1} \circ \eta \circ g_{2}^{-1} \\
-g_{2} \circ \eta^{\top} \circ g_{1}^{-1}
\end{array}\right) \cong g_{1} \circ \eta \circ g_{2}^{-1}
$$

If $\mathbf{E}$ is a smooth principal $\mathrm{SO}(p, \mathbb{C}) \times \mathrm{SO}(q, \mathbb{C})$-bundle, there is a correspondence between Dolbeault operators on $\mathbf{E}$ and connections on the reduction $F$ of $\mathbf{E}$ to $\mathrm{SO}(p) \times$ $\mathrm{SO}(q)$, which is given by

$$
\begin{aligned}
d_{A} & \rightarrow \bar{\partial}_{A}, \\
\bar{\partial}+d_{A}^{1,0} & \leftarrow \bar{\partial},
\end{aligned}
$$

where $d_{A}^{1,0}$ is determined by the reduction. 
Consider a pair $(A, \varphi)$, where $A$ is a connection on the smooth principal $\mathrm{SO}(p) \times \mathrm{SO}(q)$ bundle $F$ and $\varphi \in \Omega^{1,0}\left(F\left(\mathfrak{m}^{\mathbb{C}}\right)\right)$. Let $\tau$ denote the conjugation on $\mathfrak{s o}(p+q, \mathbb{C})$ that defines the compact real form

$$
\begin{aligned}
\mathfrak{s o}(p+q) & =\{X \in \mathfrak{s o}(p+q, \mathbb{C}) \mid X=\bar{X}\}= \\
& =\left\{X \in \mathfrak{s l}(p+q, \mathbb{R}) \mid X+X^{t}=0\right\}
\end{aligned}
$$

that is, $\tau(X)=\bar{X}$. Since $\tau$ and the Cartan involution commute, we have $\tau\left(\mathfrak{m}^{\mathbb{C}}\right) \subseteq \mathfrak{m}^{\mathbb{C}}$ and then $\tau$ preserves the Cartan decomposition $\mathfrak{s o}(p+q, \mathbb{C})=\mathfrak{s o}(p, \mathbb{C}) \times \mathfrak{s o}(q, \mathbb{C}) \oplus \mathfrak{m}^{\mathbb{C}}$. This conjugation induces a $\tau$ in $E\left(\mathfrak{m}^{\mathbb{C}}\right)$ and then it makes sense to apply $\tau$ to the section $\varphi$.

The Hitchin's equations corresponding to the pair $(A, \varphi)$, where $A$ is a connection on a smooth principal $\mathrm{SO}(p) \times \mathrm{SO}(q)$-bundle $F$ and $\varphi \in \Omega^{1,0}\left(F\left(\mathfrak{m}^{\mathbb{C}}\right)\right)$, are

$$
\left\{\begin{aligned}
F_{A}-[\varphi, \tau(\varphi)] & =0 \\
\bar{\partial}_{A}(\varphi) & =0
\end{aligned}\right.
$$

Since $\tau(\eta)=\bar{\eta}$, we can compute

$$
\begin{aligned}
{[\varphi, \tau(\varphi)] } & =\left(\begin{array}{rr} 
& \eta \\
-\eta^{\top} &
\end{array}\right)\left(\begin{array}{rr} 
& \bar{\eta} \\
-\bar{\eta}^{\top} &
\end{array}\right)+\left(\begin{array}{rr}
\bar{\eta} \\
-\bar{\eta}^{\top}
\end{array}\right)\left(\begin{array}{c}
\eta \\
-\eta^{\top}
\end{array}\right)= \\
& =\left(\begin{array}{c}
-\eta \bar{\eta}^{\top}-\bar{\eta} \eta^{\top} \\
-\eta^{\top} \bar{\eta}-\bar{\eta}^{\top} \eta
\end{array}\right),
\end{aligned}
$$

and if the connection is $A=\left(A_{1}, A_{2}\right)$, we get from $F_{A}-[\varphi, \tau(\varphi)]=0$ the pair of equations

$$
\begin{aligned}
& F_{A_{1}}+\eta \bar{\eta}^{\top}+\bar{\eta} \eta^{\top}=F_{A_{1}}+2 \operatorname{Re}\left(\eta \bar{\eta}^{\top}\right)=0, \\
& F_{A_{2}}+\eta^{\top} \bar{\eta}+\bar{\eta}^{\top} \eta=F_{A_{2}}+2 \operatorname{Re}\left(\eta^{\top} \bar{\eta}\right)=0 .
\end{aligned}
$$

If we fix the pair $(A, \varphi)$, a solution to the Hitchin's equations is a reduction from $\mathrm{SO}(p, \mathbb{C}) \times \mathrm{SO}(q, \mathbb{C})$ to $\mathrm{SO}(p) \times \mathrm{SO}(q)$ and the connection $A$ is the unique connection on $F$ compatible with the holomorphic structure of $E$, that is, the part $(0,1)$ of the covariant derivative $d_{A}, \bar{\partial}_{A}$, is the Dolbeault operator that defines the holomorphic structure of $E$. But, if we fix the reduction and $F$ is a principal $\mathrm{SO}(p) \times \mathrm{SO}(q)$-bundle, then a solution of the Hitchin's equations is a pair $(A, \varphi)$, where $A$ is a connection on $F$ and $\varphi \in \Omega^{1,0}\left(F\left(\mathfrak{m}^{\mathbb{C}}\right)\right)$, satisfying

$$
\left\{\begin{aligned}
F_{A}-[\varphi, \tau(\varphi)] & =0 \\
\bar{\partial}_{A}(\varphi) & =0
\end{aligned}\right.
$$


The moduli space of solutions to the Hitchin's equations is

$$
\begin{aligned}
\mathcal{M}_{c}^{H i t}\left(\mathrm{SO}_{0}(p, q)\right)= & \{(A, \varphi) \mid A \text { connection on a smooth principal } \mathrm{SO}(p) \times \mathrm{SO}(q) \text {-bundle } \\
& \left.F, \varphi \in \Omega^{1,0}\left(F\left(\mathfrak{m}^{\mathbb{C}}\right)\right) \text { satisfying }(2) \text { and with } c(F)=c\right\} / \mathcal{G}(F) .
\end{aligned}
$$

We have described in Section 4.2 the action of $\mathcal{G}(F)$ on the connection $A$ and the action of $\mathcal{G}(F)$ on $\varphi \in \Omega^{1,0}\left(F\left(\mathfrak{m}^{\mathbb{C}}\right)\right)$ is analogous to the action on $\varphi \in \Omega^{1,0}\left(\mathbf{E}\left(\mathfrak{m}^{\mathbb{C}}\right)\right)$ explained in this section. If $g=\left(g_{1}, g_{2}\right) \in \mathcal{G}(F)=\Omega^{0}\left(E_{\mathrm{SO}(p)}(\mathrm{SO}(p))\right) \times \Omega^{0}\left(E_{\mathrm{SO}(q)}(\mathrm{SO}(q))\right)$ and $x \in X$, then $g_{x}=\left(\left(g_{1}\right)_{x},\left(g_{2}\right)_{x}\right)$ is in $\mathrm{SO}(p) \times \mathrm{SO}(q)$ and $\varphi_{x}$ is in $\mathfrak{m}^{\mathbb{C}}$. Then $g_{x}$ acts on $\varphi_{x}$ through the isotropy representation

$$
\iota: \mathrm{SO}(p) \times \mathrm{SO}(q) \subset \mathrm{SO}(p, \mathbb{C}) \times \mathrm{SO}(q, \mathbb{C}) \rightarrow \mathrm{GL}\left(\mathfrak{m}^{\mathbb{C}}\right) .
$$

that is, if $\varphi_{x}=\left(\begin{array}{cc}0 & X_{2} \\ -X_{2}^{t} & 0\end{array}\right) \in \mathfrak{m}^{\mathbb{C}}$,

$$
\begin{aligned}
g_{x} \cdot \varphi_{x}=\iota\left(g_{x}\right)\left(\varphi_{x}\right) & =\left(\begin{array}{cc}
\left(g_{1}\right)_{x} & 0 \\
0 & \left(g_{2}\right)_{x}
\end{array}\right)\left(\begin{array}{cc}
0 & X_{2} \\
-X_{2}^{t} & 0
\end{array}\right)\left(\begin{array}{cc}
\left(g_{1}\right)_{x}^{-1} & 0 \\
0 & \left(g_{2}\right)_{x}^{-1}
\end{array}\right) \\
& =\left(\begin{array}{cc}
0 & \left(g_{1}\right)_{x} X_{2}\left(g_{2}\right)_{x}^{-1} \\
-\left(g_{2}\right)_{x} X_{2}^{t}\left(g_{1}\right)_{x}^{-1} & 0
\end{array}\right) .
\end{aligned}
$$

We can write

$$
g \cdot \varphi=\iota(g)(\varphi)=g \varphi g^{-1}
$$

Theorems of Bradlow, García-Prada and Mundet i Riera [10] for an arbitrary reductive real Lie group when the Higgs bundle is stable and García-Prada, Gothen and Mundet i Riera [19, Theorem 2.19] for the general polystable case, give the following.

Theorem 4.5. An $\mathrm{SO}_{0}(p, q)$-Higgs bundle $(E, \varphi)$ is polystable if and only if the principal $\mathrm{SO}(p, \mathbb{C}) \times \mathrm{SO}(q, \mathbb{C})$-bundle $E$ admits a reduction to $\mathrm{SO}(p) \times \mathrm{SO}(q)$ such that

$$
F_{A}-[\varphi, \tau(\varphi)]=0
$$

where $A$ is the connection on the principal $\mathrm{SO}(p) \times \mathrm{SO}(q)$-bundle $F$ corresponding to the operator $\bar{\partial}_{E}$ that defines the holomorphic structure of $E$.

This result gives a correspondence between the moduli space of $\mathrm{SO}_{0}(p, q)$-Higgs bundles and the moduli space of solutions of the Hitchin's equations,

$$
\mathcal{M}_{c}\left(\mathrm{SO}_{0}(p, q)\right) \cong \mathcal{M}_{c}^{H i t}\left(\mathrm{SO}_{0}(p, q)\right)
$$




\subsection{The correspondence}

The proof of the following result is given by Theorem 4.4 and Theorem 4.5 .

Theorem 4.6. There is a homeomorphism

$$
\mathcal{R}_{c}\left(\mathrm{SO}_{0}(p, q)\right) \cong \mathcal{M}_{c}\left(\mathrm{SO}_{0}(p, q)\right)
$$

Theorem 4.4 gives a homeomorphism between the moduli space of representations and the moduli space of solutions to the harmonic equations,

$$
\mathcal{R}_{c}\left(\mathrm{SO}_{0}(p, q)\right) \cong \mathcal{M}_{c}^{\text {Har }}\left(\mathrm{SO}_{0}(p, q)\right)
$$

and Theorem 4.5 gives a homeomorphism between the moduli space of Higgs bundles and the moduli space of solutions to Hitchin's equations,

$$
\mathcal{M}_{c}\left(\mathrm{SO}_{0}(p, q)\right) \cong \mathcal{M}_{c}^{H i t}\left(\mathrm{SO}_{0}(p, q)\right)
$$

To prove the result of Theorem 4.6 we need to establish a bijection between the spaces of solutions $\mathcal{M}_{c}^{\text {Har }}\left(\mathrm{SO}_{0}(p, q)\right)$ and $\mathcal{M}_{c}^{\text {Hit }}\left(\mathrm{SO}_{0}(p, q)\right)$.

If $(A, \psi)$ represents a class in $\mathcal{M}_{c}^{\text {Har }}\left(\mathrm{SO}_{0}(p, q)\right)$ and we extend the structure group from $\mathrm{SO}(p) \times \mathrm{SO}(q)$ to $\mathrm{SO}(p, \mathbb{C}) \times \mathrm{SO}(q, \mathbb{C})$, the extended connection and curvature are of the form

$$
\begin{gathered}
d_{A}=d_{A}^{1,0}+d_{A}^{0,1}, \\
F_{A}=F_{A}^{2,0}+F_{A}^{1,1}+F_{A}^{0,2}=F_{A}^{1,1},
\end{gathered}
$$

since $X$ is a Riemann surface and then $F_{A}^{2,0}=F_{A}^{0,2}=0$. We denote $\bar{\partial}_{A}=d_{A}^{0,1}$. Analogously,

$$
\psi=\psi^{1,0}+\psi^{0,1}=\varphi-\tau(\varphi)
$$

with $\varphi \in \Omega^{1,0}\left(F\left(\mathfrak{m}^{\mathbb{C}}\right)\right)$ and $\tau$ being the conjugation in $\mathfrak{s o}(p+q, \mathbb{C})$ that defines the compact real form $\mathfrak{s o}(p+q)$ as we have seen in Section 4.3. Rewriting the harmonic equations in these terms, we obtain the Hitchin's equations

$$
\left\{\begin{aligned}
F_{A}-[\varphi, \tau(\varphi)] & =0 \\
\bar{\partial}_{A}(\varphi) & =0 \\
\left(F_{A}^{0,2}=[\varphi, \varphi]\right. & =0)
\end{aligned}\right.
$$

That is, the map

$$
(A, \varphi) \mapsto(A, \psi=\varphi-\tau(\varphi))
$$


defines a homeomorphism

$$
\mathcal{M}_{c}^{H i t}\left(\mathrm{SO}_{0}(p, q)\right) \cong \mathcal{M}_{c}^{H a r}\left(\mathrm{SO}_{0}(p, q)\right)
$$

and we obtain the result of Theorem 4.6,

All the correspondences used in this proof are outlined in the following diagram:
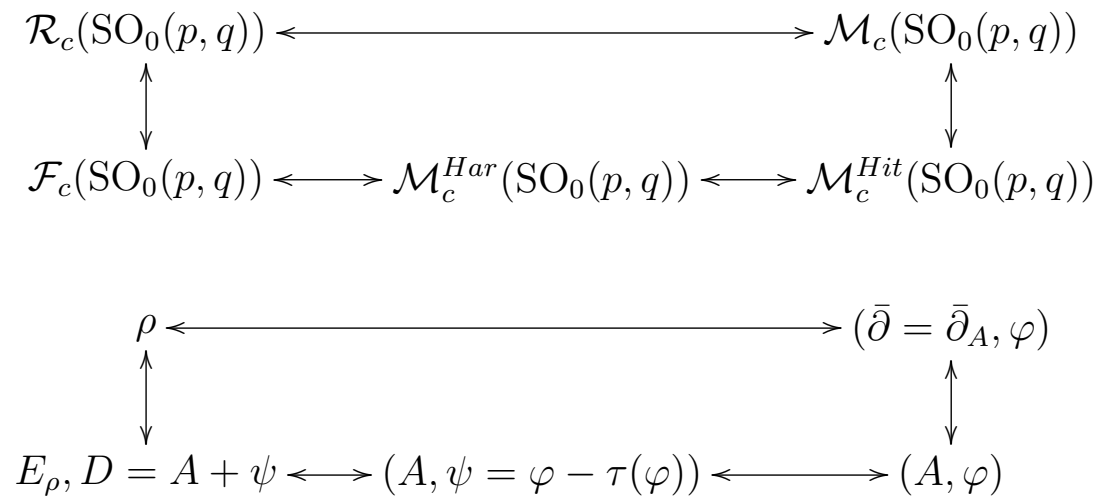

Using this homeomorphism we can obtain information about the topological properties of $\mathcal{R}_{c}\left(\mathrm{SO}_{0}(p, q)\right)$ studying the topological properties of $\mathcal{M}_{c}\left(\mathrm{SO}_{0}(p, q)\right)$. For example, counting the number of connected components of each $\mathcal{R}_{c}\left(\mathrm{SO}_{0}(p, q)\right)$ is the same as counting the number of the connected components of $\mathcal{M}_{c}\left(\mathrm{SO}_{0}(p, q)\right)$. 



\section{Low rank isomorphisms}

In this chapter, using the isomorphisms between Spin Lie groups and other classical semisimple Lie groups described in [28, Theorem 14.1], we study the relation between the moduli space of polystable $\mathrm{SO}(n, \mathbb{C})$-Higgs bundles with $n=3,4,5$ and 6 and the moduli space of polystable $G$-Higgs bundles, where $G=\operatorname{SL}(2, \mathbb{C}), \operatorname{SL}(2, \mathbb{C}) \times \operatorname{SL}(2, \mathbb{C})$, $\mathrm{Sp}(4, \mathbb{C})$ and $\mathrm{SL}(4, \mathbb{C})$ respectively, and the relations between the real forms $\mathrm{SO}_{0}(p, q)$, with $p+q=n$, and the corresponding real forms of the Lie group $G$.

Using the classification of semisimple complex Lie algebras given by the Dynkin diagrams, we observe the isomorphisms:

$$
\begin{aligned}
\mathfrak{s o}(3, \mathbb{C}) & \cong \mathfrak{s l}(2, \mathbb{C}) \cong \mathfrak{s p}(2, \mathbb{C}) \\
\mathfrak{s o}(4, \mathbb{C}) & \cong \mathfrak{s l}(2, \mathbb{C}) \times \mathfrak{s l}(2, \mathbb{C}) \\
\mathfrak{s o}(5, \mathbb{C}) & \cong \mathfrak{s p}(4, \mathbb{C}) \\
\mathfrak{s o}(6, \mathbb{C}) & \cong \mathfrak{s l}(4, \mathbb{C})
\end{aligned}
$$

Each isomorphism induces isomorphisms between the corresponding real forms. To deal with the real forms, we use the following result (see [29]).

Theorem 5.1. Suppose that $\mathfrak{g}_{1}$ and $\mathfrak{g}_{2}$ are real forms of the same simple complex Lie algebra $\mathfrak{g}$, and let $\mathfrak{g}_{1}=\mathfrak{h}_{1}+\mathfrak{m}_{1}$ and $\mathfrak{g}_{2}=\mathfrak{h}_{2}+\mathfrak{m}_{2}$ be any Cartan decompositions. If $\mathfrak{h}_{1}$ and $\mathfrak{h}_{2}$ are isomorphic, $\mathfrak{g}_{1}$ and $\mathfrak{g}_{2}$ are isomorphic.

Hence, the isomorphisms between the compact real forms:

$$
\begin{aligned}
& \mathfrak{s o}(3) \cong \mathfrak{s u}(2) \cong \mathfrak{s p}(2), \\
& \mathfrak{s o}(4) \cong \mathfrak{s u}(2) \times \mathfrak{s u}(2), \\
& \mathfrak{s o}(5) \cong \mathfrak{s p}(4), \\
& \mathfrak{s o}(6) \cong \mathfrak{s u}(4),
\end{aligned}
$$

induce isomorphisms between the non-compact real forms:

$$
\begin{array}{ll}
\mathfrak{s o}(2,1) \cong \mathfrak{s l}(2, \mathbb{R}), & \mathfrak{s o}(3,3) \cong \mathfrak{s l}(4, \mathbb{R}), \\
\mathfrak{s o}(2,2) \cong \mathfrak{s l}(2, \mathbb{R}) \times \mathfrak{s l}(2, \mathbb{R}), & \mathfrak{s o}(2,4) \cong \mathfrak{s u}(2,2), \\
\mathfrak{s o}(1,3) \cong \mathfrak{s l}(2, \mathbb{C}), & \\
& \\
\mathfrak{s o}(2,3) \cong \mathfrak{s o}(2,6) \cong \mathfrak{s u}^{*}(4), \\
\mathfrak{s o}(1,4) \cong \mathfrak{s p}(2,2) . &
\end{array}
$$


This gives local isomorphisms between the Lie groups $\mathrm{SO}_{0}(p, q)$ with $p+q=3,4,5,6$ and other semisimple classical Lie groups, and allow us to establish an arrow between the corresponding moduli spaces. Since the problem of counting the number of connected components of the moduli space of $G$-Higgs bundles is solved for several semisimple classical Lie groups, we will use these local isomorphisms to study the same problem for the moduli space $\mathcal{M}\left(\mathrm{SO}_{0}(p, q)\right)$ in these small rank cases.

\subsection{Isomorphisms of $\mathrm{SO}(3, \mathbb{C})$ and its real forms}

The Lie group $\mathrm{SO}(3, \mathbb{C})$ is locally isomorphic to the special linear group $\mathrm{SL}(2, \mathbb{C})$ and to the symplectic group $\operatorname{Sp}(2, \mathbb{C})$, but the Lie group with Lie algebra $\mathfrak{s o}(3, \mathbb{C})$ which is isomorphic to $\operatorname{SL}(2, \mathbb{C})$ and $\operatorname{Sp}(2, \mathbb{C})$ is the spin group $\operatorname{Spin}(3, \mathbb{C})$ (see [28, Theorem 14.1]). The same happens in the case of the real forms, we have isomorphisms

$$
\operatorname{Spin}(3) \cong \mathrm{SU}(2) \cong \operatorname{Sp}(2) \quad \text { and } \quad \operatorname{Spin}_{0}(2,1) \cong \mathrm{SL}(2, \mathbb{R})
$$

Spin groups are double covers of the special orthogonal groups, and then we have the short exact sequences

$$
\begin{aligned}
& 1 \rightarrow \mathbb{Z}_{2} \rightarrow \operatorname{Spin}(3) \cong \mathrm{SU}(2) \rightarrow \mathbb{Z}_{2} \rightarrow \mathrm{SO}(3) \rightarrow \operatorname{Spin}(3, \mathbb{C}) \cong \mathrm{SL}(2, \mathbb{C}) \rightarrow \mathrm{SO}(3, \mathbb{C}) \rightarrow 1 \\
& 1 \rightarrow \mathbb{Z}_{2} \rightarrow \mathbb{Z}_{2} \rightarrow \operatorname{Spin}_{0}(2,1) \cong \mathrm{SL}(2, \mathbb{R}) \rightarrow \mathrm{SO}_{0}(2,1) \rightarrow 1 \\
& 1 \rightarrow \mathbb{Z}_{2} \rightarrow
\end{aligned}
$$

Since we are interested in the moduli space of $\mathrm{SO}_{0}(p, q)$-Higgs bundles and not in spin Higgs bundles, we will use these sequences to establish arrows from the moduli space of $\mathrm{SU}(2), \mathrm{SL}(2, \mathbb{C})$ and $\mathrm{SL}(2, \mathbb{R})$-Higgs bundles to the moduli space of $\mathrm{SO}(3), \mathrm{SO}(3, \mathbb{C})$ and $\mathrm{SO}_{0}(2,1)$-Higgs bundles respectively.

We start with the compact real form $\mathrm{SO}(3)$. A SU(2)-Higgs bundle is a principal $\mathrm{SL}(2, \mathbb{C})$-bundle, or equivalently, a holomorphic vector bundle $E$ of rank 2 with trivial determinant. Let $F=S^{2} E$. This vector bundle has rank 3 and a non-degenerate symmetric quadratic form

$$
Q_{F}: S^{2} E \times S^{2} E \rightarrow \Lambda^{2} E \otimes \Lambda^{2} E=\mathcal{O}
$$

defined by

$$
Q_{F}\left(x \otimes y, x^{\prime} \otimes y^{\prime}\right)=\left(x \wedge x^{\prime}\right) \otimes\left(y \wedge y^{\prime}\right)+\left(x \wedge y^{\prime}\right) \otimes\left(y \wedge x^{\prime}\right)
$$

Hence, $\left(F=S^{2} E, Q_{F}\right)$ is a principal $\mathrm{SO}(3, \mathbb{C})$-bundle and we have the map

$$
\begin{aligned}
\mathcal{M}(\mathrm{SU}(2)) & \rightarrow \mathcal{M}(\mathrm{SO}(3)) \\
E & \mapsto\left(F=S^{2} E, Q_{F}\right) .
\end{aligned}
$$


A principal $\mathrm{SO}(3, \mathbb{C})$-bundle $F$ lifts to a principal $\mathrm{SL}(2, \mathbb{C})$-bundle if and only if the second Stiefel-Whitney class $\omega_{2}\left(F_{\mathrm{SO}(3)}\right)$ of the underlying orthogonal real bundle $F_{\mathrm{SO}(3)}$ is zero.

Consider now the sequence for the complex groups

$$
1 \rightarrow \mathbb{Z}_{2} \rightarrow \mathrm{SL}(2, \mathbb{C}) \rightarrow \mathrm{SO}(3, \mathbb{C}) \rightarrow 1
$$

An $\operatorname{SL}(2, \mathbb{C})$-Higgs bundle is a pair $(E, \phi)$, where $E$ is a holomorphic vector bundle of rank 2 and trivial determinant, and $\phi \in H^{0}\left(\operatorname{End}_{0}(E) \otimes K\right)$. On the other hand, as we have seen in Chapter 2, an $\mathrm{SO}(3, \mathbb{C})$-Higgs bundle is a triple $\left(F, Q_{F}, \varphi\right)$ consisting of a principal $\mathrm{SO}(3, \mathbb{C})$-bundle $\left(F, Q_{F}\right)$ and a holomorphic section $\varphi \in H^{0}(\mathfrak{s o}(F) \otimes K)$.

With the same construction as in the compact case, we obtain the principal $\mathrm{SO}(3, \mathbb{C})$ bundle $\left(F=S^{2} E, Q_{F}\right)$. Let $\left\{e_{1}, e_{2}\right\}$ be a basis of $E$, then $\left\{e_{1}^{2}, e_{1} e_{2}, e_{2}^{2}\right\}$ is a basis of $F$. If $\phi=\left(\begin{array}{cc}a & b \\ c & -a\end{array}\right)$ in this basis, we obtain the matrix of $\varphi$ in the corresponding basis of $F$ computing

$$
\varphi\left(e_{i} \otimes e_{j}\right)=\phi\left(e_{i}\right) \otimes e_{j}+e_{i} \otimes \phi\left(e_{j}\right)
$$

for $i, j=1,2$. We can also get the matrix of $Q_{F}$ in this basis computing $Q_{F}\left(e_{i} \otimes e_{j}, e_{k} \otimes e_{l}\right)$ with $i, j, k, l=1,2$. The resulting matrices are

$$
\varphi=\left(\begin{array}{ccc}
2 a & b & 0 \\
2 c & 0 & 2 b \\
0 & c & -2 a
\end{array}\right) \text { and } Q_{F}=\left(\begin{array}{ccc}
0 & 0 & 2 \\
0 & -1 & 0 \\
2 & 0 & 0
\end{array}\right)
$$

(Using $e_{1} \wedge e_{2}=1 \in \Lambda^{2} E \cong \mathcal{O}$ ). Since $\varphi^{t} Q_{F}+Q_{F} \varphi=0$, the Higgs field $\varphi$ is skewsymmetric and we can finally establish the map

$$
\begin{aligned}
\mathcal{M}(\mathrm{SL}(2, \mathbb{C})) & \rightarrow \mathcal{M}(\mathrm{SO}(3, \mathbb{C})) \\
\left(E, \phi=\left(\begin{array}{cc}
a & b \\
c & -a
\end{array}\right)\right) & \mapsto\left(F=S^{2} E, Q_{F}, \varphi=\left(\begin{array}{ccc}
2 a & b & 0 \\
2 c & 0 & 2 b \\
0 & c & -2 a
\end{array}\right)\right) .
\end{aligned}
$$

As in the compact case, an $\mathrm{SO}(3, \mathbb{C})$-Higgs bundle $\left(F, Q_{F}, \varphi\right)$ lifts to an $\mathrm{SL}(2, \mathbb{C})$-Higgs bundle if and only if the second Stiefel-Whitney class $\omega_{2}\left(F_{\mathrm{SO}(3)}\right)$ of the underlying orthogonal real bundle $F_{\mathrm{SO}(3)}$ is zero.

For the split real form $\mathrm{SO}_{0}(2,1)$ we have the following sequence

$$
1 \rightarrow \mathbb{Z}_{2} \rightarrow \mathrm{SL}(2, \mathbb{R}) \rightarrow \mathrm{SO}_{0}(2,1) \rightarrow 1
$$


An $\operatorname{SL}(2, \mathbb{R})$-Higgs bundle is a triple $\left(E=L \oplus L^{-1}, Q=\left(\begin{array}{ll}0 & 1 \\ 1 & 0\end{array}\right), \phi\right)$ where $L$ is a holomorphic line bundle, $Q \in H^{0}\left(S^{2} E^{*}\right)$ is the dual pairing and $\phi$ is a symmetric trace-free endomorphism of $E$, that is, $\phi=\left(\begin{array}{ll}0 & b \\ c & 0\end{array}\right)$ with $b \in H^{0}\left(L^{2} \otimes K\right)$ and $c \in H^{0}\left(L^{-2} \otimes K\right)$.

If we consider

$$
F=S^{2} E=S^{2}\left(L \oplus L^{-1}\right)=L^{2} \oplus \mathcal{O} \oplus L^{-2}=V \oplus W
$$

and take $V=L^{2} \oplus L^{-2}$ and $W=\mathcal{O}$, since $Q_{F}$ restricts to $V$ and $W$, we obtain two orthogonal bundles $\left(V, Q_{V}\right)$ and $\left(W, Q_{W}\right)$ of ranks 2 and 1 respectively. If $\left\{l, l^{-1}\right\}$ is a basis of $E$, the Higgs field $\varphi$ of the $\mathrm{SO}_{0}(2,1)$-Higgs bundle and the orthogonal form $Q_{F}$ in the basis $\left\{l^{2}, l^{-2}, 1\right\}$ of $E=V \oplus W$ are of the form

$$
\varphi=\left(\begin{array}{ll|l} 
& & b \\
& & c \\
\hline 2 c & 2 b &
\end{array}\right) \text { and } Q_{F}=\left(\begin{array}{ll}
Q_{V} & \\
& Q_{W}
\end{array}\right)=\left(\begin{array}{ll|l}
2 & \\
2 & \\
\hline & & -1
\end{array}\right)
$$

We thus have the map

$$
\begin{aligned}
\mathcal{M}(\mathrm{SL}(2, \mathbb{R})) & \rightarrow \mathcal{M}\left(\mathrm{SO}_{0}(2,1)\right) \\
\left(L \oplus L^{-1}, \phi=\left(\begin{array}{ll}
0 & b \\
c & 0
\end{array}\right)\right) & \mapsto\left(V=L^{2} \oplus L^{-2}, W=\mathcal{O}, \eta=\left(\begin{array}{l}
b \\
c
\end{array}\right)\right) .
\end{aligned}
$$

Observe that, to obtain the Higgs field $\eta$, all we have to do is to consider the sections $b \in H^{0}\left(L^{2} \otimes K\right)$ and $c \in H^{0}\left(L^{-2} \otimes K\right)$ as homomorphisms from $\mathcal{O}$ to $L^{2} \otimes K$ and $L^{-2} \otimes K$ respectively.

As in the previous cases, if $\left(V=M \oplus M^{-1}, W=\mathcal{O}, \eta\right)$ is an $\mathrm{SO}_{0}(2,1)$-Higgs bundle, it lifts to an $\operatorname{SL}(2, \mathbb{R})$-Higgs bundle if and only if $\omega_{2}\left(F_{\mathrm{SO}(3)}\right)=0$, where $F=V \oplus W$. Since

$$
\omega_{2}\left(F_{\mathrm{SO}(3)}\right)=\operatorname{deg}(M) \bmod 2,
$$

it happens if and only if $\operatorname{deg}(M)$ is even.

\subsection{Isomorphisms of $\mathrm{SO}(4, \mathbb{C})$ and its real forms}

Since we have the isomorphism $\mathfrak{s o}(4, \mathbb{C}) \cong \mathfrak{s l}(2, \mathbb{C}) \times \mathfrak{s l}(2, \mathbb{C})$, the orthogonal Lie group $\mathrm{SO}(4, \mathbb{C})$ is locally isomorphic to $\operatorname{SL}(2, \mathbb{C}) \times \operatorname{SL}(2, \mathbb{C})$, but as in the previous section, the Lie group with Lie algebra $\mathfrak{s o}(4, \mathbb{C})$ which is isomorphic to $\operatorname{SL}(2, \mathbb{C}) \times \operatorname{SL}(2, \mathbb{C})$ is the spin 
group $\operatorname{Spin}(4, \mathbb{C})$. (See [28], Theorem 14.1). We have also local isomorphisms between the real forms

$$
\begin{aligned}
\mathrm{SO}(4) & \cong \mathrm{SU}(2) \times \mathrm{SU}(2), \\
\mathrm{SO}_{0}(2,2) & \cong \mathrm{SL}(2, \mathbb{R}) \times \mathrm{SL}(2, \mathbb{R}), \\
\mathrm{SO}_{0}(1,3) & \cong \mathrm{SL}(2, \mathbb{C}),
\end{aligned}
$$

that we will use to establish the relations between the corresponding moduli spaces of Higgs bundles.

For the compact real form $\mathrm{SO}(4)$ we have the sequence

$$
1 \rightarrow \mathbb{Z}_{2} \rightarrow \mathrm{Spin}(4) \cong \mathrm{SU}(2) \times \mathrm{SU}(2) \rightarrow \mathrm{SO}(4) \rightarrow 1
$$

Consider a pair of holomorphic vector bundles $E$ and $E^{\prime}$ of rank 2 and trivial determinant and let $F=E \otimes E^{\prime}$. This vector bundle has rank 4 and a non-degenerate symmetric quadratic form defined by

$$
\begin{aligned}
Q_{F}:\left(E \otimes E^{\prime}\right) \times\left(E \otimes E^{\prime}\right) & \rightarrow \Lambda^{2} E \otimes \Lambda^{2} E^{\prime}=\mathcal{O} \\
\left(x \otimes x^{\prime}, y \otimes y^{\prime}\right) & \mapsto(x \wedge y) \otimes\left(x^{\prime} \wedge y^{\prime}\right)
\end{aligned}
$$

We can define the map

$$
\begin{aligned}
\mathcal{M}(\mathrm{SU}(2) \times \mathrm{SU}(2)) & \rightarrow \mathcal{M}(\mathrm{SO}(4)) \\
\left(E, E^{\prime}\right) & \mapsto\left(F=E \otimes E^{\prime}, Q_{F}\right)
\end{aligned}
$$

A principal $\mathrm{SO}(4, \mathbb{C})$-bundle $\left(F, Q_{F}\right)$ lifts to a principal $\operatorname{Spin}(4, \mathbb{C})$-bundle if and only if $\omega_{2}\left(F_{\mathrm{SO}(4)}\right)=0 \in H^{2}\left(\mathbb{Z}_{2}\right) \cong \mathbb{Z}_{2}$.

Consider now the sequence for the complex group

$$
1 \rightarrow \mathbb{Z}_{2} \rightarrow \operatorname{Spin}(4, \mathbb{C}) \cong \mathrm{SL}(2, \mathbb{C}) \times \mathrm{SL}(2, \mathbb{C}) \rightarrow \mathrm{SO}(4, \mathbb{C}) \rightarrow 1
$$

and let $(E, \phi)$ and $\left(E^{\prime}, \phi^{\prime}\right)$, where $E$ and $E^{\prime}$ are holomorphic vector bundles of rank 2 and trivial determinant, $\phi \in H^{0}\left(\operatorname{End}_{0}(E) \otimes K\right)$ and $\phi^{\prime} \in H^{0}\left(\operatorname{End}_{0}\left(E^{\prime}\right) \otimes K\right)$, be a pair of $\mathrm{SL}(2, \mathbb{C})$-Higgs bundles. With the same construction as in the compact case, we obtain the principal $\mathrm{SO}(4, \mathbb{C})$-bundle $\left(F=E \otimes E^{\prime}, Q_{F}\right)$.

If $\left\{e_{1}, e_{2}\right\}$ and $\left\{e_{1}^{\prime}, e_{2}^{\prime}\right\}$ are basis of $E$ and $E^{\prime}$ respectively, then $\left\{e_{1} \otimes e_{1}^{\prime}, e_{1} \otimes e_{2}^{\prime}, e_{2} \otimes\right.$ $\left.e_{1}^{\prime}, e_{2} \otimes e_{2}^{\prime}\right\}$ is a basis of $F$. If

$$
\phi=\left(\begin{array}{cc}
\phi_{1,1} & \phi_{1,2} \\
\phi_{2,1} & -\phi_{1,1}
\end{array}\right) \quad \text { and } \quad \phi^{\prime}=\left(\begin{array}{cc}
\phi_{1,1}^{\prime} & \phi_{1,2}^{\prime} \\
\phi_{2,1}^{\prime} & -\phi_{1,1}^{\prime}
\end{array}\right)
$$


in the basis of $E$ and $E^{\prime}$ respectively, we obtain the following matrix for the Higgs field $\varphi$ of the $\mathrm{SO}(4, \mathbb{C})$-Higgs bundle in the basis of $F$,

$$
\varphi=\left(\begin{array}{cccc}
\phi_{1,1}+\phi_{1,1}^{\prime} & \phi_{1,2}^{\prime} & \phi_{1,2} & 0 \\
\phi_{2,1}^{\prime} & \phi_{1,1}-\phi_{1,1}^{\prime} & 0 & \phi_{1,2} \\
\phi_{2,1} & 0 & -\phi_{1,1}+\phi_{1,1}^{\prime} & \phi_{1,2}^{\prime} \\
0 & \phi_{2,1} & \phi_{2,1}^{\prime} & -\phi_{1,1}-\phi_{1,1}^{\prime}
\end{array}\right)
$$

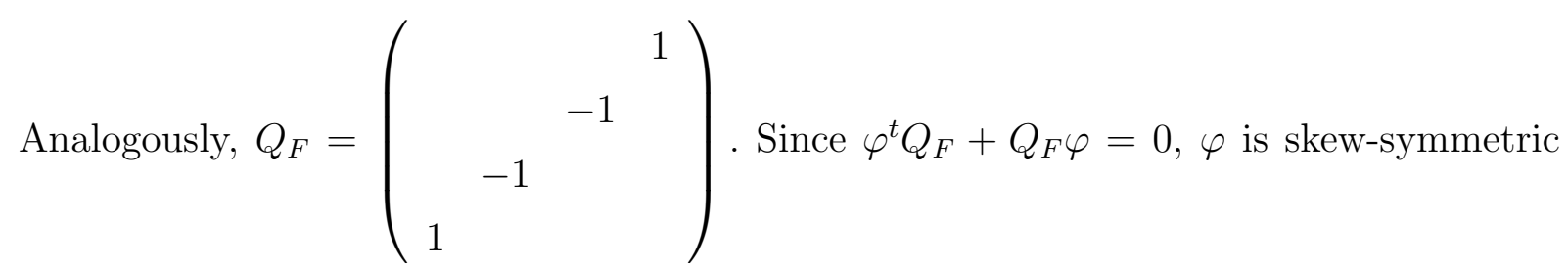
and we can finally establish the map

$$
\begin{aligned}
\mathcal{M}(\mathrm{SL}(2, \mathbb{C}) \times \mathrm{SL}(2, \mathbb{C})) & \rightarrow \mathcal{M}(\mathrm{SO}(4, \mathbb{C})) \\
(E, \phi),\left(E^{\prime}, \phi^{\prime}\right) & \mapsto\left(F=E \otimes E^{\prime}, Q_{F}, \varphi\right) .
\end{aligned}
$$

For the split real form $\mathrm{SO}_{0}(2,2)$ we have the following sequence

$$
1 \rightarrow \mathbb{Z}_{2} \rightarrow \operatorname{Spin}_{0}(2,2) \cong \mathrm{SL}(2, \mathbb{R}) \times \mathrm{SL}(2, \mathbb{R}) \rightarrow \mathrm{SO}_{0}(2,2) \rightarrow 1 .
$$

A pair of $\mathrm{SL}(2, \mathbb{R})$-Higgs bundles is a pair of triples

$$
\left(E=L \oplus L^{-1}, Q, \phi\right) \quad \text { and } \quad\left(E^{\prime}=M \oplus M^{-1}, Q^{\prime}, \phi^{\prime}\right),
$$

where $L$ and $M$ are holomorphic line bundles, $Q$ and $Q^{\prime}$ are the corresponding dual pairings and the Higgs fields $\phi$ and $\phi^{\prime}$ are symmetric trace-free endomorphisms of $E$ and $E^{\prime}$ respectively. In this case we have

$$
F=E \otimes E^{\prime}=(L \otimes M) \oplus\left(L \otimes M^{-1}\right) \oplus\left(L^{-1} \otimes M\right) \oplus\left(L^{-1} \otimes M^{-1}\right) .
$$

Taking $V=(L \otimes M) \oplus\left(L^{-1} \otimes M^{-1}\right)$ and $W=\left(L \otimes M^{-1}\right) \oplus\left(L^{-1} \otimes M\right)$, since $Q_{F}$ restricts to $V$ and $W$, we obtain the principal $\mathrm{SO}(2, \mathbb{C})$-bundles $\left(V, Q_{V}\right)$ and $\left(W, Q_{W}\right)$.

If $\left\{l, l^{-1}\right\}$ and $\left\{m, m^{-1}\right\}$ are basis of $E$ and $E^{\prime}$ respectively and

$$
\phi=\left(\begin{array}{cc} 
& \phi_{1,2} \\
\phi_{2,1} &
\end{array}\right) \quad \text { and } \quad \phi^{\prime}=\left(\begin{array}{cc} 
& \phi_{1,2}^{\prime} \\
\phi_{2,1}^{\prime} &
\end{array}\right),
$$

in these basis, the Higgs field $\varphi$ of the $\mathrm{SO}_{0}(2,2)$-Higgs bundle and the orthogonal form $Q_{F}$ in the basis $\left\{l \otimes m, l^{-1} \otimes m^{-1}, l \otimes m^{-1}, l^{-1} \otimes m\right\}$ of $F=V \oplus W$ are of the form

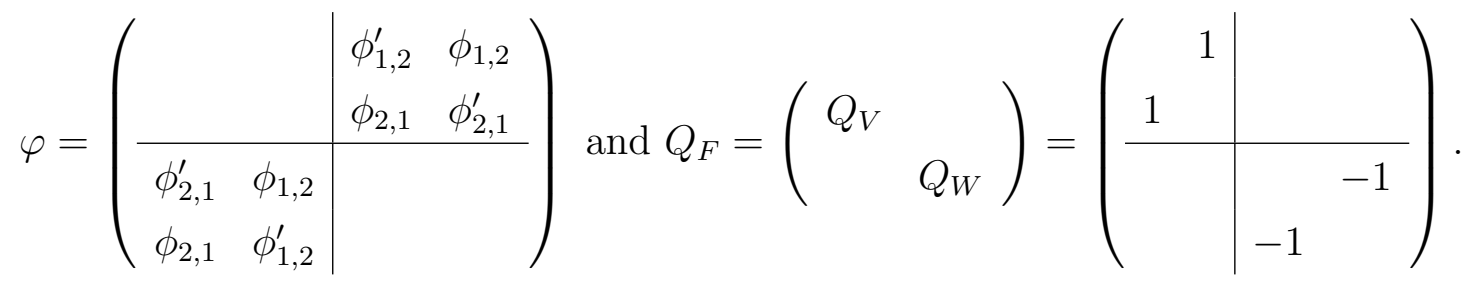


Then, the map

$$
\mathcal{M}(\mathrm{SL}(2, \mathbb{R}) \times \mathrm{SL}(2, \mathbb{R})) \rightarrow \mathcal{M}\left(\mathrm{SO}_{0}(2,2)\right)
$$

is given by

$$
\begin{gathered}
\left(L \oplus L^{-1}, \phi=\left(\begin{array}{ll} 
& \phi_{1,2} \\
\phi_{2,1} &
\end{array}\right), M \oplus M^{-1}, \phi^{\prime}=\left(\begin{array}{cc} 
& \phi_{1,2}^{\prime} \\
\phi_{2,1}^{\prime}
\end{array}\right)\right) \\
\downarrow \\
\left(V=(L \otimes M) \oplus\left(L^{-1} \otimes M^{-1}\right), W=\left(L \otimes M^{-1}\right) \oplus\left(L^{-1} \otimes M\right), \eta=\left(\begin{array}{ll}
\phi_{1,2}^{\prime} & \phi_{1,2} \\
\phi_{2,1} & \phi_{2,1}^{\prime}
\end{array}\right)\right) .
\end{gathered}
$$

For the real form $\mathrm{SO}_{0}(1,3)$ we have the following sequence

$$
1 \rightarrow \mathbb{Z}_{2} \rightarrow \operatorname{Spin}_{0}(1,3) \cong \mathrm{SL}(2, \mathbb{C}) \rightarrow \mathrm{SO}_{0}(1,3) \rightarrow 1
$$

If $E$ is a holomorphic vector bundle of rank 2 and trivial determinant and $\phi$ is a trace-free endomorphism of $E$ and we consider in $F=E \otimes E^{*}$ the quadratic form

$$
\begin{aligned}
Q_{F}:\left(E \otimes E^{*}\right) \times\left(E \otimes E^{*}\right) & \rightarrow \Lambda^{2} E \otimes \Lambda^{2} E^{*}=\mathcal{O} \\
\left(x \otimes x^{\prime}, y \otimes y^{\prime}\right) & \mapsto(x \wedge y) \otimes\left(x^{\prime} \wedge y^{\prime}\right),
\end{aligned}
$$

we obtain a principal $\mathrm{SO}(4, \mathbb{C})$-bundle.

If $\left\{e_{1}, e_{2}\right\}$ and $\left\{e_{1}^{-1}, e_{2}^{-1}\right\}$ are basis of $E$ and $E^{*}$ respectively, then $\left\{e_{1} \otimes e_{1}^{-1}+e_{2} \otimes\right.$ $\left.e_{2}^{-1}, e_{1} \otimes e_{2}^{-1}, e_{1} \otimes e_{1}^{-1}-e_{2} \otimes e_{2}^{-1}, e_{2} \otimes e_{1}^{-1}\right\}$ is a basis of $E \otimes E^{*}$. Let

$$
\begin{gathered}
V=\left\langle e_{1} \otimes e_{1}^{-1}+e_{2} \otimes e_{2}^{-1}\right\rangle, \\
W=\left\langle e_{1} \otimes e_{2}^{-1}, e_{1} \otimes e_{1}^{-1}-e_{2} \otimes e_{2}^{-1}, e_{2} \otimes e_{1}^{-1}\right\rangle .
\end{gathered}
$$

If $\phi=\left(\begin{array}{cc}a & b \\ c & -a\end{array}\right)$ in the basis of $E$ and $\phi^{t}=\left(\begin{array}{cc}a & c \\ b & -a\end{array}\right)$ in the corresponding basis of $E^{*}$, the Higgs field $\varphi$ of the $\mathrm{SO}_{0}(1,3)$-Higgs bundle and the orthogonal form $Q_{F}$ in the basis of $F=V \oplus W$ are of the form

$$
\varphi=\left(\begin{array}{rr} 
& \eta \\
-\eta^{\top} &
\end{array}\right)=\left(\begin{array}{cccc} 
& c & 2 a & b \\
\hline 2 b & & & \\
2 a & & & \\
2 c & &
\end{array}\right)
$$

and

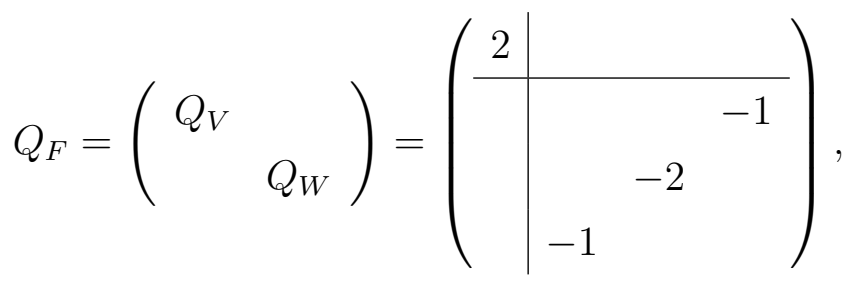


and we can define the map

$$
\begin{aligned}
\mathcal{M}(\mathrm{SL}(2, \mathbb{C})) & \rightarrow \mathcal{M}\left(\mathrm{SO}_{0}(1,3)\right) \\
(E, \phi) & \mapsto\left(F=E \otimes E^{*}, Q_{F}, \eta\right)
\end{aligned}
$$

\subsection{Isomorphisms of $\mathrm{SO}(5, \mathbb{C})$ and its real forms}

As in the previous cases, the isomorphisms

$$
\begin{aligned}
\operatorname{Spin}(5, \mathbb{C}) & \cong \operatorname{Sp}(4, \mathbb{C}), \\
\operatorname{Spin}(5) & \cong \operatorname{Sp}(4), \\
\operatorname{Spin}_{0}(2,3) & \cong \operatorname{Sp}(4, \mathbb{R}), \\
\operatorname{Spin}_{0}(1,4) & \cong \operatorname{Sp}(2,2),
\end{aligned}
$$

(see [28, Theorem 14.1]), and the fact that spin groups are double covers of the orthogonal groups, allow us, in each of the four cases, to define a map from the moduli space of symplectic Higgs bundles to the moduli space of special orthogonal Higgs bundles.

For the compact real form $\mathrm{SO}(5)$ we have the sequence

$$
1 \rightarrow \mathbb{Z}_{2} \rightarrow \operatorname{Spin}(5) \cong \mathrm{Sp}(4) \rightarrow \mathrm{SO}(5) \rightarrow 1
$$

an $\operatorname{Sp}(4)$-Higgs bundle is a principal $\operatorname{Sp}(4, \mathbb{C})$-bundle, that is, a holomorphic vector bundle $E$ of rank 4 and trivial determinant together with a non-degenerate antisymmetric quadratic form $\Omega \in H^{0}\left(\Lambda^{2} E^{*}\right)$. The representation $\Lambda^{2} V$ of $\operatorname{Sp}(4, \mathbb{C})$, where $V$ denotes the standard representation, decomposes as

$$
\Lambda^{2} V=W \oplus \mathbb{C}
$$

where $W$ is the irreducible five-dimensional representation of $\operatorname{Sp}(4, \mathbb{C})$ (See [18, §16.2]). Then, the vector bundle $\Lambda^{2} E$, which has rank 6 , decomposes as

$$
\Lambda^{2} E=F \oplus \mathcal{O}
$$

where $F$ is holomorphic vector bundle of rank 5 . In $\Lambda^{2} E$ we can define a non-degenerate symmetric quadratic form given by

$$
\begin{aligned}
Q: \Lambda^{2} E \times \Lambda^{2} E & \rightarrow \Lambda^{4} E=\mathcal{O} \\
\left(x \wedge y, x^{\prime} \wedge y^{\prime}\right) & \mapsto x \wedge y \wedge x^{\prime} \wedge y^{\prime}
\end{aligned}
$$


Then, $\left(\Lambda^{2} E, Q\right)$ is a principal $\mathrm{SO}(6, \mathbb{C})$-bundle. If $Q_{F}$ is the restriction of the orthogonal form $Q$ to $F,\left(F, Q_{F}\right)$ is a principal $\mathrm{SO}(5, \mathbb{C})$-bundle $\left(F, Q_{F}\right)$ and we can finally define the map

$$
\begin{aligned}
\mathcal{M}(\mathrm{Sp}(4)) & \rightarrow \mathcal{M}(\mathrm{SO}(5)) \\
(E, \Omega) & \mapsto\left(F, Q_{F}\right)
\end{aligned}
$$

Consider now the sequence for the complex group

$$
1 \rightarrow \mathbb{Z}_{2} \rightarrow \operatorname{Spin}(5, \mathbb{C}) \cong \operatorname{Sp}(4, \mathbb{C}) \rightarrow \mathrm{SO}(5, \mathbb{C}) \rightarrow 1
$$

an $\operatorname{Sp}(4, \mathbb{C})$-Higgs bundle is a triple $(E, \Omega, \phi)$ consisting of a holomorphic vector bundle $E$ of rank 4 and trivial determinant, a symplectic form $\Omega$ on $E$ and a holomorphic section $\phi \in H^{0}(\mathfrak{s p}(E) \otimes K)$, where

$$
\mathfrak{s p}(F)=\left\{f \in \operatorname{End}_{0}(E) \mid \Omega(f \cdot, \cdot)+\Omega(\cdot, f \cdot)=0\right\} .
$$

If $\left\{e_{1}, e_{2}, e_{3}, e_{4}\right\}$ is a basis of $E$, then $\left\{e_{1} \wedge e_{2}, e_{1} \wedge e_{3}, e_{1} \wedge e_{4}, e_{2} \wedge e_{3}, e_{2} \wedge e_{4}, e_{3} \wedge e_{4}\right\}$ is a basis of $\Lambda^{2} E=F \oplus \mathcal{O}$. The matrix of $Q$ in this basis is

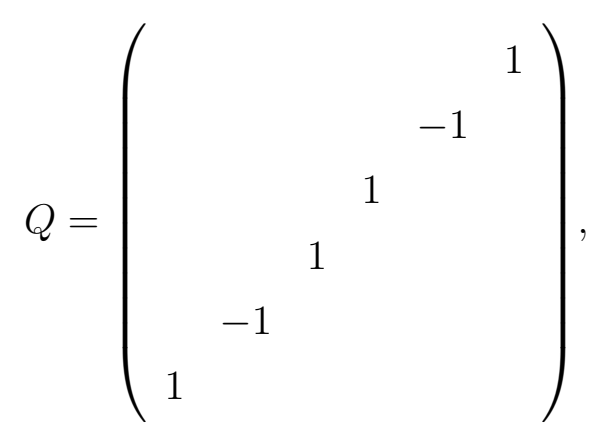

(where we are considering $e_{1} \wedge e_{2} \wedge e_{3} \wedge e_{4}=1$ under the isomorphism $\Lambda^{4} E \cong \mathcal{O}$ ). On the other hand, if

in the basis of $E$, (using $\Omega=\left(\begin{array}{ll|ll} & 1 & \\ & & 1 \\ \hline-1 & & & \\ & -1 & & \end{array}\right)$ ), we obtain the matrix of the Higgs field of the $\mathrm{SO}(6, \mathbb{C})$-Higgs bundle $\left(\Lambda^{2} E, Q, \varphi\right)$ in the corresponding basis of $\Lambda^{2} E$ computing

$$
\varphi\left(e_{i} \wedge e_{j}\right)=\phi\left(e_{i}\right) \wedge e_{j}+e_{i} \wedge \phi\left(e_{j}\right)
$$


The resulting matrix is

$$
\varphi=\left(\begin{array}{cccccc}
\phi_{1,1}+\phi_{2,2} & \phi_{1,4} & \phi_{2,4} & -\phi_{1,3} & -\phi_{1,4} & 0 \\
\phi_{3,2} & 0 & -\phi_{2,1} & \phi_{1,2} & 0 & -\phi_{1,4} \\
\phi_{4,2} & -\phi_{1,2} & \phi_{1,1}-\phi_{2,2} & 0 & \phi_{1,2} & \phi_{1,3} \\
-\phi_{3,1} & \phi_{2,1} & 0 & \phi_{2,2}-\phi_{1,1} & -\phi_{2,1} & -\phi_{2,4} \\
-\phi_{3,2} & 0 & \phi_{2,1} & -\phi_{1,2} & 0 & \phi_{1,4} \\
0 & -\phi_{3,2} & \phi_{3,1} & -\phi_{4,2} & \phi_{3,2} & -\phi_{1,1}-\phi_{2,2}
\end{array}\right) .
$$

Observe that $\varphi\left(e_{1} \wedge e_{3}+e_{2} \wedge e_{4}\right)=0$. Hence, we can set $F=\left\langle e_{1} \wedge e_{2}, e_{1} \wedge e_{4}, e_{1} \wedge e_{3}-\right.$ $\left.e_{2} \wedge e_{4}, e_{2} \wedge e_{3}, e_{3} \wedge e_{4}\right\rangle$. The matrix of the orthogonal form $Q_{F}$ in this basis of $F$ is

$$
Q_{F}=\left(\begin{array}{cccc} 
& & & 1 \\
& & 1 & \\
& 2 & \\
& & & \\
1 & & &
\end{array}\right)
$$

and the matrix of the Higgs field of the $\mathrm{SO}(5, \mathbb{C})$-Higgs bundle $\left(F, Q_{F}, \varphi_{F}\right)$ is

$$
\varphi_{F}=\left(\begin{array}{ccccc}
\phi_{1,1}+\phi_{2,2} & \phi_{2,4} & 2 \phi_{1,4} & -\phi_{1,3} & 0 \\
\phi_{4,2} & \phi_{1,1}-\phi_{2,2} & -2 \phi_{1,2} & 0 & \phi_{1,3} \\
\phi_{3,2} & -\phi_{2,1} & 0 & \phi_{1,2} & -\phi_{1,4} \\
-\phi_{3,1} & 0 & 2 \phi_{2,1} & \phi_{2,2}-\phi_{1,1} & -\phi_{2,4} \\
0 & \phi_{3,1} & -2 \phi_{3,2} & -\phi_{4,2} & -\phi_{1,1}-\phi_{2,2}
\end{array}\right) \text {, }
$$

which satisfies $\varphi_{F}^{t} Q_{F}+Q_{F} \varphi_{F}=0$. We can establish the map

$$
\begin{aligned}
\mathcal{M}(\operatorname{Sp}(4, \mathbb{C})) & \rightarrow \mathcal{M}(\operatorname{SO}(5, \mathbb{C})) \\
(E, \Omega, \phi) & \mapsto\left(F, Q_{F}, \varphi_{F}\right)
\end{aligned}
$$

For the split real form $\mathrm{SO}_{0}(2,3)$ we have the following sequence

$$
1 \rightarrow \mathbb{Z}_{2} \rightarrow \operatorname{Spin}_{0}(2,3) \cong \mathrm{Sp}(4, \mathbb{R}) \rightarrow \mathrm{SO}_{0}(2,3) \rightarrow 1
$$

an $\operatorname{Sp}(4, \mathbb{R})$-Higgs bundle is a triple $(E, \beta, \gamma)$, where $E$ is a holomorphic vector bundle of rank $2, \beta \in H^{0}\left(S^{2} E \otimes K\right)$ and $\gamma \in H^{0}\left(S^{2} E^{*} \otimes K\right)$. In this case, we have

$$
\Lambda^{2}\left(E \oplus E^{*}\right)=\Lambda^{2} E \oplus\left(E \otimes E^{*}\right) \oplus \Lambda^{2} E^{*}=F \oplus \mathcal{O}=V \oplus W \oplus \mathcal{O}
$$

If $\left\{e_{1}, e_{2}\right\}$ is a basis of $E$, then $\left\{e_{1} \wedge e_{2}, e_{1} \wedge e_{1}^{-1}, e_{1} \wedge e_{2}^{-1}, e_{2} \wedge e_{1}^{-1}, e_{2} \wedge e_{2}^{-1}, e_{1}^{-1} \wedge e_{2}^{-1}\right\}$ is a basis of $\Lambda^{2}\left(E \oplus E^{*}\right)$ and if

$$
\beta=\left(\begin{array}{ll}
\beta_{1} & \beta_{2} \\
\beta_{2} & \beta_{3}
\end{array}\right) \quad \text { and } \quad \gamma=\left(\begin{array}{ll}
\gamma_{1} & \gamma_{2} \\
\gamma_{2} & \gamma_{3}
\end{array}\right)
$$


in the basis of $E$ and $E^{*}$, the matrix of the Higgs field $\varphi$ in this basis is

$$
\varphi=\left(\begin{array}{cccccc} 
& \beta_{2} & \beta_{3} & -\beta_{1} & -\beta_{2} & \\
\gamma_{2} & & & & & -\beta_{2} \\
\gamma_{3} & & & & & \beta_{1} \\
-\gamma_{1} & & & & & -\beta_{3} \\
-\gamma_{2} & & & & & \beta_{2} \\
& -\gamma_{2} & \gamma_{1} & -\gamma_{3} & \gamma_{2} &
\end{array}\right) .
$$

Again, since $\varphi\left(e_{1} \wedge e_{1}^{-1}+e_{2} \wedge e_{2}^{-1}\right)=0$, we set $F=\left\langle e_{1} \wedge e_{2}, e_{1} \wedge e_{2}^{-1}, e_{1} \wedge e_{1}^{-1}-e_{2} \wedge e_{2}^{-1}, e_{2} \wedge\right.$ $\left.e_{1}^{-1}, e_{1}^{-1} \wedge e_{2}^{-1}\right\rangle$. Let

$$
\begin{gathered}
V=\left\langle e_{1} \wedge e_{2}, e_{1}^{-1} \wedge e_{2}^{-1}\right\rangle=\Lambda^{2} E \oplus \Lambda^{2} E^{*} \\
W=\left\langle e_{1} \wedge e_{2}^{-1}, e_{1} \wedge e_{1}^{-1}-e_{2} \wedge e_{2}^{-1}, e_{2} \wedge e_{1}^{-1}\right\rangle \subset E \otimes E^{*} .
\end{gathered}
$$

We can define the orthogonal forms $Q$ and $Q_{F}$ as in the previous cases because $\Lambda^{4}(E \oplus$ $\left.E^{*}\right)=\Lambda^{2} E \otimes\left(\Lambda^{2} E\right)^{-1}=\mathcal{O}$. The matrix of $Q_{F}$ in this basis is

$$
Q_{F}=\left(\begin{array}{ll}
Q_{V} & \\
& Q_{W}
\end{array}\right)=\left(\begin{array}{ll|ll}
1 & & \\
1 & & & \\
\hline & & & \\
& & 2 & \\
& & &
\end{array}\right)
$$

and the matrix of the Higgs field is

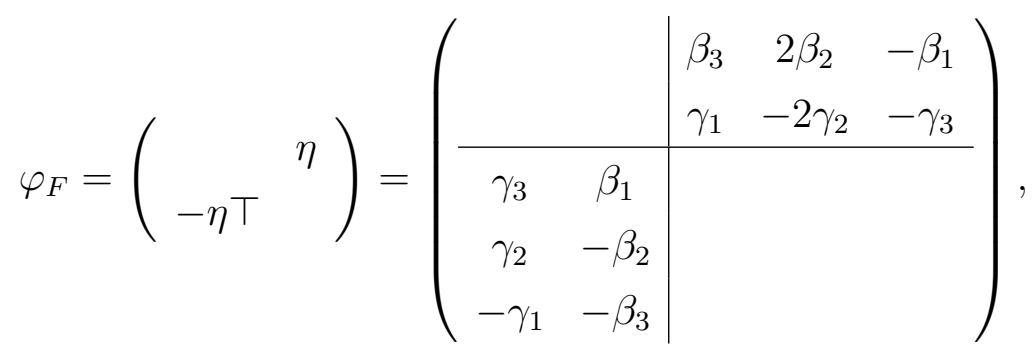

which satisfies $\varphi_{F}^{t} Q_{F}+Q_{F} \varphi_{F}=0$. We can establish the map

$$
\begin{aligned}
\mathcal{M}(\mathrm{Sp}(4, \mathbb{R})) & \rightarrow \mathcal{M}\left(\mathrm{SO}_{0}(2,3)\right) \\
(E, \beta, \gamma) & \mapsto\left(V=\Lambda^{2} E \oplus \Lambda^{2} E^{*}, Q_{V}, W \subset E \otimes E^{*}, Q_{W}, \eta\right) .
\end{aligned}
$$

For the real form $\mathrm{SO}_{0}(1,4)$ we have the following sequence

$$
1 \rightarrow \mathbb{Z}_{2} \rightarrow \operatorname{Spin}_{0}(1,4) \cong \operatorname{Sp}(2,2) \rightarrow \mathrm{SO}_{0}(1,4) \rightarrow 1
$$


Using the Cartan decomposition (see [29, Ch. X, §2]),

$$
\mathfrak{s p}(2,2)=\mathfrak{s p}(2) \times \mathfrak{s p}(2) \oplus \mathfrak{m},
$$

where

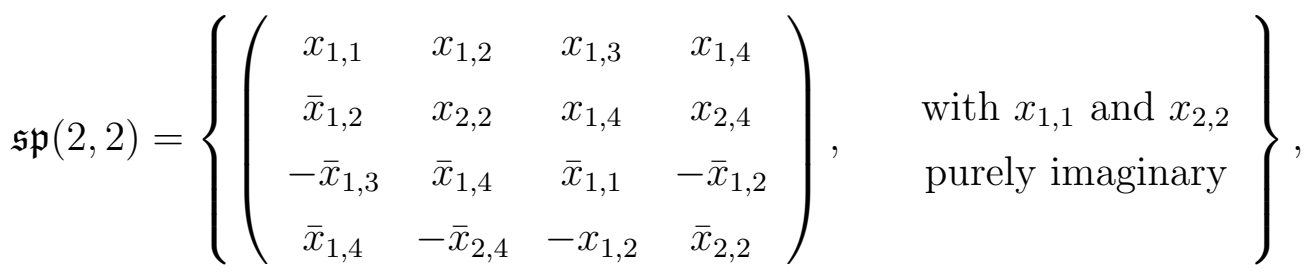

and

$$
\mathfrak{m}=\left\{\left(\begin{array}{cccc} 
& i x_{1,2} & & i x_{1,4} \\
-i \bar{x}_{1,2} & & i x_{1,4} & \\
& -i \bar{x}_{1,4} & & i \bar{x}_{1,2} \\
-i \bar{x}_{1,4} & & -i x_{1,2}
\end{array}\right)\right\},
$$

and the imbedding of $\mathfrak{s p}(2) \times \mathfrak{s p}(2)$ in $\mathfrak{s p}(2,2)$ given by

$$
\left(\begin{array}{cc}
x_{1,1} & x_{1,3} \\
-\bar{x}_{1,3} & \bar{x}_{1,1}
\end{array}\right),\left(\begin{array}{cc}
x_{2,2} & x_{2,4} \\
-\bar{x}_{2,4} & \bar{x}_{2,2}
\end{array}\right) \mapsto\left(\begin{array}{cccc}
x_{1,1} & & x_{1,3} & \\
& x_{2,2} & & x_{2,4} \\
-\bar{x}_{1,3} & & \bar{x}_{1,1} & \\
& -\bar{x}_{2,4} & & \bar{x}_{2,2}
\end{array}\right),
$$

(for the dimensions, observe that $x_{1,1}$ and $x_{2,2}$ are purely imaginary), we can see an $\operatorname{Sp}(2,2)$-Higgs bundle as a tuple $\left(E, \Omega, E^{\prime}, \Omega^{\prime}, \phi\right)$, where $E$ and $E^{\prime}$ are holomorphic vector bundles of rank 2 and trivial determinant, $\Omega$ and $\Omega^{\prime}$ are symplectic forms on $E$ and $E^{\prime}$ respectively and the Higgs field is of the form $\phi=\left(\begin{array}{cccc} & a & & b \\ c & & b & \\ & d & & -c \\ d & & -a\end{array}\right)$. If $\left\{e_{1}, e_{2}\right\}$ and $\left\{e_{1}^{\prime}, e_{2}^{\prime}\right\}$ are basis of $E$ and $E^{\prime}$ respectively, the Higgs field $\phi$ is in the basis $\left\{e_{1}, e_{1}^{\prime}, e_{2}, e_{2}^{\prime}\right\}$. Now we have

$$
\Lambda^{2}\left(E \oplus E^{\prime}\right)=\Lambda^{2} E \oplus\left(E \otimes E^{\prime}\right) \oplus \Lambda^{2} E^{\prime}=\mathcal{O} \oplus\left(E \otimes E^{\prime}\right) \oplus \mathcal{O}=V \oplus W .
$$

In the basis $\left\{e_{1} \wedge e_{2}, e_{1} \wedge e_{1}^{\prime}, e_{1} \wedge e_{2}^{\prime}, e_{2} \wedge e_{1}^{\prime}, e_{2} \wedge e_{2}^{\prime}, e_{1}^{\prime} \wedge e_{2}^{\prime}\right\}$ of $\Lambda^{2}\left(E \oplus E^{\prime}\right)$, the matrix of the Higgs field is

$$
\varphi=\left(\begin{array}{cccccc} 
& d & -c & -a & -b & \\
b & & & & & -b \\
-a & & & & & a \\
-c & & & & & c \\
-d & & & & & d \\
& -d & c & a & b &
\end{array}\right) .
$$


Since $\varphi\left(e_{1} \wedge e_{2}+e_{1}^{\prime} \wedge e_{2}^{\prime}\right)=0$, we set $F=\left\langle e_{1} \wedge e_{1}^{\prime}, e_{1} \wedge e_{2}^{\prime}, e_{1} \wedge e_{2}-e_{1}^{\prime} \wedge e_{2}^{\prime}, e_{2} \wedge e_{1}^{\prime}, e_{2} \wedge e_{2}^{\prime}\right\rangle$. Let

$$
\begin{gathered}
V=\left\langle e_{1} \wedge e_{2}-e_{1}^{\prime} \wedge e_{2}^{\prime}\right\rangle=\mathcal{O}, \\
W=\left\langle e_{1} \wedge e_{1}^{\prime}, e_{1} \wedge e_{2}^{\prime}, e_{2} \wedge e_{1}^{\prime}, e_{2} \wedge e_{2}^{\prime}\right\rangle=E \otimes E^{\prime} .
\end{gathered}
$$

Since $\Lambda^{4}\left(E \oplus E^{\prime}\right)=\Lambda^{2} E \otimes \Lambda^{2} E^{\prime}=\mathcal{O} \otimes \mathcal{O}$, we can define the orthogonal forms $Q$ and $Q_{F}$ as in the previous cases. The matrix of the orthogonal form in this basis is

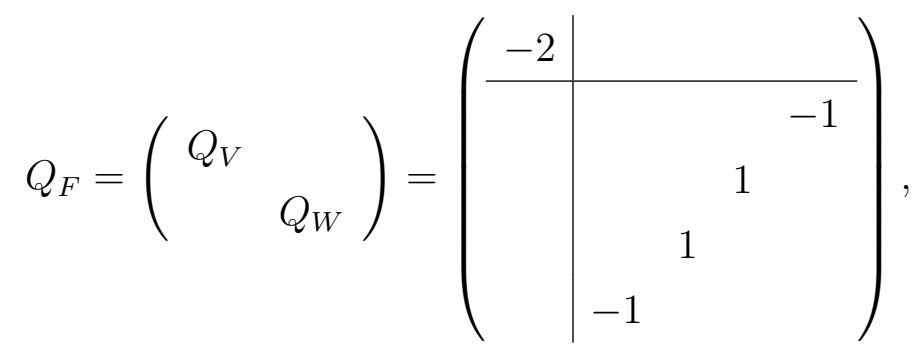

(fixing $e_{1} \wedge e_{2} \wedge e_{1}^{\prime} \wedge e_{2}^{\prime}=1$ ), and the matrix of the Higgs field is

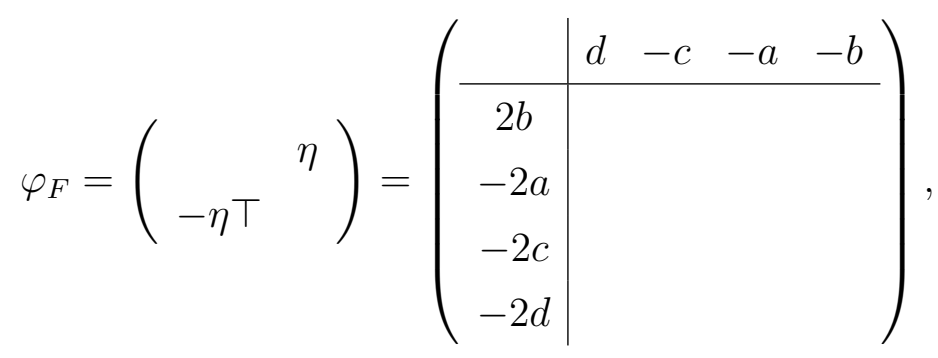

which satisfies $\varphi_{F}^{t} Q_{F}+Q_{F} \varphi_{F}=0$. We can finally define the map

$$
\begin{aligned}
\mathcal{M}(\mathrm{Sp}(2,2)) & \rightarrow \mathcal{M}\left(\mathrm{SO}_{0}(1,4)\right) \\
\left(E, \Omega, E^{\prime}, \Omega^{\prime}, \phi\right) & \mapsto\left(V=\mathcal{O}, Q_{V}, W=E \otimes E^{\prime}, Q_{W}, \eta\right) .
\end{aligned}
$$

\subsection{Isomorphisms of $\mathrm{SO}(6, \mathbb{C})$ and its real forms}

The Lie group $\mathrm{SO}(6, \mathbb{C})$ is locally isomorphic to the special linear group $\mathrm{SL}(4, \mathbb{C})$ and the spin group $\operatorname{Spin}(6, \mathbb{C})$ is isomorphic to $\operatorname{SL}(4, \mathbb{C})$ (see [28, Theorem 14.1]). Between the corresponding real forms we have the following isomorphisms:

$$
\begin{aligned}
\operatorname{Spin}(6) & \cong \mathrm{SU}(4), \\
\operatorname{SO}_{0}(3,3) & \cong \mathrm{SL}(4, \mathbb{R}), \\
\operatorname{Spin}_{0}(2,4) & \cong \mathrm{SU}(2,2), \\
\operatorname{Spin}_{0}(1,5) & \cong \mathrm{SU}^{*}(4) .
\end{aligned}
$$

We start with the compact real form $\mathrm{SO}(6)$ of $\mathrm{SO}(6, \mathbb{C})$. We have the sequence

$$
1 \rightarrow \mathbb{Z}_{2} \rightarrow \operatorname{Spin}(6) \cong \mathrm{SU}(4) \rightarrow \mathrm{SO}(6) \rightarrow 1
$$


If $E$ is a holomorphic vector bundle of rank 4 and trivial determinant, then $F=\Lambda^{2} E$ is a holomorphic vector bundle of rank 6 on which we can define a non-degenerate symmetric quadratic form

$$
\begin{aligned}
Q_{F}: \Lambda^{2} E \times \Lambda^{2} E & \rightarrow \Lambda^{4} E=\mathcal{O} \\
\left(x \wedge y, x^{\prime} \wedge y^{\prime}\right) & \mapsto x \wedge y \wedge x^{\prime} \wedge y^{\prime}
\end{aligned}
$$

obtaining a principal $\mathrm{SO}(6, \mathbb{C})$-bundle $\left(F, Q_{F}\right)$. Then, we can define the map

$$
\begin{aligned}
\mathcal{M}(\mathrm{SU}(4)) & \rightarrow \mathcal{M}(\mathrm{SO}(6)) \\
E & \mapsto\left(F=\Lambda^{2} E, Q_{F}\right)
\end{aligned}
$$

Consider now the sequence for the complex group

$$
1 \rightarrow \mathbb{Z}_{2} \rightarrow \operatorname{Spin}(6, \mathbb{C}) \cong \mathrm{SL}(4, \mathbb{C}) \rightarrow \mathrm{SO}(6, \mathbb{C}) \rightarrow 1
$$

Let $(E, \phi)$, where $E$ is a holomorphic vector bundle of rank 4 and trivial determinant and $\phi \in H^{0}\left(\operatorname{End}_{0}(E) \otimes K\right)$, be an $\operatorname{SL}(4, \mathbb{C})$-Higgs bundle. With the same construction as in the compact case, we obtain a principal $\mathrm{SO}(6, \mathbb{C})$-bundle $\left(F=\Lambda^{2} E, Q_{F}\right)$.

If $\left\{e_{1}, e_{2}, e_{3}, e_{4}\right\}$ is a basis of $E$, then $\left\{e_{1} \wedge e_{2}, e_{1} \wedge e_{3}, e_{1} \wedge e_{4}, e_{2} \wedge e_{3}, e_{2} \wedge e_{4}, e_{3} \wedge e_{4}\right\}$ is a basis of $F$. We obtain the matrix of the Higgs field $\varphi$ of the corresponding $\mathrm{SO}(6, \mathbb{C})$-Higgs bundle in the corresponding basis of $F$ computing

$$
\varphi\left(e_{i} \wedge e_{j}\right)=\phi\left(e_{i}\right) \wedge e_{j}+e_{i} \wedge \phi\left(e_{j}\right)
$$

It is a skew-symmetric endomorphism of $F$ and we can finally establish the correspondence

$$
\begin{aligned}
\mathcal{M}(\mathrm{SL}(4, \mathbb{C})) & \rightarrow \mathcal{M}(\mathrm{SO}(6, \mathbb{C})) \\
(E, \phi) & \mapsto\left(F, Q_{F}, \varphi\right) .
\end{aligned}
$$

For the split real form $\mathrm{SO}_{0}(3,3)$ we have an isomorphism

$$
\mathrm{SL}(4, \mathbb{R}) \stackrel{\cong}{\longrightarrow} \mathrm{SO}_{0}(3,3) .
$$

The correspondence between the moduli spaces of Higgs bundles is the following. We start with a principal $\mathrm{SO}(4, \mathbb{C})$-bundle $\left(E, Q_{E}\right)$ and a symmetric trace-free endomorphism $\phi$ of $E$. With the same construction as in the compact case, we obtain the principal $\mathrm{SO}(6, \mathbb{C})$-bundle $\left(F=\Lambda^{2} E, Q_{F}\right)$.

If $\left\{e_{1}, e_{2}, e_{3}, e_{4}\right\}$ is the basis of $E$, then $\left\{e_{1} \wedge e_{2}, e_{1} \wedge e_{4}+e_{2} \wedge e_{3}, e_{3} \wedge e_{4}, e_{1} \wedge e_{3}, e_{1} \wedge\right.$ $\left.e_{4}-e_{2} \wedge e_{3}, e_{2} \wedge e_{4}\right\}$ is a basis of $F$. Let

$$
V=\left\langle e_{1} \wedge e_{2}, e_{1} \wedge e_{4}+e_{2} \wedge e_{3}, e_{3} \wedge e_{4}\right\rangle,
$$




$$
W=\left\langle e_{1} \wedge e_{3}, e_{1} \wedge e_{4}-e_{2} \wedge e_{3}, e_{2} \wedge e_{4}\right\rangle
$$

The matrix of the orthogonal form in this basis is

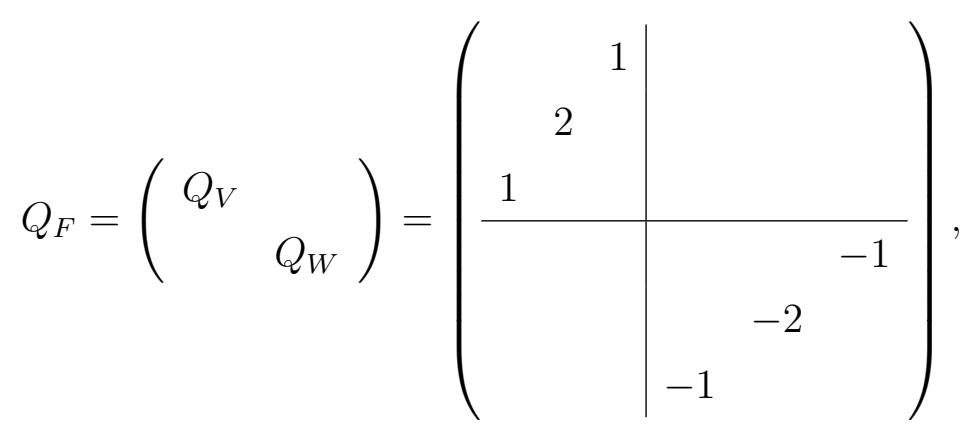

and if

$$
\phi=\left(\begin{array}{cccc}
\phi_{1,1} & \phi_{1,2} & \phi_{1,3} & \phi_{1,4} \\
\phi_{2,1} & -\phi_{1,1} & \phi_{2,3} & \phi_{1,3} \\
\phi_{3,1} & \phi_{3,2} & -\phi_{1,1} & \phi_{1,2} \\
\phi_{4,1} & \phi_{3,1} & \phi_{2,1} & \phi_{1,1}
\end{array}\right),
$$

in the basis of $E$, the matrix of the Higgs field $\varphi$ of the corresponding $\mathrm{SO}_{0}(3,3)$-Higgs bundle is

$$
\varphi=\left(\begin{array}{cc} 
& \eta \\
-\eta^{\top} &
\end{array}\right)=\left(\begin{array}{ccc|ccc} 
& & & \phi_{2,3} & 2 \phi_{1,3} & -\phi_{1,4} \\
& & & \phi_{2,1} & 2 \phi_{1,1} & \phi_{1,2} \\
& & & -\phi_{4,1} & 2 \phi_{3,1} & \phi_{3,2} \\
\hline \phi_{3,2} & 2 \phi_{1,2} & -\phi_{1,4} & & & \\
\phi_{3,1} & 2 \phi_{1,1} & \phi_{1,3} \\
-\phi_{4,1} & 2 \phi_{2,1} & \phi_{2,3} & & &
\end{array}\right),
$$

which satisfies $\varphi^{t} Q_{F}+Q_{F} \varphi=0$. We can establish the correspondence

$$
\begin{aligned}
\mathcal{M}(\mathrm{SL}(4, \mathbb{R})) & \rightarrow \mathcal{M}\left(\mathrm{SO}_{0}(3,3)\right) \\
(E, Q, \phi) & \mapsto\left(V, Q_{V}, W, Q_{W}, \eta\right)
\end{aligned}
$$

For the real form $\mathrm{SO}_{0}(2,4)$ we have the sequence

$$
1 \rightarrow \mathbb{Z}_{2} \rightarrow \operatorname{Spin}_{0}(2,4) \cong \mathrm{SU}(2,2) \rightarrow \mathrm{SO}_{0}(2,4) \rightarrow 1
$$

A SU(2,2)-Higgs bundle is a tuple $\left(E, E^{\prime}, \beta, \gamma\right)$ consisting of two holomorphic vector bundles $E$ and $E^{\prime}$ of rank 2 satisfying $\Lambda^{2} E \otimes \Lambda^{2} E^{\prime}=\mathcal{O}$ and two homomorphisms $\beta: E^{\prime} \rightarrow E \otimes K$ and $\gamma: E \rightarrow E^{\prime} \otimes K$. We consider

$$
\Lambda^{2}\left(E \oplus E^{\prime}\right)=\Lambda^{2} E \oplus\left(E \otimes E^{\prime}\right) \oplus \Lambda^{2} E^{\prime}
$$


and let $V=\Lambda^{2} E \oplus \Lambda^{2} E^{\prime}$ and $W=E \otimes E^{\prime}$.

If $\left\{e_{1}, e_{2}\right\}$ and $\left\{e_{1}^{\prime}, e_{2}^{\prime}\right\}$ are basis of $E$ and $E^{\prime}$ respectively, consider the basis $\left\{e_{1} \wedge\right.$ $\left.e_{2}, e_{1}^{\prime} \wedge e_{2}^{\prime}, e_{1} \wedge e_{1}^{\prime}, e_{1} \wedge e_{2}^{\prime}, e_{2} \wedge e_{1}^{\prime}, e_{2} \wedge e_{2}^{\prime}\right\}$ of $\Lambda^{2}\left(E \oplus E^{\prime}\right)=V \oplus W$. The matrix of the orthogonal form in this basis is

$$
Q_{F}=\left(\begin{array}{ll}
Q_{V} & \\
& Q_{W}
\end{array}\right)=\left(\begin{array}{cc|ccc} 
& 1 & & & \\
1 & & & & \\
\hline & & & & -1 \\
& & & 1 & \\
& & & & \\
& & & & \\
& & &
\end{array}\right),
$$

and if

$$
\beta=\left(\begin{array}{cc}
\beta_{1} & \beta_{2} \\
\beta_{3} & \beta_{4}
\end{array}\right) \quad \text { and } \quad \gamma=\left(\begin{array}{ll}
\gamma_{1} & \gamma_{2} \\
\gamma_{3} & \gamma_{4}
\end{array}\right)
$$

in the basis of $E$ and $E^{\prime}$, the matrix of the Higgs filed $\varphi$ of the corresponding $\operatorname{SO}_{0}(2,4)$ is

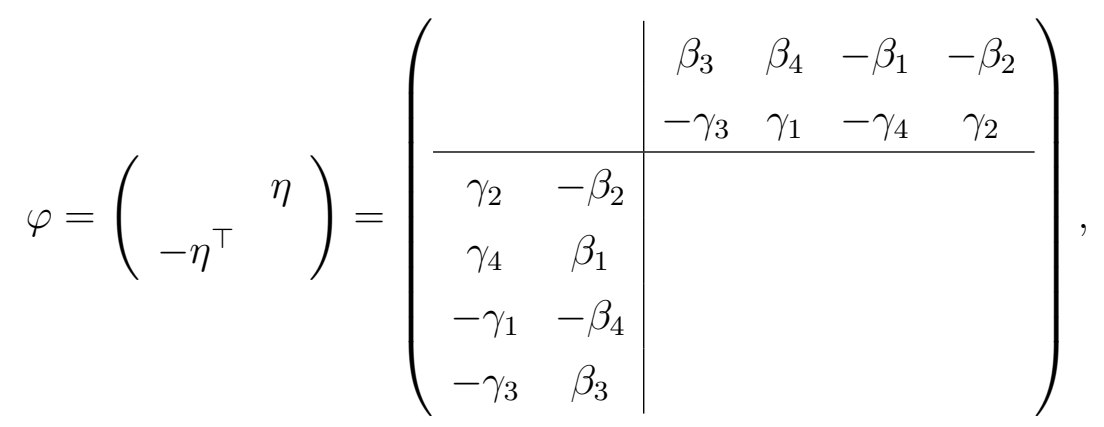

which satisfies $\varphi^{t} Q_{F}+Q_{F} \varphi=0$. We can define the map

$$
\begin{aligned}
\mathcal{M}(\mathrm{SU}(2,2)) & \rightarrow \mathcal{M}\left(\mathrm{SO}_{0}(2,4)\right) \\
\left(E, E^{\prime}, \beta, \gamma\right) & \mapsto\left(V, Q_{V}, W, Q_{W}, \eta\right)
\end{aligned}
$$

For the real form $\mathrm{SO}_{0}(1,5)$ we have the following sequence

$$
1 \rightarrow \mathbb{Z}_{2} \rightarrow \operatorname{Spin}_{0}(1,5) \cong \mathrm{SU}^{*}(4) \rightarrow \mathrm{SO}_{0}(1,5) \rightarrow 1
$$

Using the Cartan decomposition (see [29, Ch. X, §2]),

$$
\mathfrak{s} \mathfrak{u}^{*}(4)=\mathfrak{s p}(4) \oplus \mathfrak{m},
$$

where

$$
\mathfrak{s u}^{*}(4)=\left\{\left(\begin{array}{cc}
Z_{1} & Z_{2} \\
\bar{Z}_{2}^{t} & \bar{Z}_{1}
\end{array}\right) \mid \operatorname{Tr} Z_{1}+\operatorname{Tr} \bar{Z}_{1}=0\right\}
$$




$$
\mathfrak{m}=\left\{\left(\begin{array}{cc}
i Z_{1} & i Z_{2} \\
i \bar{Z}_{2} & -i \bar{Z}_{1}
\end{array}\right) \mid Z_{1} \in \mathfrak{s u}(2), Z_{2} \in \mathfrak{s o}(2, \mathbb{C})\right\}
$$

and

$$
\mathfrak{m}^{\mathbb{C}}=\left\{\left(\begin{array}{cc}
A & B \\
C & A^{t}
\end{array}\right) \mid \operatorname{Tr} A=0, B, C \in \mathfrak{s o}(2, \mathbb{C})\right\},
$$

we can see an $\operatorname{Sp}(2,2)$-Higgs bundle as a triple $(E, \Omega, \phi)$, where $E$ is a holomorphic vector bundles of rank 4 and trivial determinant, $\Omega$ is a symplectic form on $E$ and

$$
\phi=\left(\begin{array}{cc|cc}
\phi_{1,1} & \phi_{1,2} & 0 & \phi_{1,4} \\
\phi_{2,1} & -\phi_{1,1} & -\phi_{1,4} & 0 \\
\hline 0 & \phi_{3,2} & \phi_{1,1} & \phi_{2,1} \\
-\phi_{3,2} & 0 & \phi_{1,2} & -\phi_{1,1}
\end{array}\right) .
$$

If $\left\{e_{1}, e_{2}, e_{3}, e_{4}\right\}$ is a basis of $E$, consider the basis $\left\{e_{1} \wedge e_{3}+e_{2} \wedge e_{4}, e_{1} \wedge e_{2}, e_{1} \wedge e_{4}, e_{1} \wedge\right.$ $\left.e_{3}-e_{2} \wedge e_{4}, e_{2} \wedge e_{3}, e_{3} \wedge e_{4}\right\}$ of $F$ and let

$$
\begin{gathered}
V=\left\langle e_{1} \wedge e_{3}+e_{2} \wedge e_{4}\right\rangle, \\
W=\left\langle e_{1} \wedge e_{2}, e_{1} \wedge e_{4}, e_{1} \wedge e_{3}-e_{2} \wedge e_{4}, e_{2} \wedge e_{3}, e_{3} \wedge e_{4}\right\rangle .
\end{gathered}
$$

The matrix of the orthogonal form in this basis is

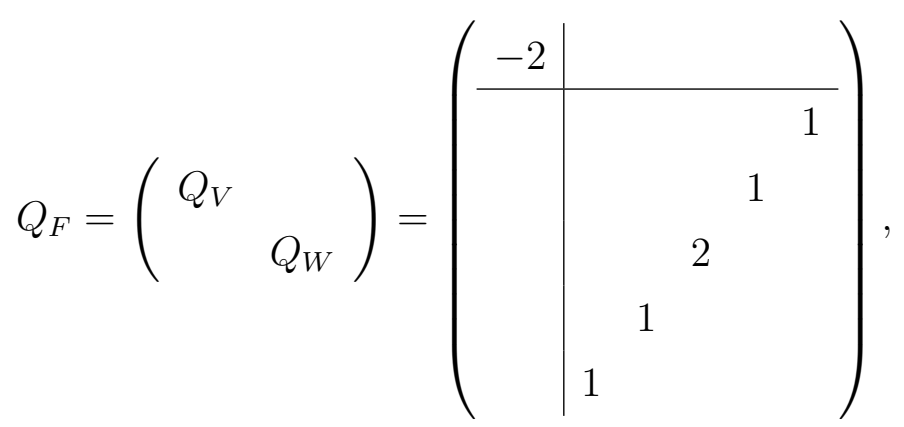

and the matrix of the Higgs field $\varphi$ of the corresponding $\mathrm{SO}_{0}(1,5)$-Higgs bundles is given by

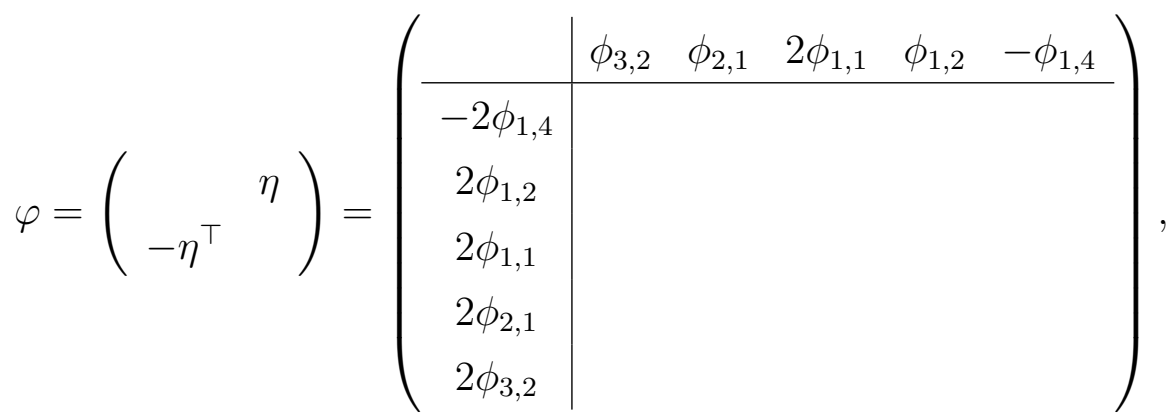

which satisfies $\varphi^{t} Q_{F}+Q_{F} \varphi=0$. We can establish the correspondence

$$
\begin{aligned}
\mathcal{M}\left(\mathrm{SU}^{*}(4)\right) & \rightarrow \mathcal{M}\left(\mathrm{SO}_{0}(1,5)\right) \\
(E, Q, \phi) & \mapsto\left(V, Q_{V}, W, Q_{W}, \eta\right) .
\end{aligned}
$$




\section{5 $\mathrm{SO}_{0}(2,4)$-Higgs bundles and $\mathrm{SO}^{*}(8)$-Higgs bundles}

The Lie algebra isomorphism $\mathfrak{s u}(4) \cong \mathfrak{s o}(6)$ together with Theorem 5.1 gives the following isomorphism

$$
\mathfrak{s o}^{*}(8) \cong \mathfrak{s o}(2,6)
$$

The isomorphism between the Lie groups is

$$
\mathrm{SO}^{*}(8) \cong \mathrm{SO}_{0}(2,4)
$$

Using this correspondence, we will establish a correspondence between the moduli space of polystable $\mathrm{SO}_{0}(2,6)$-Higgs bundles and the moduli space of polystable $\mathrm{SO}^{*}(8)$-Higgs bundles.

An $\mathrm{SO}^{*}(8)$-Higgs bundle is a triple $(E, \beta, \gamma)$, where $E$ is a holomorphic vector bundle of $\operatorname{rank} 4, \beta \in H^{0}\left(\Lambda^{2} E \otimes K\right)$ and $\gamma \in H^{0}\left(\Lambda^{2} E^{*} \otimes K\right)$. Let

$$
V=\Lambda^{4} E \oplus \Lambda^{4} E^{*} \quad \text { and } \quad W=\Lambda^{2} E \otimes \Lambda^{4} E^{*} .
$$

Since $\Lambda^{2} E$ has a non-degenerate quadratic form with values in $\Lambda^{4} E$ defined by

$$
\begin{aligned}
Q: \Lambda^{2} E \times \Lambda^{2} E & \rightarrow \Lambda^{4} E \\
\left(x \wedge y, x^{\prime} \wedge y^{\prime}\right) & \mapsto x \wedge y \wedge x^{\prime} \wedge y^{\prime},
\end{aligned}
$$

considering the induced quadratic form in $W$ we obtain an orthogonal bundle $\left(W, Q_{W}\right)$. To construct the Higgs field we observe that

$$
\begin{gathered}
\beta \in H^{0}\left(\Lambda^{2} E \otimes K\right) \cong H^{0}\left(\Lambda^{2} E \otimes \Lambda^{4} E^{*} \otimes \Lambda^{4} E \otimes K\right)=H^{0}\left(W \otimes \Lambda^{4} E \otimes K\right), \\
\gamma \in H^{0}\left(\Lambda^{2} E^{*} \otimes K\right) \cong H^{0}\left(\Lambda^{2} E^{*} \otimes \Lambda^{4} E \otimes \Lambda^{4} E^{*} \otimes K\right)=H^{0}\left(W^{*} \otimes \Lambda^{4} E^{*} \otimes K\right) .
\end{gathered}
$$

Since $W \cong W^{*}$, we can take

$$
\begin{aligned}
\eta: W & \rightarrow\left(\Lambda^{4} E \oplus \Lambda^{4} E^{*}\right) \otimes K \\
x & \mapsto(\beta(x), \gamma(x)) .
\end{aligned}
$$

and establish the correspondence

$$
\begin{aligned}
\mathcal{M}\left(\mathrm{SO}^{*}(8)\right) & \rightarrow \mathcal{M}\left(\mathrm{SO}_{0}(2,6)\right) \\
(E, \beta, \gamma) & \mapsto\left(V=\Lambda^{4} E \oplus \Lambda^{4} E^{*}, W=\Lambda^{2} E \otimes \Lambda^{4} E^{*}, Q_{W}, \eta\right)
\end{aligned}
$$




\section{Smooth minima}

We have seen in Section 4.4 that there is a homeomorphism between the moduli space of representations and the moduli space of Higgs bundles:

$$
\mathcal{R}_{c}\left(\mathrm{SO}_{0}(p, q)\right) \cong \mathcal{M}_{c}\left(\mathrm{SO}_{0}(p, q)\right)
$$

A first step in the study of the number of connected components of the space of representations $\mathcal{R}_{c}\left(\mathrm{SO}_{0}(p, q)\right)$ is to give a description of the minima of the Hitchin function in the moduli space $\mathcal{M}_{c}^{s}\left(\mathrm{SO}_{0}(p, q)\right)$ of smooth $\mathrm{SO}_{0}(p, q)$-Higgs bundles.

\subsection{The Hitchin function}

To simplify, we denote $\mathcal{M}=\mathcal{M}_{c}\left(\mathrm{SO}_{0}(p, q)\right)$.

Using the homeomorphism

$$
\mathcal{M}_{c}^{H i t}\left(\mathrm{SO}_{0}(p, q)\right) \cong \mathcal{M}
$$

of Section 4.3 , which identify $\mathcal{M}$ with the moduli space of solutions to Hitchin's equations

$$
\begin{aligned}
\mathcal{M}_{c}^{H i t}\left(\mathrm{SO}_{0}(p, q)\right)= & \{(A, \varphi) \mid A \text { connection on a smooth principal } \mathrm{SO}(p) \times \mathrm{SO}(q) \text {-bundle } \\
& \left.F, \varphi \in \Omega^{1,0}\left(F\left(\mathfrak{m}^{\mathbb{C}}\right)\right) \text { satisfying }(2) \text { and with } c(F)=c\right\} / \mathcal{G}(F),
\end{aligned}
$$

the Hitchin function is defined as the positive function

$$
f: \mathcal{M} \rightarrow \mathbb{R}
$$

given by

$$
[A, \varphi] \mapsto\|\varphi\|^{2}=\int_{X}|\varphi|^{2} d \mathrm{vol}
$$

where $[\cdot, \cdot]$ denotes the equivalence class in the moduli space $\mathcal{M}_{c}^{\text {Hit }}\left(\mathrm{SO}_{0}(p, q)\right)$ and $|\cdot|$ is the harmonic metric that gives the reduction to $\mathrm{SO}(p) \times \mathrm{SO}(q)$. Equivalently, we can define the map over the moduli space of Higgs pairs, for a fixed $(E, \varphi) \in \mathcal{M}$, by using the $L^{2}$-norm $\|\cdot\|$ of the metric that solves the Hitchin's equations.

Proposition 6.1. The function $f([A, \varphi])=\|\varphi\|^{2}$ is a proper map.

The proof is given by Hitchin in [31, Proposition 7.1].

Consider a compact subspace $[0, k] \subset \mathbb{R}$ and the inverse image

$$
f^{-1}[0, k]=\left\{[A, \varphi] \mid f([A, \varphi])=\|\varphi\|^{2} \leq k\right\} .
$$


A pair $[A, \varphi] \in f^{-1}[0, k]$ is a solution of the Hitchin's equations, that is,

$$
F_{A}-[\varphi, \tau(\varphi)]=0
$$

Since $\|[\varphi, \tau(\varphi)]\|^{2}$ is bounded by a multiple of $\|\varphi\|^{2}$, we have an $L^{2}$ bound of the curvature $F_{A}$, and using Uhlenbeck's weak compactness theorem, any infinite sequence in $f^{-1}[0, k]$ has convergent subsequence, and hence $f^{-1}[0, k]$ is compact.

Even if $\mathcal{M}$ is not smooth, as in our case, the fact that $f$ is a proper map gives information about the connected components of $\mathcal{M}$.

Proposition 6.2. Let $\mathcal{M}^{\prime} \subseteq \mathcal{M}$ be a closed subspace and let $\mathcal{N}^{\prime} \subseteq \mathcal{M}^{\prime}$ be the subspace of local minima of $f$ on $\mathcal{M}^{\prime}$. If $\mathcal{N}^{\prime}$ is connected, then $\mathcal{M}^{\prime}$ is connected.

This result is in fact more general. The proper function $f$ has a minimum on each connected component of $\mathcal{M}^{\prime}$, and then the number of connected components of $\mathcal{M}^{\prime}$ is bounded by the number of connected components of $\mathcal{N}^{\prime}$. Thus, we are interested in computing the critical points and more precisely the local minima of $f$.

We introduce now the notion of moment map for a hamiltonian action of a Lie group on a symplectic manifold to give more properties of the Hitchin function.

Let $(M, \omega)$ be a symplectic manifold, that is, a smooth manifold $M$ equipped with a closed non-degenerate 2 -form $\omega$ called the symplectic form. If we have a symplectic action of a Lie group $G$ on $M$,

$$
g^{*} \omega=\omega \text { for all } g \in G,
$$

a moment map for this action is a map

$$
\mu: M \rightarrow \mathfrak{g}^{*}
$$

such that

$$
d(\langle\mu, \xi\rangle)=i_{\tilde{\xi}} \omega, \text { for all } \xi \in \mathfrak{g},
$$

where $\tilde{\xi}$ is the vector field on $M$ induced by $\xi$, defined by

$$
\tilde{\xi}_{m} h=\lim _{t \rightarrow 0} \frac{h(\exp (t \xi) \cdot m)-h(m)}{t}
$$

for any smooth function $h: M \rightarrow \mathbb{R}$, and the map $\langle\mu, \xi\rangle: M \rightarrow \mathbb{R}$ is given by

$$
\langle\mu, \xi\rangle(m)=\langle\mu(m), \xi\rangle \text { for } m \in M,
$$


being $\langle\cdot, \cdot\rangle$ the pairing between $\mathfrak{g}$ and its dual. A vector field $v$ on $M$ is called Hamiltonian if

$$
i_{v} \omega=d f_{v}
$$

for a smooth function $f_{v}: M \rightarrow \mathbb{R}$. And the action of $G$ is said to be Hamiltonian if and only if the following two conditions hold. First, for every $\xi \in \mathfrak{g}$ the vector field $\tilde{\xi}$ is Hamiltonian, that is

$$
i_{\tilde{\xi}} \omega=d f_{\xi}
$$

for some smooth function $f_{\xi}: M \rightarrow \mathbb{R}$. Second, the map

$$
\xi \mapsto f_{\xi}
$$

is a Lie algebra homomorphism under the Poisson bracket

$$
\left\{f_{\xi}, f_{\xi^{\prime}}\right\}=\omega\left(\tilde{\xi}, \tilde{\xi}^{\prime}\right)
$$

If the action of $G$ on $(M, \omega)$ has a moment map $\mu$, for each $\xi \in \mathfrak{g}$ we can define

$$
f_{\xi}(m)=\langle\mu(m), \xi\rangle \text { for } m \in M,
$$

and then the action is Hamiltonian.

Proposition 6.3. The restriction of $f([A, \varphi])=\|\varphi\|^{2}$ to the smooth locus $\mathcal{M}^{s} \in \mathcal{M}$ is a moment map for the Hamiltonian circle action

$$
[A, \varphi] \mapsto\left[A, e^{i \theta} \varphi\right]
$$

We give a sketch of the proof of this result.

If $\mathcal{A}$ denotes the set of all connections on a principal $\mathrm{SO}(p) \times \mathrm{SO}(q)$-bundle $F$ and $\Omega=\Omega^{1,0}\left(F\left(\mathfrak{m}^{\mathbb{C}}\right)\right), \mathcal{A} \times \Omega$ is a symplectic manifold (see [31]). The action of the gauge group $\mathcal{G}(F)$ on $\mathcal{A} \times \Omega$ is symplectic, and the moment map of this action is

$$
\begin{aligned}
\mu: \mathcal{A} \times \Omega & \rightarrow(\text { Lie } \mathcal{G}(F))^{*} \\
(A, \varphi) & \mapsto F_{A}-[\varphi, \tau(\varphi)] .
\end{aligned}
$$

The subspace

$$
\mathcal{S}=\left\{(A, \varphi) \in \mathcal{A} \times \Omega \mid \bar{\partial}_{A}(\varphi)=0\right\}
$$

is invariant under the action of the gauge group and we can consider the restriction

$$
\mu_{0}: \mathcal{S} \rightarrow(\operatorname{Lie} \mathcal{G}(F))^{*}
$$


Using the isomorphism $\mathcal{M}_{c}^{\text {Hit }}\left(\mathrm{SO}_{0}(p, q)\right) \cong \mathcal{M}$, we have that $\mathcal{M}$ is isomorphic to the following symplectic quotient

$$
\mathcal{M} \cong \mu_{0}^{-1}(0) / \mathcal{G}(F)
$$

and then, the smooth moduli space $\mathcal{M}^{s}$ has structure of symplectic manifold.

The Lie group $\mathrm{U}(1)$, which is isomorphic to the sphere $S^{1}$, is a subgroup of the gauge group $\mathcal{G}(F)$ and then acts on $\mathcal{A} \times \Omega$ and on $\mathcal{S}$. But, since

$$
e^{i \theta} \cdot \varphi=e^{i \theta} \varphi e^{-i \theta}=\varphi
$$

this action is trivial on $\Omega$. Instead of this, from now on, we are going to consider the following circle action on $\mathcal{A} \times \Omega$ :

$$
e^{i \theta} \cdot(A, \varphi)=\left(A, e^{i \theta} \varphi\right)
$$

that can be defined as well on the moduli space of Higgs bundles $\mathcal{M}^{s}$,

$$
e^{i \theta} \cdot[A, \varphi]=\left[A, e^{i \theta} \varphi\right]
$$

Since the actions of $\mathrm{U}(1)$ and $\mathcal{G}(F)$ on $\mathcal{S}$ commute

$$
(\mathrm{U}(1) \times \mathcal{G}(F)) \times \mathcal{S} \rightarrow \mathcal{S}
$$

instead of consider the action of $\mathrm{U}(1)$ on $\mathcal{M}^{s}$ and find a moment map for this action, we are going to consider the action of $\mathrm{U}(1)$ on $\mathcal{S}$, to define the moment map for this action and after that, to translate our results to the moduli space $\mathcal{M}^{s}$.

The Lie algebra

$$
\mathfrak{u}(1)=\{z \in \mathbb{C} \mid z+\bar{z}=0\}
$$

is naturally identified with $i \mathbb{R}$ and using the isomorphism $\mathfrak{u}(1) \cong \mathfrak{u}(1)^{*}$ given by the Killing form, the moment map for this circle action on $\mathcal{S}$ is

$$
\begin{aligned}
\mu_{\mathrm{U}(1)}: \mathcal{S} & \rightarrow i \mathbb{R} \\
(A, \varphi) & \mapsto i\|\varphi\|^{2}=i \int_{X}|\varphi|^{2} d \mathrm{vol}
\end{aligned}
$$

The subspace $\mu_{0}^{-1}(0) \subset \mathcal{S}$ is invariant under the action of $\mathrm{U}(1)$, that is,

$$
F_{A}-\left[e^{i \theta} \varphi, \tau\left(e^{i \theta} \varphi\right)\right]=0
$$

for all $(A, \varphi) \in \mu_{0}^{-1}(0)$. Thus, the moment map for the action of $\mathrm{U}(1)$ on $\mu_{0}^{-1}(0)$ is the restriction

$$
\mu_{\mathrm{U}(1)}: \mu_{0}^{-1}(0) \rightarrow i \mathbb{R}
$$


Finally, since

$$
\|g \cdot \varphi\|^{2}=\int_{X}\left|g \varphi g^{-1}\right|^{2} d \mathrm{vol}=\|\varphi\|^{2}
$$

for all $g \in \mathcal{G}(F)$, this map is invariant under the action of $\mathcal{G}(F)$ and then we can restrict to the quotient $\mathcal{M} \cong \mu_{0}^{-1}(0) / \mathcal{G}(F)$ and finally to the smooth locus

$$
\mathcal{M}^{s} \rightarrow i \mathbb{R}
$$

If we consider $\mathfrak{u}(1)$ isomorphic to $\mathbb{R}$, the moment map takes values in $\mathbb{R}$.

\subsection{Morse Theory}

In this section we describe some properties of the Hitchin function which are related to Morse theory. Observe that these techniques can only be use when the moduli space $\mathcal{M}\left(\mathrm{SO}_{0}(p, q)\right)$ is smooth, and this only happens in a few cases, for small values of $p$ and $q$.

Let $f: \mathcal{M} \rightarrow \mathbb{R}$ be a smooth function on a manifold $\mathcal{M}$. A connected submanifold $\mathcal{M}^{\prime} \subset \mathcal{M}$ is a critical manifold for $f$ if and only if $d f \equiv 0$ along $\mathcal{M}^{\prime}$, and we say that $\mathcal{M}^{\prime}$ is non-degenerate if the Hessian of $f$ is non-degenerate on the normal bundle to $\mathcal{M}^{\prime}$. Let $\mathcal{M}_{l}$ be the critical manifolds of $\mathcal{M}$ and denote the manifold of local minima of $f$ by $\mathcal{N}=\mathcal{M}_{0}$.

The function $f: \mathcal{M} \rightarrow \mathbb{R}$ is a Bott-Morse function if all the critical manifolds $\mathcal{M}_{l}$ are non-degenerated.

Let $\nu\left(\mathcal{M}_{l}\right)$ be the normal bundle of the critical manifold $\mathcal{M}_{l}$. The non-degeneracy of the Hessian implies, for any critical manifold, the decomposition

$$
\nu\left(\mathcal{M}_{l}\right)=\nu^{+}\left(\mathcal{M}_{l}\right) \oplus \nu^{-}\left(\mathcal{M}_{l}\right)
$$

where $\nu^{+}\left(\mathcal{M}_{l}\right)$ and $\nu^{-}\left(\mathcal{M}_{l}\right)$ are the subbundles where the Hessian is positive and negative defined respectively.

The real dimension of $\nu^{-}\left(\mathcal{M}_{l}\right)$ is called the Morse index of $\mathcal{M}_{l}$, denoted by

$$
\lambda_{l}=\operatorname{index}\left(\mathcal{M}_{l}\right)=\operatorname{rk} \nu^{-}\left(\mathcal{M}_{l}\right) .
$$

Define the Poincaré polynomial of $\mathcal{M}$ to be

$$
P_{t}(\mathcal{M})=\sum t^{i} \operatorname{dim} H^{i}(\mathcal{M}, \mathbb{Q}) .
$$

For the critical manifolds we have the same definition

$$
P_{t}\left(\mathcal{M}_{l}\right)=\sum t^{i} \operatorname{dim} H^{i}\left(\mathcal{M}_{l}, \mathbb{Q}\right)
$$


The coefficient of $t^{i}$ is called the $i$-th Betti number of $\mathcal{M}$. These numbers are topological invariants of $\mathcal{M}$.

A Bott-Morse function is called perfect if

$$
P_{t}(\mathcal{M})=\sum_{l} t^{\lambda_{l}} P_{t}\left(\mathcal{M}_{l}\right)
$$

Proposition 6.4. If $\mathcal{M}=\mathcal{M}\left(\mathrm{SO}_{0}(p, q)\right)$ is smooth, then the Hitchin function $f$ is a perfect Bott-Morse function.

\subsection{Critical points of the Hitchin function}

To study the critical points of the Hitchin function we use the following result.

Proposition 6.5. A smooth point of the moduli space $\mathcal{M}$ is a critical point of $f$ if and only if it is a fixed point of the circle action, and the subbundle $\nu^{-}\left(\mathcal{M}_{l}\right)$ where the Hessian of the Hitchin function is negative definite equals the subbundle of $\nu\left(\mathcal{M}_{l}\right)$ on which the circle acts with negative weights.

Proof. The moduli space $\mathcal{M}^{s}$ is a Kähler manifold, that is, it is a complex manifold $(\mathcal{M}, I)$, which also carries a Riemannian metric $g$ and a symplectic form $\omega$ on the underlying real manifold in such a way that the three structures (complex, Riemannian, and symplectic) are all mutually compatible,

$$
\omega(\cdot, \cdot)=g(I \cdot, \cdot) \text { on } T \mathcal{M}^{s} \times T \mathcal{M}^{s}
$$

The metric $g$ induces the isomorphism

$$
\begin{aligned}
T \mathcal{M}^{s} & \rightarrow T^{*} \mathcal{M}^{s} \\
v & \mapsto g(v, \cdot) .
\end{aligned}
$$

If $f: \mathcal{M}^{s} \rightarrow \mathbb{R}$ is a smooth map, the element of $T \mathcal{M}^{s}$ corresponding to $d f \in T^{*} \mathcal{M}^{s}$ is $\operatorname{grad}(f)$. Applying this to the Hitchin function, we have

$$
d f=g(\operatorname{grad}(f), \cdot)
$$

Proposition 6.3 tells us that $f$, restricted to the smooth locus, is a moment map for the circle action. In this case, since $\mathfrak{u}(1) \cong \mathbb{R}$, the moment map $f$ is the Hamiltonian function that generates the circle action and

$$
d f=\omega(u, \cdot)=g(I u, \cdot)
$$


where $I$ is the complex structure of $\mathcal{M}^{s}$ and $u$ is the vector field generating the circle action, that is

$$
u_{[A, \varphi]} h=\lim _{\theta \rightarrow 0} \frac{h\left(\left[A, e^{i \theta} \varphi\right]\right)-h([A, \varphi])}{\theta}
$$

for any $h: \mathcal{M}^{s} \rightarrow \mathbb{R}$. Thus,

$$
\operatorname{grad}(f)=I u \text {. }
$$

Then, the critical points of $f$ are the smooth points of $\mathcal{M}$ where the gradient vanishes and $u$ is null on the fixed points of the action.

If $m=[A, \varphi]$ is a fixed point of the circle action, the infinitesimal circle action on $T_{m} \mathcal{M}^{s}$ is given by

$$
v_{m} \mapsto[v, u]_{m} .
$$

On the other hand, if $\nabla$ is the Levi-Civita connection on $\mathcal{M}^{s}$ and $H_{f}$ denotes the Hessian of $f$, we have

$$
H_{f}\left(v_{m}\right)=\nabla_{v_{m}}(\operatorname{grad}(f))=\nabla_{v_{m}}(I u)=\nabla_{I u_{m}}(v)-[I u, v]_{m}=[v, I u]_{m},
$$

where we have used that $u_{m}=0$.

Using Proposition 6.5, the critical points of $f$ are of two types:

(1) The Higgs field $\varphi=0$.

(2) If $\varphi \neq 0,[A, \varphi]$ is a fixed point of the circle action if and only if

$$
\left[A, e^{i \theta} \varphi\right]=[A, \varphi], \text { for all } e^{i \theta} \in S^{1}
$$

Then, there is a 1-parameter family of gauge transformations $g(\theta)=\left(g_{1}(\theta), g_{2}(\theta)\right) \in \mathcal{G}(F)$ such that

$$
\left(A, e^{i \theta} \varphi\right)=g(\theta) \cdot(A, \varphi)=(g(\theta) \cdot A, g(\theta) \cdot \varphi)
$$

We have that

$$
g(\theta) \cdot \varphi=\iota(g(\theta))(\varphi)=\operatorname{Ad}(g(\theta))(\varphi)=\exp (\operatorname{ad}(\theta \psi))(\varphi)
$$

and taking $\left.\frac{d}{d \theta}\right|_{\theta=0}$ in the second term of the parenthesis in (3) we obtain

$$
\left.\frac{d}{d \theta}\left(e^{i \theta} \varphi\right)\right|_{\theta=0}=i \varphi
$$

and

$$
\left.\frac{d}{d \theta}(g(\theta) \cdot \varphi)\right|_{\theta=0}=\left.\frac{d}{d \theta} \exp (\operatorname{ad}(\theta \psi))(\varphi)\right|_{\theta=0}=\operatorname{ad}(\psi)(\varphi)=[\psi, \varphi]
$$


Then

$$
[\psi, \varphi]=i \varphi
$$

Let $A=\left(A_{1}, A_{2}\right)$. Since $g_{1}(\theta)$ and $g_{2}(\theta)$ act on $A_{1}$ and $A_{2}$ separately, we can consider $\psi_{1}$ and $\psi_{2}$ generating the action of $\left\{g_{1}(\theta)\right\}$ and $\left\{g_{2}(\theta)\right\}$. The equation (3) gives the following condition for the action on the connections

$$
g_{i}(\theta) \cdot A_{i}=g_{i}(\theta) \circ A_{i} \circ g_{i}(\theta)^{-1}=A_{i},
$$

or equivalently

$$
A_{i} \circ g_{i}(\theta)=g_{i}(\theta) \circ A_{i},
$$

that is, the automorphism $g_{i}(\theta)$ is parallel with respect to the connection $A_{i}$. Then we have

$$
d_{A_{i}}\left(\psi_{i}\right)=0
$$

That is, the family $\left\{g(\theta)=\left(g_{1}(\theta), g_{2}(\theta)\right)\right\}$ is generated by an infinitesimal gauge transformation $\psi=\left(\psi_{1}, \psi_{2}\right)$ which is covariantly constant, that is,

$$
d_{A_{1}}\left(\psi_{1}\right)=d_{A_{2}}\left(\psi_{2}\right)=0
$$

and with

$$
[\psi, \varphi]=i \varphi
$$

Proposition 6.6. An $\mathrm{SO}_{0}(p, q)$-Higgs bundle $\left(V, Q_{V}, W, Q_{W}, \eta\right) \in \mathcal{M}$ represents a fixed point of the circle action if and only it is a Hodge bundle (complex variation of Hodge structure), that is, if and only if the vector bundles $V$ and $W$ have decompositions

$$
V=\bigoplus_{m=-r}^{r} V_{m} \text { and } W=\bigoplus_{n=-s}^{s} W_{n},
$$

with $V_{m} \cong\left(V^{*}\right)_{-m}, W_{n} \cong\left(W^{*}\right)_{-n}$ and $\left.\psi_{1}\right|_{V_{m}}=i m$ and $\left.\psi_{2}\right|_{W_{n}}=$ in for an infinitesimal gauge transformation $\psi=\left(\psi_{1}, \psi_{2}\right)$, with

$$
\eta_{k}: W_{k} \rightarrow V_{k+1} \otimes K
$$

Proof. If $\left(V, Q_{V}, W, Q_{W}, \eta\right)$ represents a smooth point of the moduli space which is a critical point of $f$, as we have seen in the previous section, there is an endomorphism $\psi=\left(\psi_{1}, \psi_{2}\right)$ that gives decompositions

$$
V=\bigoplus_{m} V_{m} \text { and } W=\bigoplus_{n} W_{n}
$$


where $\underline{m, n \in \mathbb{R}},\left.\psi_{1}\right|_{V_{m}}=i m$ and $\left.\psi_{2}\right|_{W_{n}}=i n$. Moreover, since $\psi_{1}$ and $\psi_{2}$ are locally in $\mathfrak{s o}(p)$ and $\mathfrak{s o}(q)$ respectively, they satisfy $\psi_{1}=-\psi_{1}^{\top}$ and $\psi_{2}=-\psi_{2}^{\top}$. If $q_{V}: V \cong V^{*}$ is the isomorphism given by the orthogonal form $Q_{V}$, we have $\psi_{1}^{\top}=q_{V}^{-1} \circ \psi_{1}^{t} \circ q_{V}$, and for all $v \in V_{m}$ we have

$$
\psi_{1}^{t}\left(q_{V}(v)\right)=q_{V}\left(\psi_{1}^{\top}(v)\right)=-q_{V}\left(\psi_{1}(v)\right)=-i m q_{V}(v)
$$

that is,

$$
v \in V_{m} \Leftrightarrow q_{V}(v) \in\left(V^{*}\right)_{-m}
$$

Hence, we have an isomorphism $V_{m} \cong\left(V^{*}\right)_{-m}$. The same argument applied to $\psi_{2}, q_{W}$ and $W$ gives an isomorphism $W_{n} \cong\left(W^{*}\right)_{-n}$.

If $v \in V_{m}$ and $v^{\prime} \in V_{l}$,

$$
Q_{V}\left(\psi_{1}(v), v^{\prime}\right)=Q_{V}\left(i m v, v^{\prime}\right)=i m Q_{V}\left(v, v^{\prime}\right)
$$

and, on the other hand,

$$
Q_{V}\left(\psi_{1}(v), v^{\prime}\right)=Q_{V}\left(v, \psi_{1}^{\top}\left(v^{\prime}\right)\right)=Q_{V}\left(v,-\psi_{1}\left(v^{\prime}\right)\right)=Q_{V}\left(v,-i l v^{\prime}\right)=-i l Q_{V}\left(v, v^{\prime}\right),
$$

that is,

$$
i(m+l) Q_{V}\left(v, v^{\prime}\right)=0 .
$$

Then, all the $V_{l}$ are orthogonal to $V_{m}$ (including $l=m$ ) under $Q_{V}$ except $l=-m$. Since $Q_{V}$ is non-degenerate,

$$
Q_{V}\left(v, v^{\prime}\right)=0 \text { for all } v^{\prime} \in V \Rightarrow v=0
$$

and then, given $0 \neq v \in V_{m}$, there is a $v^{\prime} \in V$ with $Q_{V}\left(v, v^{\prime}\right) \neq 0$, that is, a $v^{\prime} \in V_{-m}$. The same for $W, Q_{W}$ and $\psi_{2}$. Then

$$
V=\bigoplus_{m=-r}^{r} V_{m} \text { and } W=\bigoplus_{n=-s}^{s} W_{n}
$$

We also know that the endomorphisms $\psi_{1}$ and $\psi_{2}$ are trace free, then

$$
\begin{gathered}
0=\operatorname{Tr}\left(\psi_{1}\right)=i \sum_{m=-r}^{r} m \operatorname{rk}\left(V_{m}\right) \Leftrightarrow \sum_{m=-r}^{r} m \operatorname{rk}\left(V_{m}\right)=0 \\
0=\operatorname{Tr}\left(\psi_{2}\right)=i \sum_{n=-s}^{s} n \operatorname{rk}\left(W_{n}\right) \Leftrightarrow \sum_{n=-s}^{s} n \operatorname{rk}\left(W_{n}\right)=0 .
\end{gathered}
$$

The condition $[\psi, \varphi]=i \varphi$ for the solution $(A, \varphi)$ is equivalent in this context to

$$
\psi_{1} \eta-\eta \psi_{2}=i \eta
$$


If $w \in W_{k}$, we have

$$
\psi_{1}(\eta(w))=\eta\left(\psi_{2}(w)\right)+i \eta(w)=\eta(i k w)+i \eta(w)=i(k+1) \eta(w),
$$

that is,

$$
\eta_{k}: W_{k} \rightarrow V_{k+1} \otimes K
$$

and we conclude.

If $\left(V, Q_{V}, W, Q_{W}, \eta\right)$ is a Hodge bundle and

$$
\left(\mathbb{E}=V \oplus W, Q=\left(\begin{array}{ll}
Q_{V} & \\
& Q_{W}
\end{array}\right), \phi=\left(\begin{array}{cc}
0 & \eta \\
-\eta^{\top} & 0
\end{array}\right)\right)
$$

is the $\mathrm{SO}(p+q, \mathbb{C})$-Higgs bundle associated to it as in Section 3.4 the decompositions of $V$ and $W$ give a decomposition

$$
\mathbb{E}=V \oplus W=\bigoplus_{k} \mathbb{E}_{k}=\bigoplus_{k}\left(V_{k} \oplus W_{k}\right),
$$

where $\mathbb{E}_{k}$ is the eigenbundle of eigenvalue $i k$ for the action of the endomorphism $\psi=$ $\left(\begin{array}{cc}\psi_{1} & 0 \\ 0 & \psi_{2}\end{array}\right) \in \operatorname{End}(\mathbb{E})$, that is, $V_{k}$ and $W_{k}$ are eigenbundles for $\psi_{1}$ and $\psi_{2}$ respectively with eigenvalue $i k$. The quadratic form $Q$ gives isomorphisms $\mathbb{E}_{l} \cong \mathbb{E}_{-l}^{*}$ and the remaining $\mathbb{E}_{k}$ are orthogonal to $\mathbb{E}_{l}$ under $Q$. Then, the decomposition of $\mathbb{E}$ is of the form

$$
\mathbb{E}=V \oplus W=\bigoplus_{k=-l}^{l} \mathbb{E}_{k}=\bigoplus_{k=-l}^{l}\left(V_{k} \oplus W_{k}\right)
$$

where $l=\max \{r, s\}$.

The restriction of the Higgs field to each $\mathbb{E}_{k}$ satisfies

$$
\varphi_{k}=\left(\begin{array}{cc}
0 & \eta_{k} \\
\left(-\eta^{\top}\right)_{k} & 0
\end{array}\right): \mathbb{E}_{k} \rightarrow \mathbb{E}_{k+1} \otimes K
$$

where

$$
\eta_{k}: W_{k} \rightarrow V_{k+1} \otimes K \text { and }\left(-\eta^{\top}\right)_{k}: V_{k} \rightarrow W_{k+1} \otimes K .
$$

Moreover, since the orthogonal structures on $V$ and $W$ give the following commutative diagram

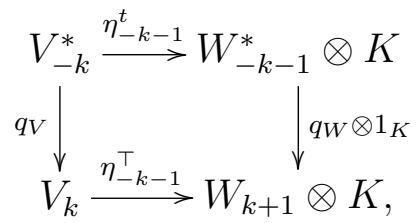


we have that

$$
\left(-\eta^{\top}\right)_{k}=-\eta_{-k-1}^{\top}
$$

Analogously, if $q: \mathbb{E} \cong \mathbb{E}^{*}$ is the isomorphism given by the orthogonal structure $Q$ of $\mathbb{E}$, the commutative diagram

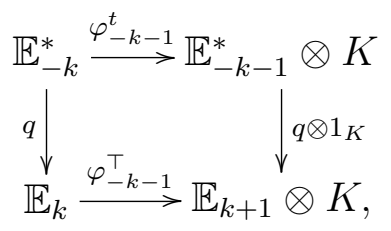

tells us that

$$
\varphi_{k} \cong \varphi_{-k-1}^{\top}
$$

The general picture is the following

$$
\begin{gathered}
\mathbb{E}_{-m} \stackrel{\varphi_{-m}}{\longrightarrow} \mathbb{E}_{-m+1} \stackrel{\varphi_{-m+1}}{\longrightarrow} \mathbb{E}_{-m+2} \stackrel{\varphi_{-m+2}}{\longrightarrow} \cdots \stackrel{\varphi_{m-3}}{\longrightarrow} \mathbb{E}_{m-2} \stackrel{\varphi_{m-2}}{\longrightarrow} \mathbb{E}_{m-1} \stackrel{\varphi_{m-1}}{\longrightarrow} \mathbb{E}_{m} \\
V_{-m} \stackrel{-\eta_{m-1}^{\top}}{\longrightarrow} W_{-m+1} \stackrel{\eta_{-m+1}}{\longrightarrow} V_{-m+2} \stackrel{-\eta_{m-3}^{\top}}{\longrightarrow} \cdots \stackrel{\eta_{m-3}}{\longrightarrow} V_{m-2} \stackrel{-\eta_{-m+1}^{\top}}{\longrightarrow} W_{m-1} \stackrel{\eta_{m-1}}{\longrightarrow} V_{m} \\
\oplus \quad \cdots \quad \oplus \\
W_{-m} \stackrel{\eta_{-m}}{\longrightarrow} V_{-m+1} \stackrel{-\eta_{m-2}^{\top}}{\longrightarrow} W_{-m+2} \stackrel{\eta_{-m+2}}{\longrightarrow} \cdots \stackrel{-\eta_{-m+2}^{\top}}{\longrightarrow} W_{m-2} \stackrel{\eta_{m-2}}{\longrightarrow} V_{m-1} \stackrel{-\eta_{-m}^{\top}}{\longrightarrow} W_{m} .
\end{gathered}
$$

Note that the arrows represents the Higgs field and are twisted by $K$.

Theorem 6.6 together with Proposition 6.5 tell us that if $\left(V, Q_{V}, W, Q_{W}, \eta\right)$ is an $\mathrm{SO}_{0}(p, q)$-Higgs bundle which represents a smooth point of the moduli space, it is a critical point of the Hitchin function if and only if it is a Hodge bundle, but observe that not every Hodge bundle represents a smooth point.

\subsection{Criterion for minima}

Let $(E, \varphi)$ be an $\mathrm{SO}_{0}(p, q)$-Higgs bundle and let $\left(E_{\mathrm{SO}(p+q, \mathbb{C})}, \varphi\right)$ be the associated $\mathrm{SO}(p+$ $q, \mathbb{C})$-Higgs bundle. Consider also the tuple $\left(V, Q_{V}, W, Q_{W}, \eta\right)$ corresponding to $(E, \varphi)$ 
and the triple $(\mathbb{E}, Q, \varphi)$ corresponding to $\left(E_{\mathrm{SO}(p+q, \mathbb{C})}, \varphi\right)$. We have that

$$
\begin{aligned}
E_{\mathrm{SO}(p+q, \mathbb{C})}(\mathfrak{s o}(p+q, \mathbb{C}))= & \left\{f \in \operatorname{End}(\mathbb{E}) \mid f+f^{\top}=0\right\}=\mathfrak{s o}(\mathbb{E}), \\
E\left(\mathfrak{h}^{\mathbb{C}}\right)= & \left\{\left(\begin{array}{cc}
f_{1} & 0 \\
0 & f_{4}
\end{array}\right) \in \operatorname{End}(\mathbb{E}) \mid f_{1}+f_{1}^{\top}=0 \text { and } f_{4}+f_{4}^{\top}=0\right\} \\
& \cong \mathfrak{s o}(V) \oplus \mathfrak{s o}(W) \subset \operatorname{End}(V) \oplus \operatorname{End}(W), \\
E\left(\mathfrak{m}^{\mathbb{C}}\right)= & \left\{\left(\begin{array}{cc}
0 & f_{2} \\
-f_{2}^{\top} & 0
\end{array}\right) \in \operatorname{End}(\mathbb{E})\right\} \cong \operatorname{Hom}(W, V) .
\end{aligned}
$$

In fact,

$$
E_{\mathrm{SO}(p+q, \mathbb{C})}(\mathfrak{s o}(p+q, \mathbb{C}))=E\left(\mathfrak{h}^{\mathbb{C}}\right) \oplus E\left(\mathfrak{m}^{\mathbb{C}}\right),
$$

which is induced by the Cartan decomposition of the Lie algebra $\mathfrak{s} \mathfrak{o}(p+q, \mathbb{C})$.

If $\left(V, Q_{V}, W, Q_{W}, \eta\right)$ is a Hodge bundle, Proposition 6.6 tells us that there is an infinitesimal gauge transformation $\psi=\left(\psi_{1}, \psi_{2}\right)$ such that

$$
V=\bigoplus_{m=-r}^{r} V_{m} \text { and } W=\bigoplus_{n=-s}^{s} W_{n}
$$

with $V_{m} \cong\left(V^{*}\right)_{-m}, W_{n} \cong\left(W^{*}\right)_{-n},\left.\psi_{1}\right|_{V_{m}}=i m,\left.\psi_{2}\right|_{W_{n}}=i n$ and

$$
\eta_{k}: W_{k} \rightarrow V_{k+1} \otimes K
$$

This decompositions of $V$ and $W$ give decompositions

$$
\begin{aligned}
\operatorname{End}(V)_{k} & =\bigoplus_{k=-2 r}^{2 r}\left(\bigoplus_{i-j=k} \operatorname{Hom}\left(V_{j}, V_{i}\right)\right), \\
\operatorname{End}(W)_{k} & =\bigoplus_{k=-2 s}^{2 s}\left(\bigoplus_{i-j=k} \operatorname{Hom}\left(W_{j}, W_{i}\right)\right), \\
\operatorname{Hom}(W, V)_{k} & =\bigoplus_{k=-r-s}^{r+s}\left(\bigoplus_{i-j=k} \operatorname{Hom}\left(W_{j}, V_{i}\right)\right), \\
\operatorname{Hom}(V, W)_{k} & =\bigoplus_{k=-r-s}^{r+s}\left(\bigoplus_{i-j=k} \operatorname{Hom}\left(V_{j}, W_{i}\right)\right) .
\end{aligned}
$$

If $f_{k, l} \in \operatorname{Hom}\left(V_{k}, V_{l}\right)$ and $g_{k, l} \in \operatorname{Hom}\left(W_{k}, W_{l}\right)$, using the isomorphisms $q_{V}$ and $q_{W}$ induced by the orthogonal forms $Q_{V}$ and $Q_{W}$ we have that the diagrams

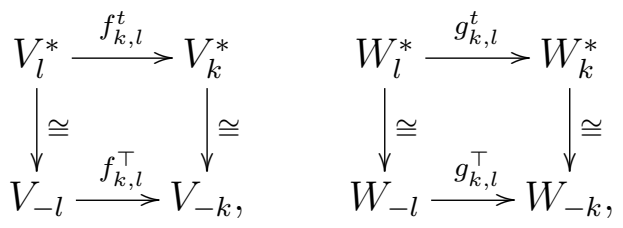


are commutative, and then, the skew-symmetry in $E\left(\mathfrak{h}^{\mathbb{C}}\right) \cong \mathfrak{s o}(V) \oplus \mathfrak{s o}(W) \subset \operatorname{End}(V) \oplus$ $\operatorname{End}(W)$ is equivalent to the conditions:

$$
\begin{aligned}
& f_{-l,-k}+f_{k, l}^{\top}=0, \\
& g_{-l,-k}+g_{k, l}^{\top}=0,
\end{aligned}
$$

that is, the following sets are related by skew-symmetry

$$
\begin{aligned}
& f_{k, l} \longleftrightarrow-f_{k, l}^{\top}, \quad g_{k, l} \longleftrightarrow-g_{k, l}^{\top}, \\
& \operatorname{Hom}\left(V_{k}, V_{l}\right) \longleftrightarrow \operatorname{Hom}\left(V_{-l}, V_{-k}\right), \quad \operatorname{Hom}\left(W_{k}, W_{l}\right) \longleftrightarrow \operatorname{Hom}\left(W_{-l}, W_{-k}\right) .
\end{aligned}
$$

Observe that when $k=l$, the endomorphism $f_{h, l}$ and $g_{k, l}$ are skew-symmetric. Analogously, in $E\left(\mathfrak{m}^{\mathbb{C}}\right)$ we have the relation:

$$
\begin{aligned}
h_{k, l} & \longleftrightarrow-h_{k, l}^{\top}, \\
\operatorname{Hom}\left(W_{k}, V_{l}\right) & \longleftrightarrow \operatorname{Hom}\left(V_{-l}, W_{-k}\right) .
\end{aligned}
$$

Then, the decompositions of $V$ and $W$ also induce decompositions of $E\left(\mathfrak{h}^{\mathbb{C}}\right) \cong \mathfrak{s o}(V) \oplus$ $\mathfrak{s o}(W)$ and $E\left(\mathfrak{m}^{\mathbb{C}}\right) \cong \operatorname{Hom}(W, V)$, and this gives a decomposition of the deformation complex of Section 3.5 .

$$
C^{\bullet}(E, \varphi): 0 \longrightarrow E\left(\mathfrak{h}^{\mathbb{C}}\right) \stackrel{\operatorname{ad}(\varphi)}{\longrightarrow} E\left(\mathfrak{m}^{\mathbb{C}}\right) \otimes K \longrightarrow 0
$$

that is,

$$
C^{\bullet}(E, \varphi)=\bigoplus_{k} C_{k}^{\bullet}(E, \varphi)
$$

where $C_{k}^{\bullet}(E, \varphi)$ are the subcomplexes

$$
C_{k}^{\bullet}(E, \varphi): 0 \longrightarrow E\left(\mathfrak{h}^{\mathbb{C}}\right)_{k} \stackrel{\operatorname{ad}(\varphi)}{\longrightarrow} E\left(\mathfrak{h}^{\mathbb{C}}\right)_{k+1} \otimes K \longrightarrow 0,
$$

where the action of $\operatorname{ad}(\varphi)$ takes $E\left(\mathfrak{h}^{\mathbb{C}}\right)_{k}$ into $E\left(\mathfrak{m}^{\mathbb{C}}\right)_{k+1}$ since

$$
[\psi,[\varphi, f]]=[[\psi, \varphi], f]+[\varphi,[\psi, f]]=[i \varphi, f]+[\varphi, i k f]=i(k+1)[\varphi, f],
$$

for all $f \in E\left(\mathfrak{h}^{\mathbb{C}}\right)_{k}$. This induces a decomposition of the infinitesimal deformation space given by

$$
\mathbb{H}^{1}\left(C^{\bullet}(E, \varphi)\right)=\bigoplus_{k} \mathbb{H}^{1}\left(C_{k}^{\bullet}(E, \varphi)\right)
$$

Proposition 6.7. Let $(E, \varphi)$ be an $\mathrm{SO}_{0}(p, q)$-Higgs bundle which represents a smooth point of the moduli space $\mathcal{M}$ and which is a critical point of $f$. The hypercohomology group $\mathbb{H}^{1}\left(C_{k}^{\bullet}(E, \varphi)\right)$ is isomorphic to the eigenspace of the Hessian of $f$ with eigenvalue $-k$. Then, the point $(E, \varphi)$ corresponds to a local minimum of $f$ if and only if

$$
\mathbb{H}^{1}\left(C_{k}^{\bullet}(E, \varphi)\right)=0 \text { for } k>0 .
$$


If $[E, \varphi] \in \mathcal{M}^{s}$ ( $s$ for smooth) is a critical point of $f$, then $[E, \varphi] \in \mathcal{M}_{l}$ for some critical manifold $\mathcal{M}_{l}$ and we define the Morse index at the point $[E, \varphi]$ as the Morse index of $\mathcal{M}_{l}$. The first part of this proposition alow us to calculate the Morse index of $[E, \varphi]$, which is

$$
\text { index }=\sum_{k>0} \operatorname{dim}_{\mathbb{R}} \mathbb{H}^{1}\left(C_{k}^{\bullet}(E, \varphi)\right)
$$

If $[E, \varphi] \in \mathcal{M}^{s}$ is a minimum of $f$, that is $[E, \varphi] \in \mathcal{N}$, then

$$
\mathbb{H}^{1}\left(C_{k}^{\bullet}(E, \varphi)\right)=0 \text { for } k>0
$$

and the Morse index at $[E, \varphi]$ is null.

To give a criterion for deciding when the hypercohomology $\mathbb{H}\left(C_{k}^{\bullet}(E, \varphi)\right)$ vanishes, we use the Euler characteristic of the complex $C_{k}^{\bullet}(E, \varphi)$ defined by

$$
\chi\left(C_{k}^{\bullet}(E, \varphi)\right)=\operatorname{dim} \mathbb{H}^{0}\left(C_{k}^{\bullet}(E, \varphi)\right)-\operatorname{dim} \mathbb{H}^{1}\left(C_{k}^{\bullet}(E, \varphi)\right)+\operatorname{dim} \mathbb{H}^{2}\left(C_{k}^{\bullet}(E, \varphi)\right) .
$$

Proposition 6.8. Let $(E, \varphi)$ be an $\mathrm{SO}_{0}(p, q)$-Higgs bundle which represents a fixed point under the circle action on $\mathcal{M}$. Then

$$
\chi\left(C_{k}^{\bullet}(E, \varphi)\right) \leq 0
$$

and equality holds if and only if the map

$$
\operatorname{ad}(\varphi): E\left(\mathfrak{h}^{\mathbb{C}}\right)_{k} \rightarrow E\left(\mathfrak{m}^{\mathbb{C}}\right)_{k+1} \otimes K
$$

is an isomorphism.

If $(E, \varphi)$ represents a smooth $\mathrm{SO}_{0}(p, q)$-Higgs bundle, using the Corollary 3.16, we have that

$$
\mathbb{H}^{0}\left(C_{k}^{\bullet}(E, \varphi)\right)=\mathbb{H}^{2}\left(C_{k}^{\bullet}(E, \varphi)\right)=0
$$

and then,

$$
-\chi\left(C_{k}^{\bullet}(E, \varphi)\right)=\operatorname{dim} \mathbb{H}^{1}\left(C_{k}^{\bullet}(E, \varphi)\right),
$$

for all $k$. Applying Proposition 6.7, we have the following criterion for local minima of $f$.

Proposition 6.9. Let $(E, \varphi)$ be an $\mathrm{SO}_{0}(p, q)$-Higgs bundle which represents a smooth point of $\mathcal{M}$ and which is a critical point of $f$. Then it represents a local minimum if and only if

$$
\operatorname{ad}(\varphi): E\left(\mathfrak{h}^{\mathbb{C}}\right)_{k} \rightarrow E\left(\mathfrak{m}^{\mathbb{C}}\right)_{k+1} \otimes K
$$

is an isomorphism for all $k>0$. 
This criterion can be used only when the $\mathrm{SO}_{0}(p, q)$-Higgs bundle represents a smooth point of the moduli space. If $(E, \varphi)$ does not represent a smooth point, it is shown in [32] that if $\mathbb{H}^{1}\left(C_{k}^{\bullet}(E, \varphi)\right)=0$ for all $k>0$ then $(E, \varphi)$ is a local minimum, but we will see in Proposition 7.3 that to have

$$
\mathbb{H}^{1}\left(C_{-}^{\bullet}(E, \varphi)\right)=\bigoplus_{k>0} \mathbb{H}^{1}\left(C_{k}^{\bullet}(E, \varphi)\right) \neq 0
$$

is not enough to prove that the point is not a minimum.

\subsection{Smooth minima}

In this section we describe the minima of the Hitchin function in the smooth locus $\mathcal{M}^{s}$ using the criterion of Proposition 6.9. Since the Hitchin function is proper in $\mathcal{M}$ (see Proposition 6.1) but not in $\mathcal{M}^{s}$, to find these minima does not allow us to count the number of connected components of $\mathcal{M}^{s}$, but it is the first step in the study of the connected components of $\mathcal{M}$.

We will consider in all this section Higgs bundles with non-zero Higgs field.

Theorem 6.10. Let $\left(V, Q_{V}, W, Q_{W}, \eta\right)$ be an $\mathrm{SO}_{0}(p, q)$-Higgs bundle, with $p \leq q$, which represents a smooth point of the moduli space and which is a local minimum of the Hitchin function. If the Higgs field is not equal to zero, the minimum is in one of the following cases:

(1) $p \geq 2$ even, $q=p+1$ and

$$
W_{-p} \rightarrow V_{-p+1} \rightarrow \cdots \rightarrow V_{-1} \rightarrow W_{0} \rightarrow V_{1} \rightarrow \cdots \rightarrow V_{p-1} \rightarrow W_{p}
$$

with all subbundles of rank 1 ,

$$
V_{k} \text { or } W_{k} \cong K^{-k} \text { for } 0 \leq k \leq p-1
$$

and

$$
0<\operatorname{deg}\left(W_{-p}\right) \leq p(2 g-2)
$$

(2) $p \geq 1$ odd, $q=p+1$ and

$$
W_{-p} \rightarrow V_{-p+1} \rightarrow \cdots \rightarrow W_{-1} \rightarrow V_{0} \rightarrow W_{1} \rightarrow \cdots \rightarrow V_{p-1} \rightarrow W_{p}
$$

with all subbundles of rank 1 ,

$$
V_{k} \text { or } W_{k} \cong K^{-k} \text { for } 0 \leq k \leq p-1
$$


and

$$
0<\operatorname{deg}\left(W_{-p}\right) \leq p(2 g-2)
$$

(3) $p \geq 2$ even, $q=p-2+n$ with $n \geq 2$ and

$$
V_{-p+1} \rightarrow W_{-p+2} \rightarrow \cdots \rightarrow V_{-1} \rightarrow W_{0} \rightarrow V_{1} \rightarrow \cdots \rightarrow W_{p-2} \rightarrow V_{p-1},
$$

with all the subbundles of rank 1 except $\operatorname{rk}\left(W_{0}\right)=n=q-p+2$,

$$
\operatorname{deg}\left(V_{k} \text { or } W_{k}\right)=-k(2 g-2) \text { for } 0 \leq k \leq p-2,
$$

and

$$
0<\operatorname{deg}\left(V_{-p+1}\right) \leq(p-1)(2 g-2)
$$

(4) $p \geq 2$ even, $q=p-2+n$ with $n \geq 2$ and

$$
V_{-p+1} \rightarrow W_{-p+2} \rightarrow \cdots \rightarrow V_{-1} \rightarrow W_{0}^{\prime} \rightarrow V_{1} \rightarrow \cdots \rightarrow W_{p-2} \rightarrow V_{p-1}, \quad W_{0}^{\prime \prime},
$$

with all the subbundles of rank 1 except $\operatorname{rk}\left(W_{0}\right)=n=q-p+2$, where $W_{0}=W_{0}^{\prime} \oplus W_{0}^{\prime \prime}$. The first chain is a smooth $\mathrm{SO}_{0}\left(p, p+n^{\prime}\right)$-Higgs bundle with $n^{\prime}=\operatorname{rk}\left(W_{0}^{\prime}\right)$ odd, $W_{0}^{\prime \prime}$ is a smooth $\mathrm{SO}\left(n^{\prime \prime}\right)$-Higgs bundle with $n^{\prime \prime}=\operatorname{rk}\left(W_{0}^{\prime \prime}\right)$ odd,

$$
\operatorname{deg}\left(V_{k} \text { or } W_{k}\right)=-k(2 g-2) \text { for } 0 \leq k \leq p-2,
$$

and

$$
0<\operatorname{deg}\left(V_{-p+1}\right) \leq(p-1)(2 g-2)
$$

(5) $p \geq 3$ odd, $q=p-1+n$ with $n \geq 1$ and

$$
V_{-p+1} \rightarrow W_{-p+2} \rightarrow \cdots \rightarrow W_{-1} \rightarrow V_{0} \rightarrow W_{1} \rightarrow \cdots \rightarrow W_{p-2} \rightarrow V_{p-1}, \quad W_{0}
$$

with all the subbundles in the first chain of rank 1 . The first chain is a smooth $\mathrm{SO}(p, p-1)$-Higgs bundle, $W_{0}$ is a smooth $\mathrm{SO}(n)$-Higgs bundle with $\operatorname{rk}\left(W_{0}\right)=n=$ $q-p+1$ odd, $V_{0} \cong \mathcal{O}$ and

$$
V_{k} \text { or } W_{k} \cong K^{-k} \text { for } 1 \leq k \leq p-1 \text {. }
$$

Note that in the diagrams the arrows represent the Higgs field and are twisted by $K$.

We will prove Theorem 6.10 in several steps. 
Suppose that $\left(V, Q_{V}, W, Q_{W}, \eta\right) \in \mathcal{M}$ is a smooth point which is a critical point of the Hitchin function, then, it is a Hodge bundle and we have decompositions

$$
V=\bigoplus_{m=-r}^{r} V_{m} \text { and } W=\bigoplus_{n=-s}^{s} W_{n},
$$

with $V_{m} \cong\left(V^{*}\right)_{-m}, W_{n} \cong\left(W^{*}\right)_{-n}$ and $\eta_{k}: W_{k} \rightarrow V_{k+1} \otimes K$. Since the point is in particular stable, we can say something more about its decomposition.

Proposition 6.11. If a Hodge bundle $\left(V, Q_{V}, W, Q_{W}, \eta\right) \in \mathcal{M}$ is stable, it has the following structure

$$
\begin{aligned}
& \cdots \rightarrow V_{-2} \rightarrow W_{-1} \rightarrow V_{0} \rightarrow W_{1} \rightarrow V_{2} \rightarrow \cdots \\
& \cdots \rightarrow W_{-2} \rightarrow V_{-1} \rightarrow W_{0} \rightarrow V_{1} \rightarrow W_{2} \rightarrow \cdots
\end{aligned}
$$

with all the bundles and the maps non-zero. There are two chains if $V_{0}$ and $W_{0}$ are non-zero and only one if $V_{0}$ or $W_{0}$ is zero. Each chain ends in a subbundle of $V$ or $W$ depending on the parity of the maximal weight.

Proof. Since $Q_{V}\left(V_{k}, V_{l}\right) \neq 0$ and $Q_{W}\left(W_{k}, W_{l}\right) \neq 0$ if and only if $l=-k$, the subbundles $V_{k}$ and $W_{k}$ are isotropic subbundles of $V$ and $W$ respectively for $k \neq 0$.

Suppose that there is a piece of sequence which is invariant under the Higgs field, for example:

$$
W_{k} \rightarrow V_{k+1} \rightarrow W_{k+2} \rightarrow V_{k+3} \rightarrow \cdots \rightarrow W_{k+r-3} \rightarrow V_{k+r-2} \rightarrow W_{k+r-1} \rightarrow V_{k+r}
$$

and where $V_{0}$ or $W_{0}$ are not included, then

$$
\begin{aligned}
V^{\prime} & =V_{k+1} \oplus V_{k+3} \oplus \cdots \oplus V_{k+r-2} \oplus V_{k+r} \subset V, \\
W^{\prime} & =W_{k} \oplus W_{k+2} \oplus \cdots \oplus W_{k+r-3} \oplus W_{k+r-1} \subset W
\end{aligned}
$$

are isotropic subbundles satisfying $\eta\left(W^{\prime}\right) \subseteq V^{\prime} \otimes K$ and the stability of $\left(V, Q_{V}, W, Q_{W}, \eta\right)$ implies $\operatorname{deg}\left(V^{\prime}\right)+\operatorname{deg}\left(W^{\prime}\right)<0$. Now let

$$
\begin{aligned}
V^{\prime \prime} & =V_{-k-r} \oplus V_{-k-r+2} \oplus \cdots \oplus V_{-k-3} \oplus V_{-k-1} \subset V, \\
W^{\prime \prime} & =W_{-k-r+1} \oplus W_{-k-r+3} \oplus \cdots \oplus W_{-k-2} \oplus W_{-k} \subset W .
\end{aligned}
$$

These are also isotropic subbundles with $\eta\left(W^{\prime \prime}\right) \subseteq V^{\prime \prime} \otimes K$ and since

$$
\operatorname{deg}\left(V^{\prime \prime}\right)+\operatorname{deg}\left(W^{\prime \prime}\right)=-\operatorname{deg}\left(V^{\prime}\right)-\operatorname{deg}\left(W^{\prime}\right)>0,
$$

these subbundles violate the condition of stability of $\left(V, Q_{V}, W, Q_{W}, \eta\right)$. Then, we conclude that in each piece of sequence with non-zero subbundles and non-zero maps there has to be a bundle with weight zero. 
The eigenvalues are integers since in each chain we have always the value zero and consecutive eigenvalues differ by 1 (all maps are non-zero).

Observe that this result is only a first approach to the structure of the smooth points of the moduli and not a full characterization. We will see that stability implies more than the conditions in Proposition 6.11.

In the next proposition we prove the restrictions that the maximal weights of the chains in Proposition 6.11 have to satisfy if the Hodge bundle $\left(V, Q_{V}, W, Q_{W}, \eta\right)$ is a minimum.

Proposition 6.12. Let $\left(V, Q_{V}, W, Q_{W}, \eta\right)$ be an $\mathrm{SO}_{0}(p, q)$-Higgs bundle which represents a smooth point of the moduli space and which is a critical point of the Hitchin function. The point is in particular stable and has the form shown in Proposition 6.11. Let $r$ and $s$ be the maximal weights of the first and the second chain respectively. If $\left(V, Q_{V}, W, Q_{W}, \eta\right)$ falls in one of the following cases, then it does not represent a local minimum of $f$ :

1. The maximal weights coincide.

2. The weights $r$ and $s$ are odd.

3. The weights $r$ and $s$ are even and non-zero.

4. One of the maximal weights is odd and the other is even or zero.

Proof. 1. Suppose that $r=s$ odd, the chains are of the form

$$
\begin{gathered}
W_{-r} \rightarrow V_{-r+1} \rightarrow \cdots \rightarrow W_{-1} \rightarrow V_{0} \rightarrow W_{1} \rightarrow \cdots \rightarrow V_{r-1} \rightarrow W_{r} \\
V_{-r} \rightarrow W_{-r+1} \rightarrow \cdots \rightarrow V_{-1} \rightarrow W_{0} \rightarrow V_{1} \rightarrow \cdots \rightarrow W_{r-1} \rightarrow V_{r}
\end{gathered}
$$

Taking the piece of maximal weight of the complex $\operatorname{ad}(\varphi)$,

$$
C_{2 r}^{\bullet}(E, \varphi): \Lambda^{2} V_{r} \oplus \Lambda^{2} W_{r} \rightarrow 0
$$

we deduce $\operatorname{rk}\left(V_{r}\right)=\operatorname{rk}\left(W_{r}\right)=1$, and considering the piece with weight $2 r-1$ given by

$$
C_{2 r-1}^{\bullet}(E, \varphi): \operatorname{Hom}\left(V_{-r}, V_{r-1}\right) \oplus \operatorname{Hom}\left(W_{-r}, W_{r-1}\right) \rightarrow \operatorname{Hom}\left(W_{-r}, V_{r}\right) \otimes K,
$$

we observe that the isomorphism of the map implies $\operatorname{rk}\left(V_{r-1}\right)+\operatorname{rk}\left(W_{r-1}\right)=1$ which is not possible because in the chains all the subbundles are non-zero, and then $\operatorname{rk}\left(V_{r-1}\right)$, $\operatorname{rk}\left(W_{r-1}\right) \geq 1$.

The argument is exactly the same when $r=s$ is even. 
2. Suppose that $s<r$ and that both weights are odd. The case with $r<s$ and both odd is the same, interchanging $V$ and $W$. We have a Higgs bundle of the form

$$
\begin{gathered}
W_{-r} \rightarrow V_{-r+1} \rightarrow \cdots \rightarrow W_{-1} \rightarrow V_{0} \rightarrow W_{1} \rightarrow \cdots \rightarrow V_{r-1} \rightarrow W_{r} \\
V_{-s} \rightarrow W_{-s+1} \rightarrow \cdots \rightarrow V_{-1} \rightarrow W_{0} \rightarrow V_{1} \rightarrow \cdots \rightarrow W_{s-1} \rightarrow V_{s}
\end{gathered}
$$

Note that $s \leq r-2$. The piece of maximal weight of the deformation complex,

$$
C_{2 r}^{\bullet}(E, \varphi): \Lambda^{2} W_{r} \rightarrow 0
$$

tells us that $\operatorname{rk}\left(W_{r}\right)=1$ and the piece of weight $2 r-2$, which is the next non-zero piece and is given by

$$
C_{2 r-2}^{\bullet}(E, \varphi): \operatorname{Hom}\left(W_{-r}, W_{r-2}\right) \oplus \Lambda^{2} V_{r-1} \rightarrow \operatorname{Hom}\left(W_{-r}, V_{r-1}\right) \otimes K
$$

implies $\operatorname{rk}\left(W_{r-2}\right)=1$ and $\operatorname{rk}\left(V_{r-1}\right)=1$ or 2 . Finally, considering the first piece of the complex with $k$ odd,

$$
C_{r+s-1}^{\bullet}(E, \varphi): \operatorname{Hom}\left(W_{-r}, W_{s-1}\right) \oplus \operatorname{Hom}\left(V_{-r+1}, V_{s}\right) \rightarrow \operatorname{Hom}\left(W_{-r}, V_{s}\right) \otimes K
$$

we obtain a contradiction. If $\operatorname{rk}\left(V_{r-1}\right)=1$, this isomorphism means $\operatorname{rk}\left(W_{s-1}\right)=0$ and if $\operatorname{rk}\left(V_{r-1}\right)=2$, it implies $\operatorname{rk}\left(W_{s-1}\right)+\operatorname{rk}\left(V_{s}\right)=0$. Both situations are not possible.

3. Suppose that $s<r$ and that both weights are even. The case with $r<s$ and both even is the same, interchanging $V$ and $W$. We have a Higgs bundle of the form

$$
\begin{gathered}
V_{-r} \rightarrow W_{-r+1} \rightarrow \cdots \rightarrow W_{-1} \rightarrow V_{0} \rightarrow W_{1} \rightarrow \cdots \rightarrow W_{r-1} \rightarrow V_{r} \\
W_{-s} \rightarrow V_{-s+1} \rightarrow \cdots \rightarrow V_{-1} \rightarrow W_{0} \rightarrow V_{1} \rightarrow \cdots \rightarrow V_{s-1} \rightarrow W_{s}
\end{gathered}
$$

Note that $s \leq r-2$. The piece of maximal weight

$$
C_{2 r}^{\bullet}(E, \varphi): \Lambda^{2} V_{r} \rightarrow 0
$$

tells us that $\operatorname{rk}\left(V_{r}\right)=1$ and the isomorphism

$$
C_{2 r-2}^{\bullet}(E, \varphi): \operatorname{Hom}\left(V_{-r}, V_{r-2}\right) \oplus \Lambda^{2} W_{r-1} \rightarrow \operatorname{Hom}\left(W_{-r+1}, V_{r}\right) \otimes K
$$

implies $\operatorname{rk}\left(V_{r-2}\right)=1$ and $\operatorname{rk}\left(W_{r-1}\right)=1$ or 2 . Finally, considering the first piece of the complex with $k$ odd,

$$
C_{r+s-1}^{\bullet}(E, \varphi): \operatorname{Hom}\left(V_{-r}, V_{s-1}\right) \oplus \operatorname{Hom}\left(W_{-r+1}, W_{s}\right) \rightarrow \operatorname{Hom}\left(W_{-s}, V_{r}\right) \otimes K
$$


we obtain a contradiction with the ranks, as in the previous case. In this proof we are using $r>s \geq 2$. If $s=0$, the bundle $V_{s-1}=V_{-1}$ does not exist, and the first odd complex

$$
C_{r+s-1}^{\bullet}(E, \varphi)=C_{r-1}^{\bullet}(E, \varphi): \operatorname{Hom}\left(W_{-r+1}, W_{0}\right) \rightarrow \operatorname{Hom}\left(W_{0}, V_{r}\right) \otimes K
$$

determines $\operatorname{rk}\left(W_{r-1}\right)=1$, but does not give contradiction.

4. Suppose that $r$ is even and $s$ is odd. The converse case is obtained interchanging $V$ and $W$. We have a Higgs bundle of the form

$$
\begin{gathered}
V_{-r} \rightarrow W_{-r+1} \rightarrow \cdots \rightarrow W_{-1} \rightarrow V_{0} \rightarrow W_{1} \rightarrow \cdots \rightarrow W_{r-1} \rightarrow V_{r}, \\
V_{-s} \rightarrow W_{-s+1} \rightarrow \cdots \rightarrow V_{-1} \rightarrow W_{0} \rightarrow V_{1} \rightarrow \cdots \rightarrow W_{s-1} \rightarrow V_{s} .
\end{gathered}
$$

The first (maximal) odd complex is

$$
C_{r+s}^{\bullet}(E, \varphi): \operatorname{Hom}\left(V_{-r}, V_{s}\right) \rightarrow 0
$$

This isomorphism implies $\operatorname{rk}\left(V_{r}\right) \operatorname{rk}\left(W_{s}\right)=0$ which is not possible.

This proposition tells us that when the subbundles $V_{0}$ and $W_{0}$ are both non-zero, the only possibility for the structure of the minima is the one where the Higgs bundle has one chain with maximal weight even and the other chain with maximal weight equal to zero, that is, with only one subbundle. The rest of the minima has only one chain.

In Propositions 6.13 and 6.14 we will obtain conditions for the ranks of the eigenbundles in the decomposition of a smooth minimum $\left(V, Q_{V}, W, Q_{W}, \eta\right)$.

Proposition 6.13. Let $\left(V, Q_{V}, W, Q_{W}, \eta\right)$ be an $\mathrm{SO}_{0}(p, q)$-Higgs bundle which represents a smooth point of the moduli space and which is a local minimum of the Hitchin function. If this element has $V_{0}$ non-zero and $W_{0}=0$, it has to be in one of the following cases:

1. The maximal weight $r$ is even, that is,

$$
V_{-r} \rightarrow W_{-r+1} \rightarrow \cdots \rightarrow W_{-1} \rightarrow V_{0} \rightarrow W_{1} \rightarrow \cdots \rightarrow W_{r-1} \rightarrow V_{r}
$$

and all the subbundles have rank 1.

2. The maximal weight $r$ is odd, that is,

$$
W_{-r} \rightarrow V_{-r+1} \rightarrow \cdots \rightarrow W_{-1} \rightarrow V_{0} \rightarrow W_{1} \rightarrow \cdots \rightarrow V_{r-1} \rightarrow W_{r}
$$

and all the subbundles have rank 1 except $V_{0}$ that can have any rank. 
3. The maximal weight $r$ is odd and the minimum is of the form

$$
W_{-r} \rightarrow V_{-r+1} \rightarrow \cdots \rightarrow W_{-1} \rightarrow V_{0}^{\prime} \rightarrow W_{1} \rightarrow \cdots \rightarrow V_{r-1} \rightarrow W_{r}, \quad V_{0}^{\prime \prime}
$$

with $V_{0}=V_{0}^{\prime} \oplus V_{0}^{\prime \prime}$ and with all the subbundles of rank 1 except $V_{0}$. The $\mathrm{SO}_{0}(r-1+$ $\left.n^{\prime}, r+1\right)$-Higgs bundle

$$
W_{-r} \rightarrow V_{-r+1} \rightarrow \cdots \rightarrow W_{-1} \rightarrow V_{0}^{\prime} \rightarrow W_{1} \rightarrow \cdots \rightarrow V_{r-1} \rightarrow W_{r}
$$

is smooth, $n^{\prime}=\operatorname{rk}\left(V_{0}^{\prime}\right)$ is odd and $V_{0}^{\prime \prime}$ is a smooth $\mathrm{SO}\left(n^{\prime \prime}\right)$-Higgs bundle with $n^{\prime \prime}=$ $\operatorname{rk}\left(V_{0}^{\prime \prime}\right)$ odd.

If the element has $W_{0}$ non-zero and $V_{0}=0$, the statement is the same, but interchanging $V$ and $W$.

Proof. 1. Let the maximal weight $r$ to be even. We have to determine the rank of $r+1$ subbundles, and there are $r$ complexes $C_{2 r-2 k}^{\bullet}(E, \varphi)$, with $0 \leq k \leq r-1$. The piece of maximal weight of the deformation complex given by

$$
C_{2 r}^{\bullet}(E, \varphi): \Lambda^{2} V_{r} \rightarrow 0
$$

tells us that $\operatorname{rk}\left(V_{r}\right)=1$. The isomorphism for $k=1$,

$$
C_{2 r-2}^{\bullet}(E, \varphi): \operatorname{Hom}\left(V_{-r+2}, V_{r}\right) \oplus \Lambda^{2} W_{r-1} \rightarrow \operatorname{Hom}\left(W_{-r+1}, V_{r}\right) \otimes K
$$

implies

$$
\operatorname{rk}\left(V_{r-2}\right)+\left(\begin{array}{c}
\operatorname{rk}\left(W_{r-1}\right) \\
2
\end{array}\right)=\operatorname{rk}\left(W_{r-1}\right)
$$

and the solutions for this equation $\operatorname{are} \operatorname{rk}\left(V_{r-2}\right)=1$ and $\operatorname{rk}\left(W_{r-1}\right)=1$ or 2 .

If $\operatorname{rk}\left(W_{r-1}\right)=2$, we have

$$
\begin{aligned}
& \begin{array}{llllll}
1 & 2 & 1 & 1 & 2 & 1
\end{array} \\
& V_{-r} \stackrel{\eta_{r-1}^{\top}}{\longrightarrow} W_{-r+1} \stackrel{\eta_{-r+1}}{\longrightarrow} V_{-r+2} \longrightarrow \cdots \longrightarrow V_{r-2} \stackrel{\eta_{-r+1}^{\top}}{\longrightarrow} W_{r-1} \stackrel{\eta_{r-1}}{\longrightarrow} V_{r} .
\end{aligned}
$$

Denote $N=\operatorname{ker} \eta_{r-1}$ and decompose $W_{r-1}=N \oplus M$, then $W_{-r+1}=N^{*} \oplus M^{*}$. We know that $C_{2 r-2}^{\bullet}(E, \varphi)(f, g)=\eta_{r-1} \circ g-f \circ \eta_{-r+1}$ is an isomorphism. Let $f \in$ $\operatorname{Hom}\left(V_{-r+2}, V_{r}\right), g=\left(\begin{array}{cc}0 & g_{1} \\ -g_{1} & 0\end{array}\right) \in \Lambda^{2} W_{r-1}, \eta_{r-1}=(0, a) \in \operatorname{Hom}\left(W_{r-1}, V_{r}\right) \otimes K=$ 
$\operatorname{Hom}\left(N \oplus M, V_{r}\right) \otimes K$ and $\eta_{-r+1}=(b, c) \in \operatorname{Hom}\left(W_{-r+1}, V_{-r+2}\right) \otimes K=\operatorname{Hom}\left(N^{*} \oplus\right.$ $\left.M^{*}, V_{-r+2}\right) \otimes K$. We have

$$
\eta_{r-1} \circ g-f \circ \eta_{-r+1}=(0, a)\left(\begin{array}{cc}
0 & g_{1} \\
-g_{1} & 0
\end{array}\right)-f(b, c)=\left(-a g_{1}-f b, f c\right) .
$$

If $c=0$, the map is not surjective, then $c \neq 0$, and this implies that $\operatorname{Im}\left(\eta_{-r+1}^{\top}\right) \neq \operatorname{ker}\left(\eta_{r-1}\right)$. Then $M=\operatorname{Im}\left(\eta_{-r+1}^{\top}\right)$ and

$$
V_{-r} \stackrel{\eta_{r-1}^{\top}}{\longrightarrow} M^{*} \stackrel{\eta_{-r+1}}{\longrightarrow} V_{-r+2} \longrightarrow \cdots \longrightarrow V_{r-2} \stackrel{\eta_{-r+1}^{\top}}{\longrightarrow} M \stackrel{\eta_{r-1}}{\longrightarrow} V_{r}
$$

$N^{*}$

$N$

with $N$ and $N^{*}$ isotropic. We must have $\operatorname{deg}(N)<0$, but then $N^{*}$ violates the stability, and we conclude that we do not have the case $\operatorname{rk}\left(W_{r-1}\right)=2$.

The piece of the deformation complex for $k=2$,

$$
\begin{aligned}
C_{2 r-4}^{\bullet}(E, \varphi): \quad & \operatorname{Hom}\left(V_{-r+4}, V_{r}\right) \oplus \operatorname{Hom}\left(W_{-r+3}, W_{r-1}\right) \oplus \Lambda^{2} V_{r-2} \\
& \downarrow \\
& \left(\operatorname{Hom}\left(W_{-r+3}, V_{r}\right) \oplus \operatorname{Hom}\left(W_{-r+1}, V_{r-2}\right)\right) \otimes K
\end{aligned}
$$

tells us that

$$
\operatorname{rk}\left(V_{-r+4}\right)+\operatorname{rk}\left(W_{-r+3}\right)=\operatorname{rk}\left(W_{-r+3}\right)+1 .
$$

Then $\operatorname{rk}\left(V_{r-4}\right)=1$.

We conclude the proof of this first part using successively and alternatively the following two statements:

- If $k$ is even and

$$
\begin{aligned}
& \operatorname{rk}\left(W_{r}\right)=\operatorname{rk}\left(W_{r-2}\right)=\cdots=\operatorname{rk}\left(W_{r-2 k+2}\right)=1, \\
& \operatorname{rk}\left(V_{r-1}\right)=\operatorname{rk}\left(V_{r-3}\right)=\cdots=\operatorname{rk}\left(V_{r-k+1}\right)=1
\end{aligned}
$$

then $\operatorname{rk}\left(W_{r-2 k}\right)=1$.

- If $k$ is odd and

$$
\begin{aligned}
& \operatorname{rk}\left(W_{r}\right)=\operatorname{rk}\left(W_{r-2}\right)=\cdots=\operatorname{rk}\left(W_{r-2 k+2}\right)=1, \\
& \operatorname{rk}\left(V_{r-1}\right)=\operatorname{rk}\left(V_{r-3}\right)=\cdots=\operatorname{rk}\left(V_{r-k+2}\right)=1
\end{aligned}
$$

then $\operatorname{rk}\left(W_{r-2 k}\right)=1$ and $\operatorname{rk}\left(V_{r-k}\right)=1$. 
In both cases we use the piece of weight $2 r-2 k$ of the deformation complex, for $1 \leq k \leq r-1$. If $k$ is even, we have

$$
\begin{gathered}
\operatorname{Hom}\left(V_{-r+2 k}, V_{r}\right) \oplus \operatorname{Hom}\left(W_{-r+2 k-1}, W_{r-1}\right) \oplus \cdots \oplus \operatorname{Hom}\left(W_{-r+k+1}, W_{r-k+1}\right) \\
\downarrow \\
\left(\operatorname{Hom}\left(W_{-r+2 k-1}, V_{r}\right) \oplus \operatorname{Hom}\left(W_{-r+1}, V_{r-2 k+2}\right) \oplus \cdots \oplus \operatorname{Hom}\left(W_{-r+k-1}, V_{r-k}\right) \otimes K,\right.
\end{gathered}
$$

where each part of the map has $k$ summands. Simplifying the relations that give this isomorphism for the ranks, we get $\operatorname{rk}\left(V_{r-2 k}\right)=1$.

If $k$ is odd, the piece of weight $2 r-2 k$ is

$$
\begin{array}{r}
\operatorname{Hom}\left(V_{-r+2 k}, V_{r}\right) \oplus \operatorname{Hom}\left(W_{-r+2 k-1}, W_{r-1}\right) \oplus \cdots \oplus \operatorname{Hom}\left(V_{-r+k+1}, V_{r-k+1}\right) \oplus \Lambda^{2} W_{r-k} \\
\downarrow \\
\quad\left(\operatorname{Hom}\left(W_{-r+2 k-1}, V_{r}\right) \oplus \operatorname{Hom}\left(W_{-r+1}, V_{r-2 k+2}\right) \oplus \cdots \oplus \operatorname{Hom}\left(W_{-r+k}, V_{r-k+1}\right) \otimes K,\right.
\end{array}
$$

In terms of the conditions for the ranks, this reduce to the map

$$
\operatorname{Hom}\left(V_{-r+2 k}, V_{r}\right) \oplus \Lambda^{2} W_{r-k} \rightarrow \operatorname{Hom}\left(W_{-r+k}, V_{r-k+1}\right),
$$

which implies

$$
\operatorname{rk}\left(V_{r-2 k}\right)+\left(\begin{array}{c}
\operatorname{rk}\left(W_{r-k}\right) \\
2
\end{array}\right)=\operatorname{rk}\left(W_{r-k}\right) .
$$

The solutions are $\operatorname{rk}\left(V_{r-2 k}\right)=1$ and $\operatorname{rk}\left(W_{r-k}\right)=1$ or 2 , and we remove the case of rank 2 as in the $2 r-2$ case.

We obtain $\operatorname{rk}\left(V_{0}\right)=1$, the last subbundle of $V$, in the step $k=\frac{r}{2}$, and $\operatorname{rk}\left(W_{1}\right)=1$, the small for $W$, in the last step $k=r-1$ which corresponds to the piece of weight 2 of the deformation complex.

2. Let the maximal weight $r$ to be odd. We have to determine the rank of $r+1$ subbundles, and there is $r$ complexes $C_{2 r-2 k}^{\bullet}(E, \varphi)$, with $0 \leq k \leq r-1$. The piece of maximal weight of the deformation complex,

$$
C_{2 r}^{\bullet}(E, \varphi): \Lambda^{2} W_{r} \rightarrow 0
$$

tells us that $\operatorname{rk}\left(W_{r}\right)=1$ and the isomorphism for $k=1$,

$$
C_{2 r-2}^{\bullet}(E, \varphi): \operatorname{Hom}\left(W_{-r+2}, W_{r}\right) \oplus \Lambda^{2} V_{r-1} \rightarrow \operatorname{Hom}\left(W_{-r}, V_{r-1}\right) \otimes K
$$

implies

$$
\operatorname{rk}\left(W_{r-2}\right)+\left(\begin{array}{c}
\operatorname{rk}\left(V_{r-1}\right) \\
2
\end{array}\right)=\operatorname{rk}\left(V_{r-1}\right) .
$$


The solutions are $\operatorname{rk}\left(W_{r-2}\right)=1$ and $\operatorname{rk}\left(V_{r-1}\right)=1$ or 2 . We remove the case of rank 2 as in the previous part of the proposition.

We conclude the proof using successively and alternatively the following two statements:

- If $k$ is even and

$$
\begin{gathered}
\operatorname{rk}\left(V_{r}\right)=\operatorname{rk}\left(V_{r-2}\right)=\cdots=\operatorname{rk}\left(V_{r-2 k+2}\right)=1, \\
\operatorname{rk}\left(W_{r-1}\right)=\operatorname{rk}\left(W_{r-3}\right)=\cdots=\operatorname{rk}\left(W_{r-k+1}\right)=1,
\end{gathered}
$$

then $\operatorname{rk}\left(V_{r-2 k}\right)=1$.

- If $k$ is odd and

$$
\begin{gathered}
\operatorname{rk}\left(V_{r}\right)=\operatorname{rk}\left(V_{r-2}\right)=\cdots=\operatorname{rk}\left(V_{r-2 k+2}\right)=1, \\
\operatorname{rk}\left(W_{r-1}\right)=\operatorname{rk}\left(W_{r-3}\right)=\cdots=\operatorname{rk}\left(W_{r-k+2}\right)=1,
\end{gathered}
$$

then $\operatorname{rk}\left(V_{r-2 k}\right)=1$ and $\operatorname{rk}\left(W_{r-k}\right)=1$.

In both cases we use the piece of weight $2 r-2 k$ of the deformation complex, for $1 \leq k \leq r-1$. If $k$ is even, we have

$$
\begin{gathered}
\operatorname{Hom}\left(W_{-r+2 k}, W_{r}\right) \oplus \operatorname{Hom}\left(V_{-r+2 k-1}, V_{r-1}\right) \oplus \cdots \oplus \operatorname{Hom}\left(V_{-r+k+1}, V_{r-k+1}\right) \\
\downarrow \\
\left(\operatorname{Hom}\left(W_{-r}, V_{r-2 k+1}\right) \oplus \operatorname{Hom}\left(W_{-r+2 k-2}, V_{r-1}\right) \oplus \cdots \oplus \operatorname{Hom}\left(W_{-r+k}, V_{r-k+1}\right) \otimes K,\right.
\end{gathered}
$$

where each part of the map has $k$ summands. Simplifying the relations that give this isomorphism for the ranks, we get $\operatorname{rk}\left(W_{r-2 k}\right)=1$.

If $k$ is odd, the piece of weight $2 r-2 k$ is the complex

$$
\begin{array}{r}
\operatorname{Hom}\left(W_{-r+2 k}, W_{r}\right) \oplus \operatorname{Hom}\left(V_{-r+2 k-1}, V_{r-1}\right) \oplus \cdots \oplus \operatorname{Hom}\left(W_{-r+k+1}, W_{r-k+1}\right) \oplus \Lambda^{2} V_{r-k} \\
\downarrow \\
\left(\operatorname{Hom}\left(W_{-r}, V_{r-2 k+1}\right) \oplus \operatorname{Hom}\left(W_{-r+2 k-2}, V_{r-1}\right) \oplus \cdots \oplus \operatorname{Hom}\left(W_{-r+k-1}, V_{r-k}\right) \otimes K,\right.
\end{array}
$$

In terms of the conditions for the ranks, this reduce to the map

$$
\operatorname{Hom}\left(W_{-r+2 k}, W_{r}\right) \oplus \Lambda^{2} V_{r-k} \rightarrow \operatorname{Hom}\left(W_{-r+k-1}, V_{r-k}\right)
$$

which implies

$$
\operatorname{rk}\left(W_{r-2 k}\right)+\left(\begin{array}{c}
\operatorname{rk}\left(V_{r-k}\right) \\
2
\end{array}\right)=\operatorname{rk}\left(V_{r-k}\right) .
$$


The solutions are $\operatorname{rk}\left(W_{r-2 k}\right)=1$ and $\operatorname{rk}\left(V_{r-k}\right)=1$ or 2 , and we remove the case of rank 2 as in the case of weight $2 r-2$.

We obtain $\operatorname{rk}\left(W_{1}\right)=1$, the last subbundle of $W$, in the step $k=\frac{r-1}{2}$, and $\operatorname{rk}\left(V_{2}\right)=1$, in the penultimate step $k=r-2$ which corresponds to the piece of weight 4 of the deformation complex. The last piece of the complex does not give new information and the rank of $V_{0}$ remains not fixed.

3. Suppose that the maximal weight $r$ of the chain is odd and that we have a splitting $V_{0}=V_{0}^{\prime} \oplus V_{0}^{\prime \prime}$ such that the Higgs bundle decomposes as

$$
W_{-r} \rightarrow V_{-r+1} \rightarrow \cdots \rightarrow W_{-1} \rightarrow V_{0}^{\prime} \rightarrow W_{1} \rightarrow \cdots \rightarrow V_{r-1} \rightarrow W_{r}, \quad V_{0}^{\prime \prime} .
$$

We do not have this situation in 1 . because in this case $\operatorname{rk}\left(V_{0}\right)=1$. Since $\left(V, Q_{V}, W, Q_{W}, \eta\right)$ is stable, Lemmas 3.19 and 3.20 tells us that

$$
W_{-r} \rightarrow V_{-r+1} \rightarrow \cdots \rightarrow W_{-1} \rightarrow V_{0}^{\prime} \rightarrow W_{1} \rightarrow \cdots \rightarrow V_{r-1} \rightarrow W_{r}
$$

is a stable $\mathrm{SO}_{0}\left(r-1+n^{\prime}, r+1\right)$-Higgs bundle and $V_{0}^{\prime \prime}$ is stable as $\mathrm{SO}\left(n^{\prime \prime}\right)$-Higgs bundle.

On the other hand, the $\mathrm{SO}_{0}\left(r-1+n^{\prime}+n^{\prime \prime}, r+1\right)$-Higgs bundle $\left(V, Q_{V}, W, Q_{W}, \eta\right)$ is also simple. The only way to obtain this is taking a simple $\mathrm{SO}_{0}\left(r-1+n^{\prime}, r+1\right)$-Higgs bundle with $n^{\prime}$ odd and a simple $\mathrm{SO}\left(n^{\prime \prime}\right)$-Higgs bundle with $n^{\prime \prime}$ odd. Since $n^{\prime}$ is odd, $r-1+n^{\prime}$ is odd and the only automorphism of the simple $\mathrm{SO}_{0}\left(r-1+n^{\prime}, r+1\right)$-Higgs bundle is the identity. Analogously, since $n^{\prime \prime}$ is odd, the only automorphism of the simple $\mathrm{SO}\left(n^{\prime \prime}\right)$-Higgs bundle is the identity.

Proposition 6.14. Let $\left(V, Q_{V}, W, Q_{W}, \eta\right)$ be an $\mathrm{SO}_{0}(p, q)$-Higgs bundle which represents a smooth point of the moduli space and which is a local minimum of the Hitchin function. If $V_{0}$ and $W_{0}$ are non-zero, the Higgs bundle has to be of the form

$$
V_{-r} \rightarrow W_{-r+1} \rightarrow \cdots \rightarrow W_{-1} \rightarrow V_{0} \rightarrow W_{1} \rightarrow \cdots \rightarrow W_{r-1} \rightarrow V_{r}, \quad W_{0}
$$

with $r>0$ even and with all the subbundles in the first chain of rank 1 . The first chain is a smooth $\mathrm{SO}_{0}(r+1, r)$-Higgs bundle and $W_{0}$ is a smooth $\mathrm{SO}\left(n_{0}\right)$-Higgs bundle with $n_{0}=\operatorname{rk}\left(W_{0}\right)$ odd.

It is also possible to have a Higgs bundle with the structure obtained interchanging $V$ and $W$.

Proof. First of all, using Proposition 6.12, we know that if $V_{0}$ and $W_{0}$ are non-zero, the point has to fit in with the structure

$$
V_{-r} \rightarrow W_{-r+1} \rightarrow \cdots \rightarrow W_{-1} \rightarrow V_{0} \rightarrow W_{1} \rightarrow \cdots \rightarrow W_{r-1} \rightarrow V_{r}, \quad W_{0}
$$


(or the other interchanging $V$ and $W$ ), with $r>0$ and even.

The piece of maximal weight of the deformation complex,

$$
C_{2 r}^{\bullet}(E, \varphi): \Lambda^{2} V_{r} \rightarrow 0
$$

tells us that $\operatorname{rk}\left(V_{r}\right)=1$. We obtain the result using alternatively the following two steps, for any $0 \leq k \leq r-2$ and even:

1. If $\operatorname{rk}\left(V_{r-k}\right)=1$, then $\operatorname{rk}\left(W_{r-k-1}\right)=1$.

It is enough to consider the complex

$$
C_{r-k-1}^{\bullet}(E, \varphi): \operatorname{Hom}\left(W_{0}, W_{r-k-1}\right) \rightarrow \operatorname{Hom}\left(W_{0}, V_{r-k}\right) \otimes K .
$$

2. If $\operatorname{rk}\left(V_{r-k}\right)=1$, then $\operatorname{rk}\left(V_{r-k-2}\right)=1$.

We have the isomorphism

$$
C_{2 r-k-2}^{\bullet}(E, \varphi): \operatorname{Hom}\left(V_{-r}, V_{r-k-2}\right) \oplus \cdots \rightarrow\left(\operatorname{Hom}\left(W_{-r+k+1}, V_{r}\right) \oplus \ldots\right) \otimes K,
$$

where the dots are replacing homomorphisms between subbundles that we already know that have rank 1 . This implies that the ranks have to satisfy

$$
\frac{k+2}{2}-1+\operatorname{rk}\left(V_{r-k-2}\right)=\frac{k+2}{2},
$$

and hence $\operatorname{rk}\left(V_{r-k-2}\right)=1$.

Finally, we need $\mathrm{rk}\left(W_{0}\right)$ odd to guarantee simplicity. We do not have any similar condition in the $\mathrm{SO}_{0}(r+1, r)$-Higgs bundle because $q^{\prime}=r$ is odd.

Finally, in Propositions 6.15, 6.16 and 6.17 we will obtain conditions for the degree of the eigenbundles in the decomposition of a smooth minimum $\left(V, Q_{V}, W, Q_{W}, \eta\right)$.

Proposition 6.15. Let $\left(V, Q_{V}, W, Q_{W}, \eta\right)$ be an $\mathrm{SO}_{0}(p, q)$-Higgs bundle which represents a smooth point of the moduli space and which is a local minimum of the Hitchin function. If this Higgs bundle is of the type 1. of Proposition 6.13, that is, it has maximal weight $r$ even and is of the form

$$
V_{-r} \rightarrow W_{-r+1} \rightarrow \cdots \rightarrow W_{-1} \rightarrow V_{0} \rightarrow W_{1} \rightarrow \cdots \rightarrow W_{r-1} \rightarrow V_{r},
$$

with all the subbundles of rank 1 , then

$$
V_{k} \text { or } W_{k} \cong K^{-k} \text { for } 0 \leq k \leq r-1
$$

and

$$
0<\operatorname{deg}\left(V_{-r}\right) \leq r(2 g-2) .
$$


Proof. The existence of the non-zero maps

$$
V_{0} \stackrel{\eta_{-1}^{\top}}{\longrightarrow} W_{1} \stackrel{\eta_{1}}{\longrightarrow} \cdots \stackrel{\eta_{-r+1}^{\top}}{\longrightarrow} W_{r-1} \stackrel{\eta_{r-1}}{\longrightarrow} V_{r}
$$

gives the following relations

$$
\begin{aligned}
\eta_{-1}^{\top} & \rightsquigarrow \operatorname{deg}\left(W_{1}\right) \geq-(2 g-2), \\
\eta_{1} & \rightsquigarrow \operatorname{deg}\left(V_{2}\right) \geq \operatorname{deg}\left(W_{1}\right)-(2 g-2) \geq-2(2 g-2), \\
\eta_{-3}^{\top} & \rightsquigarrow \quad \cdots \\
\eta_{3} & \rightsquigarrow \quad \cdots \\
& \cdots \\
\eta_{-k}^{\top} & \rightsquigarrow \operatorname{deg}\left(W_{k}\right) \geq \operatorname{deg}\left(V_{k-1}\right)-(2 g-2) \geq-k(2 g-2), \\
\eta_{k} & \rightsquigarrow \operatorname{deg}\left(V_{k+1}\right) \geq \operatorname{deg}\left(W_{k}\right)-(2 g-2) \geq-(k+1)(2 g-2), \\
& \cdots \\
\eta_{-r+1}^{\top} & \rightsquigarrow \operatorname{deg}\left(W_{r-1}\right) \geq \operatorname{deg}\left(V_{r-2}\right)-(2 g-2) \geq-(r-1)(2 g-2), \\
\eta_{r-1} & \rightsquigarrow \operatorname{deg}\left(V_{r}\right) \geq \operatorname{deg}\left(W_{r-1}\right)-(2 g-2) \geq-r(2 g-2),
\end{aligned}
$$

because we have a non-zero global section $\eta_{k} \in H^{0}\left(W_{k}^{*} \otimes V_{k+1} \otimes K\right)$ if and only if $-\operatorname{deg}\left(W_{k}\right)+\operatorname{deg}\left(V_{k+1}\right)+2 g-2 \geq 0$, and the analogous for $\eta_{k}^{\top}$. (Since $\left.V_{0} \cong V_{0}^{*}, \operatorname{deg} V_{0}=0\right)$. Then we have the inequalities

$$
\operatorname{deg}\left(V_{k} \text { or } W_{k}\right) \geq-k(2 g-2) \text { for } 1 \leq k \leq r \text {. }
$$

The criterion of Proposition 6.9, tells us that the $r-1$ complexes $C_{2 r-2 k}^{\bullet}(E, \varphi), 1 \leq$ $k \leq r-1$ are isomorphisms. This give us the following system of $r-1$ equations for the degrees of $V_{k}\left(k\right.$ even) and $W_{k}(k$ odd $)$ for $1 \leq k \leq r-1$ :

$1 \leq k<\frac{r}{2}:$

$$
\operatorname{deg}\left(V_{r-2 k}\right)=\operatorname{deg}\left(V_{r-k} \text { or } W_{r-k}\right)+k(2 g-2) .
$$

$k=\frac{r}{2}:$

$$
\operatorname{deg}\left(W_{\frac{r}{2}}\right)=-\frac{r}{2}(2 g-2)
$$

$\frac{r}{2}<k \leq r-1:$

$$
-\operatorname{deg}\left(V_{2 k-r}\right)=\operatorname{deg}\left(V_{r-k} \text { or } W_{r-k}\right)+k(2 g-2) .
$$

Solving the system we obtain $\operatorname{deg}\left(V_{k}\right.$ or $\left.W_{k}\right)=-k(2 g-2)$ for $1 \leq k \leq r-1$.

The bundle $V_{0}$ is an special orthogonal bundle, and then $V_{0} \cong \mathcal{O}$. In

$$
V_{0} \rightarrow W_{1} \otimes K \rightarrow \cdots \rightarrow W_{r-1} \otimes K^{r-1} \rightarrow V_{r} \otimes K^{r}
$$


the arrows are all non-zero homomorphisms between line bundles of the same degree, then they are isomorphisms. The first one, $k=1$, give us $\mathcal{O} \cong W_{1} \otimes K$, that is, $W_{1} \cong K^{-1}$, and successively we obtain $V_{k}$ or $W_{k} \cong K^{-k}$ for $1 \leq k \leq r-1$.

We can not determine the degree of $V_{r}$, but we know that

$$
0>\operatorname{deg}\left(V_{r}\right) \geq-r(2 g-2)
$$

where the first inequality is given by stability, or equivalently

$$
0<\operatorname{deg}\left(V_{-r}\right) \leq r(2 g-2)
$$

Proposition 6.16. Let $\left(V, Q_{V}, W, Q_{W}, \eta\right)$ be an $\mathrm{SO}_{0}(p, q)$-Higgs bundle which represents a smooth point of the moduli space and which is a local minimum of the Hitchin function. If this Higgs bundle is of the type 2. of Proposition 6.13, that is, it has maximal weight $r$ odd and is of the form

$$
W_{-r} \rightarrow V_{-r+1} \rightarrow \cdots \rightarrow W_{-1} \rightarrow V_{0} \rightarrow W_{1} \rightarrow \cdots \rightarrow V_{r-1} \rightarrow W_{r},
$$

with all the subbundles of rank 1 except $V_{0}$ that can have any rank, then

$$
\operatorname{deg}\left(V_{k} \text { or } W_{k}\right)=-k(2 g-2) \text { for } 0 \leq k \leq r-1,
$$

and

$$
0<\operatorname{deg}\left(W_{-r}\right) \leq r(2 g-2)
$$

The same result is verified for a minimum of type 3. of Proposition 6.13.

Proof. The existence of the non-zero Higgs fields gives again a sequence

$$
\begin{aligned}
\eta_{-1}^{\top} & \rightsquigarrow \operatorname{deg}\left(W_{1}\right) \geq-(2 g-2), \\
\eta_{1} & \rightsquigarrow \operatorname{deg}\left(V_{2}\right) \geq \operatorname{deg}\left(W_{1}\right)-(2 g-2) \geq-2(2 g-2), \\
& \cdots \\
\eta_{-r+2}^{\top} & \rightsquigarrow \operatorname{deg}\left(W_{r-2}\right) \geq \operatorname{deg}\left(V_{r-3}\right)-(2 g-2) \geq-(r-2)(2 g-2), \\
\eta_{r-2} & \rightsquigarrow \operatorname{deg}\left(V_{r-1}\right) \geq \operatorname{deg}\left(W_{r-2}\right)-(2 g-2) \geq-(r-1)(2 g-2), \\
\eta_{-r}^{\top} & \rightsquigarrow \operatorname{deg}\left(W_{r}\right) \geq \operatorname{deg}\left(V_{r-1}\right)-(2 g-2) \geq-r(2 g-2) .
\end{aligned}
$$

and

$$
\operatorname{deg}\left(V_{k} \text { or } W_{k}\right) \geq-k(2 g-2) \text { for } 1 \leq k \leq r .
$$

The criterion of Proposition 6.9, tells us that the $r-1$ complexes $C_{2 r-2 k}^{\bullet}(E, \varphi), 1 \leq$ $k \leq r-1$ are isomorphisms. This give us the following system of $r-1$ equations for the degrees of $W_{k}\left(k\right.$ even) and $V_{k}(k$ odd $)$ for $1 \leq k \leq r-1$ : 
$1 \leq k \leq \frac{r-1}{2}:$

$$
\operatorname{deg}\left(W_{r-2 k}\right)=\operatorname{deg}\left(V_{r-k} \text { or } W_{r-k}\right)+k(2 g-2) .
$$

$\frac{r+1}{2} \leq k \leq r-1:$

$$
-\operatorname{deg}\left(W_{2 k-r}\right)=\operatorname{deg}\left(V_{r-k} \text { or } W_{r-k}\right)+k(2 g-2) \text {. }
$$

Solving the system we obtain $\operatorname{deg}\left(V_{k}\right.$ or $\left.W_{k}\right)=-k(2 g-2)$ for $1 \leq k \leq r-1$.

We can not conclude $V_{0} \cong \mathcal{O}$ and $V_{k}$ or $W_{k} \cong K^{-k}$ for $1 \leq k \leq r-1$ as in Proposition 6.15 because $\operatorname{rk}\left(V_{0}\right)$ is arbitrary. It is possible only when $\operatorname{rk}\left(V_{0}\right)=1$.

We can not determine the degree of $W_{r}$, but we get

$$
0<\operatorname{deg}\left(W_{-r}\right) \leq r(2 g-2)
$$

The proof for the type 3 . is the same.

Proposition 6.17. Let $\left(V, Q_{V}, W, Q_{W}, \eta\right)$ be an $\mathrm{SO}_{0}(p, q)$-Higgs bundle which represents a smooth point of the moduli space and which is a local minimum of the Hitchin function. If this Higgs bundle is of the form of Proposition 6.14, that is, it decomposes as

$$
V_{-r} \rightarrow W_{-r+1} \rightarrow \cdots \rightarrow W_{-1} \rightarrow V_{0} \rightarrow W_{1} \rightarrow \cdots \rightarrow W_{r-1} \rightarrow V_{r}, \quad W_{0},
$$

with $r>0$ even and with all the subbundles in the first chain of rank 1 , where the first chain is a smooth $\mathrm{SO}_{0}(r+1, r)$-Higgs bundle and $W_{0}$ is a smooth $\mathrm{SO}\left(n_{0}\right)$-Higgs bundle with $n_{0}=\operatorname{rk}\left(W_{0}\right)$ odd, then $V_{0} \cong \mathcal{O}$ and

$$
V_{k} \text { or } W_{k} \cong K^{-k} \text { for } 1 \leq k \leq r
$$

Proof. The existence of the non-zero Higgs fields gives the same inequalities as in Proposition 6.15 .

$$
\operatorname{deg}\left(V_{k} \text { or } W_{k}\right) \geq-k(2 g-2) \text { for } 1 \leq k \leq r .
$$

Using the even complexes $C_{2 r-2 k}^{\bullet}(E, \varphi), 1 \leq k \leq r-1$ we obtain

$$
\operatorname{deg}\left(V_{k} \text { or } W_{k}\right)=-k(2 g-2) \text { for } 0 \leq k \leq r-1
$$

and we determine the degree of $V_{r}$ using the first odd complex

$$
C_{r-1}^{\bullet}(E, \varphi): \operatorname{Hom}\left(W_{0}, W_{r-1}\right) \rightarrow \operatorname{Hom}\left(W_{0}, V_{r}\right) \otimes K
$$

which implies

$$
\operatorname{deg}\left(V_{r}\right)=\operatorname{deg}\left(W_{r-1}\right)-(2 g-2)=-r(2 g-2) .
$$

We conclude as in Proposition 6.15 
Now we are in position to prove the main result.

Proof of Theorem 6.10: These five types of minima are the minima that we have described in the previous results.

The minima of type (1) correspond to the minima of Proposition 6.15. We have interchanged $V$ and $W$ to obtain $p \leq q$ and we have changed the notation using $p$ instead of $r$.

The minima of type (2) correspond to the minima of Proposition 6.16 with $n=1$ and $p=r$. And in the types (3) and (4) we have the minima of Proposition 6.16 with $n \geq 2$. We have interchanged $V$ and $W$ and we have taken $p=r+1$.

Finally, minima of type (5) are the minima of Proposition 6.17 with $p=r+1$. 


\section{$7 \quad$ Non-smooth minima}

Theorem 3.7 gives us a decomposition of a polystable $\mathrm{SO}_{0}(p, q)$-Higgs bundles as a sum of stable $G_{i}$-Higgs bundles, where $G_{i}$ is one of the following groups: $\mathrm{SO}_{0}\left(p_{i}, q_{i}\right), \mathrm{U}\left(p_{i}, q_{i}\right)$, $\mathrm{SO}\left(n_{i}\right)$ or $\mathrm{U}\left(n_{i}\right)$. The following result tells us that, in fact, any polystable $\mathrm{SO}_{0}(p, q)$-Higgs bundles can be decomposed as a sum of smooth $G_{i}$-Higgs bundles.

Proposition 7.1. Let $\left(V, Q_{V}, W, Q_{W}, \eta\right)$ be a polystable $\mathrm{SO}_{0}(p, q)$-Higgs bundle. There is a decomposition, unique up to reordering, of this Higgs bundle in a sum of smooth $G_{i}$-Higgs bundles, where $G_{i}=\mathrm{SO}_{0}\left(p_{i}, q_{i}\right), \mathrm{U}\left(p_{i}, q_{i}\right), \mathrm{SO}\left(n_{i}\right)$ or $\mathrm{U}\left(n_{i}\right)$.

Proof. The starting point is Theorem 3.7 .

A stable $\mathrm{U}(n)$-Higgs bundle and a stable $\mathrm{U}(p, q)$-Higgs bundle represent smooth points in the moduli spaces of $\mathrm{U}(n)$-Higgs bundles and $\mathrm{U}(p, q)$-Higgs bundles respectively.

A stable $\mathrm{SO}(n)$-Higgs bundle is smooth if and only if it is stable and simple. On the other hand, any stable $\mathrm{SO}(n)$-Higgs bundle which is not simple can be expressed, using Theorem 3.18, as a direct sum of smooth $\mathrm{SO}\left(n_{i}\right)$-Higgs bundles.

Finally, as we know from Corollary 3.15, a stable $\mathrm{SO}_{0}(p, q)$-Higgs bundle represents a smooth point of the moduli space if and only if it is simple, but if a stable $\operatorname{SO}_{0}(p, q)$ Higgs bundle is non-simple, Theorem 3.21 tells us that it decomposes as a sum of smooth $\mathrm{SO}_{0}\left(p_{i}, q_{i}\right)$ and $\mathrm{SO}\left(n_{i}\right)$-Higgs bundles.

Since the Hitchin function $f$ is additive with respect to this direct sum, if the polystable Higgs bundle is a minimum, each Higgs bundle in the decomposition (with smooth summands) has to be a minimum on the corresponding moduli space $\mathcal{M}\left(G_{i}\right)$ and a minimum as $\mathrm{SO}_{0}\left(p_{i}, q_{i}\right)$-Higgs bundle.

The summands corresponding to $\mathrm{U}\left(n_{i}\right)$ or $\mathrm{SO}\left(n_{i}\right)$-Higgs bundles are minima, because the Higgs field is zero in both cases.

A $\mathrm{U}\left(p_{i}, q_{i}\right)$-Higgs bundle $\left(V_{i}, W_{i}, \beta_{i}, \gamma_{i}\right)$ is a minimum if and only if $\beta_{i}=0$ or $\gamma_{i}=0$. Then, the corresponding $\mathrm{SO}_{0}\left(2 p_{i}, 2 q_{i}\right)$-Higgs bundle $\left(V_{i} \oplus V_{i}^{*},\langle\cdot, \cdot\rangle, W_{i} \oplus W_{i}^{*},\langle\cdot, \cdot\rangle, \eta_{i}=\right.$ $\left.\beta_{i}+\gamma_{i}^{t}\right)$ has $\eta_{i}=\beta_{i}$ or $\eta_{i}=\gamma_{i}^{t}$. Suppose that $\gamma_{i}=0$, then we have $\eta_{i}=\beta_{i}: W_{i} \rightarrow V_{i} \otimes K$ and the corresponding $\eta_{i}^{t}: V_{i}^{*} \rightarrow W_{i}^{*} \otimes K$. The analogous if $\beta_{i}=0$.

A smooth $\mathrm{SO}_{0}\left(p_{i}, q_{i}\right)$-Higgs bundle is a minimum if it is in one of the cases of Theorem 6.10 . 
If an $\mathrm{SO}_{0}(p, q)$-Higgs bundle $(E, \varphi)$ is stable but it is not simple or it is strictly polystable, Corollary 3.15 tells us that this Higgs bundle does not represent a smooth point on the moduli space of polystable $\mathrm{SO}_{0}(p, q)$-Higgs bundles, and then, we can not use the criterion of Proposition 6.9 to determine if $(E, \varphi)$ is a minimum of the Hitchin function. But Hitchin proves in $[32$ that even when the Higgs bundle is not smooth, if $\mathbb{H}^{1}\left(C_{k}^{\bullet}(E, \varphi)\right)=0$ for all $k>0$, then $(E, \varphi)$ is a local minimum. Hence, we can include in type (4) of Theorem 6.10 the cases with $n^{\prime \prime}=\operatorname{rk}\left(W_{0}^{\prime \prime}\right)$ even that we have removed to guarantee the simplicity of the total $\mathrm{SO}_{0}(p, q)$-Higgs bundle in the case $n^{\prime \prime}>2$ and even, and also the stability in the case $n^{\prime \prime}=2$. Analogously, we can include in type (5) of Theorem 6.10 the cases with $\operatorname{rk}\left(W_{0}\right)$ even. We conjecture that these are all the possible stable minima in $\mathcal{M}\left(\mathrm{SO}_{0}(p, q)\right)$.

Theorem 7.2. Let $\left(V, Q_{V}, W, Q_{W}, \eta\right)$ be a polystable $\mathrm{SO}_{0}(p, q)$-Higgs bundle, with $p \leq q$, which is a local minimum of the Hitchin function. If the Higgs field is not equal to zero, the minimum is in one of the following cases:

(1) $p \geq 2$ even, $q=p+1$ and

$$
W_{-p} \rightarrow V_{-p+1} \rightarrow \cdots \rightarrow V_{-1} \rightarrow W_{0} \rightarrow V_{1} \rightarrow \cdots \rightarrow V_{p-1} \rightarrow W_{p}
$$

with all subbundles of rank 1 ,

$$
V_{k} \text { or } W_{k} \cong K^{-k} \text { for } 0 \leq k \leq p-1
$$

and

$$
0<\operatorname{deg}\left(W_{-p}\right) \leq p(2 g-2)
$$

(2) $p \geq 1$ odd, $q=p+1$ and

$$
W_{-p} \rightarrow V_{-p+1} \rightarrow \cdots \rightarrow W_{-1} \rightarrow V_{0} \rightarrow W_{1} \rightarrow \cdots \rightarrow V_{p-1} \rightarrow W_{p}
$$

with all subbundles of rank 1 ,

$$
V_{k} \text { or } W_{k} \cong K^{-k} \text { for } 0 \leq k \leq p-1
$$

and

$$
0<\operatorname{deg}\left(W_{-p}\right) \leq p(2 g-2)
$$

(3) $p \geq 2$ even, $q=p-2+n$ with $n \geq 2$ and

$$
V_{-p+1} \rightarrow W_{-p+2} \rightarrow \cdots \rightarrow V_{-1} \rightarrow W_{0} \rightarrow V_{1} \rightarrow \cdots \rightarrow W_{p-2} \rightarrow V_{p-1}
$$


with all the subbundles of rank 1 except $\operatorname{rk}\left(W_{0}\right)=n=q-p+2$,

$$
\operatorname{deg}\left(V_{k} \text { or } W_{k}\right)=-k(2 g-2) \text { for } 0 \leq k \leq p-2,
$$

and

$$
0<\operatorname{deg}\left(V_{-p+1}\right) \leq(p-1)(2 g-2)
$$

(4) $p \geq 2$ even, $q=p-2+n$ with $n \geq 2$ and

$$
V_{-p+1} \rightarrow W_{-p+2} \rightarrow \cdots \rightarrow V_{-1} \rightarrow W_{0}^{\prime} \rightarrow V_{1} \rightarrow \cdots \rightarrow W_{p-2} \rightarrow V_{p-1}, \quad W_{0}^{\prime \prime},
$$

with all the subbundles of rank 1 except $\operatorname{rk}\left(W_{0}\right)=n=q-p+2$, where $W_{0}=W_{0}^{\prime} \oplus W_{0}^{\prime \prime}$. The first chain is a smooth $\mathrm{SO}_{0}\left(p, p+n^{\prime}\right)$-Higgs bundle with $n^{\prime}=\operatorname{rk}\left(W_{0}^{\prime}\right), W_{0}^{\prime \prime}$ is a smooth $\mathrm{SO}\left(n^{\prime \prime}\right)$-Higgs bundle with $n^{\prime \prime}=\operatorname{rk}\left(W_{0}^{\prime \prime}\right)$,

$$
\operatorname{deg}\left(V_{k} \text { or } W_{k}\right)=-k(2 g-2) \text { for } 0 \leq k \leq p-2,
$$

and

$$
0<\operatorname{deg}\left(V_{-p+1}\right) \leq(p-1)(2 g-2) .
$$

(5) $p \geq 3$ odd, $q=p-1+n$ with $n \geq 1$ and

$$
V_{-p+1} \rightarrow W_{-p+2} \rightarrow \cdots \rightarrow W_{-1} \rightarrow V_{0} \rightarrow W_{1} \rightarrow \cdots \rightarrow W_{p-2} \rightarrow V_{p-1}, \quad W_{0}
$$

with all the subbundles in the first chain of rank 1 . The first chain is a smooth $\mathrm{SO}(p, p-1)$-Higgs bundle, $W_{0}$ is a smooth $\mathrm{SO}(n)$-Higgs bundle with $\operatorname{rk}\left(W_{0}\right)=n=$ $q-p+1, V_{0} \cong \mathcal{O}$ and

$$
V_{k} \text { or } W_{k} \cong K^{-k} \text { for } 1 \leq k \leq p-1 \text {. }
$$

Note that in the diagrams the arrows represent the Higgs field and are twisted by $K$.

Since the condition

$$
\mathbb{H}^{1}\left(C_{-}^{\bullet}(E, \varphi)\right)=\bigoplus_{k>0} \mathbb{H}^{1}\left(C_{k}^{\bullet}(E, \varphi)\right) \neq 0
$$

does not imply that the point is not a minimum, to rule out a stable but non-simple or a strictly polystable element as minimum it is necessary the following result (see Hitchin $[32, \S 8])$.

Proposition 7.3. Let $(E, \varphi)$ be a polystable $\mathrm{SO}_{0}(p, q)$-Higgs bundle whose isomorphism class is fixed under the circle action. Suppose that $\left\{\left(E_{t}, \varphi_{t}\right)\right\}_{t}$ is a family of polystable $\mathrm{SO}_{0}(p, q)$-Higgs bundles deforming $(E, \varphi)$ and that the corresponding infinitesimal deformation is a non-zero element of $\mathbb{H}^{1}\left(C_{-}^{\bullet}(E, \varphi)\right)$, then $(E, \varphi)$ is not a local minimum. 
Proof. Let $(A, \varphi) \in \mathcal{M}_{c}^{\text {Hit }}\left(\mathrm{SO}_{0}(p, q)\right)$ be the solution corresponding to the Higgs bundle $(E, \varphi)$. Remember that $A$ is a connection on $E_{\mathrm{SO}(p) \times \mathrm{SO}(q)}$ and $\varphi \in \Omega^{1,0}\left(E_{\mathrm{SO}(p) \times \mathrm{SO}(q)}\left(\mathfrak{m}^{\mathbb{C}}\right)\right)$. If $(A, \varphi)$ is a fixed point of the circle action, there is a 1-parameter family of gauge transformations $g(\theta)=\left(g_{1}(\theta), g_{2}(\theta)\right) \in \mathcal{G}\left(E_{\mathrm{SO}(p) \times \mathrm{SO}(q)}\right)$ such that

$$
\left(A, e^{i \theta} \varphi\right)=g(\theta) \cdot(A, \varphi)=(g(\theta) \cdot A, g(\theta) \cdot \varphi) .
$$

This family is generated by an infinitesimal gauge transformation $\psi=\left(\psi_{1}, \psi_{2}\right)$ which is covariantly constant, $d_{A_{1}}\left(\psi_{1}\right)=d_{A_{2}}\left(\psi_{2}\right)=0$, and with $[\psi, \varphi]=i \varphi$. Denote $\left(A_{\theta}, \varphi_{\theta}\right)=$ $g(\theta) \cdot(A, \varphi)$, then $\left(A_{0}, \varphi_{0}\right)=(A, \varphi)$, and consider the corresponding infinitesimal variation $(\dot{A}, \dot{\varphi}) \in \mathbb{H}^{1}\left(C^{\bullet}(E, \varphi)\right)$.

We know from [32, $\S 8]$ that if the circle acts with weights $m$ and $n$ on $(\dot{A}, \dot{\varphi})$ with $m>0$ or $n>1$, the pair $(A, \varphi)$ is not a local minimum of $f$. This is proved by computing $\ddot{f}$ and showing that for these values of $m$ and $n$ it is negative in certain directions. The condition $m>0$ or $n>1$ is equivalent to $(\dot{A}, \dot{\varphi}) \in \mathbb{H}^{1}\left(C_{-}^{\bullet}(E, \varphi)\right)$ and we conclude.

Technical problems arise when we try to prove Theorem 7.2 applying Proposition 7.3 which do not enable us to conclude using these methods. However, we give a full description of the polystable minima of the Hitchin function in $\mathcal{M}(p, q)$ in some particular cases and solve the problem of counting the number of connected components.

\subsection{Minima in $\mathcal{M}\left(\mathrm{SO}_{0}(3,3)\right)$}

From Theorem 6.10 we have that the only smooth minimum of the Hitchin function with Higgs field non-equal to zero in the moduli space of polystable $\mathrm{SO}_{0}(3,3)$-Higgs bundles is the minimum of type (5) with $p=3$ and $n=\operatorname{rk}\left(W_{0}\right)=1$, that is,

$$
V_{-2} \rightarrow W_{-1} \rightarrow V_{0} \rightarrow W_{1} \rightarrow V_{2}, \quad W_{0},
$$

with $V_{0} \cong W_{0} \cong \mathcal{O}, V_{1} \cong K^{-1}$ and $W_{2} \cong K^{-2}$. From Section 8.4, we also know that this minimum is the minimum in the Hitchin component.

There is an isomorphism between the moduli space of polystable $\mathrm{SO}_{0}(3,3)$-Higgs bundles and the moduli space of polystable $\mathrm{SL}(4, \mathbb{R})$-Higgs bundles which is described in Section 5.4. The result given by Theorem 7.2 agrees with the results about $\operatorname{SL}(4, \mathbb{R})$ Higgs bundles proved by Hitchin in [32]. In this paper he proved that the minima in $\mathcal{M}(\mathrm{SL}(4, \mathbb{R}))$ are those with Higgs field equal to zero together with the minimum of the Hitchin component, which is:

$$
K^{3 / 2} \rightarrow K^{1 / 2} \rightarrow K^{-1 / 2} \rightarrow K^{-3 / 2}
$$


Using the isomorphism

$$
\mathcal{M}(\mathrm{SL}(4, \mathbb{R})) \rightarrow \mathcal{M}\left(\mathrm{SO}_{0}(3,3)\right)
$$

of Section 5.4 that is, taking $\Lambda^{2}\left(K^{3 / 2} \oplus K^{1 / 2} \oplus K^{-1 / 2} \oplus K^{-3 / 2}\right)=V \oplus W$ and considering the corresponding orthogonal form and Higgs field, it is easy to check that the minimum in the Hitchin component of $\mathcal{M}(\mathrm{SL}(4, \mathbb{R}))$ goes to the minimum in the Hitchin component of $\mathcal{M}\left(\mathrm{SO}_{0}(3,3)\right)$.

\subsection{Minima in $\mathcal{M}\left(\mathrm{SO}_{0}(1, n)\right)$ with $n$ odd}

An $\mathrm{SO}_{0}(1, n)$-Higgs bundle is a tuple $\left(V, Q_{V}, W, Q_{W}, \eta\right)$, where $\left(V, Q_{V}\right) \cong \mathcal{O},\left(W, Q_{W}\right)$ is a principal $\mathrm{SO}(n, \mathbb{C})$-bundle and $\eta: W \rightarrow \mathcal{O} \otimes K$.

In this section we describe the minima of the Hitchin function in the moduli space of $\mathrm{SO}_{0}(1, n)$-Higgs bundles with $n$ odd.

We first apply Theorem 6.10 to see which are the smooth minima of the Hitchin function in $\mathcal{M}\left(\mathrm{SO}_{0}(1, n)\right)$. For $n=2$, the only smooth minima with Higgs field non-equal to zero are the minima of type $(2)$ (with $p=1$ ), that is, those of the form

$$
W_{-1} \rightarrow V \rightarrow W_{1}
$$

with $0<\operatorname{deg}\left(W_{-1}\right) \leq 2 g-2$. For $n=1$ or $n>2$ there are no smooth minima with non-zero Higgs field.

Theorem 7.4. There are no minima of the Hitchin function with non-zero Higgs field in the moduli space of polystable $\mathrm{SO}_{0}(1, n)$-Higgs bundles with $n$ odd.

Proof. Using Theorem 6.10 we have already seen that there are no smooth minima with non-zero Higgs field in $\mathcal{M}\left(\mathrm{SO}_{0}(1, n)\right)$ if $n \neq 2$, then it is true for $n$ odd.

1. If $\left(V, Q_{V}, W, Q_{W}, \eta\right)$ is a stable but non-simple $\mathrm{SO}_{0}(1, n)$-Higgs bundle ( $n$ odd) with $\eta \neq 0$ which is a fixed point of the circle action, using Theorem 3.21 and Proposition 6.6. we obtain that it decomposes as a sum of a smooth minimum in $\mathcal{M}\left(\mathrm{SO}_{0}(1,2)\right)$ of the form

$$
W_{-1} \rightarrow V \rightarrow W_{1} \text {, with } V \cong \mathcal{O} \text { and } 0<\operatorname{deg}\left(W_{-1}\right) \leq 2 g-2 \text {, }
$$

together with a sum of $\mathrm{SO}\left(n_{i}\right)$-Higgs bundles $W^{i}$ with $n_{i}=\operatorname{rk}\left(W^{i}\right) \neq 2$ where at least one has rank $n_{i}$ even. The first summand is necessary to guarantee the condition $\eta \neq 0$ and the condition for the rank $n_{i}$ to be even determines the non-simplicity of $\left(V, Q_{V}, W, Q_{W}, \eta\right)$.

Since $n$ is odd, $n-2$ is also odd, and hence, at least one of the $\mathrm{SO}\left(n_{i}\right)$-Higgs bundles $W^{i}$ in the decomposition has rank $n_{i}$ odd. If we consider this summand together with the 
one of the form $W_{-1} \rightarrow V \rightarrow W_{1}$, we obtain a smooth $\mathrm{SO}\left(1, n_{i}+2\right)$-Higgs bundle which, by Theorem 6.10, is not a minimum (observe that $W^{i} \cong\left(W^{i}\right)^{*}$ and then it has weight zero). This implies that $\left(V, Q_{V}, W, Q_{W}, \eta\right)$ is not a minimum and we conclude.

2. If $\left(V, Q_{V}, W, Q_{W}, \eta\right)$ is a strictly polystable $\mathrm{SO}_{0}(1, n)$-Higgs bundle ( $n$ odd) with $\eta \neq 0$ which is a fixed point of the circle action, it decomposes as a sum of a smooth minimum in $\mathcal{M}\left(\mathrm{SO}_{0}(1,2)\right)$ of the form

$$
W_{-1} \rightarrow V \rightarrow W_{1} \text {, with } V \cong \mathcal{O} \text { and } 0<\operatorname{deg}\left(W_{-1}\right) \leq 2 g-2 \text {, }
$$

together with a sum of $\mathrm{SO}\left(n_{i}\right)$-Higgs bundles and at least one $\mathrm{U}\left(n_{i}\right)$-Higgs bundle. The existence of at least one $\mathrm{U}\left(n_{i}\right)$-Higgs bundle in the decomposition is necessary to guarantee the strict polystability of $\left(V, Q_{V}, W, Q_{W}, \eta\right)$. Since $n$ is odd, $n-2$ is also odd, and since

$$
\mathrm{U}\left(n_{i}\right) \hookrightarrow \mathrm{SO}\left(2 n_{i}\right) \hookrightarrow \mathrm{SO}_{0}(1, n-2)
$$

with $2 n_{i}$ even, there is at least one $\mathrm{SO}\left(n_{i}\right)$-Higgs bundle $W^{i}$ in the decomposition with $n_{i}$ odd.

As in the stable but non-simple case, if we consider this summand $W^{i}$ together with the one of the form $W_{-1} \rightarrow V \rightarrow W_{1}$, we obtain a smooth $\mathrm{SO}\left(1, n_{i}+2\right)$-Higgs bundle which is not a minimum and we conclude.

Using the characterization of the minima given by Theorem 7.4 solve the problem of counting the connected components of the moduli space $\mathcal{M}\left(\mathrm{SO}_{0}(1, n)\right)$ with $n$ odd.

Theorem 7.5. The moduli space of $\mathrm{SO}_{0}(1, n)$-Higgs bundles with $n>1$ and odd has 2 connected components.

Proof. The topological invariant associated to an $\mathrm{SO}_{0}(1, n)$-Higgs bundle $\left(\mathcal{O}, W, Q_{W}, \eta\right)$ with $n \geq 3$ is the Stiefel-Whitney class $w_{2} \in \pi_{1}(\operatorname{SO}(n, \mathbb{C})) \cong \mathbb{Z}_{2}=\{0,1\}$. Theorem 7.4 tells us that there are no minima of the Hitchin function with non-zero Higgs field, and then $\mathcal{M}\left(\mathrm{SO}_{0}(1, n)\right)$ is the disjoint union of the moduli spaces $\mathcal{M}_{0}\left(\mathrm{SO}_{0}(1, n)\right)$ and $\mathcal{M}_{1}\left(\mathrm{SO}_{0}(1, n)\right)$, which are connected. 


\section{The Hitchin component}

The general theory summarized in Section 8.2 is due to Hitchin [31]. Defining a section of the Hitchin map of a complex Lie group $G$, he gives an isomorphism between certain vector space of dimension $2(g-1) \operatorname{dim} G$ and a connected component of the moduli space of polystable $G^{s}$-Higgs bundles, where $G^{s}$ denotes the split real form of $G$. This component is called the Hitchin component, and there are $2^{2 g}$ connected components in $\mathcal{M}\left(G^{s}\right)$ isomorphic to it, which is the number of possible elections of the square $K^{1 / 2}$.

In Sections 8.5 and 8.4 we apply these results to the orthogonal split real forms $\mathrm{SO}_{0}(n, n)$ and $\mathrm{SO}_{0}(n, n+1)$.

\subsection{Hitchin component for $\operatorname{SL}(2, \mathbb{R})$}

The simplest case to consider is the complex Lie group $\operatorname{SL}(2, \mathbb{C})$ and its split real form $\mathrm{SL}(2, \mathbb{R})$. The Lie algebra $\mathfrak{s l}(2, \mathbb{C})$ has rank 1 and the algebra of invariant polynomials on it is generated by an element $p_{2}$ of degree 2 obtained from the characteristic polynomial

$$
\operatorname{det}(x-A)=x^{2}+p_{2}(A)
$$

of a trace free matrix $A$. We are going to define a section of the Hitchin map

$$
\begin{aligned}
p: \mathcal{M} & \rightarrow H^{0}\left(K^{2}\right), \\
(A, \varphi) & \mapsto p_{2}(\varphi),
\end{aligned}
$$

where $\mathcal{M}=\mathcal{M}(\mathrm{SL}(2, \mathbb{C}))$ denotes the moduli space of polystable $\mathrm{SL}(2, \mathbb{C})$-Higgs bundles. This section $s: H^{0}\left(K^{2}\right) \rightarrow \mathcal{M}$ will give an isomorphism between the vector space $H^{0}\left(K^{2}\right)$ and a connected component of the moduli space $\mathcal{M}(\mathrm{SL}(2, \mathbb{R})) \subset \mathcal{M}$ of polystable $\mathrm{SL}(2, \mathbb{R})$ Higgs bundles. To construct the section $s$, we consider the elements

$$
\left\langle x=\left(\begin{array}{cc}
1 & 0 \\
0 & -1
\end{array}\right), e=\left(\begin{array}{ll}
0 & 1 \\
0 & 0
\end{array}\right), \tilde{e}=\left(\begin{array}{ll}
0 & 0 \\
1 & 0
\end{array}\right)\right\rangle \cong \mathfrak{s l}(2, \mathbb{C}),
$$

that satisfy

$$
[x, e]=2 e,[x, \tilde{e}]=-2 \tilde{e} \text { and }[e, \tilde{e}]=x,
$$

where $x$ is an element of the Cartan subalgebra (a semisimple element) and $e, \tilde{e}$ are nilpotent. In the vector bundle $K^{1 / 2} \oplus K^{-1 / 2}$ we can consider the orthogonal structure $Q=\left(\begin{array}{cc}0 & 1 \\ 1 & 0\end{array}\right)$, and if $\alpha \in H^{0}\left(K^{2}\right)$, the Higgs field $\varphi=\tilde{e}-\alpha e=\left(\begin{array}{cc}0 & -\alpha \\ 1 & 0\end{array}\right)$ is 
symmetric with respect to $Q$, then the pair $\left(K^{1 / 2} \oplus K^{-1 / 2}, \varphi=\tilde{e}-\alpha e=\left(\begin{array}{cc}0 & -\alpha \\ 1 & 0\end{array}\right)\right)$, is an $\mathrm{SL}(2, \mathbb{R})$-Higgs bundle. We define the section by

$$
s(\alpha)=\left(K^{1 / 2} \oplus K^{-1 / 2}, \varphi=\left(\begin{array}{cc}
0 & -\alpha \\
1 & 0
\end{array}\right)\right) .
$$

That is, the set

$$
\left\{\left(K^{1 / 2} \oplus K^{-1 / 2}, \varphi=\left(\begin{array}{cc}
0 & -\alpha \\
1 & 0
\end{array}\right)\right)\right\}_{\alpha \in H^{2}\left(K^{2}\right)}
$$

forms a connected component of $\mathcal{M}(\mathrm{SL}(2, \mathbb{R}))$ called the Hitchin component. This component has dimension $6 g-6$ and there are $2^{2 g}$ connected components isomorphic to this one, which is the number of possible elections of the square root $K^{1 / 2}$.

\subsection{The general construction}

Let $G$ be a complex simple Lie group and $G^{s}$ be its split real form. If the Lie algebra $\mathfrak{g}$ has rank $l$ and $p_{1}, \ldots, p_{l}$ of degrees $m_{1}+1, \ldots, m_{l}+1$ form a basis of the algebra of invariant polynomials on the Lie algebra $\mathfrak{g}$, we can consider the holomorphic map

$$
p: \mathcal{M} \rightarrow \bigoplus_{i=1}^{l} H^{0}\left(K^{m_{i}+1}\right)
$$

defined by

$$
p(A, \varphi)=\left(p_{1}(\varphi), \ldots, p_{l}(\varphi)\right),
$$

where $\mathcal{M}=\mathcal{M}(G)$ denotes the moduli space of polystable $G$-Higgs bundles, or equivalently, the space of solutions to Hitchin's equations. The map $p$ is called the Hitchin map and the elements $m_{1}, \ldots, m_{l}$, which are fundamental invariants of the Lie algebra, are the exponents of $\mathfrak{g}$.

We are going to define a section of the Hitchin map that will give an isomorphism between the vector space $\bigoplus_{i=1}^{l} H^{0}\left(K^{m_{i}+1}\right)$ and a connected component of the moduli space $\mathcal{M}\left(G^{s}\right) \subset \mathcal{M}$ of polystable $G^{s}$-Higgs bundles.

A nilpotent element $e \in \mathfrak{g}$ is called regular if its centralizer is $l$-dimensional. If $\mathfrak{c}$ is a Cartan subalgebra of $\mathfrak{g}$ and $\Delta$ are the corresponding roots, the element

$$
e=\sum_{\alpha \in \Delta^{+}} c_{\alpha} X_{\alpha},
$$


where $X_{\alpha}$ is a root vector for $\alpha$, is always nilpotent and it is regular if $c_{\alpha} \neq 0$ for $\alpha \in \Pi$. Any nilpotent element can be embedded in a 3-dimensional simple subalgebra $\langle x, e, \tilde{e}\rangle \cong \mathfrak{s l}(2, \mathbb{C})$, where $x$ is semisimple, $e$ and $\tilde{e}$ are nilpotent, and they satisfy

$$
[x, e]=2 e ; \quad[x, \tilde{e}]=-2 \tilde{e} ; \quad[e, \tilde{e}]=x .
$$

The adjoint action

$$
\langle x, e, \tilde{e}\rangle \cong \mathfrak{s l}(2, \mathbb{C}) \rightarrow \operatorname{End}(\mathfrak{g})
$$

of this subalgebra breaks up the Lie algebra $\mathfrak{g}$ as a direct sum of irreducible representations

$$
\mathfrak{g}=\bigoplus_{i=1}^{l} V_{i}
$$

with $\operatorname{dim}\left(V_{i}\right)=2 m_{i}+1$. That is, each $V_{i}$ is the irreducible representation $S^{2 m_{i}} \mathbb{C}^{2}$ of $\mathfrak{s l}(2, \mathbb{C})$, where $\mathbb{C}^{2}$ is the standard representation, and the eigenvalues of ad $x$ on $V_{i}$ are $-2 m_{i},-2 m_{i}+2, \ldots, 2 m_{i}-2,2 m_{i}$. The highest weight vector of $V_{i}$, defined as a vector $e_{i} \in V_{i}$ that is eigenvector for the action of $x$ and is in the kernel of ad(e), has eigenvalue $2 m_{i}$ for ad $x$. We take $V_{1}=\langle x, e, \tilde{e}\rangle$ and $e=e_{1}$.

nent by

Given $\left(\alpha_{1}, \ldots, \alpha_{l}\right) \in \bigoplus_{i=1}^{l} H^{0}\left(K^{m_{i}+1}\right)$, we define the Higgs field in the Hitchin compo-

$$
\varphi=\tilde{e}_{1}+\alpha_{1} e_{1}+\ldots+\alpha_{l} e_{l}
$$

To know how the bundle in the Hitchin component looks like, we have to understand the representation

$$
\mathfrak{s l}(2, \mathbb{C}) \rightarrow \mathfrak{g} .
$$

In the classical cases, we can embed $\mathfrak{g}$ into the corresponding general linear Lie algebra $\mathfrak{g l}(m, \mathbb{C})$. In this cases, the Lie algebra $\mathfrak{g}$ is equal to $\operatorname{End}(R), \Lambda^{2}(R)$ or $S^{2}(R) \ldots$ etc. for some representation $R$ of $\mathfrak{s l}(2, \mathbb{C})$. On the other hand, we have the decomposition of $\mathfrak{g}$ as representation of $\mathfrak{s l}(2, \mathbb{C})$,

$$
\mathfrak{s l}(2, \mathbb{C}) \rightarrow \operatorname{End}(\mathfrak{g})=\operatorname{End}\left(S^{2 m_{1}} \mathbb{C}^{2}+\ldots+S^{2 m_{l}} \mathbb{C}^{2}\right) .
$$

Then,

$$
S^{2 m_{1}} \mathbb{C}^{2}+\ldots+S^{2 m_{l}} \mathbb{C}^{2}=\operatorname{End}(R), \Lambda^{2}(R) \text { or } S^{2}(R) \ldots \text { etc. }
$$

and using Clebsch-Gordan formulas (see remark below), we can determine $R$ as a sum of symmetric powers of the standard representation of $\mathfrak{s l}(2, \mathbb{C})$. Transforming the vector bundle $K^{1 / 2} \oplus K^{-1 / 2}$ under this sum we obtain the corresponding vector bundle $\mathbb{E}$ in the Hitchin component of $\mathcal{M}\left(G^{s}\right)$. We will see examples in the following subsections to illustrate this process. 
Theorem 8.1. With the previous notations, if $\left(\alpha_{1}, \ldots, \alpha_{l}\right) \in \bigoplus_{i=1}^{l} H^{0}\left(K^{m_{i}+1}\right)$,

$$
s\left(\alpha_{1}, \ldots, \alpha_{l}\right)=\left(\mathbb{E}, \varphi=\tilde{e}_{1}+\sum_{i=1}^{l} \alpha_{i} e_{i}\right)
$$

defines a section of the Hitchin map $p$ and an isomorphism from the vector space

$$
\bigoplus_{i=1}^{l} H^{0}\left(K^{m_{i}+1}\right)
$$

to a connected component of the moduli space $\mathcal{M}\left(G^{s}\right)$.

This connected component is called the Hitchin component and has dimension $2(g-1) \operatorname{dim} G^{s}$.

Remark: Clebsch-Gordan formulas.

Let $S^{n}=S^{n} \mathbb{C}^{2}$ be the irreducible representation of dimension $n+1$ of the Lie algebra $\mathfrak{s l}(2, \mathbb{C})$. We have that $S^{n} \otimes S^{n}=S^{2}\left(S^{n}\right) \oplus \Lambda^{2}\left(S^{n}\right)$. On the other hand,

$$
S^{2}\left(S^{n}\right)=\bigoplus_{\alpha \geq 0} S^{2 n-4 \alpha} \text { and } \Lambda^{2}\left(S^{n}\right)=S^{2}\left(S^{n-1}\right)=\bigoplus_{\alpha \geq 0} S^{2 n-2-4 \alpha} .
$$

For a sum of irreducible representations, we know that

$$
\Lambda^{2}\left(S^{n} \oplus S^{m}\right)=\bigoplus_{a+b=2} \Lambda^{a} S^{n} \otimes \Lambda^{b} S^{m} .
$$

Using this formulas we can determine the representation $R$ (such that $\mathfrak{g}=\operatorname{End}(R), \Lambda^{2}(R)$, $S^{2}(R) \ldots$ etc.) from the decomposition of $\mathfrak{g}$ in sum of irreducible representations of $\mathfrak{s l}(2, \mathbb{C})$.

\subsection{Hitchin component for $\operatorname{SL}(n, \mathbb{R})$}

Consider the special general case $\operatorname{SL}(n, \mathbb{R})$ which is the split real form of $\operatorname{SL}(n, \mathbb{C})$. The Lie algebra $\mathfrak{s l}(n, \mathbb{C})$ has rank $n-1$ and a basis for the invariant polynomials on $\mathfrak{s l}(n, \mathbb{C})$ is provided by the coefficients of the characteristic polynomial of a trace-free matrix,

$$
\operatorname{det}(x-A)=x^{n}+p_{1}(A) x^{n-2}+\ldots+p_{n-1}(A),
$$

where $\operatorname{deg}\left(p_{i}\right)=i+1$. We can consider the Hitchin map

$$
p: \mathcal{M}(\mathrm{SL}(n, \mathbb{C})) \rightarrow \bigoplus_{i=1}^{n-1} H^{0}\left(K^{i+1}\right)
$$


defined by

$$
p(A, \varphi)=\left(p_{1}(\varphi), \ldots, p_{n-1}(\varphi)\right)
$$

where $\mathcal{M}(\operatorname{SL}(n, \mathbb{C}))$ is the moduli space of polystable $\operatorname{SL}(n, \mathbb{C})$-Higgs bundles.

We are going to define a section of this map that will give an isomorphism between the vector space $\bigoplus_{i=1}^{n-1} H^{0}\left(K^{i+1}\right)$ and a connected component of the moduli space $\mathcal{M}(\operatorname{SL}(n, \mathbb{R})) \subset$ $\mathcal{M}(\mathrm{SL}(n, \mathbb{C}))$ of polystable $\mathrm{SL}(n, \mathbb{R})$-Higgs bundles.

A nilpotent element $e \in \mathfrak{s l}(n, \mathbb{C})$ is called regular if its centralizer is $(n-1)$-dimensional. In $\mathfrak{s l}(n, \mathbb{C})$ a regular nilpotent element is conjugate to an element

$$
e=\sum_{\alpha \in \Pi} c_{\alpha} X_{\alpha}
$$

where $\Pi=\left\{\alpha_{i}=e_{i}-e_{i+1}, 1 \leq i \leq n-1\right\}$ and $X_{\alpha_{i}}=E_{i, i+1}$ is a root vector for $\alpha_{i}$. Any nilpotent element can be embedded in a 3 -dimensional simple subalgebra $\langle x, e, \tilde{e}\rangle \cong$ $\mathfrak{s l}(2, \mathbb{C})$, where $x$ is semisimple, $e$ and $\tilde{e}$ are nilpotent, and they satisfy

$$
[x, e]=2 e ; \quad[x, \tilde{e}]=-2 \tilde{e} ; \quad[e, \tilde{e}]=x
$$

The adjoint action

$$
\langle x, e, \tilde{e}\rangle \cong \mathfrak{s l}(2, \mathbb{C}) \rightarrow \operatorname{End}(\mathfrak{s l}(n, \mathbb{C}))
$$

of this subalgebra breaks up the Lie algebra $\mathfrak{s l}(n, \mathbb{C})$ as a direct sum of irreducible representations

$$
\mathfrak{s l}(n, \mathbb{C})=\bigoplus_{i=1}^{n-1} V_{i}
$$

with $\operatorname{dim}\left(V_{i}\right)=2 i+1$. That is, each $V_{i}$ is the irreducible representation $S^{2 i} \mathbb{C}^{2}$, where $\mathbb{C}^{2}$ is the standard representation of $\mathfrak{s l}(2, \mathbb{C})$, and the eigenvalues of ad $x$ on $V_{i}$ are $-2 i,-2 i+$ $2, \ldots, 2 i-2,2 i$.

The highest weight vector of $V_{i}$, defined as a vector $e_{i} \in V_{i}$ that is eigenvector for the action of $x$ and is in the kernel of $\operatorname{ad}(e)$, has eigenvalue $2 i$ for $\operatorname{ad} x$. We take $V_{1}=\langle x, e, \tilde{e}\rangle$ and $e=e_{1}$.

nent by

Given $\left(\alpha_{1}, \ldots, \alpha_{n-1}\right) \in \bigoplus_{i=1}^{n-1} H^{0}\left(K^{i+1}\right)$, we define the Higgs field in the Hitchin compo-

$$
\varphi=\tilde{e}_{1}+\alpha_{1} e_{1}+\ldots+\alpha_{n-1} e_{n-1},
$$

and the vector bundle is given by

$$
S^{n-1}\left(K^{-1 / 2} \oplus K^{1 / 2}\right)=K^{-(n-1) / 2} \oplus K^{-(n-3) / 2} \oplus \cdots \oplus K^{(n-3) / 2} \oplus K^{(n-1) / 2} .
$$


The field $\varphi$ is given in the following order of the basis, $K^{(n-1) / 2} \oplus K^{(n-3) / 2} \oplus \cdots \oplus K^{-(n-3) / 2} \oplus$ $K^{-(n-1) / 2}$. The minimum in the Hitchin component is given by the values $\alpha_{1}=\cdots=$ $\alpha_{n-1}=0$, then it is the pair

$$
\left(K^{(n-1) / 2} \oplus K^{(n-3) / 2} \oplus \cdots \oplus K^{-(n-3) / 2} \oplus K^{-(n-1) / 2}, \varphi=\tilde{e}\right),
$$

that is

$$
K^{(n-1) / 2} \rightarrow K^{(n-3) / 2} \rightarrow \cdots \rightarrow K^{-(n-3) / 2} \rightarrow K^{-(n-1) / 2} .
$$

Note that in this diagrams the maps are twisted by $K$ and $K^{m} \rightarrow K^{m-1}$ must be interpreted as $K^{m} \rightarrow K^{m-1} \otimes K=K^{m}$.

Example 8.2. Hitchin component of the moduli space of polystable SL(4, $\mathbb{R})$-Higgs bundles.

The sets of positive and simple roots of $\mathfrak{s l}(4, \mathbb{C})$ are

$$
\Delta^{+}=\left\{e_{1}-e_{2}, e_{1}-e_{3}, e_{1}-e_{4}, e_{2}-e_{3}, e_{2}-e_{4}, e_{3}-3_{4}\right\}
$$

We know that $e=\left(\begin{array}{ccc}c_{1,2} & c_{1,3} & c_{1,4} \\ & c_{2,3} & c_{2,4} \\ & & c_{3,4}\end{array}\right)$, with $c_{1,2}, c_{2,3}, c_{3,4} \neq 0$, is a regular nilpotent element. If $x=\left(\begin{array}{cccc}h_{1} & & & \\ & h_{2} & & \\ & & h_{3} & \\ & & & h_{4}\end{array}\right)$ is in the Cartan subalgebra, it satisfies $[x, e]=2 e$ if and only if $h_{1}=3 / 2, h_{2}=1 / 2, h_{3}=-1 / 2, h_{4}=-3 / 2$ and $c_{1,3}=c_{1,4}=c_{2,4}=0$. So finally we can consider

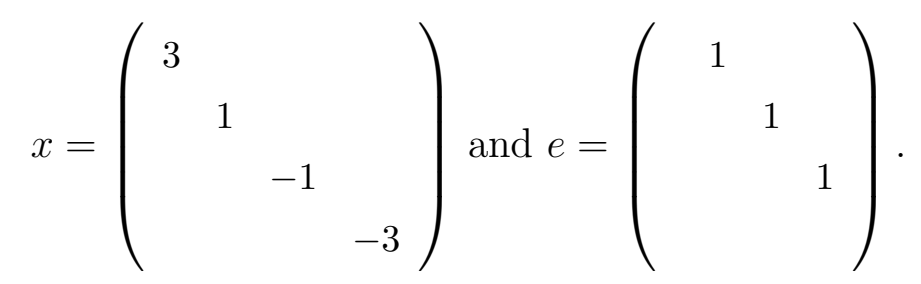

We know that the conditions $[x, \tilde{e}]=-2 \tilde{e}$ and $[e, \tilde{e}]=x$ determine $\tilde{e}$. If

$$
X=\left(\begin{array}{llll}
x_{1,1} & x_{1,2} & x_{1,3} & x_{1,4} \\
x_{2,1} & x_{2,2} & x_{2,3} & x_{2,4} \\
x_{3,1} & x_{3,2} & x_{3,3} & x_{3,4} \\
x_{4,1} & x_{4,2} & x_{4,3} & x_{4,4}
\end{array}\right) \in \mathfrak{s l}(4, \mathbb{C})
$$


the adjoint action of the semisimple element $x$ on $X$ is given by

$$
\operatorname{ad}(x)(X)=\left(\begin{array}{cccc}
0 & 2 x_{1,2} & 4 x_{1,3} & 6 x_{1,4} \\
-2 x_{2,1} & 0 & 2 x_{2,3} & 4 x_{2,4} \\
-4 x_{3,1} & -2 x_{3,2} & 0 & 2 x_{3,4} \\
-6 x_{4,1} & -4 x_{4,2} & -2 x_{4,3} & 0
\end{array}\right)
$$

and the conditions imply $\tilde{e}=\left(\begin{array}{ccc}3 & & \\ & 4 & \\ & & -3\end{array}\right)$. Thus, we obtain the 3-dimensional subalgebra

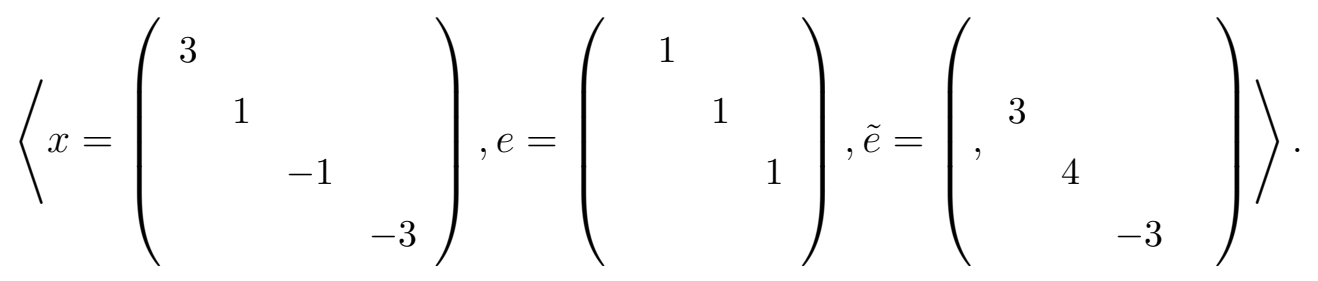

We have a basis of the invariant polynomials $\left\{p_{1}, p_{2}, p_{3}\right\}$ of degrees 2,3 and 3 respectively and the decomposition

$$
\mathfrak{s l}(4, \mathbb{C})=V_{1} \oplus V_{2} \oplus V_{3}=S^{2} \mathbb{C}^{2} \oplus S^{4} \mathbb{C}^{2} \oplus S^{6} \mathbb{C}^{2},
$$

of the Lie algebra $\mathfrak{s l}(4, \mathbb{C})$ as a sum of irreducible representations of $\mathfrak{s l}(2, \mathbb{C})$. The highest weight vector of $V_{2}$ is of the form $e_{2}=\left(\begin{array}{cc}x_{1,3} & \\ & x_{2,4} \\ & \end{array}\right)$ and it is in the kernel of $\operatorname{ad}(e)$ if

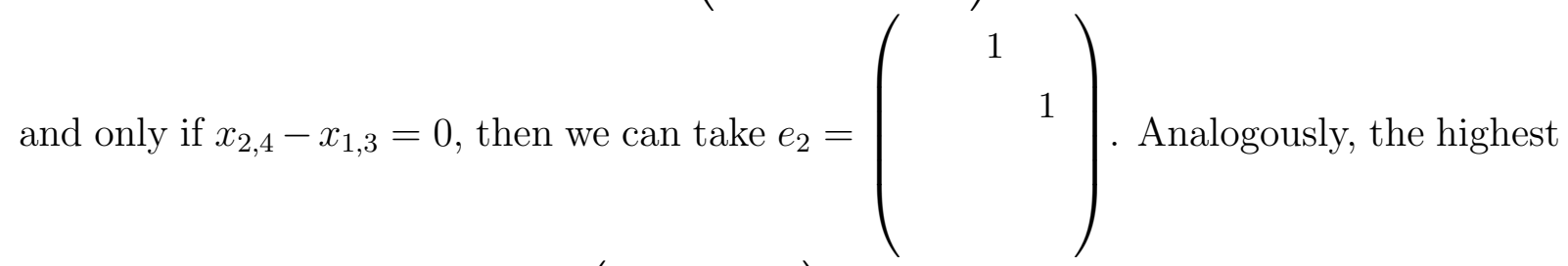
vector of $V_{3}$ is of the form $e_{3}=\left(\begin{array}{c}x_{1,4} \\ \end{array}\right)$ and it is in the $\operatorname{kernel}$ of $\operatorname{ad}(e)$ for all the possible values of $X_{1,4}$, then we can take $e_{2}=\left(\begin{array}{r}1 \\ \end{array}\right)$. Finally, we define the Higgs 
field in the Hitchin component by

$$
\varphi=\left(\begin{array}{cccc} 
& \alpha_{1} & \alpha_{2} & \alpha_{3} \\
3 & & \alpha_{1} & \alpha_{2} \\
& 4 & & \alpha_{1} \\
& & -3 &
\end{array}\right) .
$$

The vector bundle in the Hitchin component is given by

$$
S^{3}\left(K^{1 / 2} \oplus K^{-1 / 2}\right)=K^{3 / 2} \oplus K^{1 / 2} \oplus K^{-1 / 2} \oplus K^{-3 / 2} .
$$

The minimum in the Hitchin component is given by the values $\alpha_{1}=\cdots=\alpha_{n-1}=0$, then it is the pair

$$
\left(K^{3 / 2} \oplus K^{1 / 2} \oplus K^{-1 / 2} \oplus K^{1 / 2}, \varphi=\tilde{e}\right),
$$

that is

$$
K^{3 / 2} \rightarrow K^{1 / 2} \rightarrow \cdots \rightarrow K^{-1 / 2} \rightarrow K^{-3 / 2}
$$

\subsection{Hitchin component for $\mathrm{SO}_{0}(n, n)$}

The Lie group $\mathrm{SO}_{0}(n, n)$ is the split real form of $\mathrm{SO}(2 n, \mathbb{C})$. A basis of the invariant polynomials on $\mathfrak{s o}(2 n, \mathbb{C})$ is provided by the coefficients of the characteristic polynomial of a skew-symmetric matrix, which is of the form

$$
\operatorname{det}(x-A)=x^{2 n}+p_{1}(A) x^{2 n-2}+\ldots+p_{n}(A),
$$

where $\operatorname{deg} p_{i}=2 i$. The polynomial $p_{n}=\operatorname{det} A$ of degree $2 n$ is the square of the Pfaffian polynomial $p_{n}^{\prime}$, which has degree $n$. Then, a basis is given by $\left\{p_{1}, \ldots, p_{n-1}, p_{n}^{\prime}\right\}$, (the rank of $\mathfrak{s o}(2 n, \mathbb{C})$ is $n)$, and the corresponding Hitchin map

$$
p: \mathcal{M} \rightarrow \bigoplus_{i=1}^{n-1} H^{0}\left(K^{2 i}\right) \oplus H^{0}\left(K^{n}\right)
$$

is defined by

$$
p(A, \varphi)=\left(p_{1}(\varphi), \ldots, p_{n-1}(\varphi), p_{n}^{\prime}(\varphi)\right) .
$$

The nilpotent regular element is now

$$
e=\sum_{\alpha \in \Delta^{+}} c_{\alpha} X_{\alpha} \in \mathfrak{s o}(2 n, \mathbb{C}) \text { with } c_{\alpha} \neq 0 \text { for } \alpha \in \Pi
$$

where

$$
\Delta^{+}=\left\{e_{i} \pm e_{j} \text { with } 1 \leq i<j \leq n\right\}
$$




$$
\Pi=\left\{\alpha_{i}=e_{i}-e_{i+1}(1 \leq i \leq n-1), \alpha_{n}=e_{n-1}+e_{n}\right\}
$$

and the corresponding root vectors are

$$
X_{e_{i}-e_{j}}=E_{i j}-E_{n+j, n+i} \text { and } X_{e_{i}+e_{j}}=E_{i, n+j}-E_{j, n+i}
$$

(We are using the same notations as in Section 2.1).

The element $x$, that we can consider in the Cartan subalgebra, is of the form $x=$ $\sum_{i=1}^{n} h_{i}\left(E_{i, i}-E_{n+i, n+i}\right)$. Imposing $[x, e]=2 e$ we obtain that

$$
x=\sum_{i=1}^{n} 2(n-i)\left(E_{i, i}-E_{n+i, n+i}\right)
$$

and

$$
e=\sum_{\alpha \in \Pi} c_{\alpha} X_{\alpha}
$$

Then, we can take for example $e=\sum_{\alpha \in \Pi} X_{\alpha}$. Finally, the conditions $[x, \tilde{e}]=-2 \tilde{e}$ and $[e, \tilde{e}]=x$ determine $\tilde{e}$.

The adjoint action

$$
\langle x, e, \tilde{e}\rangle \cong \mathfrak{s l}(2, \mathbb{C}) \rightarrow \operatorname{End}(\mathfrak{s o}(2 n, \mathbb{C}))
$$

gives the decomposition

$$
\mathfrak{s o}(2 n, \mathbb{C})=\bigoplus_{i=1}^{n-1} V_{i} \oplus V_{n}
$$

with $\operatorname{dim} V_{i}=4 i-1$, for $1 \leq i \leq n-1$ (the exponents in this case are $m_{i}=2 i-1$ ) and $\operatorname{dim} V_{n}=2 n-1\left(m_{n}=n-1\right)$. That is, for $1 \leq i \leq n-1, V_{i}=S^{4 i-2} \mathbb{C}^{2}$ with eigenvalues $4 i-2,4 i-4, \ldots,-4 i+4,-4 i+2$ for the action of ad $x$ and $V_{n}=S^{2 n-2} \mathbb{C}$ with eigenvalues $2 n-2,2 n-4, \ldots,-2 n+4,-2 n+2$.

The highest weight vectors in this case are $e_{1}, \ldots, e_{n-1}, e_{n}$, where $e_{i}$ has eigenvalue $4 i-2$ for $1 \leq i \leq n-1$, and $e_{n}$ has eigenvalue $2 n-2$. We take $V_{1}=\langle x, e, \tilde{e}\rangle$ and $e=e_{1}$.

Given $\left(\alpha_{1}, \ldots, \alpha_{n-1}, \alpha_{n}\right) \in \bigoplus_{i=1}^{n-1} H^{0}\left(K^{2 i}\right) \oplus H^{0}\left(K^{n}\right)$, the Higgs field in the Hitchin component is the $\operatorname{sum} \varphi=\tilde{e}+\alpha_{1} e+\ldots+\alpha_{n-1} e_{n-1}+\alpha_{n} e_{n}$.

Consider now the representation

$$
\mathfrak{s l}(2, \mathbb{C}) \rightarrow \mathfrak{s o}(2 n, \mathbb{C})=\Lambda^{2}(R)
$$


We know that $\mathfrak{s o}(2 n, \mathbb{C})=S^{2} \mathbb{C}^{2}+S^{6} \mathbb{C}^{2}+\ldots+S^{4 n-6} \mathbb{C}^{2}+S^{2 n-2} \mathbb{C}^{2}=\Lambda^{2}(R)$. Then, $R=S^{2 n-2} \mathbb{C}^{2}+1$, where 1 denotes the trivial representation,

$$
\begin{gathered}
\Lambda^{2}\left(S^{2 n-2} \mathbb{C}^{2}+1\right)=\Lambda^{1}\left(S^{2 n-2}\right) \otimes \Lambda^{1}(1)+\Lambda^{2}\left(S^{2 n-2}\right) \otimes \Lambda^{0}(1)+\Lambda^{0}\left(S^{2 n-2}\right) \otimes \Lambda^{2}(1)= \\
=S^{2 n-2}+\Lambda^{2}\left(S^{2 n-2}\right) .
\end{gathered}
$$

Hence, the vector bundle in the Hitchin component is

$$
\begin{aligned}
\mathbb{E} & =\left(S^{2 n-2}+1\right)\left(K^{1 / 2} \oplus K^{-1 / 2}\right)= \\
& =K^{n-1} \oplus K^{n-2} \oplus \ldots \oplus K \oplus \mathcal{O} \oplus K^{-1} \oplus \ldots \oplus K^{-n+2} \oplus K^{-n+1} \oplus \mathcal{O}_{1}=V \oplus W
\end{aligned}
$$

where the subindex 1 is only to distinguish one trivial bundle from the other, in which we can consider the orthogonal structure given by

$$
Q=\left(\begin{array}{lll|l} 
& & 1 & \\
& \cdot & & \\
1 & & & \\
& & & 1
\end{array}\right)
$$

Since we are considering the algebra $\mathfrak{s o}(2 n, \mathbb{C})$ defined by the orthogonal structure $Q=\left(\begin{array}{cc}0 & I_{n} \\ I_{n} & 0\end{array}\right)$, the Higgs field $\varphi=\tilde{e}+\alpha_{1} e+\ldots+\alpha_{n-1} e_{n-1}+\alpha_{n} e_{n}$ is defined in the following order of the subbundles:

$$
\begin{gathered}
K^{n-1} \oplus K^{n-2} \oplus \ldots \oplus K \oplus \mathcal{O} \oplus K^{-n+1} \oplus K^{-n+2} \oplus \ldots \oplus K^{-1} \oplus \mathcal{O}_{1}, \\
\downarrow \\
K^{n-1} \oplus K^{n-2} \oplus \ldots \oplus K \oplus \mathcal{O}_{1} \oplus K^{-n+1} \oplus K^{-n+2} \oplus \ldots \oplus K^{-1} \oplus \mathcal{O},
\end{gathered}
$$

(or the other way around, changing $\mathcal{O} \leftrightarrow \mathcal{O}_{1}$ ).

The minimum in the Hitchin component is given by the values $\alpha_{1}=\ldots=\alpha_{n}=0$, that is, the minimum is the pair

$$
\left(K^{n-1} \oplus K^{n-2} \oplus \ldots \oplus K \oplus \mathcal{O} \oplus K^{-n+1} \oplus K^{-n+2} \oplus \ldots \oplus K^{-1} \oplus \mathcal{O}_{1}, \varphi=\tilde{e}\right),
$$

which is of the form

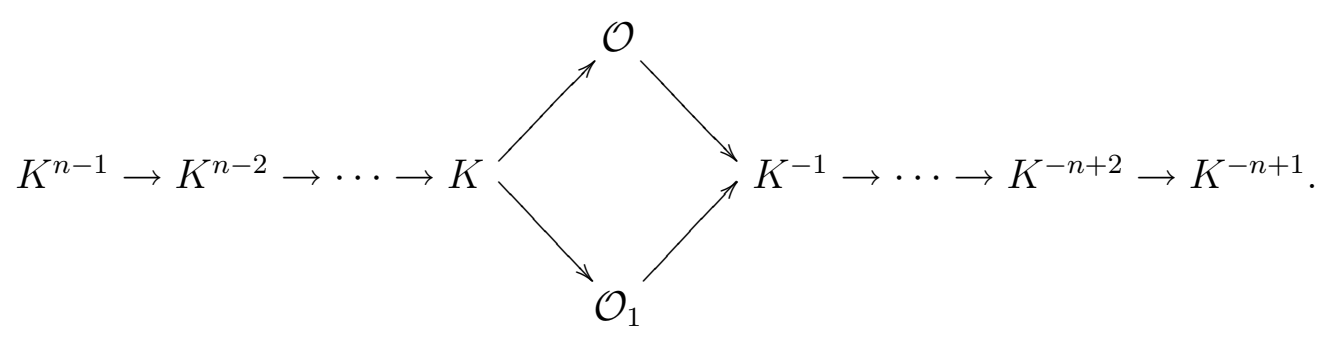


Note that in these diagrams the maps are twisted by $K$ and $K^{m} \rightarrow K^{m-1}$ must be interpreted as $K^{m} \rightarrow K^{m-1} \otimes K=K^{m}$.

To conclude we have to distinguish which powers $K^{i}$ are in $V$ and which in $W$. Observe that the Higgs field alternates $V$ and $W$.

Take $v$ and $v_{1}$ generating $\mathcal{O}$ and $\mathcal{O}_{1}$ respectively. When $n$ is odd, we have $\varphi\left(v-v_{1}\right)=0$. Hence, part of $\mathcal{O}+\mathcal{O}_{1}$ is in $V$ and other in $W$, that is

$$
V_{0}=\left\langle v+v_{1}\right\rangle \text { and } W_{0}=\left\langle v-v_{1}\right\rangle
$$

Then, the minimum in the Hitchin component is

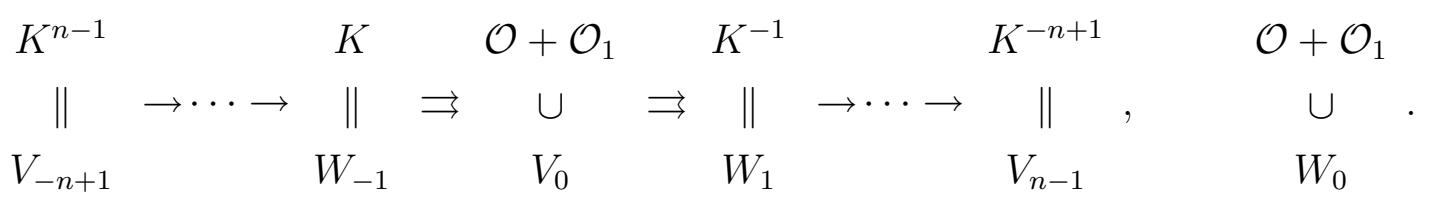

Observe that the situation obtained by interchanging $V$ and $W$ is also possible. This Higgs bundle is a minimum of the type (5) of Theorem 6.10 with $n=\operatorname{rk}\left(W_{0}\right)=1, p=n$ and $V_{-n+1} \cong K^{n-1}$, that is, $\operatorname{deg}\left(V_{-n+1}\right)=(n-1)(2 g-2)$.

When $n$ is even, the sum $\mathcal{O}+\mathcal{O}_{1}$ is entirely contained in $V$ or in $W$, and the minimum in the Hitchin component is

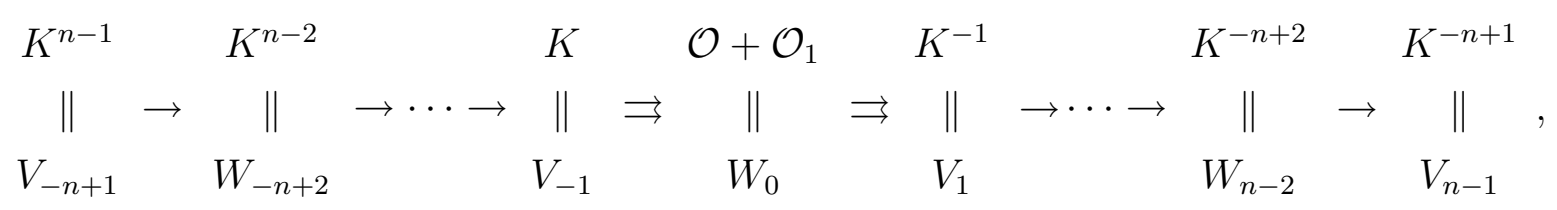

or of the one obtained by interchanging $V$ and $W$. This Higgs bundle is a minimum of the type (3) of Theorem 6.10 with $n=\operatorname{rk}\left(W_{0}\right)=2, p=n$ and $V_{-n+1} \cong K^{n-1}$, that is, $\operatorname{deg}\left(V_{-n+1}\right)=(n-1)(2 g-2)$.

Example 8.3. Hitchin component of the moduli space of polystable $\mathrm{SO}_{0}(3,3)$-Higgs bundles.

The sets of positive and simple roots of $\mathfrak{s o}(6, \mathbb{C})$ are

$$
\begin{gathered}
\Delta^{+}=\left\{e_{1}-e_{2}, e_{2}-e_{3}, e_{1}-e_{3}, e_{1}+e_{2}, e_{2}+e_{3}, e_{1}+e_{3}\right\}, \\
\Pi=\left\{e_{1}-e_{2}, e_{2}-e_{3}, e_{2}+e_{3}\right\} .
\end{gathered}
$$


We know that $e=\left(\begin{array}{rr|lll}c_{e_{1}-e_{2}} & c_{e_{1}-e_{3}} & & c_{e_{1}+e_{2}} & c_{e_{1}+e_{3}} \\ & c_{e_{2}-e_{3}} & -c_{e_{1}+e_{2}} & & c_{e_{2}+e_{3}} \\ & & -c_{e_{1}+e_{3}} & -c_{e_{2}+e_{3}} \\ \hline & & & \\ \hline & & -c_{e_{1}-e_{2}} & \\ -c_{e_{1}-e_{3}} & -c_{e_{2}-e_{3}}\end{array}\right)$, with $c_{e_{1}-e_{2}}, c_{e_{2}-e_{3}}$, $c_{e_{2}+e_{3}} \neq 0$, is a regular nilpotent element. If $x=\left(\begin{array}{llllll}h_{1} & & & & & \\ & h_{2} & & & \\ & & & \\ & & & \\ & & -h_{1} & & \\ & & -h_{2} & \\ & & & -h_{3}\end{array}\right)$ is in the Cartan subalgebra, it satisfies $[x, e]=2 e$ if and only if $h_{1}=4, h_{2}=2$ and $h_{3}=c_{e_{1}-e_{3}}=c_{e_{1}+e_{2}}=c_{e_{1}+e_{3}}=0$. So finally we can consider

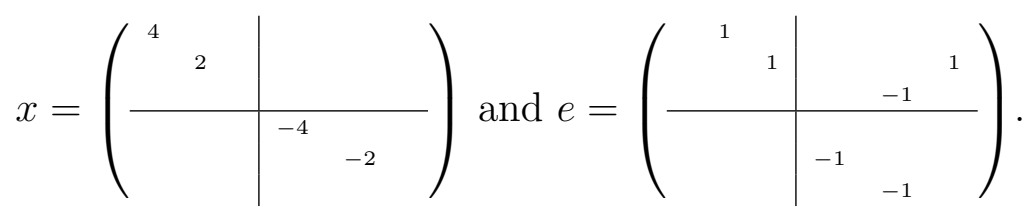

We know that the conditions $[x, \tilde{e}]=-2 \tilde{e}$ and $[e, \tilde{e}]=x$ determine $\tilde{e}$. If

$$
X=\left(\begin{array}{ccc|ccc}
x_{1,1} & x_{1,2} & x_{1,3} & 0 & x_{1,5} & x_{1,6} \\
x_{2,1} & x_{2,2} & x_{2,3} & -x_{1,5} & 0 & x_{2,6} \\
x_{3,1} & x_{3,2} & x_{3,3} & -x_{1,6} & -x_{2,6} & 0 \\
\hline 0 & x_{4,2} & x_{4,3} & -x_{1,1} & -x_{2,1} & -x_{3,1} \\
-x_{4,2} & 0 & x_{5,3} & -x_{1,2} & -x_{2,2} & -x_{3,2} \\
-x_{4,3} & -x_{5,3} & 0 & -x_{1,3} & -x_{2,3} & -x_{3,3}
\end{array}\right) \in \mathfrak{s o}(6, \mathbb{C})
$$

the adjoint action of the semisimple element $x$ on $X$ is given by

$$
\operatorname{ad}(x)(X)=\left(\begin{array}{ccc|ccc}
0 & 2 x_{1,2} & 4 x_{1,3} & 0 & 6 x_{1,5} & 4 x_{1,6} \\
-2 x_{2,1} & 0 & 2 x_{2,3} & -6 x_{1,5} & 0 & 2 x_{2,6} \\
-4 x_{3,1} & -2 x_{3,2} & 0 & -4 x_{1,6} & -2 x_{2,6} & 0 \\
\hline 0 & -6 x_{4,2} & -4 x_{4,3} & 0 & 2 x_{2,1} & 4 x_{3,1} \\
6 x_{4,2} & 0 & -2 x_{5,3} & -2 x_{1,2} & 0 & 2 x_{3,2} \\
4 x_{4,3} & 2 x_{5,3} & 0 & -4 x_{1,3} & -2 x_{2,3} & 0
\end{array}\right),
$$

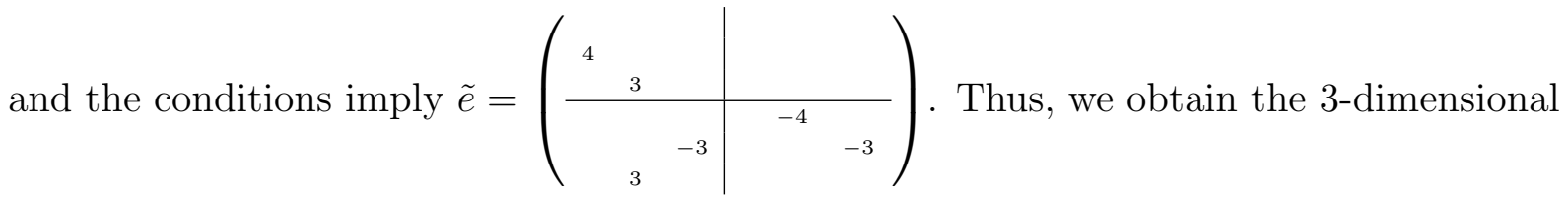


subalgebra

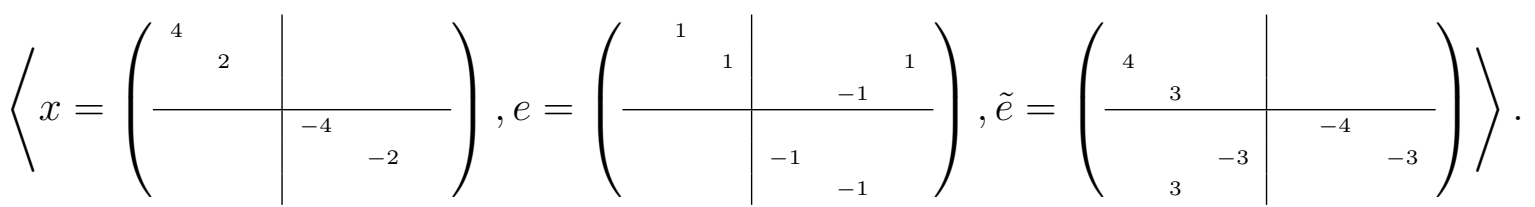

We have a basis of invariant polynomials $\left\{p_{1}, p_{2}, p_{3}\right\}$ of degrees 2,4 and 3 respectively and the decomposition

$$
\mathfrak{s o}(6, \mathbb{C})=V_{1} \oplus V_{2} \oplus V_{3}=S^{2} \mathbb{C}^{2} \oplus S^{6} \mathbb{C}^{2} \oplus S^{4} \mathbb{C}^{2}
$$

of the Lie algebra $\mathfrak{s o}(6, \mathbb{C})$ as a sum of irreducible representations of $\mathfrak{s l}(2, \mathbb{C})$. The highest weight vector of $V_{3}$ is of the form $e_{3}=\left(\begin{array}{l|l}x_{1,3} & x_{1,6} \\ \hline & -x_{1,6} \\ -x_{1,3}\end{array}\right)$ and it is in the kernel of $\operatorname{ad}(e)$ if and only if $x_{1,6}=-x_{1,3}$, then we can take $e_{3}=\left(\begin{array}{l|ll}1 & & -1 \\ & 1 & \\ \hline & & \end{array}\right.$

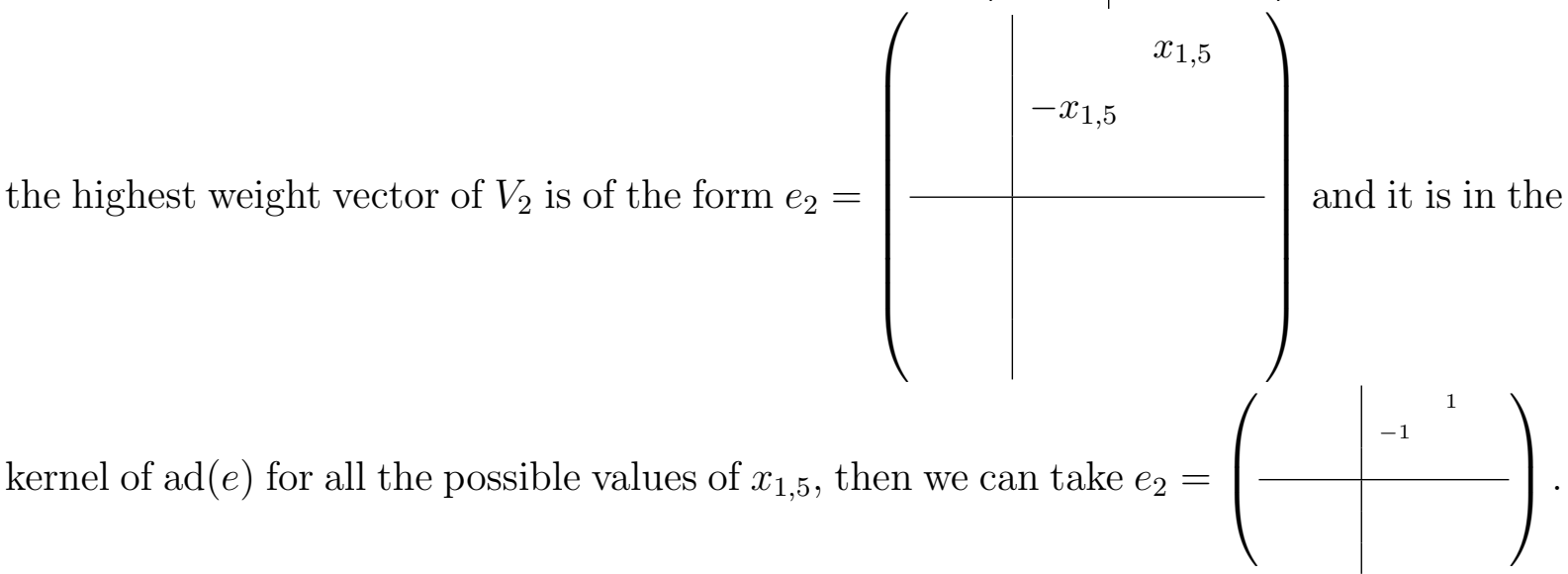
Finally, we define the Higgs field in the Hitchin component by

$$
\varphi=\left(\begin{array}{ccc|ccc} 
& \alpha_{1} & \alpha_{3} & & \alpha_{2} & -\alpha_{3} \\
4 & & \alpha_{1} & -\alpha_{2} & & \alpha_{1} \\
3 & & \alpha_{3} & -\alpha_{1} & \\
\hline & & & -4 & \\
& & -3 & -\alpha_{1} & & -3 \\
& & -\alpha_{3} & -\alpha_{1} &
\end{array}\right)
$$


To obtain the vector bundle in the Hitchin component we need to understand the representation

$$
\mathfrak{s l}(2, \mathbb{C}) \rightarrow \mathfrak{s o}(6, \mathbb{C})=\Lambda^{2}(R) .
$$

We know that $\mathfrak{s o}(6, \mathbb{C})=S^{2} \mathbb{C}^{2}+S^{4} \mathbb{C}^{2}+S^{6} \mathbb{C}^{2}=\Lambda^{2}(R)$. Then, $R=S^{4} \mathbb{C}^{2}+1$. Hence, the vector bundle is

$$
\mathbb{E}=\left(S^{4}+1\right)\left(K^{1 / 2} \oplus K^{-1 / 2}\right)=K^{2} \oplus K \oplus \mathcal{O} \oplus K^{-1} \oplus K^{-2} \oplus \mathcal{O}_{1}=V \oplus W,
$$

and the Higgs field is defined using the following order of the subbundles,

$$
K^{2} \oplus K \oplus \mathcal{O} \oplus K^{-2} \oplus K^{-1} \oplus \mathcal{O}_{1} \longrightarrow K^{2} \oplus K \oplus \mathcal{O}_{1} \oplus K^{-2} \oplus K^{-1} \oplus \mathcal{O}
$$

The minimum in the Hitchin component is given by the values $\alpha_{1}=\alpha_{2}=\alpha_{3}=0$. Taking a basis $K^{2} \oplus K \oplus \mathcal{O} \oplus K^{-2} \oplus K^{-1} \oplus \mathcal{O}_{1}=\left\langle e_{2}, e_{1}, v, e_{-2}, e_{-1}, v_{1}\right\rangle$, we have

$$
\begin{aligned}
& \varphi\left(e_{2}\right)=4 e_{1}, \\
& \varphi\left(e_{1}\right)=3\left(v+v_{1}\right) \\
& \varphi(v)=-3 e_{-1}, \\
& \varphi\left(e_{-2}\right)=0 \\
& \varphi\left(e_{-1}\right)=-4 e_{-2}, \\
& \varphi\left(v_{1}\right)=-3 e_{-1},
\end{aligned}
$$

that is,

$$
e_{2} \stackrel{4}{\longrightarrow} e_{1} \stackrel{3}{\longrightarrow} v+v_{1} \stackrel{-3}{\longrightarrow} e_{-1} \stackrel{-4}{\longrightarrow} e_{-2},
$$

and $\varphi\left(v-v_{1}\right)=0$. If we denote $V_{0}=\left\langle v+v_{1}\right\rangle$ and $W_{0}=\left\langle v-v_{1}\right\rangle$, the minimum in the Hitchin component is

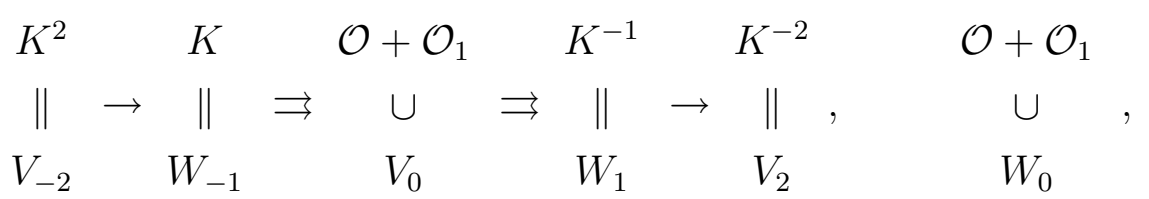

which is a minimum of the type (5) of Theorem 6.10 with $n=\operatorname{rk}\left(W_{0}\right)=1$ and $p=q=3$. Example 8.4. Hitchin component of the moduli space of polystable $\mathrm{SO}_{0}(4,4)$-Higgs bundles.

The sets of positive and simple roots of $\mathfrak{s o}(8, \mathbb{C})$ are

$$
\begin{gathered}
\Delta^{+}=\left\{e_{1}-e_{2}, e_{1}-e_{3}, e_{1}-e_{4}, e_{2}-e_{3}, e_{2}-e_{4}, e_{3}-e_{4}, e_{1}+e_{2}, e_{1}+e_{3}, e_{1}+e_{4}, e_{2}+e_{3}, e_{2}+e_{4}, e_{3}+e_{4},\right\} \\
\Pi=\left\{e_{1}-e_{2}, e_{2}-e_{3}, e_{3}-e_{4}, e_{3}+e_{4}\right\}
\end{gathered}
$$


Using the same process as in the previous example, we obtain the 3-dimensional subalgebra
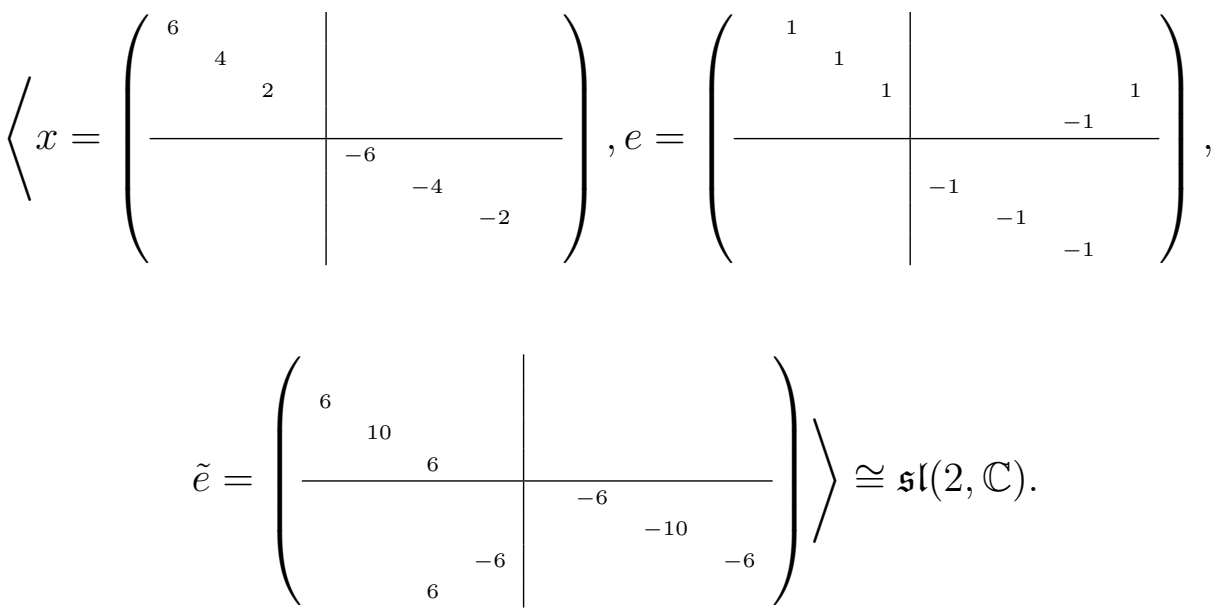

$$
\text { If } X=\left(\begin{array}{cccc|cccc}
x_{1,1} & x_{1,2} & x_{1,3} & x_{1,4} & 0 & x_{1,6} & x_{1,7} & x_{1,8} \\
x_{2,1} & x_{2,2} & x_{2,3} & x_{2,4} & -x_{1,6} & 0 & x_{2,7} & x_{2,8} \\
x_{3,1} & x_{3,2} & x_{3,3} & x_{3,4} & -x_{1,7} & -x_{2,7} & 0 & x_{3,8} \\
x_{4,1} & x_{4,2} & x_{4,3} & x_{4,4} & -x_{1,8} & -x_{2,8} & -x_{3,8} & 0 \\
\hline 0 & x_{5,2} & x_{5,3} & x_{5,4} & -x_{1,1} & -x_{2,1} & -x_{3,1} & -x_{4,1} \\
-x_{5,2} & 0 & x_{6,3} & x_{6,4} & -x_{1,2} & -x_{2,2} & -x_{3,2} & -x_{4,2} \\
-x_{5,3} & -x_{6,3} & 0 & x_{7,4} & -x_{1,3} & -x_{2,3} & -x_{3,3} & -x_{4,3} \\
-x_{5,4} & -x_{6,4} & -x_{7,4} & 0 & -x_{1,4} & -x_{2,4} & -x_{3,4} & -x_{4,4}
\end{array}\right) \in \mathfrak{s o}(8, \mathbb{C}),
$$

the adjoint action of the semisimple element $x$ on $X$ is given by

$$
\operatorname{ad}(x)(X)=\left(\begin{array}{cccc|cccc}
0 & 2 x_{1,2} & 4 x_{1,3} & 6 x_{1,4} & 0 & 10 x_{1,6} & 8 x_{1,7} & 6 x_{1,8} \\
-2 x_{2,1} & 0 & 2 x_{2,3} & 4 x_{2,4} & -10 x_{1,6} & 0 & 6 x_{2,7} & 4 x_{2,8} \\
-4 x_{3,1} & -2 x_{3,2} & 0 & 2 x_{3,4} & -8 x_{1,7} & -6 x_{2,7} & 0 & 2 x_{3,8} \\
-6 x_{4,1} & -4 x_{4,2} & -2 x_{4,3} & 0 & -6 x_{1,8} & -4 x_{2,8} & -2 x_{3,8} & 0 \\
\hline 0 & -10 x_{5,2} & -8 x_{5,3} & -6 x_{5,4} & 0 & 2 x_{2,1} & 4 x_{3,1} & 6 x_{4,1} \\
10 x_{5,2} & 0 & -6 x_{6,3} & -4 x_{6,4} & -2 x_{1,2} & 0 & 2 x_{3,2} & 4 x_{4,2} \\
8 x_{5,3} & 6 x_{6,3} & 0 & -2 x_{7,4} & -4 x_{1,3} & -2 x_{2,3} & 0 & 2 x_{4,3} \\
6 x_{5,4} & 4 x_{6,4} & 2 x_{7,4} & 0 & -6 x_{1,4} & -4 x_{2,4} & -2 x_{3,4} & 0
\end{array}\right) .
$$

We take a basis of the algebra of invariant polynomials $\left\{p_{1}, p_{2}, p_{3}, p_{4}\right\}$ of degrees $2,4,6$ and 4 respectively. We also have a decomposition

$$
\mathfrak{s o}(8, \mathbb{C})=V_{1} \oplus V_{2} \oplus V_{3} \oplus V_{4}=S^{2} \mathbb{C}^{2} \oplus S^{6} \mathbb{C}^{2} \oplus S^{10} \mathbb{C}^{2} \oplus S^{6} \mathbb{C}^{2},
$$

of the Lie algebra $\mathfrak{s o}(8, \mathbb{C})$ as a sum of irreducible representations of $\mathfrak{s l}(2, \mathbb{C})$. The highest 
weight vector of $V_{3}$ is of the form $e_{3}=\left(\begin{array}{c|c}x_{1,6} \\ \text { of } \operatorname{ad}(e) \text { for all the possible values of } x_{1,6} \text {, then we can take } e_{3}=(-1\end{array}\right)$ and it is in the kernel

An eigenvector with eigenvalue 6 has to be of the form:

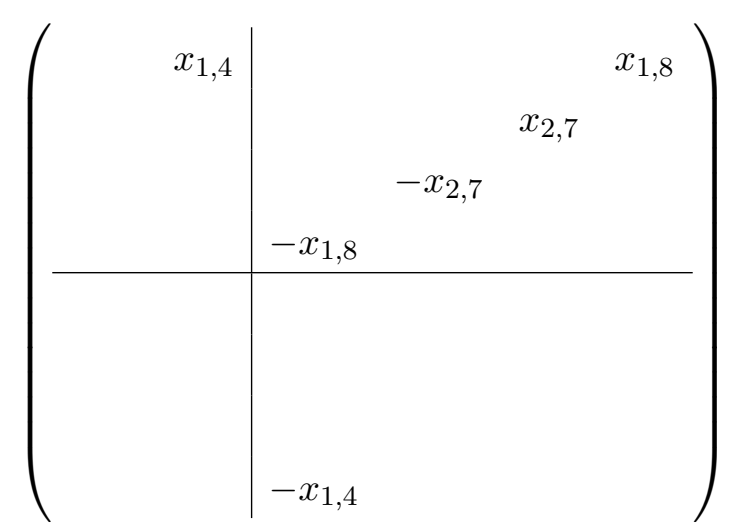

and it is in the kernel of ad(e) if and only if $x_{1,4}+x_{1,8}+x_{2,7}=0$. Then we can take, for example,

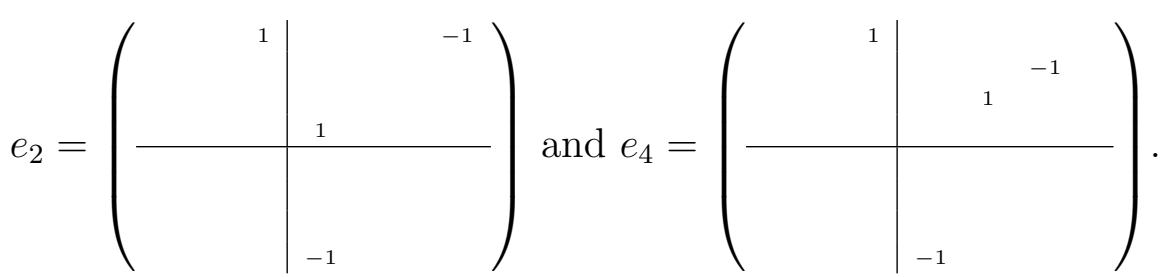

We define the Higgs field in the Hitchin component by

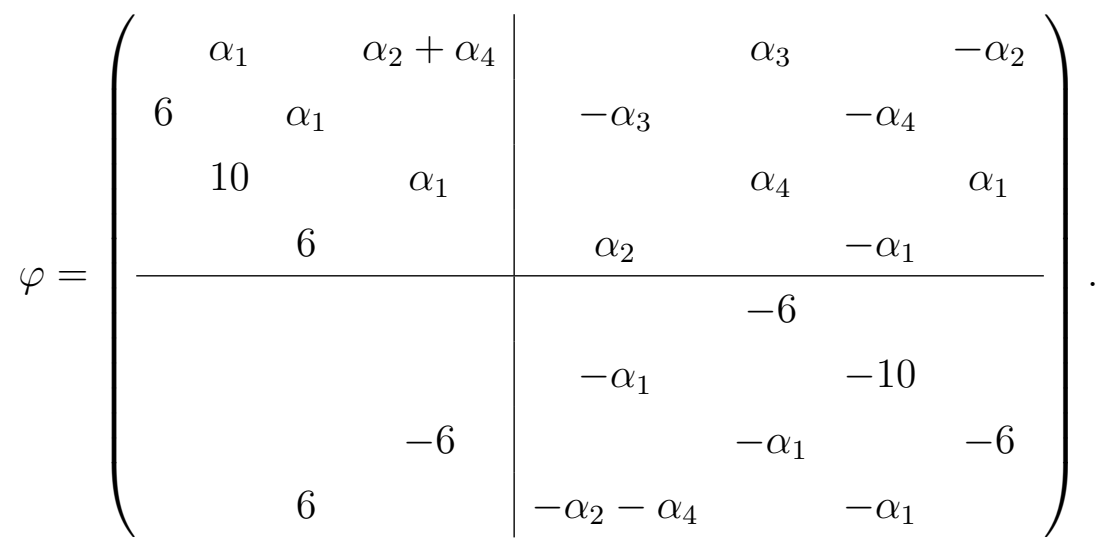


To obtain the vector bundle in the Hitchin component we have to understand the representation

$$
\mathfrak{s l}(2, \mathbb{C}) \rightarrow \mathfrak{s o}(8, \mathbb{C})=\Lambda^{2}(R)
$$

We know that $\mathfrak{s o}(8, \mathbb{C})=S^{2} \mathbb{C}^{2} \oplus S^{6} \mathbb{C}^{2} \oplus S^{10} \mathbb{C}^{2} \oplus S^{6} \mathbb{C}^{2}=\Lambda^{2}(R)$. Then, $R=S^{6} \mathbb{C}^{2}+1$. Hence, the vector bundle is

$$
\mathbb{E}=\left(S^{6}+1\right)\left(K^{1 / 2} \oplus K^{-1 / 2}\right)=K^{3} \oplus K^{2} \oplus K \oplus \mathcal{O} \oplus K^{-1} \oplus K^{-2} \oplus K^{-3} \oplus \mathcal{O}_{1}=V \oplus W
$$

and the Higgs field is defined using the following order of the subbundles,

$$
\begin{array}{r}
K^{3} \oplus K^{2} \oplus K \oplus \mathcal{O} \oplus K^{-3} \oplus K^{-2} \oplus K^{-1} \oplus \mathcal{O}_{1} \\
\downarrow \\
K^{3} \oplus K^{2} \oplus K \oplus \mathcal{O}_{1} \oplus K^{-3} \oplus K^{-2} \oplus K^{-1} \oplus \mathcal{O} .
\end{array}
$$

The minimum is given by the values $\alpha_{1}=\alpha_{2}=\alpha_{3}=\alpha_{4}=0$. Taking a basis $K^{3} \oplus K^{2} \oplus K \oplus \mathcal{O} \oplus K^{-3} \oplus K^{-2} \oplus K^{-1} \oplus \mathcal{O}_{1}=\left\langle e_{3}, e_{2}, e_{1}, v, e_{-3}, e_{-2}, e_{-1}, v_{1}\right\rangle$, we have

$$
\begin{aligned}
\varphi\left(e_{3}\right) & =6 e_{2}, \\
\varphi\left(e_{2}\right) & =10 e_{1} \\
\varphi\left(e_{1}\right) & =6\left(v+v_{1}\right) \\
\varphi(v) & =-6 e_{-1}, \\
\varphi\left(e_{-3}\right) & =0 \\
\varphi\left(e_{-2}\right) & =-6 e_{-3}, \\
\varphi\left(e_{-1}\right) & =-10 e_{-2}, \\
\varphi\left(v_{1}\right) & =-6 e_{-1},
\end{aligned}
$$

that is,

$$
e_{3} \stackrel{6}{\longrightarrow} e_{2} \stackrel{10}{\longrightarrow} e_{1} \stackrel{6}{\longrightarrow} v, v_{1} \stackrel{-6}{\longrightarrow} e_{-1} \stackrel{-10}{\longrightarrow} e_{-2} \stackrel{-6}{\longrightarrow} e_{-3} .
$$

Then, we take $V_{0}=0$ and $W_{0}=\left\langle v, v_{1}\right\rangle=\mathcal{O}+\mathcal{O}_{1}$, and the minimum in the Hitchin component is

$$
\begin{aligned}
& \begin{array}{llllll}
K^{3} & K^{2} & K & \mathcal{O}+\mathcal{O}_{1} & K^{-1} & K^{-2}
\end{array} K^{-3} \\
& \|\rightarrow\| \rightarrow\|\rightrightarrows\| \quad \rightarrow \quad \rightarrow\|\rightarrow\| \quad \\
& \begin{array}{lllllll}
V_{-3} & W_{-2} & V_{-1} & W_{0} & V_{1} & W_{2} & V_{3}
\end{array}
\end{aligned}
$$

which is a minimum of the type (3) of Theorem 6.10 with $n=\operatorname{rk}\left(W_{0}\right)=2$ and $p=q=4$. 


\subsection{Hitchin component for $\mathrm{SO}_{0}(n, n+1)$}

The Lie group $\mathrm{SO}_{0}(n, n+1)$ is the split real form of $\mathrm{SO}(2 n+1, \mathbb{C})$. A basis of the invariant polynomials on $\mathfrak{s o}(2 n+1, \mathbb{C})$ is provided by the coefficients $\left\{p_{1}, \ldots, p_{n-1}, p_{n}\right\}$ of the characteristic polynomial

$$
\operatorname{det}(x-A)=x\left(x^{2 n}+p_{1}(A) x^{2 n-2}+\ldots+p_{n}(A)\right),
$$

where $\operatorname{deg} p_{i}=2 i($ the rank of $\mathfrak{s o}(2 n+1, \mathbb{C})$ is $n)$. The corresponding Hitchin map

$$
p: \mathcal{M} \rightarrow \bigoplus_{i=1}^{n} H^{0}\left(K^{2 i}\right)
$$

is defined by

$$
p(A, \varphi)=\left(p_{1}(\varphi), \ldots, p_{n}(\varphi)\right)
$$

The nilpotent regular element is now

$$
e=\sum_{\alpha \in \Delta^{+}} c_{\alpha} X_{\alpha} \in \mathfrak{s o}(2 n+1, \mathbb{C}) \text { with } c_{\alpha} \neq 0 \text { for } \alpha \in \Pi,
$$

where

$$
\begin{gathered}
\Delta^{+}=\left\{e_{i} \pm e_{j} \text { with } 1 \leq i<j \leq n\right\} \cup\left\{e_{i} \text { with } 1 \leq i \leq n\right\}, \\
\Pi=\left\{\alpha_{i}=e_{i}-e_{i+1}(1 \leq i \leq n-1), \alpha_{n}=e_{n}\right\},
\end{gathered}
$$

and the corresponding root vectors are

$$
\begin{aligned}
X_{e_{i}-e_{j}} & =E_{i j}-E_{n+j, n+i}, & X_{e_{i}+e_{j}} & =E_{i, n+j}-E_{j, n+i}, \\
X_{e_{i}} & =E_{i, 2 n+1}-E_{2 n+1, n+i}, & X_{-e_{i}} & =E_{n+i, 2 n+1}-E_{2 n+1, i} .
\end{aligned}
$$

The element $x$, that we can consider in the Cartan subalgebra, is of the form $x=$ $\sum_{i=1}^{n} h_{i}\left(E_{i, i}-E_{n+i, n+i}\right)$. Imposing $[x, e]=2 e$ we obtain that

$$
x=\sum_{i=1}^{n} 2(n+1-i)\left(E_{i, i}-E_{n+i, n+i}\right)
$$

and

$$
e=\sum_{\alpha \in \Pi} c_{\alpha} X_{\alpha}
$$

We take $e=\sum_{\alpha \in \Pi} X_{\alpha}$. Finally, the conditions $[x, \tilde{e}]=-2 \tilde{e}$ and $[e, \tilde{e}]=x$ determine $\tilde{e}$.

The adjoint action

$$
\langle x, e, \tilde{e}\rangle \cong \mathfrak{s l}(2, \mathbb{C}) \rightarrow \operatorname{End}(\mathfrak{s o}(2 n+1, \mathbb{C})),
$$


gives the decomposition

$$
\mathfrak{s o}(2 n+1, \mathbb{C})=\bigoplus_{i=1}^{n} V_{i}
$$

with $\operatorname{dim} V_{i}=4 i-1$, for $1 \leq i \leq n$ (the exponents in this case are $m_{i}=2 i-1$ ). That is, $V_{i}=S^{4 i-2} \mathbb{C}^{2}, 1 \leq i \leq n$, with eigenvalues $4 i-2,4 i-4, \ldots,-4 i+4,-4 i+2$ for the action of ad $x$.

The highest weight vectors in this case are $e_{1}, \ldots, e_{n-1}, e_{n}$, where $e_{i}$ has eigenvalue $4 i-2$ for $1 \leq i \leq n$. We take $V_{1}=\langle x, e, \tilde{e}\rangle$ and $e=e_{1}$.

Given $\left(\alpha_{1}, \ldots, \alpha_{n}\right) \in \bigoplus_{i=1}^{n} H^{0}\left(K^{2 i}\right)$, the Higgs field in the Hitchin component is the $\operatorname{sum} \varphi=\tilde{e}+\alpha_{1} e+\ldots+\alpha_{n} e_{n}$.

Consider now the representation

$$
\mathfrak{s l}(2, \mathbb{C}) \rightarrow \mathfrak{s o}(2 n+1, \mathbb{C})=\Lambda^{2}(R)
$$

We know that $\mathfrak{s o}(2 n+1, \mathbb{C})=S^{2} \mathbb{C}^{2}+S^{6} \mathbb{C}^{2}+\ldots+S^{4 n-2} \mathbb{C}^{2}=\Lambda^{2}(R)$. Then, $R=S^{2 n} \mathbb{C}^{2}$. Hence, the vector bundle is

$$
\mathbb{E}=S^{2 n}\left(K^{1 / 2} \oplus K^{-1 / 2}\right)=K^{n} \oplus \ldots \oplus K \oplus \mathcal{O} \oplus K^{-1} \oplus \ldots \oplus K^{-n}=V \oplus W,
$$

and the field $\varphi$ is defined in the following order of the subbundles: $K^{n} \oplus K^{n-1} \oplus \ldots \oplus$ $K \oplus K^{-n} \oplus K^{-n+1} \oplus \ldots \oplus K^{-1} \oplus \mathcal{O}$.

The minimum in the Hitchin component corresponds with the values $\alpha_{1}=\ldots=\alpha_{n}=$ 0 and then it is the pair

$$
\left(K^{n} \oplus K^{n-1} \oplus \ldots \oplus K \oplus K^{-n} \oplus K^{-n+1} \oplus \ldots \oplus K^{-1} \oplus \mathcal{O}, \varphi=\tilde{e}\right),
$$

which is of the form

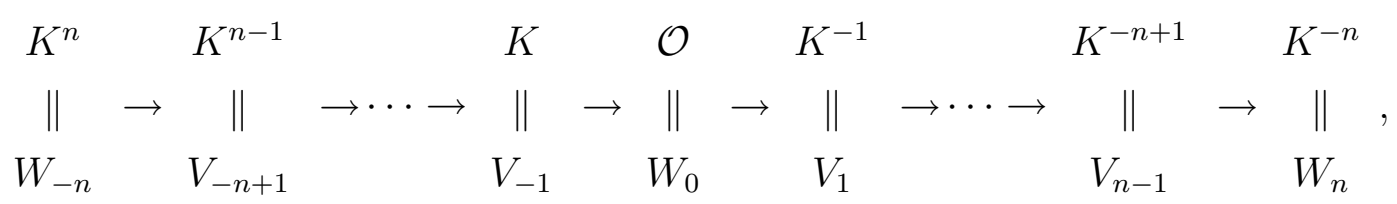

when $n$ is even and of the form

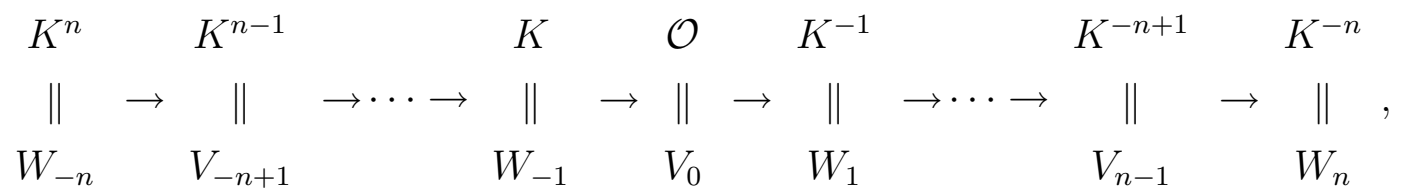

when $n$ is odd. These Higgs bundles are minima of the type (1) and (2) of Theorem 6.10 respectively with $p=n$ and $W_{-n} \cong K^{n}$, that is, $\operatorname{deg}\left(W_{-n}\right)=n(2 g-2)$. 
Example 8.5. Hitchin component of the moduli space of polystable $\mathrm{SO}_{0}(3,4)$-Higgs bundles.

The sets of positive and simple roots of $\mathfrak{s o}(7, \mathbb{C})$ are

$$
\begin{gathered}
\Delta^{+}=\left\{e_{1}-e_{2}, e_{1}-e_{3}, e_{2}-e_{3}, e_{1}+e_{2}, e_{1}+e_{3}, e_{2}+e_{3}, e_{1}, e_{2}, e_{3}\right\}, \\
\Pi=\left\{e_{1}-e_{2}, e_{2}-e_{3}, e_{3}\right\} .
\end{gathered}
$$

Using the same process as in the previous examples, we obtain the 3-dimensional subalgebra
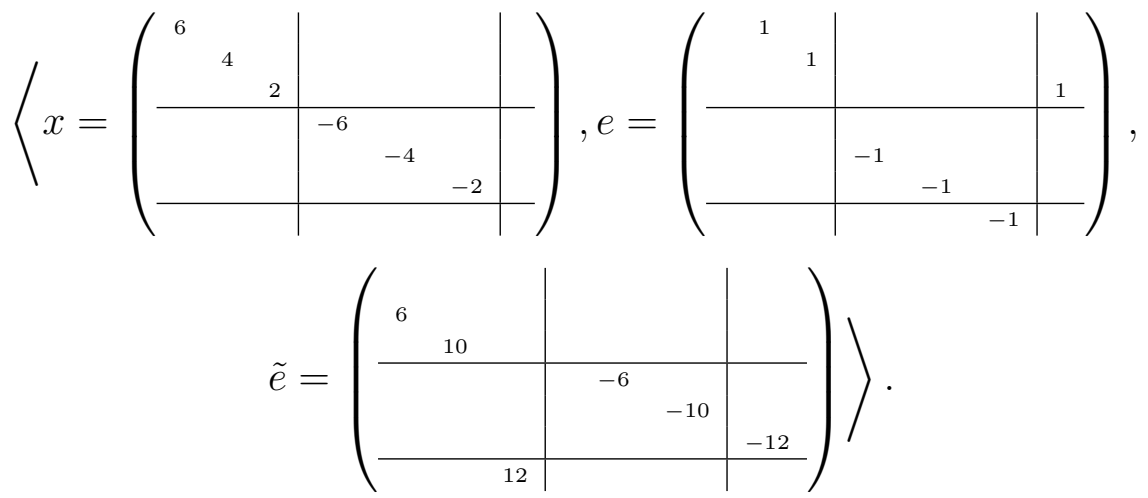

$$
\text { If } X=\left(\begin{array}{ccc|ccc|c}
x_{1,1} & x_{1,2} & x_{1,3} & 0 & x_{1,5} & x_{1,6} & x_{1,7} \\
x_{2,1} & x_{2,2} & x_{2,3} & -x_{1,5} & 0 & x_{2,6} & x_{2,7} \\
x_{3,1} & x_{3,2} & x_{3,3} & -x_{1,6} & -x_{2,6} & 0 & x_{3,7} \\
\hline 0 & x_{4,2} & x_{4,3} & -x_{1,1} & -x_{2,1} & -x_{3,1} & x_{4,7} \\
-x_{4,2} & 0 & x_{5,3} & -x_{1,2} & -x_{2,2} & -x_{3,2} & x_{5,7} \\
-x_{4,3} & -x_{5,3} & 0 & -x_{1,3} & -x_{2,3} & -x_{3,3} & x_{6,7} \\
\hline-x_{4,7} & -x_{5,7} & -x_{6,7} & -x_{1,7} & -x_{2,7} & -x_{3,7} & 0
\end{array}\right) \in \mathfrak{s o}(7, \mathbb{C}) \text {, }
$$

the adjoint action of the semisimple element $x$ on $X$ is given by

$$
\operatorname{ad}(x)(X)=\left(\begin{array}{ccc|ccc|c}
0 & 2 x_{1,2} & 4 x_{1,3} & 0 & 10 x_{1,5} & 8 x_{1,6} & 6 x_{1,7} \\
-2 x_{2,1} & 0 & 2 x_{2,3} & -10 x_{1,5} & 0 & 6 x_{2,6} & 4 x_{2,7} \\
-4 x_{3,1} & -2 x_{3,2} & 0 & -8 x_{1,6} & -6 x_{2,6} & 0 & 2 x_{3,7} \\
\hline 0 & -10 x_{4,2} & -8 x_{4,3} & 0 & 2 x_{2,1} & 4 x_{3,1} & -6 x_{4,7} \\
10 x_{4,2} & 0 & -6 x_{5,3} & -2 x_{1,2} & 0 & 2 x_{3,2} & -4 x_{5,7} \\
8 x_{4,3} & 6 x_{5,3} & 0 & -4 x_{1,3} & -2 x_{2,3} & 0 & -2 x_{6,7} \\
\hline 6 x_{4,7} & 4 x_{5,7} & 2 x_{6,7} & -6 x_{1,7} & -4 x_{2,7} & -2 x_{3,7} & 0
\end{array}\right) .
$$

We take a basis of the algebra of invariant polynomials $\left\{p_{1}, p_{2}, p_{3}\right\}$ with degrees $2,4,6$ respectively. We also have a decomposition

$$
\mathfrak{s o}(7, \mathbb{C})=V_{1} \oplus V_{2} \oplus V_{3}=S^{2} \mathbb{C}^{2} \oplus S^{6} \mathbb{C}^{2} \oplus S^{10} \mathbb{C}^{2}
$$


of the Lie algebra $\mathfrak{s o}(7, \mathbb{C})$ as a sum of irreducible representations of $\mathfrak{s l}(2, \mathbb{C})$. The highest weight vector of $V_{2}$ is of the form $e_{2}=\left(\begin{array}{l|r|r}\mid & x_{2,6} \\ & -x_{2,6} & \\ \hline & & \end{array}\right)$ and it is in the kernel of $\operatorname{ad}(e)$ if and only if $x_{2,6}+x_{1,7}=0$. We can take $e_{2}=\left(\begin{array}{l|l|l|l} & & & \\ & & & \\ & & & \end{array}\right)$.

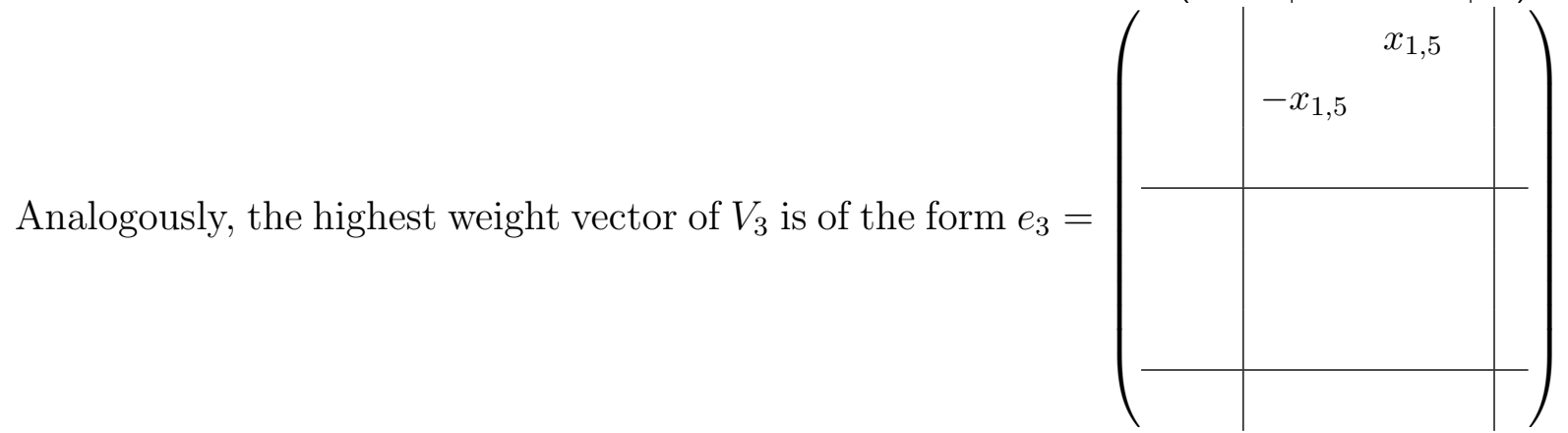
and it is in the kernel of $\operatorname{ad}(e)$ for all the possible values of $x_{1,5}$, then we can take $e_{3}=\left(\begin{array}{l|ll|l} & { }^{1} & 1 & \\ & & & \\ & & & \end{array}\right)$. Finally, we define the Higgs field in the Hitchin component by

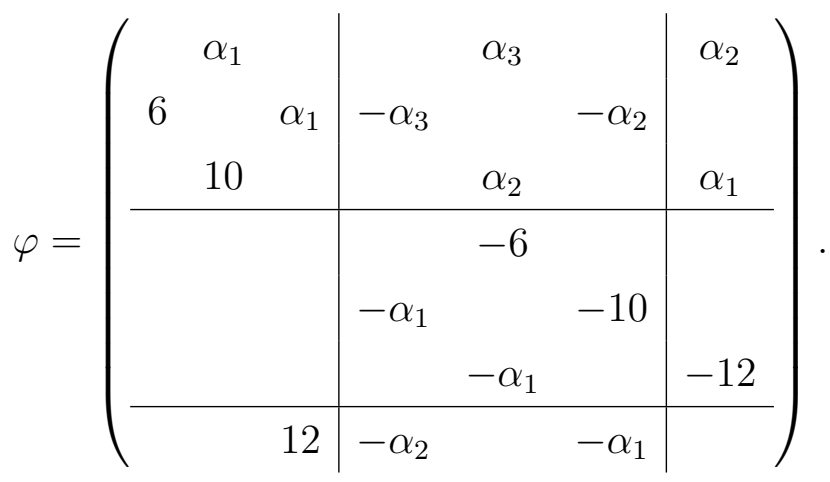

To obtain the vector bundle in the Hitchin component we have to understand the representation

$$
\mathfrak{s l}(2, \mathbb{C}) \rightarrow \mathfrak{s o}(7, \mathbb{C})=\Lambda^{2}(R)
$$

We know that $\mathfrak{s o}(7, \mathbb{C})=S^{2} \mathbb{C}^{2}+S^{6} \mathbb{C}^{2}+S^{10} \mathbb{C}^{2}=\Lambda^{2}(R)$. Then, $R=S^{6} \mathbb{C}^{2}$. Hence, the 
vector bundle is

$$
\mathbb{E}=S^{6}\left(K^{1 / 2} \oplus K^{-1 / 2}\right)=K^{3} \oplus K^{2} \oplus K \oplus \mathcal{O} \oplus K^{-1} \oplus K^{-2} \oplus K^{-3}=V \oplus W,
$$

and the Higgs field $\varphi$ is defined in the following order of the subbundles: $K^{3} \oplus K^{2} \oplus K \oplus$ $K^{-3} \oplus K^{-2} \oplus K^{-1} \oplus \mathcal{O}$.

The minimum in the Hitchin component corresponds with the values $\alpha_{1}=\alpha_{2}=\alpha_{3}=$ 0. Taking the basis $K^{3} \oplus K^{2} \oplus K \oplus K^{-3} \oplus K^{-2} \oplus K^{-1} \oplus \mathcal{O}=\left\langle e_{3}, e_{2}, e_{1}, e_{-3}, e_{-2}, e_{-1}, v\right\rangle$, we have

$$
\begin{aligned}
\varphi\left(e_{3}\right) & =6 e_{2}, \\
\varphi\left(e_{2}\right) & =10 e_{1}, \\
\varphi\left(e_{1}\right) & =12 v, \\
\varphi\left(e_{-3}\right) & =0, \\
\varphi\left(e_{-2}\right) & =-6 e_{-3}, \\
\varphi\left(e_{-1}\right) & =-10 e_{-2}, \\
\varphi(v) & =-12 e_{-1},
\end{aligned}
$$

that is,

$$
e_{3} \stackrel{6}{\longrightarrow} e_{2} \stackrel{10}{\longrightarrow} e_{1} \stackrel{12}{\longrightarrow} v \stackrel{-12}{\longrightarrow} e_{-1} \stackrel{-10}{\longrightarrow} e_{-2} \stackrel{-6}{\longrightarrow} e_{-3} .
$$

Thus, the minimum in the Hitchin component is

$$
\begin{aligned}
& \begin{array}{lllllll}
K^{3} & K^{2} & K & \mathcal{O} & K^{-1} & K^{-2} & K^{-3}
\end{array} \\
& \|\rightarrow\| \rightarrow\|\rightarrow\| \rightarrow\|\rightarrow\|, \\
& \begin{array}{lllllll}
W_{-3} & V_{-2} & W_{-1} & V_{0} & W_{1} & V_{2} & W_{3}
\end{array}
\end{aligned}
$$

which is a minimum of the type (2) of Theorem 6.10 with $p=3$. 


\section{References}

[1] J. F. Adams, Lectures on Exceptional Lie Groups, Chicago Lectures on Mathematics, (1996).

[2] J. Amorós, M. Burger, K. Corlette, D. Kotschick and D. Toledo, Fundamental groups of compact Kähler manifolds, Mathematical Surveys and Monographs, 44 (1996), Amer. Math. Soc.

[3] M. F. Atiyah and R. Bott, The Yang-Mills equations over Riemann surfaces, Philos. Trans. Roy. Soc. London Ser. A 308 (1982), 523-615.

[4] P. Bartolomeis, Principal bundles in action, Riv. Mat. Univ. Parma (4) 17 (1991), $1-65$.

[5] I. Biswas and S. Ramanan, An infinitesimal study of the moduli of Hitchin pairs, J. London Math. Soc. (2) 49 (1994), 219-231.

[6] R. Bott, Lectures on Morse Theory, Old and New, Bull. Amer. Math. Soc. (N.S.) 7 (1982), no. 2, 331-358.

[7] S. B. Bradlow, O. García-Prada and P. B. Gothen, Surface group representations and $\mathrm{U}(p, q)$-Higgs bundles, J. Diff. Geom. 64 (2003), 111-170.

[8] S. B. Bradlow, O. García-Prada and P. B. Gothen, Representations of surface groups in the general linear group, Publications of the RSME, vol. 7 (2004), 89-94.

[9] S. B. Bradlow, O. García-Prada and P. B. Gothen, Maximal surface group representations in isometry groups of classical Hermitian symmetric spaces, Geometriae Dedicata 122 (2006), 185-213.

[10] S. B. Bradlow, O. García-Prada, and I. Mundet i Riera, Relative Hitchin-Kobayashi correspondences for principal pairs, Quart. J. Math. 54 (2003), 171-208.

[11] T. Bröcker and T. tom Dieck, Representations of Compact Lie Groups. GTM 98. Springer-Verlag. (1985).

[12] É. Cartan, Les groupes réeles simples finis et continus, Ann. Sci. École Norm. Sup. 31 (1914), 263-355.

[13] K. Corlette, Flat G-bundles with canonical metrics, J. Diff. Geom. 28 (1988), 361382. 
[14] K. Corlette, Gauge theory and representations of Kahlergroups, Geometry of Group Representations (W. M. Goldman and A. R. Magid, eds.), Contemporary Math., 74 (1988), 107-124.

[15] S. K. Donaldson, Twisted harmonic maps and the self-duality equations, Proc. London Math. Soc. (3) 55 (1987), 127-131.

[16] J Eells, Jr. and J. H. Sampson, Harmonic Mappings of Riemannian Manifolds, American Journal of Mathematics, 86, No. 1 (1964), 109-160.

[17] T. Frankel, Fixed points and torsion on Khler manifolds, Ann. of Math. (2) 70 (1959), $1-8$.

[18] W. Fulton and J. Harris, Representation Theory. A first curse, Springer-Verlag. (1991).

[19] O. García-Prada, P. B. Gothen and I. Mundet i Riera, Representations of surface groups in the real symplectic group, arXiv:0809.0576v2 [math.AG].

[20] O. García-Prada, P. B. Gothen and V. Muñoz, Betti numbers of the moduli space of rank 3 parabolic Higgs bundles, Mem. Amer. Math. Soc. 187 (2007), no. 879, viii+80 pp.

[21] O. García-Prada and I. Mundet i Riera, Representations of the fundamental group of a closed oriented surface in $\operatorname{Sp}(4, \mathbb{R})$, Topology 43 (2004), 831-855.

[22] W. M. Goldman, The symplectic nature of fundamental groups of surfaces, Adv. Math. 54 (1984), No. 2, 200-225.

[23] W. M. Goldman, Topological components of spaces of representations, Invent. Math. 93 (1988), 557-607.

[24] P. B. Gothen, The topology of Higgs bundle moduli spaces, Ph.D. thesis, Mathematics Institute, University of Warwick (1995).

[25] P. B. Gothen, Components of spaces of representations and stable triples, Topology 40 (2001), 823-850.

[26] Ph. Griffiths and J. Harris, Principles of algebraic geometry, John Wiley, New York, (1978).

[27] Brian C. Hall, Lie Groups, Lie Algebras and Representations, Springer-Verlag. (2004). 
[28] F.Reese Harvey, Spinors and Calibrations, Academic Press, Inc. (1990).

[29] S. Helgason, Differential geometry, Lie groups and symmetric spaces, Mathematics, vol. 80, Academic Press, San Diego (1978).

[30] N. J. Hitchin, Stable bundles and integrable systems, Duke Math. J. 54 (1987), no. $1,91-114$.

[31] N. J. Hitchin, The self-duality equations on a Riemann surface, Proc. London Math. Soc. (3) 55 (1987), 59-126.

[32] N. J. Hitchin, Lie groups and Teichmüller space, Topology 31 (1992), 449-473.

[33] G. H. Hitching, A remark on subbundles of symplectic and orthogonal vector bundles over curves, arXiv:math/0407323[math.AG].

[34] J. E. Humphreys, Introduction to Lie Algebras and Representation Theory, SpringerVerlag, New York (1980).

[35] S. Kobayashi, Differential geometry of complex vector bundles, Princeton University Press and Mathematical Society of Japan (1987).

[36] S. Kobayashi and K. Nomizu, Foundations of differential geometry, Volume 1, Interscience Tracts in Pure and Applied Mathematics 15 New York-London, John Wiley and Sons, (1963).

[37] B. Kostant, The principal three-dimensional subgroup and the Betti numbers of a complex simple Lie group, Amer. J. Math. 81 (1959), 973-1032.

[38] B. Kostant, Lie group representations on polynomial rings, Amer. J. Math. 85 (1963), 327-404.

[39] B. Kostant and S. Rallis, Orbits and representations associated with symmetric spaces, Amer. J. Math. 93 (1971), 753-809.

[40] J. W. Milnor, Morse theory, Annals of Mathematics Studies, No. 51, Princeton University Press, Princeton, NJ. (1963).

[41] M. S. Narasimhan, C. S. Seshadri, Stable and unitary vector bundles on a compact Riemann surface, Ann. Math. 82 (1965), 540-567.

[42] N. Nitsure, Moduli spaces of semistable pairs on a curve, Proc. London Math. Soc. 62 (1991), 275-300. 
[43] A. G. Oliveira, Representations of surface groups in the projective general linear group, arXiv:0901.2314[math.AG].

[44] A. L. Onishchik and E. B. Vinverg, Lie Groups and Lie Algebras III. Encyclopedia of Mathematical Science, vol. 41. Springer-Verlag. (1994).

[45] A. L. Onishchik, Lectures on Real Semisimple Lie Algebras and Their Representations. Lectures in Mathematics and Phisics. European Mathematical Society, (2004).

[46] S. Ramanan, Orthogonal and spin bundles over hyperelliptic curves, Proc. Indian Acad. Sci. Math. Sci. 90 (1981), no.2, 151-166.

[47] A. Ramanathan, Stable principal bundles on a compact Riemann surface, Math. Ann. 213 (1975), 129-152.

[48] A. Ramanathan, Moduli for principal bundles over algebraic curves: I and II, Proc. Indian Acad. Sci. Math. Sci. 106 (1996), 301-328 and 421-449.

[49] A. H. W. Schmitt, A universal construction for moduli spaces of decorated vector bundles over curves, Habilitation thesis, University of Essen, (2000).

[50] A. H. W. Schmitt, Geometric Invariant Theory and Decorated Principal Bundles, Zurich Lectures in Advanced Mathematics, European Mathematical Society, (2008).

[51] C. T. Simpson, Constructing variations of Hodge structure using Yang-Mills theory and applications to uniformization, J. Amer. Math. Soc. 1 (1988), 867-918.

[52] C. T. Simpson, Higgs bundles and local systems, Inst. Hautes Études Sci. Publ. Math. 75 (1992), 5-95.

[53] C. T. Simpson, Moduli of representations of the fundamental group of a smooth projective variety I, Inst. Hautes Études Sci. Publ. Math. 79 (1994), 47-129.

[54] C. T. Simpson, Moduli of representations of the fundamental group of a smooth projective variety II, Inst. Hautes Études Sci. Publ. Math. 80 (1994), 5-79.

[55] R. O. Wells, Jr., Differential analysis on complex manifolds, Third Edition, SpringerVerlag, New York (2008). 